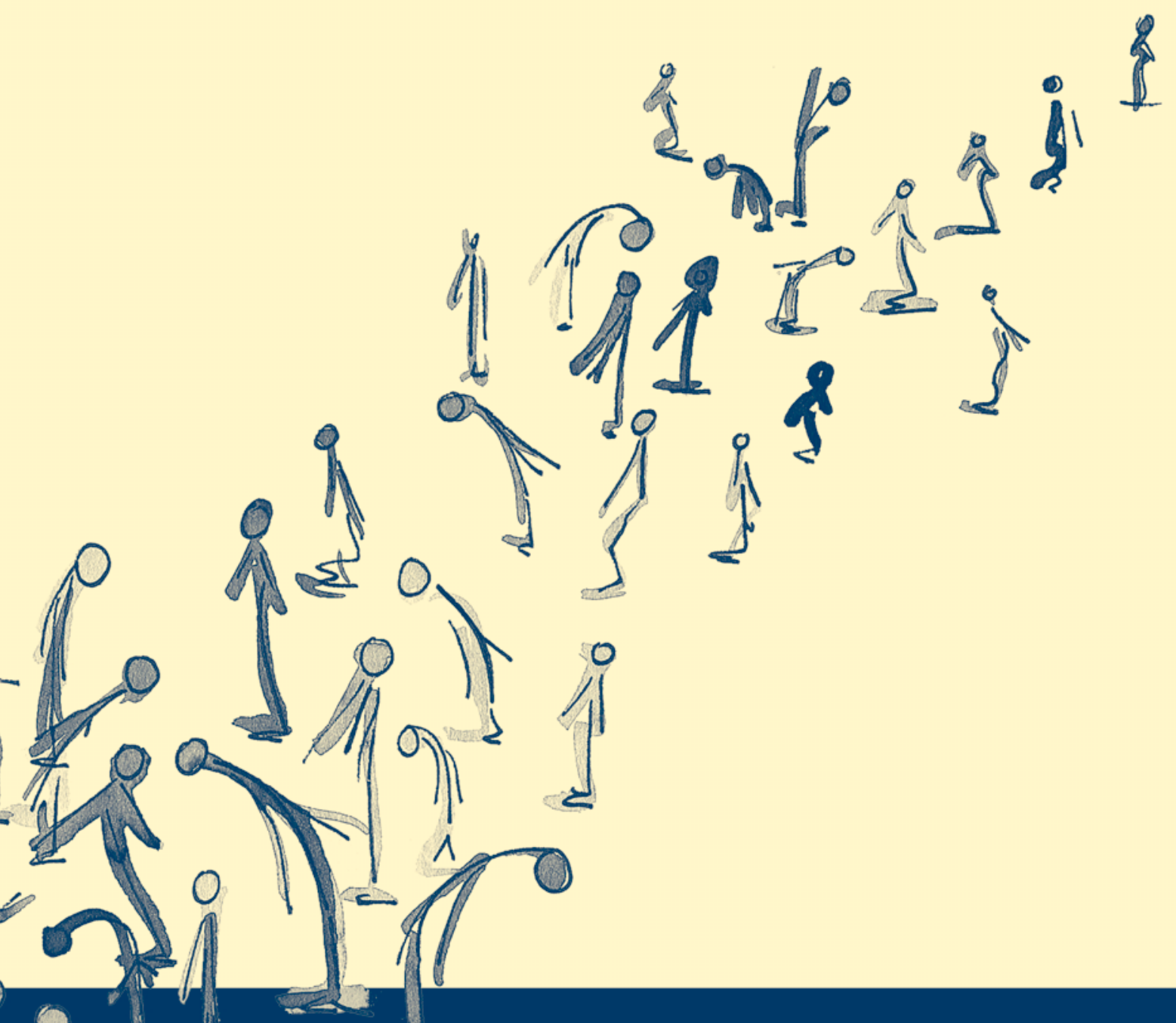

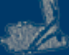

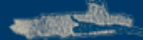

Psychomotor slowing and

planning deficits in schizophrenia

Bea Jogems-Kosterman 


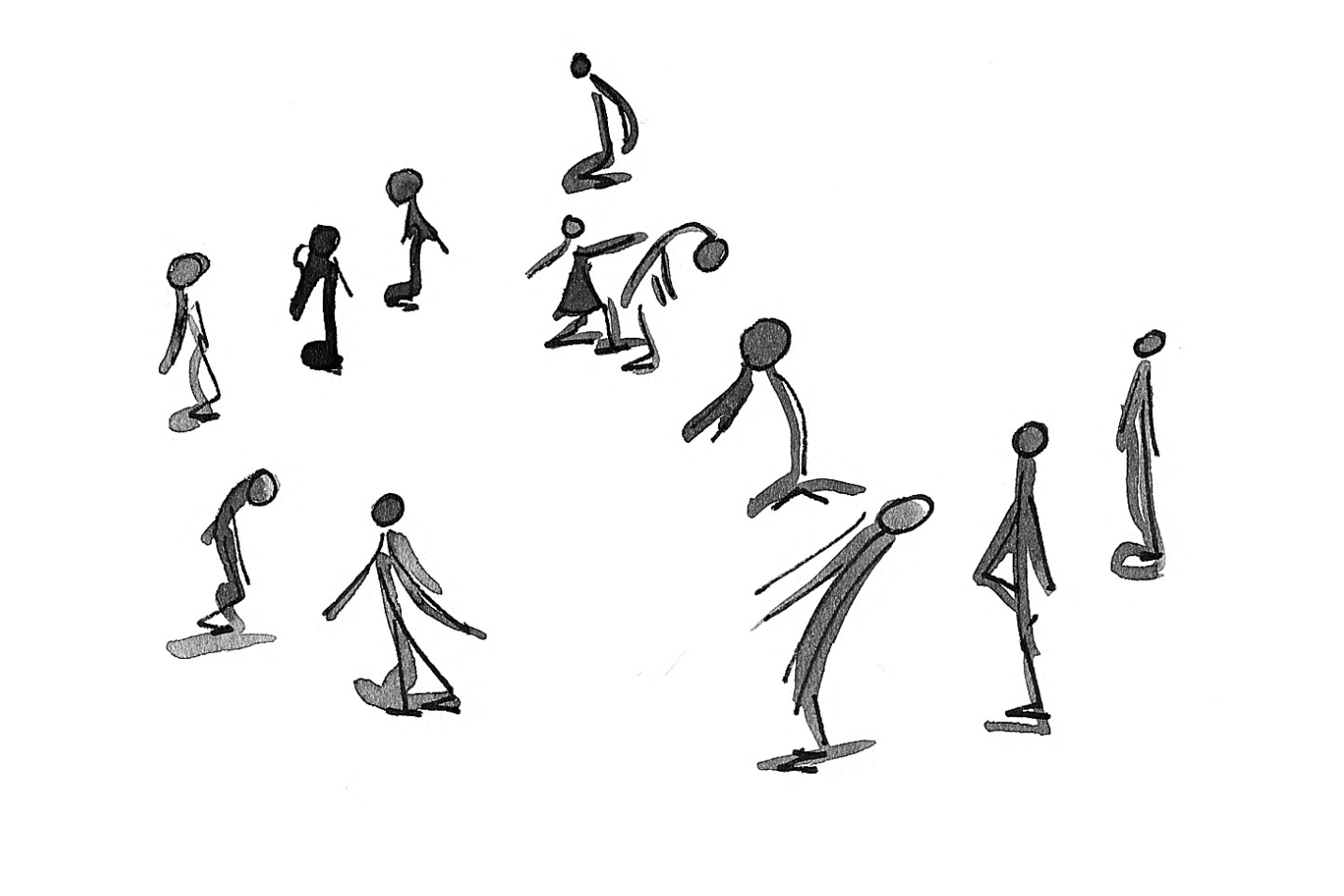





\section{Psychomotor slowing and planning deficits in schizophrenia}

Bea Jogems-Kosterman 



\title{
Psychomotor slowing and planning deficits in schizophrenia
}

\author{
Een wetenschappelijke proeve op het gebied van \\ de Sociale Wetenschappen
}

PROEFSCHRIFT

\author{
ter verkrijging van de graad van doctor \\ aan de Katholieke Universiteit Nijmegen, \\ op gezag van de Rector Magnificus Prof. dr. C.W.P.M. Blom, \\ volgens besluit van het College van Decanen \\ in het openbaar te verdedigen op \\ donderdag 1 juli 2004 \\ des namiddags om 1.30 uur precies \\ door \\ Bernardina Johanna Maria Jogems-Kosterman \\ geboren op 30 december 1969 te Wijk bij Duurstede
}




\section{Promotoren:}

Prof. dr. W. Hulstijn

Prof. dr. A.J.W.M. Thomassen

\section{Copromotor:}

Dr. J.J.M. van Hoof

\section{Manuscriptcommissie:}

Prof. dr. G.P. van Galen (voorzitter)

Prof. dr. J. van Os (Universiteit Maastricht)

Prof. dr. B.G.C. Sabbe (Universiteit Antwerpen)

\section{CIP - GEGEVENS KONINKLIJKE BIBLIOTHEEK DEN HAAG}

Jogems-Kosterman, Bernardina Johanna Maria

Psychomotor slowing and planning deficits in schizophrenia / Bernardina Johanna Maria JogemsKosterman - Thesis Katholieke Universiteit Nijmegen - with references - with a summary in Dutch and English

ISBN 90-9018094-X

Key words: schizophrenia, psychomotor slowing, planning, figure copying, movement analysis

Cover illustration: Esther Smits, 's-Hertogenbosch

Layout: Bea Jogems-Kosterman and John Wiering, Nijmegen

Printed by: Drukkerij van Gerwen, 's-Hertogenbosch

\section{Acknowledgements}

The printing of this thesis was financially supported by GGZ Oost Brabant, AstraZeneca, JanssenCilag, Organon Nederland and Eli Lilly Nederland. The research presented in this thesis was conducted in close collaboration with the NICI (Nijmeegs Institute for Cognition and Information). 
Voor mijn ouders

Voor Frank 



\section{Contents}

\section{Chapter 1}

General introduction

\section{Chapter 2}

Differentiation of cognitive and motor slowing in the Digit Symbol Test (DST):

Differences between depression and schizophrenia

\section{Chapter 3}

Antipsychotics, movement disorders and fine motor performance:

New device for the measurement of bradykinesia and tremor

\section{Chapter 4}

Psychomotor slowing and planning deficits in schizophrenia

\section{Chapter 5}

Is the planning of action more impaired in schizophrenic patients than in depressed patients? The effects of conflicting graphic production rules

\section{Chapter 6}

Planning in graphic production in patients with schizophrenia:

Conflicting results?

\section{Chapter 7}

Movement planning deficits in schizophrenia:

Failure to inhibit automatic response tendencies

\section{Chapter 8}

General discussion

Summary

Samenvatting

Curriculum Vitae

Publications

Dankwoord 



\title{
Chapter 1
}

\section{General introduction}

\begin{abstract}
Although delusions and hallucinations are perhaps the most intriguing and fascinating symptoms of schizophrenia, the illness is above all most obviously expressed in the behavior of patients and particularly in the way they perform motor actions. Schizophrenia is clearly associated with psychomotor deficits, in which the term 'psychomotor' refers to a wide range of human activities requiring both cognitive and (sensori)motor processes. How these psychomotor deficits can be observed in clinical practice is illustrated by the following example.

One of the patients on the ward is sitting motionless in a chair, without showing any emotional expression, and he is not able to either start an activity spontaneously or engage in it without being explicitly asked. Another patient, sitting next to him, is hardly moving except to brush his long hair away from his forehead in a fitful, repetitive motion. A third patient moves across the room, occasionally grasping objects from the table, but there seems no clear intention underlying his behavior.
\end{abstract}

Notwithstanding their large differences in appearance, these behavioral abnormalities all represent characteristic features of schizophrenia and may even co-exist in one and the same patient. A lack of initiative and activity is seen in many of the patients and constitute the core of the negative symptoms of schizophrenia (Crow, 1980) or the 'psychomotor poverty' syndrome (Liddle, 1987). The main components are a diminished and slowed activity in speech, emotion and movement. Clinical observation indicates that these symptoms are persistent and, therefore, typical for chronic schizophrenia (Liddle, 1993). Also illustrative for chronic schizophrenia is stereotypical behavior, which refers to the tendency to repeat an action over and over again, without any purpose (Frith, 1992). An impaired ability to select between activities characterizes the behavior of patients with disorganized behavior, for which Liddle (1987) used the term 'disorganization syndrome'. In many instances, these so-called 'psychomotor disorders' (Liddle, 1993) are not only lasting features but also seriously affect the life of the patient, and impose a heavy burden on those caring for them.

Obviously, because of disturbances in the cognitive and (sensori)motor processes underlying normal behavior, patients can be confronted with a kaleidoscope of problems in their daily functioning. To illustrate the relevance of the topic: over the last 15 years more than 1.000 publications on schizophrenia refer to the term 'psychomotor' in one way or the other (as identified in the MEDLINE computer database with the search terms 'schizophrenia and psychomotor'). ${ }^{1}$

\footnotetext{
${ }^{1}$ As a comparison: another very important topic like 'schizophrenia and memory' has resulted in about 1.500 scientific papers in the same period.
} 
Several investigators have attempted to understand the nature of these disorders. According to King (1991), patients with schizophrenia show psychomotor slowing because of a fundamentally impaired integration of sensory and motor processes, which, in addition, hinders the timing, sequencing and acquisition of more complex skills. Others, however, seem to argue in the opposite direction and advocate the idea that psychomotor disturbances are merely the overt expression of underlying cognitive deficits. For example, a patient who sits motionless in a chair might in fact experience an internal state of purposelessness. These authors (Widlöcher \& Hardy-Bayle, 1989; Frith, 1992; Liddle, 1993) put forward that the main problems of schizophrenia arise from failures in the generation, the planning or the monitoring of self-motivated behavior. In neuropsychology, the term 'executive dysfunction' is normally used for this type of deficits (Lezak, 1995).

Impairments in executive functions, including problems with working memory (for a recent review, see Palmer \& Heaton, 2000), and motor functions (for a recent review, see Boks et al., 2000) have both been reported widely in schizophrenia. Although these functions have traditionally been described as 'frontal functions', recent insight includes the growing recognition of the importance of cortical-subcortical circuits (Evarts et al., 1984; Cummings, 1993; Pantelis et al., 1997; Palmer \& Heaton, 2000). It is still questioned whether executive and motor deficits reflect distinct impairments occurring in different circuits, or a generalized impairment of most of these circuits (Poole et al., 1999). In classical neuropsychology, executive and motor functions are mostly measured by means of different neuropsychological tests (Lezak, 1995), despite the fact that they are hard to separate not only in daily life, but also in the test of consideration. Like most other neuropsychological tests, tests for executive functions draw on the specific cognitive function of interest (such as planning ability) in addition to more basic sensorimotor processes (Palmer \& Heaton, 2000). Ironically, Lezak (1995) must have felt this too, as executive functions and motor performances are described successively in the same chapter of her widely applied handbook 'Neuropsychological assessment'.

Consider once again one of the examples given above: the patient that is sitting motionless in his chair. There may be several reasons for his/her inability to generate action. Perhaps he/she is not able to identify a goal and/or develop subgoals, which suggests the involvement of higher cognitive, or executive, dysfunctioning. On the other hand, he/she might also be incapable to initiate the required motor action because of a basic problem with (sensori)motor processing. By means of classical neuropsychological research methods, it remains difficult to identify the process that contributes most to the impaired test performance. Therefore, in the present thesis a cognitive neuropsychological approach (e.g., Parkin, 1996) is applied to examine cognitive and (sensori)motor processes underlying psychomotor deficits in schizophrenia. Specifically, the aims of the present thesis are to investigate to what extent impairments in performing motor actions in schizophrenia (1) are due to psychomotor slowing, and/or (2) are affected by a more specific planning deficit. Another important question that we try to answer here is whether and how these impairments relate to clinical symptoms of schizophrenia.

Before describing the more specific research questions, an exposition will be given of the main clinical features of schizophrenia, of the cognitive and motor deficits associated with the 
illness, and, finally, the advances of a cognitive neuropsychological approach and the application of graphic tasks to study psychomotor deficits.

\section{Main clinical features of schizophrenia}

Schizophrenia is a complex mental disease, mostly characterized by acute phases of hallucinations, delusions and thought disorders (the so-called positive symptoms), and, on the long term, by apathy, flat affect and social withdrawal (the so-called negative symptoms). The current diagnosis of schizophrenia (DSM IV; American Psychiatric Association, 1994) requires the presence of two or more of the following symptoms: delusions, hallucinations, disorganized speech, severely disorganized behavior or catatonia, and negative symptoms. Only one of these symptoms is required when delusions are bizarre or hallucinations consist of one or more voices continuously commenting on the patient or conversing with each other. From the onset of symptoms, the patient must experience a breakdown of functioning in daily life (such as work, interpersonal relationships and self-care). Schizophrenia is a chronic condition, as the acute phase must be preceded by a period of at least six months in which signs of the disturbance are continuously present. This prodromal period may be characterized by only negative symptoms or two or more other symptoms in an attenuated form (e.g., odd beliefs and unusual experiences).

In the acute phase of the disease, antipsychotic medication is usually administered to the patient to reduce psychotic symptoms. If given successfully, the treatment will be continued in order to prevent relapses. An important disadvantage of the use of these antipsychotic drugs, however, is that they may induce unwanted motor side effects or extrapyramidal symptoms like bradykinesia, tremor, rigidity and akathisia (Ayd, 1961, 1983). In addition, it is generally assumed that about $20-30 \%$ of the patients do not profit from antipsychotic medication and remain psychotic with persistent hallucinations and/or delusions or disorganization of speech and behavior (e.g., Johnstone, 1991). About half of the patients with schizophrenia suffer from negative symptoms, such as a loss of initiative, apathy, psychomotor poverty and flattened affect (Cutting, 1995). These symptoms have a persistent character and are difficult to treat, and are associated with a loss of cognitive skills necessary for social functioning. Unfortunately, it has also proven extremely difficult to treat patients with disorganized speech and behavior successfully. For these patients, a setting that offers an extensive form of treatment, support and structure is often indicated (Withaar \& Arends, 2002).

Schizophrenia is viewed as one of the worst diseases affecting mankind and constitutes a severe public health issue (see anonymous contribution in Nature, 1988). The lifetime risk to develop schizophrenia is about 0.7 to $1 \%$ (Bromet \& Fennig, 1999). The clinical symptoms are usually not seen until adolescence. The neurodevelopmental hypothesis (Murray \& Lewis, 1987; Weinberger, 1987; see also Van Hoof, 2002), however, states that there is an abnormal development of the brain long before the apparent onset of the illness. It is argued that schizophrenia begins with abnormalities of neuronal migration and synaptogenesis during prenatal and perinatal life, resulting in misconnections between brain areas (Bloom, 1993; Crow et al., 1989). A number of biological and psychological risk factors are associated with the development of schizophrenia (for a review, see Bromet \& Fennig, 1999). The fact that symptoms arise so much later might be because brain 
areas responsible for these symptoms do not become mature and fully functional until adolescence (Weinberger, 1987). Yet, already during childhood, individuals with schizophrenia may display abnormalities of language (Jones et al., 1994), behavior (Done et al., 1994), and motor development (Walker, Savoie \& Davis, 1994).

In general, schizophrenia is associated with poor clinical and functional outcome. About one quarter of the patients show a benign course after their first psychotic episode, half of the patients recover after one of more relapses, whereas the remaining quarter display a chronic course with often requiring long-term hospitalization (for review studies, see Ram et al., 1992; Hegarty et al., 1994). From the patients with schizophrenia, the greater majority remains unmarried and unemployed (Bromet et al., 1996). In this perspective, cognitive deficits appear to restrict patients in terms of social and vocational functioning more than the severity of clinical symptoms do (Green, 1996; Green et al., 2000).

\section{Cognition in schizophrenia}

Potential cognitive impairments have been recognized since the early descriptions of schizophrenia by Emil Kraepelin (1855-1926) and Eugen Bleuler (1857-1939). Kraepelin (1899, 1919) used the term 'dementia praecox' (analogue to the expression 'démence précoce' introduced by the Benedict Augustin Morel, 1850) to describe a progressively developing psychic disease in young patients. Kraepelin noted that while certain basic psychic functions, such as perception, memory and orientation, may stay intact, these patients usually suffer from marked deficits in 'higher intellectual abilities' (Kraepelin, 1919). With this description, Kraepelin did not refer to impairments of the intellect in the strict sense (as measured by current IQ tests), but rather to volitional disorders or, what is now called, executive dysfunctions (see also Zec, 1995). This is well expressed in the following quotation:

... judgement is lost, the critical faculty, the creative gift, especially the capacity to make a higher use of knowledge and ability... The patients may also exhibit volitional activity of the greatest strength and endurance, but they are wholly incapable of ... carrying out a well-considered plan. (Kraepelin, 1919)

Eugen Bleuler $(1911,1950)$ introduced the term 'schizophrenia' (from the Greek and meaning literally "a mind torn asunder"). With this name, he focused on the major characteristic of the syndrome, the fragmentation of the brain functions that give rise to cognition, feelings and behavior. He preferred the term schizophrenia above dementia praecox, because he acknowledged (and by that time also Kraepelin) that the disease does not always start at younger ages and does not necessarily end in mental decline (dementia). Bleuler $(1916,1950)$ defined schizophrenia with his four well known A's: a loosening of Associations (disorganized thought), Affective disorders (diminished or inappropriate emotional responses), Ambivalence (an apparent inability to make decisions) and Autism (a loss of awareness of the external world and a preoccupation with the self and one's own thought). 


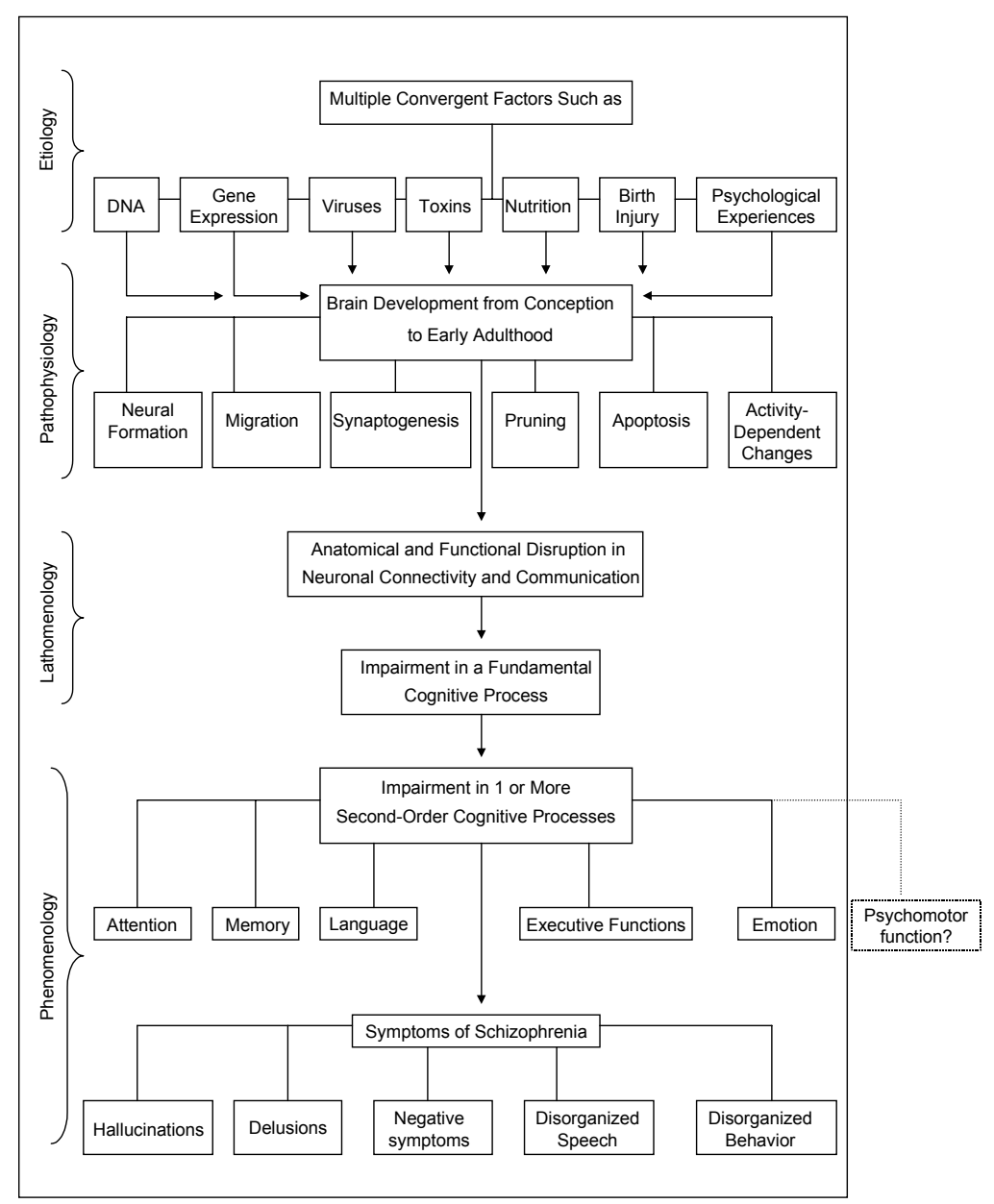

Figure 1: A general model of the development of schizophrenia (redrawn from Andreasen, 1999). The box with 'psychomotor function?' positioned outside the model was added by us.

In a recent attempt to define schizophrenia, Andreasen (Andreasen, 1999; Andreasen et al., 1999) proposed a neo-Bleulerian model to emphasize the illness' characteristic fundamental misconnection in thoughts and actions, for which she uses the term 'cognitive dysmetria'. According to Andreasen, this cognitive dysmetria constitutes a basic cognitive disturbance, which may lead to the diversity of symptoms and cognitive and emotional deficits in schizophrenia. She portrays the development of schizophrenia by means of an hourglass-shaped model (see Figure 1). There are multiple etiological factors at the "entry level" and a scale of symptoms at the "output level", but in the middle it is assumed that there is a fundamental cognitive malfunctioning that unifies the concepts of the illness. At the "output level", schizophrenia may not only give rise to a 
wide range of symptoms, but also to a widespread impairment on several cognitive domains. Remarkably, impaired psychomotor performance is not mentioned separately by Andreasen, but seems to be treated by her as the overt result of problems in one or more of the other domains, in particular executive functioning. Notwithstanding this less prominent role of psychomotor deficits per se, Andreasen assumes that the fundamental disconnection occurs in the same circuit (corticocerebellar-thalamic-cortical circuit) that permits individuals to perform the very rapid adjustments that are required for complex motor acts. Impairment in this circuit not only results in various types of motor dysmetria, such as poor tandem gait or past-pointing, but may also lead to a poor coordination of thoughts and actions. According to Andreasen et al. (1999) schizophrenia may therefore be conceptualized as a disease that is characterized by a basic disturbance in the "smooth planning and execution of both motor and cognitive activities (p. 916)". Unfortunately, as the model of Andreasen primarily is a cognitive model, psychomotor performance does not get equal attention when compared to the other neuropsychological domains (see our hypothetical positioning of the psychomotor domain in Figure 1). In addition to the model of Andreasen, there are other competitive neurocognitive models for schizophrenia, e.g., Goldman-Rakic (1994; the inability to guide behavior by representations), Braff (1993; abnormalities in information processing), Frith (1992; the inability to think in metarepresentations), Van Hoof (2002, 2003; deficient drive and guidance mechanisms). Which of these models explains most appropriately the fundamental deficit(s) of schizophrenia will most likely require many years of research.

\section{Executive deficits}

As exemplified before, Kraepelin's (1919) descriptions of problems of patients with schizophrenia bear striking similarity to what we now consider deficits of executive function: the inability to engage in complex goal-directed behavior, to anticipate outcomes, and to adjust attention to changing environmental demands. Executive functions include the following four components: (1) the ability to formulate new activities or plans spontaneously, (2) the capability to conceptualize change for the present, make choices and anticipate consequences, and develop a structure to achieve the goal, (3) the ability to carry out goal-directed behavior which requires the ability to initiate, maintain, switch and stop sequences of complex behavior, and (4) the ability to monitor effective performance (Lezak, 1995). A difficulty with the evaluation of these executive functions is that, although these deficits have an overwhelming impact on daily life functioning, they are hard to catch by standard psychometric methods (a paradox described by Luria (1980)). None of these tests is a pure measure of executive functioning; instead, they draw upon a range of cognitive abilities (as well as more basic sensorimotor skills), some more salient than others. In addition, most of these tests involve complex tasks and it is often difficult to determine which task element contributes most to impaired test performance.

The most widely used tests for executive functions in schizophrenia (for a review, see Palmer \& Heaton, 2000) are the Wisconsin Card Sorting Test (WCST) that assesses the ability to shift strategies efficiently in response to environmental feedback; the Stroop Color Word Interference Test measuring the capacity to inhibit an automatic response; Tower tasks requiring the planning ahead of a sequence of movements so that the positions of a number of beads or disks 
(varying in either color or size) correspond to a goal position; Verbal Fluency Tests assessing the ability to freely generate words from a certain category (e.g., animals); the Trail Making Test (part B) requiring the ability to connect numbers and letters by alternating between the two sequences (1A-2-B etc.); Maze tests requiring the planning of an optimal route.

Impairments in executive functions are commonly reported among patients with schizophrenia, but they are also an important dimension on which patients vary widely (Palmer \& Heaton, 2000). Executive dysfunctions are more often reported in patients with persistent negative symptoms (or 'psychomotor poverty') and/or cognitive disorganization than in patients with predominantly psychotic symptoms. Negative symptoms have particularly been found associated with impaired generation and planning abilities (Liddle \& Morris, 1991, Berman, et al., 1997; Brazo et al., 2002). Cognitive disorganization mainly involves impairments in the ability to inhibit automatic responses (Liddle \& Morris, 1991; Baxter \& Liddle, 1998; Mahurin et al., 1998; Brazo et al., 2002).

The 'Willed action' Model of Frith (Frith, 1987; Frith \& Done, 1988; Frith, 1992; Cahill \& Frith, 1996) deserves special attention in the context of executive dysfunctions. This model details how the behavioral symptoms of schizophrenia may reflect dysfunctions in a general action system. Central to the model of Frith are the two routes to action: the stimulus-driven route (i.e. automatic) and the willed route (i.e. self-initiated). In the model it is assumed that patients with schizophrenia have particularly difficulty with the willed route, i.e. with the production of self-initiated or willed actions, while the stimulus-driven route remains largely intact. Patients with negative symptoms are supposed to show poverty of action because they have difficulty to generate and plan self-motivated actions in the absence of external cues. However, according to Frith et al. problems with willed actions can also lead to disorganized or stereotyped behavior. In such cases the patient is unable to generate the appropriate behavior of his/her own will, and also fails to suppress inappropriate behavior. Recent actions are repeated (i.e., perseverations) or responses are made to irrelevant stimuli, so that action plans cannot be carried through to completion. This is supposed to lead to disorganized speech and behavior (Frith, 1992).

Despite the potential importance of executive dysfunctions for the clinical picture of schizophrenia and related problems in social and occupational functioning (Green, 1996; Green et al., 2000), numerous studies have shown that schizophrenia is not only associated with limited executive skills, but also with impairments in more basic psychomotor measures (Braff et al., 1991; Goldstein, Beers \& Shemansky, 1996; Pantelis et al., 1997; Bilder et al., 2000). Bilder et al. (2000), for example, found that motor deficits (assessed by means of tests for tapping, dexterity and visualspatial ability) were clearly impaired in a group of nearly 100 patients with schizophrenia and that these remained present even after controlling for other cognitive functions, such as executive function and memory. By means of a computerized version of the Tower of London, Pantelis et al. (1997) demonstrated that patients with schizophrenia not only made fewer perfect solutions and required more moves to completion, but also needed more 'pure' movement time to replace the beads which suggests impairment in the (sensori)motor requirements of the task. In addition, Poole et al. (1999) showed that patients who have both executive and motor deficits had the least favorable clinical, vocational and social outcome. 


\section{Psychomotor slowing and planning deficits}

Psychomotor deficits should not be restricted to problems with executive functioning. Although schizophrenia is not usually considered to be a "motor disease" (most handbooks on schizophrenia do not even dedicate a chapter to this topic), many indicators of motor dysfunction are present. Observations of motor impairments in patients with schizophrenia date back from Bleuler (1908) and Kraepelin (1919). These observations included irregular timing and spacing of steps during walking as well as reduced efficiency and coordination of fine movements during handiwork and crafts.

Outspread fingers often show fine motor tremor... The expression of the face, vacant, immobile, like a mask, astonished, is sometimes reminiscent of the rigid smile of the Aeginetans.... Simple movements are stiff, slow, forced. (Kraepelin, 1919)

Interest in motor deficits in schizophrenia has shown a revival over the past decades as studies have demonstrated their occurrence even among neuroleptic-naive patients (Owens, Johnstone \& Frith, 1982; Caliguiry, Lohr \& Jeste, 1993; Caliguiry \& Lohr, 1994). Before that time, the notion that motor deficits are primary features of schizophrenia has been obscured by the fact that they were not only seen as secondary to cognitive and emotional deficits (Bleuler, 1950), but also as side effects of antipsychotic medication (Ayd, 1961, 1983). However, this is not justified, as, for example, slowed reaction time is one of the oldest and most robust findings that differentiate patients with schizophrenia from healthy control subjects (Huston, Shakow \& Riggs, 1936).

Movement disorders in schizophrenia have also been frequently assessed by means of neurological examination. A review of Boks et al. (2000) confirmed that the prevalence of most of the 30 neurological soft signs for movement disorders is higher in patients with schizophrenia than in normal controls, but there were fewer differences found between schizophrenia and mooddisorder patients. Impaired motor coordination (dysdiadochokinesia, tandem walk, finger-thumb opposition, and finger-to-nose) appeared to be more specific to schizophrenia than to mooddisorder. Further, signs of impaired motor sequencing were also more prevalent in schizophrenia when compared to healthy controls, but these have unfortunately not been studied in mood-disorder patients.

Although motor deficits are commonly observed in schizophrenia, the exact nature of these motor disturbances is still far from clear. Some studies suggest that the problem lies primarily in the planning phase of the movement and not in its execution (Malla et al., 1995; Carnahan et al., 1997). Delevoye-Turrell et al. (2003) showed that it was the sequencing of the movement more than the anticipatory ability that was most impaired in a group of patients. In addition, the study of Caliguiry et al. (1993) confirmed that neuroleptic-naive patients might even show tremor and rigidity in the execution of a simple movement. In this context it is important to notice that problems with motor control can be expressed in several ways. Even the planning and execution of an apparently simple motor action requires several processes. Willingham (1998) distinguishes four processes: a strategic or higher-level action planning process that identifies the goal of the movement (which was previously mentioned as an executive function), perceptual motor integration necessary to select the 
target for movement (cf. King, 1991), the planning of the required sequence of movements (see Delevoye-Turrell, Giersch \& Danion, 2003), and, finally, a dynamic process that is responsible for the desired pattern of muscle activity (see Caliguiry, Lohr \& Jeste, 1993).

Apparently, simple motor actions are far more complex than most clinicians seem to imply when they mention motor deficits in patients with schizophrenia. This can be illustrated by describing the processes that are involved when, for example, grasping a cup of coffee for drinking. Prior to this action, a need for drinking the coffee is likely (otherwise: why should one perform this action?). This process can be seen as the goal selection part (1). Also prior to the action, perceptual processes are necessary for the visual inspection of the cup in order to establish its relevant features like shape, size and relative distance from the body. In addition, visual-spatial processes are needed to determine and plan the endpoint of the movements and, for example, the extent to which the hand needs to be opened when approaching the cup. These processes can be denoted by the term perceptual motor integration (2). Next, the right movement sequence must be planned, which means that the cup has first to be grasped before it can be brought to the mouth: sequence planning (3). Finally, there is the initiation and execution of the muscle activity (4) necessary for the required movement(s) as well as the monitoring of visual feedback to correct errors (c.f. Willingham, 1998).

Although the framework of motor control as proposed by Willingham (1998) might suggest that these four processes take place in exactly this order, the planning and execution of movements can better be viewed as a dynamic process in which several processes are available in a conscious or effortful manner at any time. According to Willingham, most of these processes take place even outside of awareness, particularly when one performs an over-learned action. Going back to our example of reaching for a cup of coffee: one is probably aware of wanting to drink the coffee but unaware of selecting the targets of movements, sequencing them and provoking the right muscle forces. These processes will usually only be transformed to the conscious mode when one makes an error or believes that the initiated action would fail.

What does this help us in our understanding of psychomotor slowing and planning deficits in schizophrenia? Most important is that we have to acknowledge that these terms strongly depend on the level of investigation. At least three different levels can be distinguished: clinical psychiatry, neuropsychology and human movement science.

Psychomotor slowing, when observed in clinical psychiatry, entails problems not only with motor activities, but also with cognitive, emotional, and even social behavior. It includes, for example, a diminished spontaneity of gestures and speech, loss of interest, apathy, decreased performances at school, work and home, and social withdrawal. These impairments are not unique for schizophrenia, but can also be observed in several other psychiatric and neurological diseases, like depression, Parkinson's disease, frontal lobe damage and subcoritical dementia (Benson, 1990; Bermanzohn \& Siris, 1992). In particular, the question whether schizophrenia and depression can be characterized by a similar type of slowing is of theoretical importance (Widlöcher \& Hardy-Bayle, 1989; Frith, 1992); moreover it has also clinical relevance. From the theoretical point of view, it is argued that the causes of psychomotor slowing in schizophrenia and depression are not the same. In schizophrenia it is assumed that psychomotor slowing is mainly the result of problems with generating self-motivated actions, whereas in depression it arises particularly due to a lack of 


\section{0 | Chapter 1}

energy to fulfill these actions (Widlöcher \& Hardy-Bayle, 1989; Frith, 1992). In clinical practice, however, it is known that in schizophrenia slowing can also arise due to a concomitant depression. Brébion et al. (2000), for example, found evidence of a depressive-like slowing in a group of patients with schizophrenia. To address this issue, we included depressed patients in some of the studies that are part of this thesis and compared their performances with those of patients with schizophrenia. At the same psychiatric level, planning deficits become most obvious when a patient is not able to arrange a series of daily activities in an orderly way, which may lead to a poverty of action, stereotyped or even chaotic behavior. This broad psychiatric concept of clinically observable impairments illustrates how difficult it is to understand the nature of problems patients are faced with in daily life. Liddle (1993) even used the term 'psychomotor disorders' to cover the wide range of behavioral deficits associated with schizophrenia.

A more restricted use of the term 'psychomotor' can be found in neuropsychology. In the following chapters of the present thesis, the term 'psychomotor slowing' refers in particular to the type of slowing that can be observed at the neuropsychological level: an essential slowing of both cognitive and motor processes. In addition to this, neuropsychology tries to study planning as well as motor deficits by means of distinct tests that focus on a certain activity. However, as we concluded earlier, most planning tests also require more basic (sensori)motor processes. Conversely, we now acknowledge that most motor actions also demand planning ability (e.g., goal selection and/or sequencing). For example, some of the type of tasks that are mentioned by King (1991) as being basic psychomotor tests, i.e. tapping tasks and dexterity tasks, do not solely challenge perceptual motor integration but also other processes, in particular sequencing. Tapping tasks, for example, require the same sequence to be selected repetitively. Pegboard tests for manual dexterity require a sequence of fast and correct insertions of pegs into small holes. In one variant, i.e. the Grooved Pegboard, pegs have to be rotated first, which requires in addition the planning and execution of efficient movements. It is this type of planning ability on which investigators in human motor control focus their research work.

At the third level, that of human movement science, the most restricted approach to psychomotor slowing can be found. From this perspective, psychomotor slowing can be even observed in one single action, like reaching for a cup of coffee. Although there are of course several other theories on motor control, for describing the goals of the studies in the present thesis and the applied tasks, the framework of Willingham (1998) seems suitable. It provides a detailed (cognitive) neuropsychological account of motor deficits in neurological and psychiatric patients, and the processes involved. For the sake of convenience, I will use the terms sensori(motor) and basic psychomotor in this thesis when tasks tap the processes of 'perceptual-motor integration' and/or 'dynamic selection of muscle activity' in particular. The term planning, in addition, will be applied when tasks make a greater appeal to 'goal selection' and/or 'sequencing'. In a sense, this way of putting weights on one or more processes dependent on the type of task, prevents us from using the more simple dichotomy of cognitive processes necessary for the planning or preparation of the movements (mostly captured by 'reaction time') and motor processes needed for the execution of the movement (as measures by 'movement time'). As we could see, motor actions can better be viewed as involving both planning and basic psychomotor processes simultaneously. 


\section{Cognitive neuropsychology}

It was made clear above that the classical neuropsychological approach is less appropriate for the examination of cognitive and (sensori)motor deficits in schizophrenia. These tests were initially developed for patients with circumscribed brain damages, but they are too 'crude' and fall short of explaining the complex and diverse deficits in schizophrenia (Frith, 1995, 1999). Therefore, in the studies of the present thesis tasks were developed from a cognitive neuropsychological viewpoint (e.g., Parkin, 1996). In these tasks it is not one output measure that counts, but rather the result of the manipulation of different experimental variables within the task. These variables are manipulated in such a way that as much as possible they affect a single process, like the planning of a sequence of movements. When such an experimental variable has a greater impact in patients than in control subjects, this indicates that patients are likely to experience more problems in the cognitive process that is influenced by this variable. A fruitful application of this approach can be conceived in the use of graphic tasks.

\section{Graphic tasks}

Graphic tasks, such as writing and drawing, offer excellent opportunities to study psychomotor deficits in schizophrenia from the cognitive neuropsychological viewpoint. The diagnostic value of graphic tasks has been acknowledged from the beginning of test psychology, as they are elements of more than 20 neuropsychological tests. Writing and particularly drawing tasks are usually applied to measure sensorimotor processes and visual-spatial skills (see Van Mier, 1992, for a brief overview of these tests). In addition to these explicit graphic tasks, there are several other neuropsychological tests that implicitly require the production of drawing or writing movements, such as the Digit Symbol Test (DST) or the Trail Making Test (TMT). However, apart from measuring drawing or writing ability in the strict sense, these tests tap other processes like working memory, attention and cognitive flexibility.

The evaluation of handwriting performance can be very helpful in understanding neurological writing disorders like apraxic agraphia, dysgraphia, micrographia or writer's cramp. In the case of studies investigating other movement disorders, such as in schizophrenia, analyses of drawing movements in tasks consisting of copying simple and/or more complex figures (e.g., lines, L-shaped patterns, letters and abstract patterns) are, however, also of great value. The advantages of assessing writing and drawing skills over typing or keyboarding are that they are mastered at an earlier age and by a larger proportion of the population. Moreover, if these movements are recorded by means of a digitizer (graphic tablet) and a special electronic pen and analyzed using advanced software (like OASIS, De Jong et al., 1996), the combination of precise measurement of each detail of the movement and the amount of experimental manipulation that such studies allow offer a major opportunity for cognitive neuropsychology (see also Hulstijn, 1996). It has already proven to be of great value for the study of psychomotor slowing in depression (Van Hoof et al., 1993; Sabbe et al., 1996a, 1996b). This method was also employed in the studies of the present thesis. 


\section{Outline of the thesis}

The first aim of this thesis is to investigate whether schizophrenia can be associated with a marked psychomotor slowing. To this end, we used simple fine (sensori)motor tasks that were performed on the digitizing tablet, allowing the separate analysis of several movement variables, such as the time needed to initiate a movement, and the time needed for execution. The second aim of the thesis is to search for more specific planning problems in schizophrenia when task requirements increase. For this purpose, we applied tasks in which we manipulated certain planning demands, such as the number of line elements and the familiarity of the stimuli to be copied, the spatial position of these in the working field, and the ease with which automatic preferences could be followed. The effects of these manipulations on task performance were studied in detail. The third goal of this thesis was to examine whether and how task performances relate to clinical symptomatology.

Chapter 2 of this thesis addresses a primary question, i.e. whether psychomotor slowing in a group of patients with schizophrenia differs from the slowing that can be observed in a group of depressed patients. It was investigated whether cognitive and motor processes contribute similarly or distinctly to performance on a standard neuropsychological test for general processing speed, the Digit Symbol Test (DST; Wechsler, 1956), in these patient groups.

Psychomotor slowing might resemble features of drug-induced parkinsonism in schizophrenia, particularly bradykinesia, which literally means 'slow movement'. Patients with bradykinesia usually show slowness of movement, difficulty with initiating movements, and a loss of automatic behavior, such as swinging of the arms while walking. The study described in Chapter 3 was, however, not designed to distinguish psychomotor slowness induced by the use of antipsychotic drugs from disease-related psychomotor slowing. Instead, it served as a first exploration of whether and how psychomotor performance relate to clinical features of bradykinesia in schizophrenia, as assessed by means of the Schedule of the Assessment of Drug Induced Movement Disorders (SADIMoD; Loonen et al., 2000, 2001).

What happens when the planning and organization demands increase? The study presented in Chapter 4 aimed at investigating the relative contribution of cognitive and motor processes to the slowing in schizophrenia by means of a simple line-drawing task, the DST, and a more complex figure-copying task. In this complex figure-copying task, the number of line elements and the familiarity of the patterns were systematically varied. The four 'ambitious' hypotheses that were tested were: (1) if it is true that schizophrenia is associated with basic psychomotor slowing (King, 1991), these patients should show a more or less generalized slowing, irrespective of the task complexity; (2) if cognitive slowing in schizophrenia is more pronounced than motor slowing (Nelson et al., 1990), patients with schizophrenia should be particularly retarded in those movement variables that reflect cognitive processing; (3) if planning of movements is disturbed in schizophrenia (Frith, 1992; Widlöcher \& Hardy-Bayle, 1989), the effects of increased cognitive task complexity should be greater in patients than in healthy controls; and (4) since psychomotor slowing is categorized as a negative symptom (Liddle, 1987), this slowness should be more pronounced in patients with clear negative symptoms.

Whereas Chapter 2 examines the differences between schizophrenia and depression in the cognitive versus motor load of processing speed, Chapter 5 attempts to highlight differences in 
planning ability. For this purpose we applied an 'elegant' task, in which movement planning, but not working memory capacity, was challenged. Impaired working memory revealed to be a confounding factor in the interpretation of the results of most planning tasks (e.g., Pantelis et al., 1997): because of a limited working memory capacity, a person might start the action before he has planned the required sequence of movements. This was also one of the factors that could have affected patients' performances in the study of Chapter 4. In the study reported in Chapter 5, we examined the question whether the ease of applying highly practiced and automatic preferences in graphic production (so-called graphic production rules) (Thomassen \& Tibosch, 1991; Thomassen, Meulenbroek \& Tibosch, 1991) affects the speed of copying simple patterns in patients with schizophrenia and depressed patients in a similar or in a differential way. Whereas for the copying of so-called 'non-conflicting' patterns (in which rules can be followed) a routine and automatic strategy suffices, copying 'conflicting' patterns (in which one or more rules have to be violated) requires the planning of the most efficient movement sequence and the anticipation of conflicts between rules. In our study, it was hypothesized that because of a lack of planning (Frith, 1992; Widlöcher \& Hardy-Bayle, 1989), patients with schizophrenia would show fewer differences between copying 'non-conflicting' patterns and 'conflicting' patterns when compared to depressed patients.

The patients with schizophrenia studied in Chapter 5 were all future outpatients: their psychiatrists judged them to be able to leave the psychiatric hospital (Fakkers et al., 2002). In order to replicate these findings, we extended our examination to a broader group of patients, in particular to the large population of hospitalized patients (Hulstijn et al., 2001). These more recent observations showed that the group of hospitalized patients performed more similar to the depression group (i.e., with greater differences between the 'non-conflicting' and 'conflicting' patterns) than to the schizophrenia outpatient group. The aim of the study described in Chapter 6 is to clarify these differences between patient groups. The questions to be answered were: (1) whether the distinction between inpatients and outpatients could be explained by differences in psychopathology and, particularly in depressive symptoms; (2) whether group differences emerge because some of the patients with schizophrenia show a kind of slowing similar to the kind that underlies the symptoms of a depression.

Difficulties with planning can be expressed in several ways. A subject might show a tendency to produce solutions before these are fully planned in advance. As mentioned before, one possible explanation for this is that the subject is not able to hold sufficient information 'on line' while planning a sequence of movements, with the result that he/she produces more errors, omissions and/or hesitations during the execution of the task. Indeed, in the study of Chapter 4 , the patients with schizophrenia did not show the expected prolongation of initiation time before copying the most complex figures, but they needed more reinspection time and pausing time during the copying phase. In addition, a subject might be less capable of planning the most efficient movement sequence, which was particularly the topic of Chapter 5 and 6.

The study presented in Chapter 7 explores two further important planning issues. First, the planning of movements can be more or less difficult dependent on the visual-spatial demands of a particular action. Therefore, in this study subjects were asked to copy simple two-line stimuli that 


\section{4 | Chapter 1}

varied with respect to their spatial position in a small work field. It was hypothesized that patients with schizophrenia would show longer initiation times in copying patterns that are located in different positions. Second, it has frequently been suggested that patients with schizophrenia may have planning difficulties due to an impaired ability to inhibit strongly cued automatic responses (Frith, 1992). To address this issue we applied a task in which the point from which the subject had to start copying was imposed (indicated by a black dot in the pattern itself). The imposed starting point either corresponded or contrasted with a highly preferred starting point. We hypothesized that forcing patients with schizophrenia to inhibit the application of a highly preferred rule would increase their initiation time more strongly than those of control subjects.

In the concluding Chapter 8 a critical review of the studies is provided and the implications for clinical practice and future research are discussed. 


\section{References}

American Psychiatric Association (1994). Diagnostic and statistical manual of mental disorders (4th edition). Washington, D.C.: APA.

Andreasen, N. (1999). A unitary model of schizophrenia. Bleuler's "fragmented phrene" as schizencephaly. Archives of General Psychiatry, 56, 781-787.

Andreasen, N.C., Nopoulos, P., O’Leary, D.S., Miller, D.D., Wassink, T., \& Flaum, M. (1999). Defining the phenotype of schizophrenia: Cognitive dysmetria and its neural mechanisms. Biology Psychiatry, 46, 908920.

Anonymous (1988). Where next with psychiatric illness? Nature, 336, 95-97.

Ayd, F.J. (1961). A survey of drug-induced extrapyramidal reactions. JAMA, 175, 1054-1060.

Ayd, F.J. (1983). Early-onset neuroleptic-induced extrapyramidal reactions: A second survey, 1961-1981. In: J.T. Coyle \& S.J. Enna (Eds.), Neuroleptics: Neurochemical, behavioral and clinical perspectives. New York, N.Y.: Raven Press.

Baxter, R.D., \& Liddle, P.F. (1998). Neuropsychological deficits associated without schizophrenic syndromes. Schizophrenia Research, 30, 239-249.

Benson, F. (1990) Psychomotor retardation. Neuropsychiatry, Neuropsychology and Behavioral Neurology, $3,36-47$.

Berman, I., Viegner, B., Merson, A., Allan, E., Pappas, D., \& Green, A.I. (1997). Differential relationships between positive and negative symptoms and neuropsychological deficits in schizophrenia. Schizophrenia Research, 25, 1-10.

Bermanzohn, P.C., \& Siris, S.G. (1992) Akinesia: A syndrome common to parkinsonism, retarded depression, and negative symptoms of schizophrenia. Comprehensive Psychiatry, 33, 221-232.

Berrios, G.E. (1985). Positive and negative symptoms and Jackson. A conceptual history. Archives of General Psychiatry, 42, 95-97.

Bilder, R.M., Goldman, R.S., Robinson, D., Reiter, G., Bell, L., Bates, J.A., Pappadopulos, E., Wilson, D.F., Alvir, J.M.J., Woerner, M.G., Geisler, S., Kane, J.M., \& Lieberman, J.A. (2000). Neuropsychology of first-episode schizophrenia: Initial characterization and clinical correlates. American Journal of Psychiatry, 157, 549-559.

Bleuler (1950). Dementia praecox or the group of schizophrenias (transl. [from the German] by Joseph Zinkin). New York, NY: International Universities Press

Bloom, F.E. (1993). Advancing a neurodevelopmental origin for schizophrenia. Archives of General Psychiatry, 50, 224-227.

Boks, M.P.M., Russo, S., Knegtering, R., \& Van den Bosch, R.J. (2000). The specificity of neurological signs in schizophrenia: A review. Schizophrenia Research, 43, 109-116.

Braff, D.L. (1993). Information processing and attention dysfunctions in schizophrenia. Schizophrenia Bulletin, 19, 233-259.

Braff, D.L., Heaton, R., Kuck, J., Cullum, M., Moranville, J., Grant, I., \& Zisook, S. (1991). The generalized pattern of neuropsychological deficits in outpatients with chronic schizophrenia with heterogeneous Wisconsin Card Sorting Test results. Archives of General Psychiatry, 48, 891-898. 


\section{6 | Chapter 1}

Brazo, P., Marié, R.M., Halbecq, I., Benali, K., Segard, L., Delamillieure, P., Langlois-Théry, S., Van Der Elst, A., Thibaut, F., Petit, M., \& Dollfus, S. (2002). Cognitive patterns in subtypes of schizophrenia. European Psychiatry, 17, 155-162.

Brébion, G., Amador, X., Smith, M., Malaspina, D., Sharif, Z., \& Gorman, J.M. (2000). Depression, psychomotor retardation, negative symptoms, and memory in schizophrenia. Neuropsychiatry, Neuropsychology and Behavioral Neurology, 13, 177 - 183.

Bromet, E.J., Jandorf, L., Fennig, S., Lavelle, J., Kovasznay, B., Ram, R., Tanenberg-Karant, M., \& Craig, T. (1996). The Suffolk County Mental Health Project: Demographic, pre-morbid and clinical correlates of 6-month outcome. Psychological Medicine, 26, 953-962.

Bromet, E., \& Fennig, S. (1999). Epidemiology and natural history of schizophrenia. Biological Psychiatry, $46,871-881$.

Cahill, C., \& Frith, C. (1996). A cognitive basis for the signs and symptoms of schizophrenia. In: C. Pantelis, H.E. Nelson \& T.R.E. Barnes (Eds.), Schizophrenia: A neuropsychological perspective (pp. 373-395). Chichester: John Wiley \& Sons.

Caliguiry, M.P., Lohr, J.B., \& Jeste, D.V. (1993). Parkinsonism in neuroleptic-naive schizophrenic patients. American Journal of Psychiatry, 150, 1343-1348.

Caliguiry, M.P., \& Lohr, J.B. (1994). A disturbance in the control of muscle force in neuroleptic-naive schizophrenic patients. Biological Psychiatry, 35, 104-111.

Carnahan, H., Aguilar, O., Malla, A., \& Norman, R. (1997). An investigation into movement planning and execution deficits in individuals with schizophrenia. Schizophrenia Research, 23, 213-221.

Crow, T.J. (1980). Molecular pathology of schizophrenia: More than one disease process? British Medical Journal, 280, 66-68.

Crow, T.J., Ball, J., \& Bloom, S.R. (1989). Schizophrenia as an anomaly of development of cerebral asymmetry: A postmortem study and a proposal concerning the genetic basis of the disease. Archives of General Psychiatry, 46, 1145-1150.

Cummings, J.L. (1993). Frontal-subcortical circuits and human behavior. Archives of Neurology, 50, 873880.

Cutting, J. (1995). Descriptive psychopathology. In: S.R. Hirsch \& D.R. Weinberger (Eds.), Schizophrenia (pp. 15-27). Oxford: Blackwell Science.

De Jong, W.P., Hulstijn, W., Jogems-Kosterman, B.J.M., \& Smits-Engelsman, B.C.M. (1996). OASIS software and its application in experimental handwriting research. In: M.L. Simner, C.G. Leedman \& A.J.W.M. Thomassen (Eds.), Handwriting and drawing research: Basic and applied issues (pp. 429440). Amsterdam: IOS Press.

Delevoye-Turrell, Y., Giersch, A., \& Danion, J.M. (2003). Abnormal sequencing of motor actions in patients with schizophrenia: Evidence from grip force adjustments during object manipulation. American Journal of Psychiatry, 160, 134-141.

Done, D.J., Crow, T.J., Johnstone, E.C., \& Sacker, A. (1994). Childhood antecedents of schizophrenia and affective illness: Social adjustment at ages 7 and 11. British Medical Journal, 309, 699-703.

Evarts, E.V., Kimura, M., Wurtz, R.H., \& Hikosaka, O. (1984). Behavioral correlates of activity in basal ganglia neurons. Trends in Neuroscience, 7, 447-453. 
Fakkers, G.P.F.M., Jogems-Kosterman, B.J.M., Loonen, A.J.M., Van Hoof, J.J.M., \& Hulstijn, W. (2002). Changes in clinical functioning and neurocognitive performances in patients with schizophrenia during the first years of deinstitutionalization. Schizophrenia Research, 53, 127.

Frith, C.D. (1987). The positive and negative symptoms of schizophrenia reflect impairments in the perception and initiation of action. Psychological Medicine, 17, 631-648.

Frith, C.D., \& Done, D.J. (1988). Towards a neuropsychology of schizophrenia. British Journal of Psychiatry, 153, 437-443.

Frith, C.D. (1992). The Cognitive neuropsychology of schizophrenia. Hove: Lawrence Erlbaum Associates.

Frith, C.D. (1995) Schizophrenia: Functional imaging and cognitive abnormalities. Lancet, 346, 615-620.

Frith, C.D. (1999) Commentary on Laws: What are we trying to explain? Cognitive Neuropsychology 4, 3132.

Goldman-Rakic, P.S. (1994). Working memory dysfunction in schizophrenia. Journal of Neuropsychiatry and Clinical Neurosciences, 6, 348-357.

Goldstein, G., Beers, S.R., \& Shemansky, W.J. (1996). Neuropsychological differences between schizophrenic patients with heterogeneous Wisconsin Card Sorting Test performance. Schizophrenia Research, 21, 13-18.

Green, M.F. (1996). What are the functional consequences of neurocognitive deficits in schizophrenia? American Journal of Psychiatry, 153, 321-330.

Green, M.F., Kern, R.S., Braff, D.L., \& Mintz, J. (2000). Neurocognitive deficits and functional outcome in schizophrenia: Are we measuring the "right stuff”? Schizophrenia Bulletin, 26, 119-136.

Hegarty, J.D., Baldessarini, R.J., Tohen, M., Waternaux, C., \& Oepen, G. (1994). One hundred years of schizophrenia: A meta-analysis of the outcome literature. American Journal of Psychiatry, 151, 14091416.

Hulstijn, W. (1996) Writing and drawing in neuropsychology: Studies on psychomotor slowing. In: M.L. Simner, C.G. Leedham \& A.J.W.M. Thomassen (Eds.), Handwriting and drawing research: Basic and applied issues (pp. 203-214). Amsterdam: IOS Press.

Hulstijn, W., Jogems-Kosterman, B.J.M., Wezenberg, E., Sabbe, B.G.C. (2001). An evaluation of the use of figure-copying tasks in studies of planning deficits in schizophrenia. In: R.G.J. Meulenbroek \& B. Steenbergen (Eds.), Proceedings of the tenth biennial conference of the International Graphonomics Society (pp. 46-51). Nijmegen: IGS.

Huston, P.E., Shakow, D., \& Riggs, L.A. (1936). Studies of motor function in schizophrenia: II. Reaction time. Journal of General Psychology, 16, 39-82.

Jones, P., Rodgers, B., \& Murray, R., \& Marmot, M. (1994). Child development risk factors for adult schizophrenia in the British 1946 birth cohort. Lancet, 344, 1398-1402.

Johnstone, E.C. (1991). The nature and management of schizophrenia. Scottish Medical Journal, 36, 4-5.

King, H.E. (1991). Psychomotor dysfunction in schizophrenia. In: S.R. Steinhauer, J.H. Gruzelier \& J. Zubin (Eds.), Handbook of schizophrenia, Vol. 5: Neuropsychology, psychophysiology and information processing (pp. 273-301). Amsterdam: Elsevier Science Publishers.

Kraepelin, E. (1919). Dementia praecox and paraphrenia (transl. R.M. Barclay, ed. G.M. Robertson, 1971). Huntington, NY: Robert E. Krieger Publishing. 


\section{8 | Chapter 1}

Lezak, M.D. (1995). Neuropsychological assessment. (3rd edition). Oxford: University Press.

Liddle, P.F. (1987). Schizophrenic syndromes, cognitive performance and neurological dysfunction. Psychological Medicine, 17, 49-57.

Liddle, P.F., \& Morris, D.L. (1991). Schizophrenic syndromes and frontal lobe performance. British Journal of Psychiatry, 158, 340-345.

Liddle, P.F. (1993). The psychomotor disorders: Disorders of the supervisory mental processes. Behavioral Neurology, 6, 5-14.

Loonen, A.J.M., Doorschot, C.H., Van Hemert, D.A., Oostelbos, M.C.J.M., Sijben, A.E.S., \& the MASEAS Team (2000). The Schedule for the Assessment of Drug-Induced Movement Disorders (SADIMoD): Test-retest reliability and concurrent validity. International Journal of Neuropsychopharmacology, 3, 285-296.

Loonen, A.J.M., Doorschot, C.H., Van Hemert, D.A., Oostelbos, M.C.J.M., Sijben, A.E.S., \& the MASEAS Team (2001). The Schedule for the Assessment of Drug-Induced Movement Disorders (SADIMoD): Inter-rater reliability and construct validity. International Journal of Neuropsychopharmacology, 4, 347360.

Luria, A.R. (1980). Neuropsychology in the local diagnosis of brain damage. Clinical neuropsychology, 2, 17.

Mahurin, R.K., Velligan, D.I., \& Miller, A.L. (1998). Executive-frontal lobe cognitive dysfunction in schizophrenia: A symptom subtype analysis. Psychiatry Research, 79, 139-149.

Malla, A.K., Norman, R.M.G., Aguilar, O., Carnahan, H., \& Cortese, L. (1995). Relationship between movement planning and psychopathology profiles in schizophrenia. British Journal of Psychiatry, 167, 211-215.

Murray, R.M., \& Lewis, S.W. (1987). Is schizophrenia a neurodevelopmental disorder? British Medical Journal, 295, 681-682.

Nelson, H.E., Pantelis, C., Carruthers, K., Speller, J., Baxendale, S., \& Barnes, T.R.E. (1990). Cognitive functioning and symptomatology in chronic schizophrenia. Psychological Medicine, 20, 357-365.

Owens, D.G., Johnstone, E.C., \& Frith, C.D. (1982). Spontaneous involuntary disorders of movement: Their prevalence, severity, and disturbance in chronic schizophrenics with and without treatment with neuroleptics. Archives of General Psychiatry, 39, 452-461.

Palmer, B.W., \& Heaton, K. (2000). Executive dysfunction in schizophrenia. In: T. Sharma \& Ph. Harvey (Eds.), Cognition in schizophrenia: Impairments, importance and treatment strategies (pp. 51-72). New York: Oxford University Press.

Pantelis, C., Barnes, T.R.E., Nelson, H.E., Tanner, S., Weatherley, L., Owen., A.M., \& Robbins, T.W. (1997). Frontal-striatal cognitive deficits in patients with schizophrenia. Brain, 120, 1823-1843.

Parkin, A.J. (1996). Explorations in cognitive neuropsychology. Oxford: Blackwell Publishers.

Poole, J.H., Ober, B.A., Shenaut, G.K., \& Vinogradov, S. (1999). Independent frontal-system deficits in schizophrenia: Cognitive, clinical, and adaptive implications. Psychiatry Research, 85, 161-176.

Ram, R., Bromet, E.J., Eaton, W.W., Pato, C., \& Schwartz, J.E. (1992). The natural course of schizophrenia: A review of first-admission studies. Schizophrenia Bulletin, 18, 185-207.

Sabbe, B.G.C., Van Hoof, J.J.M., Hulstijn, W., \& Zitman, F.G. (1996a). Changes in fine motor retardation in depressed patients treated with fluoxetine. Journal of Affective Disorders, 40, 149-158. 
Sabbe, B.G.C., Hulstijn, W., Van Hoof, J.J.M., \& Zitman, F.G. (1996b). Fine motor retardation in depression. Journal of Psychiatric Research, 30, 295-306.

Strauss, M.E. (1993). Relations of symptoms to cognitive deficits in schizophrenia. Schizophrenia Bulletin, 19, 215-231.

Thomassen, A.J.W.M., \& Tibosch, H.J.C.M. (1991). A quantitative model of graphic production. In: Stelmach, G.E., Requin, J. (Eds.), Tutorials in Motor Neuroscience (pp. 269-282). Dordrecht: Kluwer.

Thomassen, A.J.W.M., Meulenbroek, R.G.J., \& Tibosch, H.J.C.M. (1991). Latencies and kinematics reflect graphic production rules. Human Movement Science, 10, 271-289.

Van Hoof, J.J.M., Hulstijn, W., Van Mier, J.I.A.J., \& Pagen, M. (1993). Fine drawing and psychomotor retardation: Preliminary results. Journal of Affective Disorder, 29, 263-266.

Van Hoof, J.J.M. (2002). The abnormal development of drive and guidance mechanisms in the brain: The pathogenesis of schizophrenia. Acta Neuropsychiatrica, 14, 134-146.

Van Hoof, J.J.M. (2003). A motor hypothesis of the origin of schizophrenia (Letter to the editors). Schizophrenia Research, 62, 183-185.

Van Mier, J.I.A.J. (1992). Motor planning and movement disorders: Effects of complexity and practice in drawing tasks. PhD. Dissertation. The Netherlands: University of Nijmegen.

Walker, E.F., Savoie, T., \& Davis, D. (1994). Neuromotor precursors of schizophrenia. Schizophrenia Bulletin, 20, 441-451.

Wechsler, D. (1956). Manual for the Wechsler Adult Intelligence Scale. New York: Psychological corporation.

Weinberger, D.R. (1987). Implications of normal brain development for the pathogenesis of schizophrenia. Archives of General Psychiatry, 44, 660-669.

Widlöcher, D., \& Hardy-Bayle, M.C. (1989). Cognition and control of action in psychopathology. European Bulletin of Cognitive Psychology, 9, 583-615.

Willingham, D.B. (1998). A neuropsychological theory of motor skill learning. Psychological Review, 105, 558-584.

Withaar, F., \& Arends, J. (2002). Cognitive rehabilitation in schizophrenia. In: W. Brouwer, E. van Zomeren, J. Berg, A. Bouma \& E. de Haan (Eds.), Cognitive rehabilitation: A clinical neuropsychological approach (pp. 125-142). Amsterdam: Boom Publishers.

Zec, R.F. (1995). Neuropsychology of schizophrenia according to Kraepelin: Disorders of volition and executive functioning. European Archives of Psychiatry and Clinical Neuroscience, 245, 216-223. 
Chapter 2

\title{
Differentiation of cognitive and motor slowing in the Digit Symbol Test (DST): Differences between depression and schizophrenia ${ }^{1}$
}

\begin{abstract}
Schizophrenia and depression have an overlap in symptomatology, namely a slowing in both motor and mental activities, denoted in depression as 'psychomotor retardation' and in schizophrenia as 'psychomotor poverty'. By means of a new technique that allows the measurement of psychomotor speed and the computerized analysis of writing movements recorded during the performance of the Digit Symbol Test, it indeed proved to be possible to observe a slowing in both disorders. In addition, a different structure of slowing in the two patient groups could be identified.
\end{abstract}

\footnotetext{
${ }^{1}$ Based on:
}

Van Hoof, J.J.M., Jogems-Kosterman, B.J.M., Sabbe, B.G.C., Zitman, F.G., \& Hulstijn, W. (1998). Journal of Psychiatric Research, 32, 99-103. 


\section{Introduction}

In several neuropsychiatric disorders such as depression, schizophrenia and Parkinson's disease a slowing in both motor and mental activities can be observed (Benson, 1990), which has been denoted as psychomotor retardation, psychomotor poverty, or bradykinesia. The question whether the slowing in these different patient groups is identical in nature is, as yet, unresolved (see also Hulstijn, 1996; Van Hoof et al., 1994). This question is not only of theoretical interest (Bermanzohn \& Siris, 1992), but also has great clinical relevance. For instance, a patient who has recovered from acute psychosis may still demonstrate a marked slowing. The question then arises whether this slowing is part of the schizophrenic process or, alternatively, a symptom of a concomitant depression, or, thirdly, a side effect caused by antipsychotic medication.

Psychomotor slowing may have quite different causes. Widlöcher and Hardy-Bayle (1989) hypothesized that in depression slowing is primarily the result of a dysfunction in activation, manifesting itself at different levels of functioning, involving both cognitive and motor processes. They further hypothesized that in schizophrenia the slowing is primarily a 'planning' disorder resulting in cognitive slowing only. More careful testing might shed light on the different causes of psychomotor slowing.

One of the frequently used tests to measure psychomotor slowing is the Digit Symbol Test (DST), a very short subtest of the Wechsler Adult Intelligence Scale (Wechsler, 1956), in which subjects have to substitute symbols for digits according to a key given at the top of the form. In the two psychiatric diseases schizophrenia and depression, the performance on the DST has been found to be impaired. In a comparative study of endogenous and neurotic depressed patients, Austin et al. (1992) found that the performance on the DST was most impaired in the endogenous patients. In schizophrenia the poor performance on the DST was found to be related to negative symptoms and hypofrontality (Wolkin et al., 1992).

The Digit Symbol Test owes its clinical sensitivity to the fact that it makes demands on several processes, i.e. perception, working memory, sustained attention and visuomotor coordination. For a successful completion of the task all these processes need to function properly (Lezak, 1983). The DST, however, has the disadvantage that it gives only one score, which makes it impossible to identify the contributions of the separate processes. In order to try and differentiate between the motor component of the task, i.e. visuomotor coordination, and the cognitive components, i.e. perception, working memory and sustained attention, we used a relatively new recording and analyzing technique in the present study. The DST was administered on a digitizing tablet allowing the precise recording of the pen movements made during the test. This technique (Teulings \& Maarse, 1984) has a long history in the study of writing and drawing (Thomassen \& Van Galen, 1992), but has only recently been applied in research in psychiatry (Van Hoof et al., 1993; Hulstijn, 1996; Sabbe et al., 1996a, 1996b, 1997). It allows a differentiation of 'writing time', i.e. the time taken to write a symbol, and what is often called 'matching time', i.e. the intervals between the writing of the successive symbols, which is the time needed to find the right symbol, or the time to 'match' the current digit with the proper symbol.

In this study we first investigated whether, by using this method, a slowing on the DST could be replicated in both depression and schizophrenia. To this end groups of depressed and schizophrenic 
patients were compared with each other and with a group of healthy control subjects. The main objective, however, was to explore the relative contributions of the motor and cognitive components to the slowness in both patient groups. It was hypothesized that in depression both motor and cognitive processes are slowed resulting in a delay in matching as well as writing time, while schizophrenic patients would only display cognitive slowing and no delay in writing time.

\section{Method}

\section{Subjects and design}

Two groups, each of 20 psychiatric inpatients, participated in this study. The first group consisted of depressed patients meeting DSM-III-R criteria for major depression (American Psychiatric Association, 1987). All depressed patients were tested on admission to the clinic. As the symptomatology of the depression is more severe at the beginning of the hospital stay, we preferred to test the depressed patients during the acute phase. Eleven of them were already taking the antidepressant fluoxetine. With the remaining nine patients antidepressant treatment had not yet been started. In addition, some patients took low dosages of benzodiazepines $(n=8)$, neuroleptics $(n=2)$ or a combination of the two $(n=2)$.

The second group consisted of 20 schizophrenic patients, according to DSM-III-R criteria (American Psychiatric Association, 1987). All schizophrenic patients were in a stable phase of their disease and were treated with neuroleptic medication. Fifteen of these patients were taking typical neuroleptics, two patients received atypical neuroleptics and one patient was treated with both types of medication. From the remaining two patients medication was not registrated. For 12 patients chlorpromazine equivalents could be calculated. These ranged from 100-2250, with a mean of 710 . Some patients were also taking low dosages of anticholinergics $(n=4)$, benzodiazepines $(n=6)$ or both $(n=7)$. However, because of the likelihood that psychotic symptoms would interfere with task performance, we decided to test the schizophrenic patients in a more stable phase of their disorder following the acute phase.

Twenty healthy subjects, matched for sex and age were used as controls. The controls mainly originated from the general public. A number of some control subjects were locally recruited among the (lower) hospital staff (see table 1). Level of education was not found to have any effect on the performance of the three groups.

All patients were scored on the Hamilton Depression Rating Scale (Hamilton, 1960). The severity of the psychomotor retardation in the patients was assessed by means of the Salpetrière Retardation Rating Scale (Widlöcher, 1983). The cut-off score for the Salpetrière Retardation Scale is 20. For the mean scores on these scales, see Table 1. The Hamilton depression scores were significantly higher in the depression group than in the schizophrenia group $(p<0.001)$. No significant difference between the groups was found on the Salpetrière Retardation Rating Scale. 


\section{4 | Chapter 2}

Table 1: Number of subjects, distribution of sex, mean age and mean scores on the Hamilton Depression Rating Scale (HDRS) and the Salpetrière Retardation Rating Scale (SRRS).

\begin{tabular}{llll}
\hline & Depression & Schizophrenia & Control \\
\hline Number & 20 & 20 & 20 \\
Sex (male; female) & $10 ; 10$ & $10 ; 10$ & $10 ; 10$ \\
Age in years (SD) & $47.0(12.1)$ & $39.9(12.2)$ & $43.1(13.5)$ \\
HDRS 17 items (SD) & $24.8(6.2)$ & $12.8(5.0)$ & - \\
SRRS (SD) & $29.9(10.1)$ & $22.7(10.7)$ & - \\
\hline
\end{tabular}

\section{Task and apparatus}

All subjects performed the Dutch version of the Digit Symbol Task (DST), which is a subtest of the Wechsler Adult Intelligence Scale (WAIS) (Wechsler, 1956). In this task a series of symbols has to be decoded as fast as possible during 90 seconds by using a key consisting of nine symbols and the numbers 1 to 9 . In contrast to the English version of the DST, in the Dutch version numbers have to be filled in instead of symbols.

In this study, the DST form was placed on a Calcomp 2500 digitizing tablet. This apparatus, in combination with a special pressure-sensitive ball-point pen, allows the registration of the $\mathrm{x}$ and $\mathrm{y}$ coordinates of the pen tip when in contact with the digitizer as well as up to approximately $5 \mathrm{~mm}$ above the writing surface. The position of the pen and the axial force exerted on the tip of the pen were sampled with a frequency of $100 \mathrm{~Hz}$ and a spatial accuracy of $0.2 \mathrm{~mm}$. After completion of the task, the recorded signals were filtered by means of a Fast-Fourier analysis. See Teulings and Maarse (1984) for more details about this method.

\section{Analysis}

The total registration period of 90 seconds was automatically segmented on velocity minima. By means of an interactive computer program and based on pen pressure, these segments were defined as either pen-up periods or pen-down periods (pressure threshold: 24 gr). Furthermore, pen-down periods during which the movement velocity remained below $1 / 5$ of the mean velocity were defined as pause intervals, whereas the remaining pen-down periods were defined as movement time.

Dependent variables were (1) raw score, i.e. the number of correct digits produced within 90 seconds, (2) matching time, operationalized as the accumulated pen-up periods between two successive digits and the pause intervals at the end and at the beginning of these digits, (3) writing time, operationalized as the time between the first and final movement within a digit, and (4) distance, operationalized as the distance covered by the pen during the pen-down periods. Matching time, writing time, as well as distance were calculated as a mean value per digit. ${ }^{2}$

Comparisons were made by means of analyses of variance (ANOVA) with one betweensubject factor, viz. group (depression, schizophrenia, control).

\footnotetext{
${ }^{2}$ In the calculation of the kinematic variables the first digit on each new line was not taken into account, because of the large distance that had to be covered by the pen.
} 


\section{Results}

The results of the ANOVAs obtained for the different variables are displayed in Table 2.

Table 2: Mean values, standard deviations and results of analyses of variance for raw score, mean matching time per digit, mean writing time per digit and mean distance per digit for the depressed patients, the schizophrenic patients and controls.

\begin{tabular}{|c|c|c|c|c|c|}
\hline Variable & Group & & & Statistic & P-level \\
\hline & Depression & Schizophrenia & Control & & \\
\hline $\begin{array}{l}\text { Raw Score (number of digits } \\
\text { during } 90 \mathrm{~s} \text { ) }\end{array}$ & $39.95(16.21)$ & $32.60(13.89)$ & $53.55(14.93)$ & $F(2,59)=10.20$ & $\mathrm{p}<0.001$ \\
\hline Mean matching time per digit (s) & $1.77(0.78)$ & $2.25(0.80)$ & $1.29(0.61)$ & $F(2,59)=8.57$ & $\mathrm{p}<0.002$ \\
\hline Mean writing time per digit (s) & $0.68(0.31)$ & $0.57(0.15)$ & $0.50(0.11)$ & $F(2,59)=4.04$ & $\mathrm{p}<0.03$ \\
\hline Mean distance per digit $(\mathrm{cm})$ & $1.26(0.23)$ & $1.24(0.34)$ & $1.33(0.59)$ & $F(2,59)=0.23$ & $\mathrm{p}=0.797$ \\
\hline
\end{tabular}

Duncan tests (with $\alpha$ set at 0.05 ) were applied to investigate the differences between the three groups more closely. Compared to the controls both the depressed and the schizophrenic patient groups performed worse, i.e. they had a lower raw score on the DST. All groups differed significantly in matching time. Schizophrenic patients displayed a significantly prolonged matching time per digit as compared to the two other groups. In matching, the depressed patients were faster than the schizophrenic patients, but slower than the controls. In writing time per digit, only the depressed patients displayed a significant slowing as compared to the controls.

Furthermore, we found that the relative contribution of the mean matching and writing time to the mean total time per digit was significantly different (Duncan test, $\alpha=0.05$ ) in the depressed patients $(71.3 \%$ and $28.7 \%$, respectively) and the controls $(70.2 \%$ and $29.8 \%)$ as compared to the schizophrenic patients $(78.9 \%$ and $21.1 \%)$. This indicates that a general slowing, i.e. in both matching and writing, occurred in the depressed patients when compared to the controls, whereas it is remarkable that the slowing found in the schizophrenia group was more restricted to matching than writing when compared to both the depressed patients and the controls.

\section{Discussion}

By means of a new technique, namely the computerized analysis of writing movements, this study shows that both depressed and schizophrenic patients are slower on the DST than the controls. Our study replicates results from earlier studies using DST. The data of the depressed patient group reconfirm our earlier results of a manifest psychomotor slowness in depressed patients (Van Hoof et al., 1993; Sabbe et al., 1996a, 1996b, 1997). 
Our second objective was to investigate whether this technique could identify a difference in the relative contributions of the motor and cognitive components of the slowness in both patient groups. This was indeed the case. The depressed patients demonstrated an overall slowing manifesting itself in significantly prolonged matching and writing times as compared to the controls. In contrast, the schizophrenic patients only showed a significantly prolonged matching time in comparison with both the depressed patients and the controls.

Since the writing of a digit is a more or less automatic process, the time the pen is moving on the tablet (writing time) is assumed to represent motor activities such as visuomotor coordination. On the other hand, the intervals during which the subject pauses above and on the writing tablet between two successive digits (matching time) are assumed to incorporate higher processes such as attention, perception, memory and programming. These assumptions support our interpretation that, compared to the controls, the depressed patients showed a slowness in both motor and cognitive components whereas the schizophrenic patients only exhibited a slowness in the cognitive component. Consequently, our analysis shows that the slowing found in depressed patients cannot simply be put on the same level as the slowing observed in schizophrenic patients. It is probable that in schizophrenia primarily the higher processes such as retrieval of motor programs, parameterization and initiation are affected. This can be related to the hypofrontality mentioned above. In depression, however, not only higher (cognitive) processes are affected, but also motor processes. Our interpretation is in line with the theory of Widlöcher and Hardy-Bayle (1989) who hypothesized that depression is primarily a dysfunction in activation, manifesting itself at different levels of functioning involving both cognitive and motor processes. They also proposed that in schizophrenia processes such as planning and programming seem to be affected.

It is unlikely that the slowing we observed in the present study can be attributed to the use of fluoxetine, the antidepressive drug the depressed patient group was treated with. This drug is not associated with persistent psychomotor dysfunctions. Furthermore, no adverse effects of the comparable drug sertraline were found on psychomotor performance as assessed by means of the DST (Ravindram et al, 1995). However, generally the psychomotor effects of the use of neuroleptics cannot be neglected. Clinical practice suggests that motor slowing is quite frequently found in patients treated with classical neuroleptics, although no effects or even improvements have also been found (King, 1990). In our study the time to write the digits in the DST was not significantly longer than that of control patients for the total group of schizophrenic patients. In a further analysis, in which the chlorpromazine equivalents of neuroleptic medication was correlated with writing time, writing distance and writing velocity, it was found that a higher dose reduced the writing velocity (Kendall's $\tau$ $=0.49, \mathrm{p}=0.027 ; \mathrm{n}=12$ ) which resulted in a few subjects in a longer writing time and a tendency for micrographia in a few other patients. The two patients treated with atypical neuroleptics had writing speed and duration close to the mean of the other patients. The small numbers of patients in these subgroups precludes firm conclusions, however. As in both patient groups comparable numbers of subjects were taking benzodiazepines, it is unlikely that the differences in performance can be attributed to the use of this medication. In addition, no differences in performance were found between the schizophrenic patients treated with anticholinergics and the patients that did not take this type of medication. It is therefore also improbable that the effect of anticholinergics is relevant for the outcome 
of this study.

Differences between depressed and schizophrenic patients were found. However, the patients were tested at different moments of their stay in the hospital, the depressed patients shortly following their admission during the acute phase of their disease and the schizophrenic patients at a later stage after the acute phase of their disorder. It seems unlikely though, that this could fully explain the differences found in test performance. Follow-up studies that are currently being conducted (JogemsKosterman et al., in preparation) will demonstrate the relevance of the time of testing.

As this study shows once again, the employment of writing tasks, together with the computerized registration of the writing movements in psychiatric research opens up new perspectives. With this method it is possible to differentiate the psychomotor symptoms of different patient groups objectively. It may also facilitate the distinction between subgroups of patients with or without motor slowing. However, in future studies effects of medication should be separated from 'disease symptomatology'. Moreover, we advocate further studies into the possible predictive value of the findings that are obtained with this technique, for instance with respect to effects of treatment.

The unraveling of the different processes involved in the performance of writing and drawing tasks may provide a deeper insight into the impaired information processing in several neuropsychiatric disorders such as depression, schizophrenia, closed head injury, Parkinson's disease and dementia. In addition, the examination of the correlations of functional and (neuro)anatomical abnormalities by means of simultaneous neurophysiological and/or neuroimaging research during writing and drawing tasks may lead to a better understanding of the underlying pathophysiological mechanisms of these intriguing disorders.

\section{Acknowledgements}

Thanks are due to Hans Knoop and Hanno van der Heyden for helping to set up the experiment and for testing a proportion of the subjects. 


\section{References}

American Psychiatric Association (1987). Diagnostic and statistical manual of mental disorders (3rd edition, revised). Washington, D.C.: APA.

Austin, M.P., Ross, M., Murray, C., O’Carroll, R.E., Ebmeier, K.B., \& Goodwin, G.M. (1992). Cognitive function in major depression. Journal of Affective Disorders, 25, 21-29.

Benson, F. (1990). Psychomotor retardation. Neuropsychiatry, Neuropsychology, and Behavioral Neurology, 3, $36-47$.

Bermanzohn, P.C. \& Siris, S.G. (1992). Akinesia: A syndrome common to parkinsonism, retarded depression, and negative symptoms of schizophrenia. Comprehensive Psychiatry, 33, 221-232.

Hamilton, M. (1960). A rating scale for depression. Journal of Neurology, Neurosurgery and Psychiatry, 23, $56-62$.

Hulstijn, W. (1996). Writing and drawing in neuropsychology: Studies on psychomotor slowness. In M.L. Simner, C.G. Leedham, \& A.J.W.M. Thomassen (Eds.), Handwriting and Drawing Research: Basic and Applied Issues (pp. 203-214). Amsterdam: IOS Press.

Jogems-Kosterman, B.J.M., Van Hoof, J.J.M. \& Hulstijn, W. (in preparation). Psychomotor slowness in depression: A follow-up study.

King, D.J. (1990). The effects of neuroleptics on cognitive and psychomotor function. British Journal of Psychiatry, 157, 799-811.

Lezak, M.D. (1983). Neuropsychological Assessment (2nd edition). New York: Oxford University Press.

Ravindram, A.V., Teeham, M.D., Bakish, D., Yatham, L., O'Reilly, R., Fernando, M.L., Manchanda, R., Charonneau, Y., \& Buttars, J. (1995). The impact of sertraline, desipramine and placebo on psychomotor functioning in depression. Human Psychopharmacology, 10, 273-281.

Sabbe, B.G.C., Hulstijn, W., Van Hoof, J.J.M., \& Zitman, F.G. (1996a). Fine motor retardation and depression. Journal of Psychiatric Research, 30, 295-306.

Sabbe, B.G.C., Van Hoof, J.J.M., Hulstijn, W., \& Zitman, F.G. (1996b). Changes in fine motor retardation in depressed patients treated with fluoxetine. Journal of Affective Disorders, 40, 149-157.

Sabbe, B.G.C., Hulstijn, W., Van Hoof, J.J.M., \& Zitman, F.G. (1997). Depressive retardation and treatment with fluoxetine: Assessment of the motor component. Journal of Affective Disorders, 43, 53-62.

Teulings, H., \& Maarse, F.J. (1984). Digital recording and processing of handwriting movements. Human Movement Science, 3, 193-217.

Thomassen, A.J.W.M., \& Van Galen, G.P. (1992). Handwriting as a motor task: Experimentation, modeling, and simulation. In: J.J. Summers (Ed.), Approaches to the study of motor control and learning (pp. 113-144). Amsterdam: North Holland.

Van Hoof, J.J.M., Hulstijn, W., Van Mier, H., \& Pagen, M. (1993). Figure drawing and psychomotor retardation: Preliminary report. Journal of Affective Disorders, 29, 263-266.

Van Hoof, J.J.M., Sabbe, B.G.C., Hulstijn, W., Kosterman, B.J.M., Loonen, A.J.M., \& Zitman, F.G. (1994). The specificity question of retardation and depression. In: R.D. Ciaranello \& H.Y. Meltzer (eds.), Neuropharmacology, 10, 745S. Washington DC: Elsevier.

Wechsler, D. (1956). Manual for the Wechsler Adult Intelligence Scale. New York: Psychological corporation. Widlöcher D.J. (1983). Retardation: A basic emotional response? In: J.M. Davis \& J.W. Maas (eds.), The affective disorders (pp. 165-181). Washington, D.C.: American Psychiatric Press. 
Differentiation of cognitive and motor slowing in the Digit Symbol Test (DST) | 39

Widlöcher, D.J., \& Hardy-Bayle, M.C. (1989). Cognition and control of action in psychopathology. European Bulletin of Cognitive Psychology, 9, 583-615.

Wolkin, A., Sanfilipo, M., Wolf, A.P., Angrist, B., Brodie, J.D., \& Rotrosen, J. (1992). Negative symptoms and hypofrontality in chronic schizophrenia. Archives of General Psychiatry, 49, 959-965. 


\title{
Chapter 3
}

\section{Antipsychotics, movement disorders and fine motor performance: New device for the measurement of bradykinesia and tremor ${ }^{1}$}

\begin{abstract}
This paper reviews the results of an exploratory study on the relationship between the ratings of bradykinesia, obtained by the Schedule for the Assessment of Drug-Induced Movement Disorders (SADIMoD), and the performances on writing and drawing tasks. The pen movements made during these tasks were recorded by means of an electronic digitizing tablet, a special electronic pen and a personal computer. This study was aimed at finding objective parameters for the severity of (antipsychotic-induced) bradykinesia. The results show that the writing tablet device is appropriate for finding movement variables that are related to the clinical ratings for bradykinesia: high rating scores for bradykinesia (SADIMoD) were associated with slowing on a number of duration measures during the execution of the writing and drawing tasks. We were also able to measure (postural) tremor by means of the writing tablet. However, an accelerometer seems to be more appropriate for this purpose.
\end{abstract}

\footnotetext{
${ }^{1}$ Based on:
}

Jogems-Kosterman, B.J.M., Loonen A.J.M., Van Hoof, J.J.M., Zitman, F.G., \& Hulstijn, W. (1998). Acta Neuropsychiatrica, 10, 93-99. 


\section{Introduction}

The use of antipsychotic drugs may be considered as one of the essential requirements for patients with schizophrenia to maintain an adequate level of functioning. The introduction of antipsychotic drugs in the period between 1950 and 1960 has contributed greatly to the dehospitalization of patients with schizophrenia (Davis, 1980). Nevertheless, most doctors are aware of the disadvantages of prescribing antipsychotic drugs. This holds particularly true for the classical neuroleptics that may cause many unpleasant side effects in patients. One of the most compelling disadvantages is the occurrence of motor side effects. For several reasons, i.e. for scientific research aimed at finding the most adequate treatments for patients, for investigating the applicability of new compounds and, besides, for being able to follow individual patients during their treatment, reliable quantitative methods are needed for assessing these motor side effects. Several methods for the assessment of motor side effects are available.

Instruments that already have a long tradition in the scientific field of movement disorders are clinical rating scales, such as the AIMS (Abnormal Involuntary Movement Scale; Guy, 1976) and the EPS (Extrapyramidal Symptoms scale; Simpson \& Angus, 1970). The disadvantage of most of these scales, however, is that they measure distinct movement disorders (Loonen \& Zwanikken, 1987). The recently developed Schedule for the Assessment of Drug-Induced Movement Disorders (SADIMoD) is constructed out of a number of existing rating scales, that enable the rating of movement disorders simultaneously and independently from each other (Loonen \& Doorschot, 1994, 1996). Moreover, the SADIMoD enables a more differentiated judgement, and the different movement disorders are more sharply defined. A disadvantage of the SADIMoD, when compared to other instruments, is that it is quite time-consuming to administer the scale (30-45 min) and that it requires well-defined conditions, such as the size of the examination room and the need of video recording.

Besides the use of clinical rating scales, there is a growing interest in the use of instrumental or electronic instruments. Most of these studies have provided objective parameters for rigidity and/or tremor (Webster, 1966; Martinez, 1966; Boshes, 1966; Nashold, 1966; Caliguiry et al., 1989; Teräväinen et al., 1989; Caliguiry et al., 1991; Caliguiry, Lohr \& Jeste, 1993).

Another method is the measurement of the speed and precision of particular well-defined movements. Under experimental conditions, movements produced during a number of writing or drawing tasks are recorded with the aid of an electronic pen, a digitizing tablet and a personal computer, and are analyzed by means of a special software package, called OASIS. This method has been developed within our institutes for the measurement of psychomotor slowing in depression and schizophrenia (Van Hoof et al., 1996; Sabbe et al., 1996; Van Hoof et al., 1998 and the foregoing Chapter of the present thesis).

It has been already known for many years that the assessment of patients' handwriting can be helpful in the observation of the severity of motor side effects of antipsychotic drugs (Haase, 1977). For this reason, a writing test has been included in the previously mentioned SADIMoD. However, it is not only important to judge the product as the end result of writing, but it can also be very informative to assess the process of writing. These analyses will probably provide useful 
parameters for the intensity of a number of movement disorders that are frequently seen in patients treated with antipsychotic drugs or in chronic psychiatric patients.

For its application in a clinical setting, an instrument has to be little stressful for the patient and very user-friendly for the investigator, so that an examination can be performed by an investigator with little training during a regular visit of the patient to the outpatient clinic. With this in mind, we are engaged in an attempt to develop an experimental setting for the quantification of particular movement disorders.

In this paper we describe the results of our study on the relationship between the severity ratings of movement disorders (mostly due to the use of antipsychotic drugs) and the performances on fine motor tasks. The study was in particular aimed at quantifying bradykinesia as part of parkinsonism, as the method we use is particularly appropriate for the measurement of time durations (including movement velocity, movement distance covered, and duration of pauses) during task performance.

The present study addresses the following two questions:

1) Is there a relationship between the performance on fine motor tasks and ratings of antipsychoticinduced bradykinesia?

2) Which movement components (initiation time, movement time, duration of pauses, movement distance etc.) are most closely related to this bradykinesia?

We have selected a number of simple and patient-friendly writing and drawing tasks. During the performance of these tasks, pen movements made on and above the digitizing tablet were recorded. In addition, an accelerometer was applied in order to measure tremors. We also tried to quantify tremors, rigidity and ataxia in an exploratory way with the aid of the digitizer.

\section{Method}

\section{Subjects}

Potential subjects were male and female patients, aged between 17 and 65 years, who were admitted to one of the wards of GGZ Oost Brabant (sites Coudewater and Huize Padua) on a voluntary basis. The patients were diagnosed as having a psychotic disorder, primarily schizophrenia according to DSM-III-R criteria. They did not suffer from an organic or affective psychosis, and they did not have a physical or mental disability that could interfere with task performance. They were neither seriously agitated nor suspicious. Initially, certain patients were asked by their treating physician whether they would participate in the study. In these patients, the physician had observed one or more of the following movement disorders: bradykinesia, tremor(s), rigidity or ataxia. Subsequently, patients received verbal and written information from the investigator.

Sixteen patients (seven men and nine women) participated in the study. All these patients gave their informed consent. Prior to the start of the study, the medical-ethical committee of the South part of the Netherlands (Toetsingscommissie Zuid-Nederland; TCZ) gave her approval for the carrying out of this study. 


\section{Design}

The physician introduced those patients into the study who suffered from at least one of the mentioned movement disorders with moderate severity. After judgement of the investigator, patients were examined once by means of the clinical rating scale and the fine motor tasks. If possible, all parts of the examination were conducted on the same day. When necessary, for example to prevent the patient from being overloaded, the examination was spread out over separate days. In this case, the inter-day variability and the severity of the movement disorders were clinically scored and checked.

\section{Clinical rating scale}

Movement disorders were rated by means of the Schedule for the Assessment of Drug Induced Movement disorders (SADIMoD; Loonen \& Doorschot, 1994). The SADIMoD consists of a compilation of several rating scales, assessing the following movement disorders: dyskinesia, dystonia, akathisia, parkinsonism, tremor and ataxia. The patient was submitted is to a strictly standardized examination, which was videotaped according to prescribed instructions. The SADIMoD contains a glossary in which the different movement disorders are described, classified and defined and in which criteria are given for the severity scores.

\section{Apparatus, experimental tasks and procedure}

Digitizing tablet: Patients performed a number of writing and drawing tasks while pen movements were recorded by means of an electronic digitizing tablet (WACOM 1218RE), a normal looking electronic pen and a personal computer. See Figure 1 for the test situation. The position of the pen on (and up to $5 \mathrm{~mm}$ above) the digitizing tablet was sampled with a frequency of $200 \mathrm{~Hz}$ and a spatial accuracy of $0.2 \mathrm{~mm}$. Also the axial pressure exerted on the pen tip was recorded. The writing and drawing tasks were performed on normal sheets of paper (A4-format) that were put on the tablet.

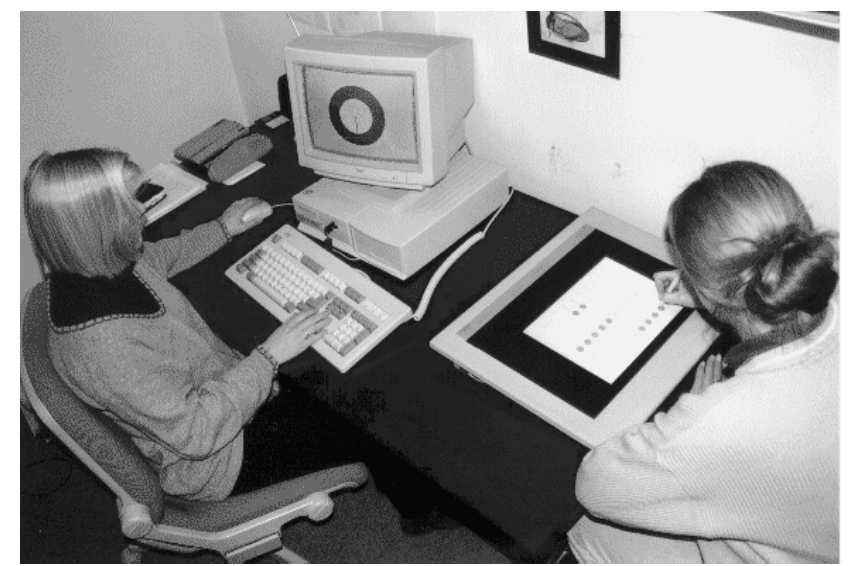

Figure 1: Test situation. 
The following tasks were used in the present study.

Writing task: The instruction was to copy a Dutch sentence ('Ik schrijf deze zin keer op keer'/'I write this sentence over and over') five times at a fast speed and in one's own handwriting style. In accordance with previous research, we chose this task because bradykinesia may cause slowness of writing and micrographia.

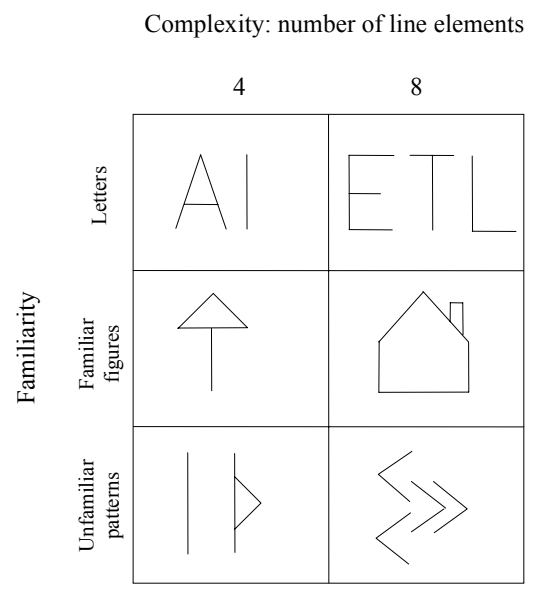

Figure 2: Examples of stimuli used in the figure-copying task.

Aiming task: Subjects were instructed to trace as fast as possible a downward line from a black spot (Ø $0.2 \mathrm{~cm}$ ) towards a grey target (two target widths: $\varnothing 0.375 \mathrm{~cm}$ and $\varnothing 1.5 \mathrm{~cm}$ ). The distance between the middle of the black spot and the middle of the grey target was $3 \mathrm{~cm}$. The task consisted of 24 trials, with the first four trials serving as practice. In this task, a distinction could be made between the starting period (initiation time) and the execution period of the movement (movement time, movement velocity). It had appeared earlier that patients with Parkinson's disease are slowed down in both the starting (from a standstill) and the execution of a movement. With respect to the latter, patients with Parkinson's disease appear to execute goal-directed movements in several smaller steps (Marsden, 1989).

Figure-copying task: The instruction was to copy graphic stimuli that were presented on the computer screen as fast and as accurately as possible. In this task the complexity of the stimuli was varied systematically. The stimuli were either letters, (familiar) figures or (unfamiliar) patterns. These three categories were further divided into simple (four-line elements) and complex (eight-line elements) stimuli (Van Hoof et al., 1996). This task was aimed at investigating the relationship between the complexity of stimuli - and the involved task performance - and the severity ratings of the bradykinesia. The task contains 12 trials in which stimuli are presented. See Figure 2 for some of examples of the stimuli. The stimuli had to be copied in rectangles $(3 \mathrm{x} 4 \mathrm{~cm})$ that were provided on the paper sheets. To start the trial, the subject was instructed to put the pen tip in a circle at the 
lower left corner of the rectangle. After approximately one second, the stimulus was presented on the screen, which was accompanied by a 'go' signal. As soon as the subject put the pen on the paper and started drawing, the stimulus disappeared from the screen. During drawing, but only if necessary, a subject could reinspect the stimulus on the screen. To achieve this, the pen had to be repositioned in the starting circle. As soon as the subject restarted drawing, the stimulus disappeared again.

Circle task: This task consisted of producing circular or eight-shaped movements during 15 seconds. The movements have to be drawn within the boundaries of circular or eight-shaped grey tracks presented on the paper. In the case of a small circle or eight-shape, the radius was $3.5 \mathrm{~cm}$ and the width of the grey track was $0.5 \mathrm{~cm}$. In the case of the bigger circle and eight-shape, these numbers were 7 and $1 \mathrm{~cm}$, respectively. The instruction was to produce movements within the grey track as fast as possible. As this task consists of the production of continuous circular movements, it is very appropriate to measure tremors. In other graphic tasks, such as writing or shading, the changes in movement velocity imposed by the task would interfere too much with the tremor frequency.

Accelerometer: Patients performed three tasks with the preferred hand, while an accelerometer was attached to the middle finger. We used a small accelerometer of ICSensor, type 3021-005, with a sensitivity range of 5G. First, the patient was asked to sit with the hands resting loosely on their lap. Then arms, hands and fingers had to be stretched forward. Finally, the patient was asked to point at several targets (circles) successively over a distance of $30 \mathrm{~cm}$. These targets were indicated on an A3-format sheet. These tasks were performed while sitting on a normal chair. Each task consisted of three registration periods of 15 seconds.

\section{Data recording and analysis}

The data from the digitizer were recorded and analyzed by means of the software package OASIS (Optimized Action Sequence Interpreter System) which was developed for research on handwriting and fine motor performance (De Jong et al., 1996).

In the present study, averaged over all trials, a number of movement variables were determined. For each task was calculated: movement time (MT), the time (sec) the pen was moving on the paper (velocity $>0.57 \mathrm{~cm} / \mathrm{sec})$; mean velocity $(\mathrm{MV})$ : mean velocity $(\mathrm{cm} / \mathrm{sec})$ of the pen moving on the paper; distance $(\mathrm{cm})$ covered on the paper (Dis): pausing time (PT), the time (sec) the pen was standing still on the paper (velocity $<0.57 \mathrm{~cm} / \mathrm{sec}$ ). For the writing task and the figurecopying task also pen-up time (UpT) was determined: i.e. the time (sec) the pen was above the paper during the execution of movements. Initiation time (IT), the time (sec) between the warning signal and the first pen movement on the paper, was determined for the aiming task and the figurecopying task.

For the registration and analysis of the accelerometer data we used software program Testpoint 1.1 (for Windows). The amplified signal was digitized, on $200 \mathrm{~Hz}$, by means of a DAS 1602 data acquisition card. Signals were filtered by means of a special adaptive filter in order to 
eliminate $50 \mathrm{~Hz}$ buzz. Offset was put at zero. Finally the acceleration signal was transformed to the frequency domain by means of 'Power Spectrum Density Analysis' (PSDA). The term 'power' denotes the amount of energy present in each of a large number of frequency bands.

Also the data from the digitizing tablet could be analyzed by means of the PSDA method. However, this was done for the circle task only, as in this task a continuous movement was made during a sufficient period of time. In order to apply the PSDA method, the (position) signal had to be converted to an acceleration signal. This implies a certain loss of accuracy when compared to the accelerometer that directly measures acceleration.

Correlations (Kendall's $\tau$ non-parametric) were calculated between the different movement variables and the bradykinesia rating scores on the SADIMoD. Given the exploratory character of the measurements of tremor, rigidity and ataxia and our expectation that only few patients would show these disorders, we chose for this second part descriptive statistics.

\section{Results}

\section{Bradykinesia}

The correlations between the rating scores on the item 'bradykinesia' (range 0-4) of the SADIMoD, which is defined as 'slowing and reduction of amplitude of spontaneous, associated and intended movements' and the various movement variables measured during the task performances are shown in table 1. Due to technical problems with the apparatus, some measurements are missing in four patients: those of the figure-copying task in two patients; of the aiming task in another patient; and finally of the writing task in a fourth patient.

Table 1: Correlation coefficients (Kendall's $\tau$ ) between the clinical rating of bradykinesia (assessed by means of the SADIMoD) and the various movement variables.

\begin{tabular}{|c|c|c|c|c|c|c|}
\hline & IT & MT & $\underline{\mathrm{PT}}$ & UpT & MV & Dis \\
\hline Writing task $(\mathrm{n}=15)$ & - & $0.54 *$ & $0.56^{* *}$ & 0.36 & -0.29 & 0.06 \\
\hline Aiming task $(\mathrm{n}=15)$ & $0.52 *$ & 0.39 & $0.44 *$ & - & -0.37 & -0.29 \\
\hline Figure-copying task $(\mathrm{n}=14)$ & 0.19 & 0.39 & 0.34 & 0.24 & $-0.47 *$ & -0.19 \\
\hline
\end{tabular}

$* * \mathrm{p}<0.01, * \mathrm{p}<0.05$

Writing task: bradykinesia was only significantly associated with MT and PT. This means that bradykinesia during writing is particularly linked with the duration of the movements and pausing on the paper.

Aiming task: bradykinesia appeared to correlate significantly with MT and PT. Figure 3 shows the mean values of IT, MT and PT for the different bradykinesia rating scores. Although a clear increase of durations could be found with increasing bradykinesia ratings (score 4 was never 
assigned), the difference between scores 1 and 2 was small and even opposite to what could be expected.

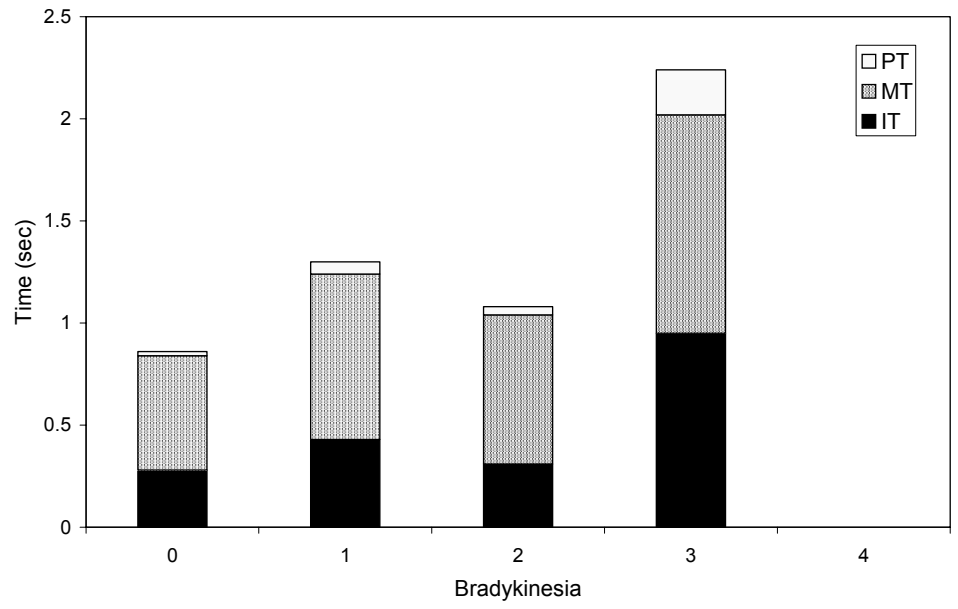

Figure 3: Mean measures of the movement variables in the aiming task for each rating of bradykinesia.

Figure-copying task: bradykinesia correlated significantly only with MV. In this task, in contrast to the aiming task, bradykinesia did not correlate with IT. When in the figure-copying task a differentiation was made between the different levels of cognitive complexity (see Table 2), it appeared that bradykinesia correlated with IT in copying the simple letters. This means that bradykinesia seems to be expressed most clearly in initiating simple tasks (just like in the aiming task). In the copying of more complex figures, cognitive processes, that are necessarily involved, will play a more crucial role in the slower execution of the task.

Table 2: Correlation coefficients (Kendall's $\tau$ ) between the clinical ratings of bradykinesia (assessed by means of the SADIMoD) and the movement variables in the figure-copying task divided over levels of familiarity (letters, figures, patterns) and cognitive complexity ( 4 or 8 strokes).

\begin{tabular}{lrrrrrrr}
\hline & \multicolumn{2}{c}{ Letters } & \multicolumn{2}{c}{ Figures } & \multicolumn{2}{c}{ Patterns } \\
\hline Number of strokes & 4 & 8 & 4 & 8 & 4 & 8 \\
\hline IT & $0.57 * *$ & 0.39 & 0.06 & 0.16 & 0.16 & 0.14 \\
MT & 0.36 & 0.29 & 0.16 & $0.51 *$ & $0.49 *$ & 0.21 \\
PT & 0.41 & $0.49 *$ & 0.19 & 0.32 & 0.34 & 0.19 \\
UpT & 0.11 & 0.39 & -0.01 & 0.27 & 0.09 & 0.14 \\
MV & $-0.44 *$ & -0.42 & -0.42 & $-0.49 *$ & -0.47 & -0.39 \\
Dis & -0.16 & -0.14 & -0.29 & -0.04 & -0.14 & -0.24 \\
\hline
\end{tabular}

$* * \mathrm{p}<0.01, * \mathrm{p}<0.05$ 


\section{Circle task and tremors}

Within the total group of patients only one patient (FK) appeared to show a manifest tremor. This corresponds with the general finding that a tremor, as a side effect of antipsychotic medication, occurs less often than symptoms like bradykinesia and akathisia. This patient obtained a score of 3 on the tremor scale (0-4) of the SADIMoD. This tremor was also clearly seen with the accelerometer while stretching the arms forward. The peak frequency in the tremor of patient FK was clearly higher when compared to the other patients (see Figure 4). Patient FK showed a comparable tremor frequency in the execution of the circle task (see Figure 5).

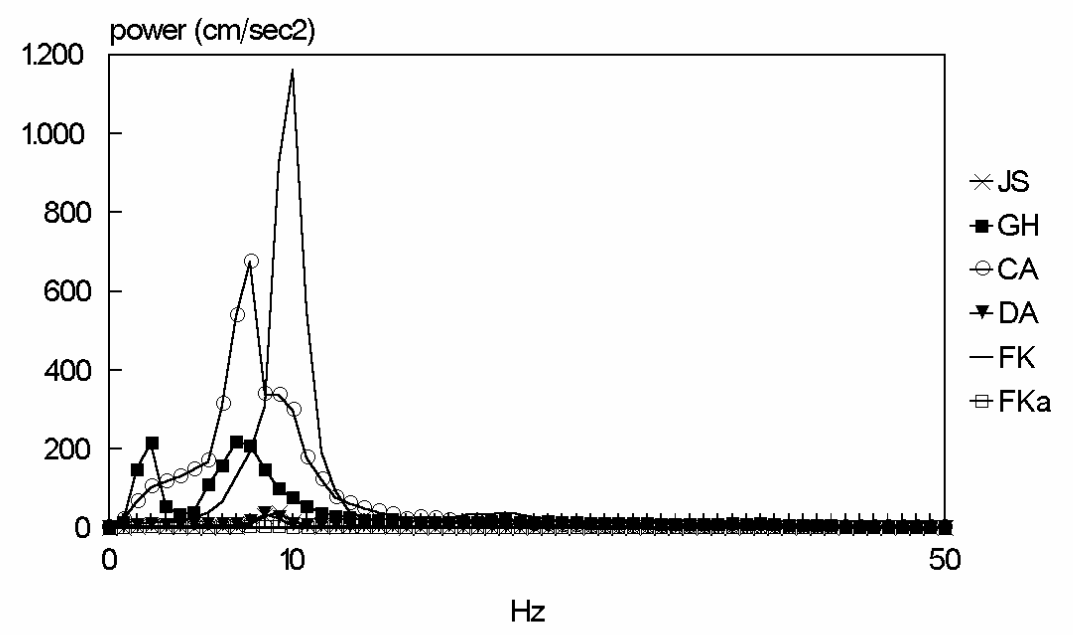

Figure 4: A Power Spectral Density Analysis (PSDA) of the acceleration signal obtained with the accelerometer during $15 \mathrm{sec}$. while stretching the arms horizontally.

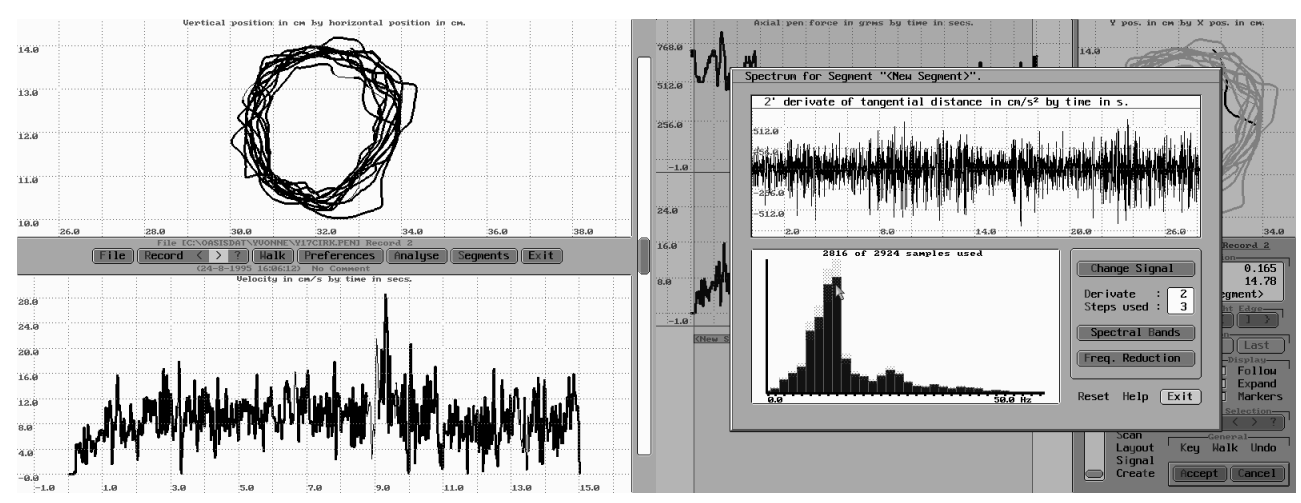

Figure 5: Left panel: example of a graphical display (upper part) of a repeated circular movement made by a patient $(\mathrm{FK})$ with a tremor. Lower part: velocity $(\mathrm{cm} / \mathrm{sec})$ during 15 seconds. Right panel: Power Spectral Density Analysis (PSDA) of the acceleration signal of the same circular movement. The peak frequency (highest bar) lies between 11.9 and $13.5 \mathrm{~Hz}$. 


\section{Discussion}

The results of the present study showed a relationship between bradykinesia, as assessed by means of the SADIMoD ratings, and certain movement variables as measured during the execution of writing and drawing tasks. In the writing task, bradykinesia appeared to be associated with a longer movement time and an increase in the duration of pauses on the paper. Given the wide (normal) variation in handwriting styles and sizes, however, a certain caution is called for when comparing different subjects. For instance, the variation of handwriting size makes it impossible to find a relationship between bradykinesia and micrographia. Yet, the recording of writing movements can be useful in following individual patients during treatment, i.e. enabling the quantification of changes in handwriting due to medication.

In the aiming task, bradykinesia appeared to be associated with a longer initiation period before the start of the movement as well as with a longer pause duration during execution. This finding corresponds with the performance by patients with Parkinson's disease (Marsden, 1989). In the figure-copying task a significant correlation was found only between bradykinesia and movement velocity. A possible explanation for the finding that bradykinesia correlated with initiation time in producing aiming movements (aiming task), but not in copying figures, may be that in this latter task complex (cognitive) processes during actual performance play a greater role than in the first task, which may be planned in advance. The diversity of the research group concerning the severity of the psychiatric conditions would be an important factor in the intersubject differences in cognitive slowing. When in the figure-copying task a distinction was made between the different levels of complexity (familiarity and number of line elements), it appeared that the initiation time in copying simple letters did correlate with bradykinesia, whereas this was not the case in the more complex levels (figures and patterns). This significant association in the copying of simple letters shows that, analogous to the relationship between initiation time and bradykinesia in the aiming task, the severity of bradykinesia is seen most clearly in (cognitive) simple tasks. On the other hand, a study that we conducted in patients with schizophrenia - who were correctly adjusted to their medication and, therefore, showed hardly any movement disorders revealed that initiation time in copying the complex and abstract patterns correlated significantly with the severity of the negative symptoms (established by means of the PANSS) (JogemsKosterman et al., 2001, see the succeeding Chapter). Also Nelson et al. (1990) found that negative symptoms of schizophrenia were particularly associated with cognitive slowing (bradyphrenia) and to a lesser extent with motor slowing.

This study shows that analyses of movements during the performance of writing and drawing tasks enables the quantification of (changes in) the severity of bradykinesia induced by antipsychotic treatment. In principle, the writing tablet may also be applied to quantify a holding tremor, resting tremor and an intention tremor. Although the present study results are promising, especially in view of the fact that the holding tremor can be observed in the performance of continuous graphic tasks, an accelerometer seems to be more useful in this respect. Therefore we have decided to not develop further writing or drawing tasks for measuring tremors.

The study was initially aimed at the measurement of several movement disorders (bradykinesia, tremor(s), rigidity and ataxia). However, within the research population too few 
patients were seen with mild forms of ataxia to assess the applicability of tasks that were developed for this purpose. Because of their handicap (mainly due to co-morbidity), two patients with severe ataxia were not able to participate in the study.

It is not easy to find aspects of performance in writing and drawing tasks that are indicative of the severity of rigidity. Several experiments have been tried in the present study, yet these appeared not to be useful in clinical practice. Moreover, there is a conceptual problem, as all writing and drawing tasks involve planning and active movement, whereas rigidity manifests itself in passive movement. Therefore, for the quantification of rigidity we have decided to search for another instrument.

Both from a scientific and clinical viewpoint, there is a great need for a reliable, and nonaggravating objective instrument. For assessing the severity of side-effects of psychopharmacological agents, the investigator has to rely on the patient's verbal reports and his own subjective impressions during the physical examination. A more fruitful way is to measure the different unwanted clinical symptoms in several dimensions. This strategy has been elaborated in the MASEAS: Multi Axial Side Effect Assessment System (Loonen et al., 1997; Loonen \& Doorschot, 1994). The MASEAS is an instrument in progress, for the assessment of unwanted clinical events (Adverse Clinical Events = ACEs) during research on the efficacy and side-effects of (psycho)pharmacological agents. This instrument assesses at five different levels (axes): (1) complaints of the patient, (2) observable symptoms, (3) results of physical examination, (4) results of chemical examination, (5) results of psychological examination. When there is a combination of certain features at each of some of the mentioned axes, ACEs with a specified severity are defined as clinical syndromes.

The writing and drawing tasks described in this paper will, in a revised form, be part of the MASEAS instrument. Research on the validity and reliability of the instrument is in the stage of preparation.

\section{Acknowledgements}

This study was supported by a grant of the P.V. Petersen Stichting, c/o Lundbeck bv, Amsterdam. We wish to thank Ms. Y.J. Maas for her substantial contribution to the collection of the data and the processing of the results. 


\section{References}

Boshes, B. (1966). Measurement of tremor. Journal of Neurosurgery, 24, 324-330.

Caliguiry, M.P., Bracha, H.S., \& Lohr, J.B. (1989). Asymmetry of neuroleptic-induced rigidity: Development of quantitative methods and clinical correlates. Psychiatry Research, 30, 275-284.

Caliguiry, M.P., Lohr, J.B., Bracha, H.S., \& Jeste, D.V. (1991). Clinical and instrumental assessment of neuroleptic-induced parkinsonism in patients with tardive dyskinesia. Biological Psychiatry, 29, 139-148.

Caliguiry, M.P., Lohr, J.B., \& Jeste, D.V. (1993). Parkinsonism in neuroleptic-naive patients. American Journal of Psychiatry, 150, 1343-1348.

Davis, J.M. (1980). Antipsychotic drugs. In: H.I. Kaplan, A.M. Freedman \& B.J. Sadock (Eds.), Comprehensive textbook of psychiatry (third edition) (pp. 2257-2289). Baltimore: Williams \& Wilkins.

De Jong, W.P., Hulstijn, W., Jogems-Kosterman, B.J.M., \& Smits-Engelsman, B.C.M (1996). OASIS software and its application in experimental handwriting research. In: M.L. Simner, C.G. Leedham, \& A.J.W.M. Thomassen (Eds.), Handwriting and drawing research: Basic and applied issues (pp. 429440). Amsterdam: IOS Press.

Guy, W. (1976). ECDUE assessment manual for psychopharmacology. Rockville: National Institute of Mental Health.

Haase, H.J. (1977). Therapie mit Psychopharmaka und anderen seelisches Befinden beeinflussenden Medikamenten. Stuttgart: FK Schattauer Verlag.

Jogems-Kosterman, B.J.M., Zitman, F.G., Van Hoof, J.J.M., \& Hulstijn W. (2001). Psychomotor slowing and planning deficits in schizophrenia. Schizophrenia Research, 48, 317-333.

Loonen, A.J.M., \& Zwanikken, G.J. (1987). Farmaca, psychofarmaca, klinisch onderzoek. 'sHertogenbosch: WULCO.

Loonen, A.J.M., \& Doorschot, C.H (1994). Schaal voor het vastleggen van door geneesmiddelen veroorzaakte bewegingsstoornissen (SADIMoD). Vught: Psychiatrisch Ziekenhuis Reinier van Arkel.

Loonen, A.J.M., \& Doorschot, C.H (1996). Motorische bijwerkingen bij antipsychoticumgebruik. COBO Bulletin, 29 (Sept), 19-25.

Loonen, A.J.M., Doorschot, C.H., Jogems-Kosterman, B.J.M., Van Hoof, J.J.M., Hulstijn, W., \& Zitman, F.G. (1997). Nieuw instrumentarium voor het meten van bijwerkingen. COBO Bulletin, 30 (Jan), 31-40.

Loonen, A.J.M., \& Doorschot, C.H. (1994). Klinisch veiligheidsonderzoek van geneesmiddelen: Structuur van een documentatiesysteem. Ziekenhuisfarmacie, 10, 41-44.

Marsden, C.D. (1989). Slowness of movement in Parkinson's disease. Movement Disorders, 4 (Suppl 1), S26-S37.

Martinez, N (1966). Measurement of rigidity with a strain gauge myokinetograph. Journal of Neurosurgery, 24, 315-316.

Nashold, B.S. (1966). Measurement of tremor. Journal of Neurosurgery, 24, 320-323.

Nelson H.E., Pantelis, C., Carruthers, K., Spellers, J., Baxendale, S., \& Barnes, T.R. (1990). Cognitive functioning and symptomatology in chronic schizophrenia. Psychological Medicine, 20, 357-365.

Sabbe, B.G.C., Hulstijn, W., Van Hoof, J.J.M., \& Zitman, F.G. (1996). Fine motor retardation and depression. Journal of Psychiatric Research, 30, 295-306.

Simpson G.M., \& Angus J.W.S. (1970). A rating scale for extrapyramidal side effects. Acta Psychiatrica Scandinavica, 212 (suppl), 11-19. 
Antipsychotics, movement disorders and fine motor performance | 53

Teräväinen, H., Tsui, J.K.C., Mak, E., \& Calne, D.B. (1989). Optimal indices for testing parkinsonism rigidity. Canadian Journal of Neurological Science, 16, 180-183.

Van Hoof, J.J.M., Sabbe, B.G.C., Hulstijn, W., Van Mier, H., \& Jogems-Kosterman, B.J.M. (1996). Het kopiëren van figuren en psychomotorische vertraging bij depressie. Tijdschrift voor Psychiatrie, 38, 52031.

Van Hoof, J.J.M., Jogems-Kosterman, B.J.M., Sabbe, B.G.C., Zitman, F.G., \& Hulstijn W. (1998). Differentiation of cognitive and motor slowing in the Digit Substitution Test (DST): Differences between depression and schizophrenia. Journal of Psychiatric Research, 32, 99-103.

Webster, D.D. (1966). Rigidity in extrapyramidal disease. Journal of Neurosurgery, 24, 299-307. 


\title{
Chapter 4
}

\section{Psychomotor slowing and planning deficits in schizophrenia ${ }^{1}$}

\begin{abstract}
In the present study the relative contribution of cognitive and motor processing to psychomotor slowing in schizophrenia was investigated using three tasks: a simple linecopying task and a more complex figure-copying task, both following a reaction paradigm, and a standard psychomotor test, the Digit Substitution Test (DST). Various movement variables of the task performances were derived from recordings made with the aid of a digitizing tablet. The patients with schizophrenia appeared to be about one third slower in their total performance time on all three tasks when compared to healthy controls, which suggests a general psychomotor slowing in this group. When itemized over the various movement variables, this slowing was found in both initiation time and movement time in the copying tasks and in the DST in the time to match the symbol and the digit, but not in writing the digit. Furthermore, in the figure-copying task it was found that increased figure complexity or decreased familiarity prolonged initiation time, but these latency increases were not significantly larger for the schizophrenia group as a whole, but only for a subgroup of patients with higher scores on negative symptoms. Regarding reinspection time the effects of familiarity were larger in the schizophrenia group as a whole. These group findings suggest that patients tend to plan their actions less in advance, which, in the case of the more complex or unfamiliar task conditions, is a less sophisticated planning strategy. Given the longer latencies in patients with more severe negative symptoms, it seems that these patients have problems with turning a plan into action. The present study provides evidence of psychomotor slowing and planning deficits in schizophrenia.
\end{abstract}

\footnotetext{
${ }^{1}$ Based on:
}

Jogems-Kosterman, B.J.M., Zitman, F.G., Van Hoof, J.J.M., \& Hulstijn, W. (2001). Schizophrenia Research, $48,317-333$. 


\section{Introduction}

There is substantial evidence from both clinical and neuropsychological research that schizophrenia is associated with psychomotor slowing in which the term 'psychomotor' is used to characterize the wide variety of human actions involving both cognitive and sensorimotor processes. Although this slowing in the initiation and execution of action is not restricted to schizophrenia and can also be observed in other patient groups (Benson, 1990; Bermanzohn \& Siris, 1992), it is viewed as an important characteristic of the disease, and categorized as one of the main negative symptoms of schizophrenia (Crow, 1980) and the 'psychomotor poverty' syndrome (Liddle, 1987). Clinical observations are supported by many studies showing that patients with schizophrenia perform poorly on almost every neuropsychological and cognitive test, as well as on tests assessing psychomotor speed (e.g., Shapiro \& Nelson, 1955; Wolkin et al., 1992; Jeste et al., 1996; Brébion et al., 1998; for a further review, see Goldberg \& Gold, 1995).

It has been suggested that these poor test performances may be the result of a more basic psychomotor slowing or deficit (Goldberg \& Gold, 1995; Gourovitch \& Goldberg, 1996). Brébion et al. (1998) found that memory impairments in a group of patients with schizophrenia could at least partially be explained by a reduced processing speed. According to King (1991), patients with schizophrenia suffer from a fundamentally impaired integration of sensory and motor processes, which hinders the learning and execution of more complex tasks. However, patients with schizophrenia also appear to exhibit significant problems with the planning and organization of action, which suggests the involvement of disturbances in higher cognitive, executive processing (Widlöcher \& Hardy-Bayle, 1989; Frith, 1992, 1995). Nelson et al. (1990) indeed found that in patients with schizophrenia cognitive speed was more affected than motor speed. More specific planning deficits in schizophrenia have been found using explicit tasks for planning ability, such as the Tower of London (Shallice, 1982; Morris et al., 1995; Hanes et al., 1996; Pantelis et al., 1997), a task in which a set of balls have to be re-ordered so that their positions match a certain goal arrangement.

The actual study of the relative contribution of cognitive and motor processing to psychomotor slowing is complicated by the fact that these processes can hardly be separated in daily activities and in most neuropsychological tests. The use of reaction time tasks (see King, 1991) has been advocated as a solution. For example, Malla et al. (1995) tried to separate planning (reaction time) and execution (movement time) in an aiming task. The feasibility of such a separation is based on the assumption that the cognitive processes involved in the planning of the movement take place during the reaction time interval, whereas the motor processes mainly occur during movement time. However, this distinction may be too artificial, since cognitive processes, necessary for further planning or correction of movements, may also be engaged during the execution phase. Particularly in complex tasks the required sequence of movements can hardly be planned entirely in advance. It has been found that increased task complexity in a computerized version of the Tower of London (Pantelis et al., 1997) prolonged the duration of the execution period more strongly in patients with schizophrenia than in healthy subjects, which suggests that the patients needed more time during the task for further planning and monitoring of their movements. 
In the present study the relative contribution of cognitive and motor processing to psychomotor slowing is investigated by analyzing the performance on two copying tasks, a simple line-copying task and a more complex figure-copying task. These tasks follow a reaction time paradigm in that the stimuli, which are presented on a computer screen, have to be copied as fast as possible on a digitizing tablet with an electronic pen. The employment of this equipment allows accurate measurements of various aspects of the drawing movements produced during these psychomotor tasks. This technique not only allows the determination of the interval before a movement is started (reaction or initiation time) and the time needed to execute movements (movement time), but also the calculation of movement variables such as mean velocity, distance covered by the pen, duration of pauses during drawing as well as performance characteristics of the movement such as straightness and fluency of lines (Hulstijn, 1996; De Jong et al. 1996). In the more complex figure-copying task, subjects can reinspect the stimulus (which disappears again at the first drawing movement) when they really do not know how to complete the drawing. This variable is called reinspection time.

In earlier studies this recording technique was used to demonstrate both cognitive and motor slowing in patients with a major depression by analyzing these movement variables (Van Hoof et al., 1993, 1998 and Chapter 2 of this thesis; Sabbe et al. 1996a, 1996b, 1997, 1998). In the present study the variables are measured to characterize psychomotor slowing of patients with schizophrenia. As most neuropsychological tests, figure-copying tasks involve several cognitive, sensory and motor processes (Van Sommers, 1989; Van Galen, 1991; Hulstijn, 1994). First, perceptual processes are employed for the visual analysis of the stimulus, after which features and/or global information of the stimulus can be stored in a temporary (episodic) store. If the stimulus is or resembles a familiar object, a visual representation may be retrieved from long- term visual memory and/or a name may be retrieved from long-term verbal memory. Secondly, visualspatial processes are applied to assure adequate perception and planning of the orientation and relative size of lines, particularly if the stimulus is a novel or unfamiliar pattern. Next, the movement sequence must be planned, which does not mean that the planning of the stroke order of the entire figure has to be completed before the first movement is initiated, but at least the first element or the point and direction of the first movement should have been chosen in advance. For the sake of convenience, in the remaining part of this paper we will refer to the above-mentioned, more cognitive processes with the term planning, which thus includes all preparatory processes (perception, storage and visual-spatial processing) that are involved. These processes will take up by far the largest part of the initiation interval. The other processes involved in figure copying may be denoted by the term motor. These motor processes comprise the programming, coordination, initiation and execution of the muscle commands needed for drawing, as well as the monitoring of visual feedback to correct errors. Motor processes consume only a small part of the initiation time and pause duration during execution and mainly take place during movement time. Hence, problems with motor control or a slowing of movements will have large effects on movement durations, although the planning and programming of the succeeding movements may not be restricted to the pauses and pen-up periods, but may affect movement execution as well. 
In the line-copying task, single lines had one of four possible directions; copying them resembles the more traditional four-choice reaction task. In the more complex-copying task planning demands (which we take to include memory and visual-spatial processing) are manipulated by varying both the number of line elements of the figures and their familiarity. This task was originally designed to measure the time it takes to plan a movement sequence (Van Mier \& Hulstijn, 1993). Therefore the length of the sequence was systematically varied by using stimuli for copying consisting of either four or eight lines. A second variable that influences the time to plan a sequence is the familiarity or the amount of practice with the order in which the lines are drawn. In the simplest condition this order can be retrieved from memory, as in letters in which the order of the strokes is automatized. Further, figures that can be recognized and verbally labeled are more easily kept in a temporary store than completely unfamiliar and novel patterns. Therefore, to manipulate these memory variables, three categories of stimuli were used in the figure-copying task: well-learned letters, familiar figures and unfamiliar patterns. Impairments in planning (including problems with memory and visual-spatial processes) would be likely to impact initiation time, reinspection time and duration of pauses during execution. Motor deficits would be particularly reflected in movement time and movement velocity.

Although these figure-copying tasks have been used successfully in research on psychomotor slowing in depression (Van Hoof et al., 1993; Sabbe et al., 1996a, 1996b, 1997, 1998), they have not been used in the study of schizophrenia. This is why a standard test for psychomotor speed, the Digit Substitution Test (DST), which is part of the Wechsler Adult Intelligence Scale (WAIS) (Wechsler, 1956), is included in the present study. By administering this test on a digitizer and employing the same recording technique it is possible to separately measure for each symbol the time needed to find the corresponding digit (matching time) and the time spent on writing that digit (writing time), which is an attempt to tease out cognitive and motor processes.

To sum up, the following hypotheses were tested.

First, if it is true that patients with schizophrenia suffer from a rather basic sensorimotor or psychomotor deficit (King, 1991; Goldberg \& Gold, 1995), one would expect these patients to show slowing even in the simple line-copying task. In addition, it might be expected that this slowing will not be disproportionally increased in their performance on the more complex-copying task.

Second, if the cognitive contribution to psychomotor slowing is more powerful than its motor component (Nelson et al., 1990), patients with schizophrenia will show a greater degree of slowing on those movement variables which reflect cognitive processing more strongly. The duration of these cognitive processes reflect the ease with which the stimulus is visually encoded and put into a temporary store, and the ability to plan a sequence of movements before and during drawing. More specifically, in line copying, but particularly in figure copying, initiation time, reinspection time and pause duration in execution will be more strongly affected than movement time or movement velocity. For the DST, a longer matching time is predicted for patients with schizophrenia, but no group difference in the time needed to write the digits (Van Hoof et al., 1998, see Chapter 2).

Third, if problems in the planning of movements are prominent in schizophrenia (Widlöcher \& Hardy-Bayle, 1989; Frith, 1992, 1995; Morris et al., 1995; Hanes et al., 1996; Pantelis et al., 
1997), one would expect the effects of number of lines and figure unfamiliarity in the complexcopying task to be greater in patients with schizophrenia. These effects will, again, be expected on those variables reflecting primarily cognitive processing, such as initiation time, reinspection and pause duration in execution.

The three hypotheses given above are formulated for the group of schizophrenic subjects as a whole. However, since psychomotor slowness is categorized as a negative symptom, one might expect this slowing to correlate with severity of negative symptoms (Liddle \& Morris, 1991; Nelson et al., 1990). The fourth prediction therefore is that the hypothesized group differences will hold stronger for patients with clear negative symptoms.

\section{Method}

\section{Subjects}

Subjects included 19 patients (15 male; four female) suffering from schizophrenia (DSM-III-R, American Psychiatric Association, 1987) and 19 healthy controls matched for age, sex and educational level. The mean ages of the patient and the control group were 37.0 years $(\mathrm{SD}=6.9$; range, 21-47 years) and 37.5 years ( $\mathrm{SD}=7.4$; range, 23-49 years), respectively. Educational level was scored on a 7-point scale, ranging from 'less than primary school' to 'university'. The median score in both groups was 6: 'high school/college'.

Patients were treated at the Psychiatric Center of Nijmegen, the Netherlands $(n=17)$ or at the Department of Psychiatry of the University Hospital of Nijmegen $(n=2) ; 14$ patients were outpatients, five patients were hospitalized at the moment of testing. In order to reduce intervening effects the patients were selected on the following criteria: (1) they were in a stable phase of their disease (i.e. showing no clear florid symptoms), (2) they were only taking one classical antipsychotic drug (mean dosage in haldol equivalents was 7.6; $\mathrm{SD}=5.4$; range, 1-20) and no other concomitant medication, (3) they had no severe neurological or physical illness that could influence the fine motor function of hands and arms, and (4) they were not diagnosed with other severe psychiatric disorders. Patients were usually tested after a short visit to their own doctor; they did not show any signs of alcohol or drug abuse at the time of testing. Prior to participating in the study patients gave their informed consent.

Our controls were paid volunteers recruited by local advertising. They were asked to fill out a questionnaire in order to exclude individuals using psychopharmacological medication and/or other addictive drugs, or individuals with a neurological, physical or psychiatric disease.

\section{Clinical assessment}

For the patient group schizophrenic symptoms were assessed using the Positive and Negative Syndrome Scale (PANSS; Kay, Fiszbein \& Opler, 1987). In both patients and controls the prevalence of extrapyramidal motor symptoms was evaluated by means of the ExtraPyramidal Symptoms Scale (EPS; Simpson \& Angus, 1970) and the Abnormal Involuntary Movement Scale 
(AIMS; Guy, 1976). Both patients and controls completed the Self-rating Depression Scale (SDS; Zung, 1965) to assess depressive complaints.

\section{Apparatus, tasks and procedure}

While performing the writing and drawing tasks the subjects were seated in a quiet room. Writing and drawing movements were recorded by means of a personal computer, a Calcomp 2500 digitizer, and a special pressure-sensitive pen (Teulings \& Maarse, 1984). To familiarize the subjects with the apparatus at the beginning of the session they performed two writing tasks, the first consisting of copying a Dutch sentence ten times and the second involving the production of repetitive and/or alternating writing movements with the letters ' $e$ ', ' $\mathrm{l}$ ' and ' $\mathrm{m}$ '.

\section{Copying tasks}

Two copying tasks were used in this study. In the first task simple lines had to be copied. These lines had one of four orientations: vertical, horizontal, oblique to the right and oblique to the left. The second, more complex copying task consisted of 24 stimuli divided into three 'familiarity' categories: letters, familiar figures and unfamiliar patterns. Each category was divided into two 'complexity' groups consisting of stimuli of either four or eight strokes (for examples see Figure 1).

The stimuli of the two copying tasks were presented on a computer screen that was placed in front of the subject. The four line directions of task one were repeated six times in a random order, resulting in a total of 24 stimuli. The 24 stimuli of the second task were presented in two sets of 12 stimuli. Each stimulus had to be copied within the confines of one of five boxes $(40 \times 50 \mathrm{~mm})$ indicated on the digitizer surface, starting with the leftmost box. Drawing took place on a normal sheet of paper (A4 format) that was put on the digitizer with the boxes still clearly visible. After the fifth box had been filled the sheet of paper was moved upward to allow the subject to copy the next figures on a blank area of the sheet. The subjects were instructed to hold the pen a little above the center of the boxes. After the pen had been held in this position for $15 \mathrm{~ms}$, a warning signal of 500 ms was given. After another $50 \mathrm{~ms}$ the stimulus was presented. The subjects had to copy the stimulus as fast and as accurately as possible within the confines of the box. As soon as the subject started drawing the stimulus disappeared from the screen. This was done to prevent the subjects from copying the figures line by line instead of planning the sequence as a whole in advance (Van Mier et al., 1993; Van Hoof et al., 1993; Sabbe et al., 1996b). In the figure-copying task the subjects had the opportunity to reinspect the stimulus. They were, however, asked to use this option only when they really did not know how to complete the drawing without making errors or omissions. In order to reinspect the stimulus the subjects had to move the pen tip towards and touch a red square of 20 by $20 \mathrm{~mm}$ that was marked on the digitizer. As soon as the subject restarted drawing the stimulus disappeared again. 


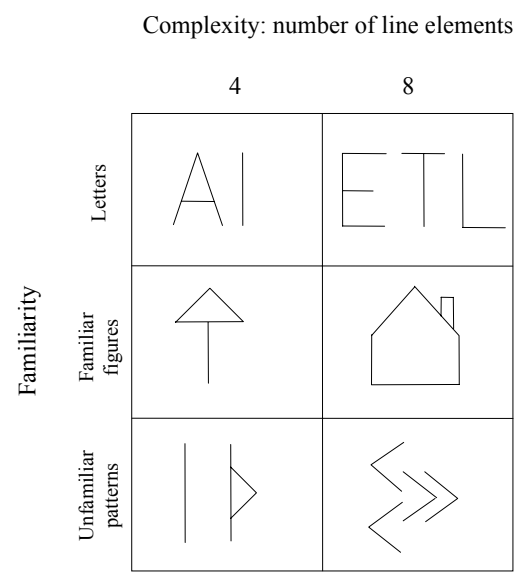

Figure 1: Examples of stimuli used in the figure-copying task.

DST

As in a previous study (Van Hoof et al., 1998), one of the tests we used here was the Dutch version of the Symbol Digit Substitution Test, which is a subtest of the Wechsler Adult Intelligence Scale (WAIS) (Wechsler, 1956). In this version a series of symbols has to be substituted by digits as fast as possible during 90 seconds by using a key consisting of nine symbol-digit pairs. In the English version symbols have to be filled in instead of digits. The advantage (Lezak, 1983) of the Dutch revised version is that it elicits the more familiar response of number writing. In this study the standard form used for the DST was placed on the digitizer.

\section{Recording and analysis}

The $\mathrm{x}$ and $\mathrm{y}$ coordinates of the pen tip on and up to $5 \mathrm{~mm}$ above the digitizer were sampled with a frequency of $100 \mathrm{~Hz}$ and a spatial accuracy of $0.2 \mathrm{~mm}$. Signals were filtered by means of a FastFourier analysis (Teulings \& Maarse, 1984). The movement trajectories within each trial were automatically segmented on velocity minima. Based on pen pressure (threshold: $0.24 \mathrm{~N}$ ) these segments were defined as either pen-up periods or pen-down periods. The pen-down periods during which the movement velocity was below $20 \%$ of the mean velocity were defined as pause periods; the remaining periods were taken to represent movement periods.

For the copying tasks the following variables were calculated for each individual line or figure. The initiation time was operationalized as the pen-up period between the appearance of the stimulus on the computer screen and the first pen-down period. The total execution time was divided into movement periods, pause periods, and pen-up periods. In addition, the mean velocity during the movement periods was calculated as well as the total length of the trajectory covered by 


\section{2 | Chapter 4}

the pen on the paper (distance). Calculations for the figure-copying task also included the reinspection time operationalized as all pen-down and pen-up periods from the moment the pen pause started, the time during which the pen was moved from the drawing box to the stimulus reinspection box up and until the moment when the drawing movement was resumed. Further, the drawings produced in the figure-copying task were evaluated by two independent raters on two types of errors, i.e. errors which had not led to a severe distortion of the copy of the stimulus figure and errors which had distorted the drawing beyond recognition (for a more detailed description of this scoring method, see Sabbe et al., 1996b).

For the DST calculations consisted of: raw score, i.e. the number of correct digits produced within 90 seconds; matching time (comparable to initiation time), operationalized as the accumulated pen-up periods between two successive digits and the pause intervals at the end and at the beginning of these digits; writing time (comparable to execution time) operationalized as the time between the start of the first and completion of the final movement within a digit; mean velocity, matching time, writing time, as well as distance were calculated as a mean value per digit. In the latter calculations the first digit on each new line was not taken into account because of the large distance that had to be covered by the pen.

Statistical evaluation (using SPSS for Windows) was performed with analysis of variance (ANOVA) for each movement variable with one between-subject factor, viz. Group (patients, controls). For the figure-copying task a two-way ANOVA for repeated measurements was used with two within-subject factors: Familiarity (letters, familiar figures, unfamiliar patterns) and Complexity (four strokes, eight strokes). The mean scores on the clinical rating scales were subjected to a Mann-Whitney U test. For the patient group, correlations between clinical rating scores and movement variables were calculated by means of Spearman's $\rho$.

\section{Results}

\section{Clinical ratings}

Table 1 presents the scores on the clinical rating scales. When compared to the maximum scores that can be obtained on each subscale of the PANSS (49 for both positive and negative symptoms; 112 for general psychopathology), the mean scores of our patient group were rather low. The mean EPS score for the patients was 2.4 , which is very low when compared to the maximum achievable score of 40; only five patients (26\%) obtained a rating of 2 ('moderate') on one or more items. For the AIMS we took the sum of the first seven items which record the severity of abnormal movements (range: 0-4) in seven areas of the body. Following the criterion for dyskinesia as proposed by Smith et al. (1979), i.e. a rating of 3 (moderate) or more in at least one body area, only five of our patients $(26 \%)$ showed features of dyskinesia. Despite the fact that patients had low scores on the EPS and AIMS, these scores were nevertheless significantly higher than those of the controls (see Table 1). 
Table 1: Mean values and standard deviations (in brackets) for the clinical rating scores for patients and controls. Statistical analysis: Mann-Whitney U Test.

\begin{tabular}{|c|c|c|c|c|c|c|}
\hline & & \multicolumn{2}{|c|}{ Patients } & \multicolumn{2}{|c|}{ Controls } & Statistics \\
\hline \multicolumn{6}{|c|}{ PANSS } & \\
\hline & Positive symptoms & 11.26 & $(2.86)$ & - & & - \\
\hline & Negative symptoms & 14.21 & $(4.96)$ & - & & - \\
\hline & General psychopathology & 28.84 & $(5.43)$ & - & & - \\
\hline \multicolumn{2}{|l|}{ EPS } & 2.42 & $(3.06)$ & 0 & $(0)$ & $\mathrm{U}=66.5, \mathrm{p}<0.001$ \\
\hline \multicolumn{2}{|c|}{ AIMS/item 1-7 } & 3.37 & $(0.97)$ & 0.05 & $(0.23)$ & $\mathrm{U}=31.5, \mathrm{p}<0.001$ \\
\hline \multicolumn{2}{|l|}{ SDS } & 37.21 & $(8.85)$ & 28.68 & $(5.28)$ & $\mathrm{U}=76.0, \mathrm{p}<0.002$ \\
\hline
\end{tabular}

On the SDS the cut-off total score for depressive disorder is 50; total scores can range between 20 and 80 (Zung, 1965). Two patients (10.5\%) had a score above 50. The mean total SDS score in the patient group was significantly higher than in the control group (see Table 1).

\section{Copying tasks}

The mean values of the movement variables (initiation time, movement time, movement velocity and distance) for the copying tasks for patients and controls are displayed in Figure 2. The results for these variables were highly comparable: in both tasks patients showed a significantly longer initiation time and movement time as compared to the controls, whereas no significant group differences were found for movement velocity. Although the patients showed longer movement trajectories (distance) in both copying tasks as compared to the controls, this difference only approached significance $(\mathrm{p}=0.06)$.

Furthermore, in the figure-copying task, which makes higher demands on cognitive processing than the line-copying task, the periods in which the pen was above the paper (pen-up time) proved to be significantly $(\mathrm{p}<0.04)$ longer for patients than for controls (see Table 3 ). The duration of the reinspection intervals in the figure-copying task was also significantly $(p<0.05)$ longer for patients as compared to the controls. No significant difference was found in the number of reinspections $(\mathrm{F}(1,37)=1.37, \mathrm{p}=0.25)$ between the two groups. Pause durations during line copying were negligible in both patients (mean: $6 \mathrm{~ms}$ ) and controls (mean: $8 \mathrm{~ms}$ ), and although they were longer in the figure-copying task (283 ms and $223 \mathrm{~ms}$, respectively), no significant group difference $(p=0.40)$ was found. No significant group differences were found in the number and type of errors in the figure-copying task. 

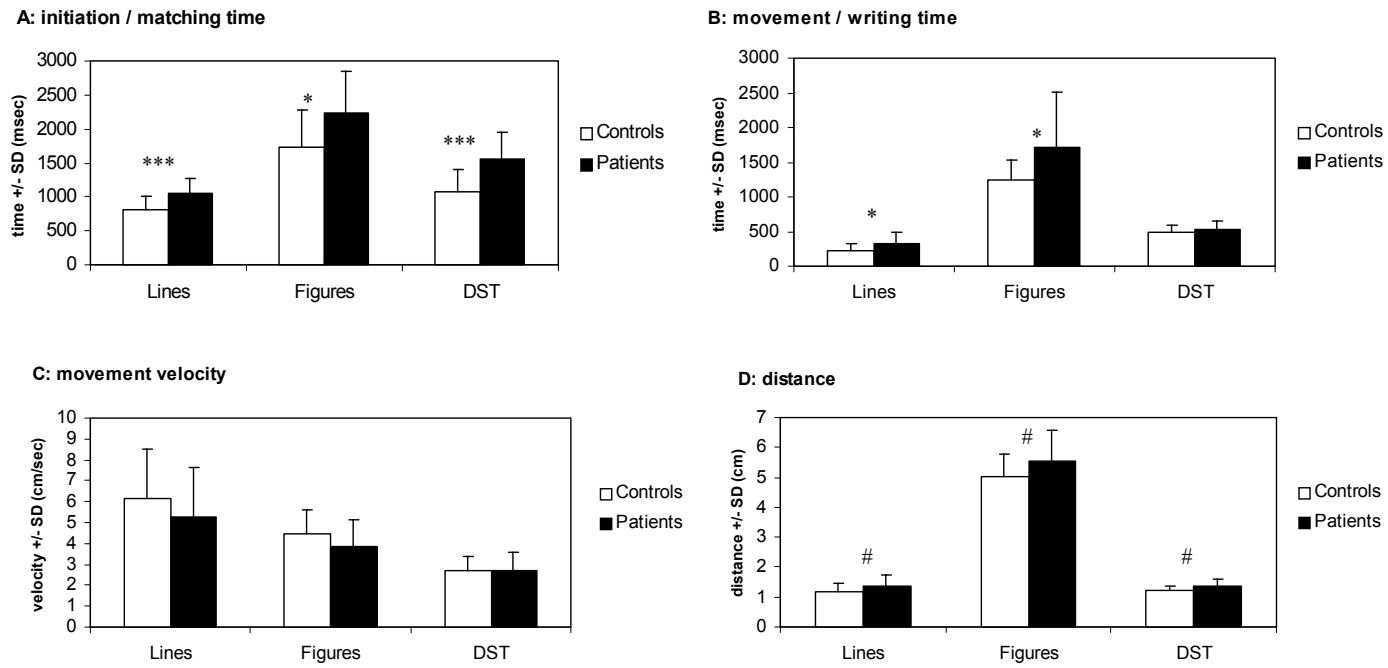

Figure 2: Mean values and standard deviations for initiation time and matching time (A), movement time and writing time (B), movement velocity (C) and distance (D) for the line- copying task ('Lines'), the figurecopying task ('Figures') and the DST. *** $\mathrm{p}<0.001,{ }^{* *} \mathrm{p}<0.01,{ }^{*} \mathrm{p}<0.05, \# \mathrm{p}<0.10$

If patients with schizophrenia show planning problems (which we take to include possible problems with the encoding and storage of the stimulus) one would expect them to be more challenged by the manipulations in the figure-copying task than the controls. These manipulations the degree of unfamiliarity of the figures and the variation of complexity- had significant effects on all movement variables when averaged over both patients and controls (see Table 2). However, the main effects of Complexity on movement time, pen-up time and distance may not be of importance since they are simply due to the doubling of the number of strokes. Significant main effects of Complexity and Familiarity were also found for movement velocity, which means that subjects seem to slow down when task requirements increase.

Interaction effects were calculated to find out whether these manipulations had affected patients' performance more than the performance of the controls. No significant interactions were found for initiation time (see also Figure 3A) between Group and Familiarity or between Group and Complexity. On the other hand, when figures became more unfamiliar patients reinspected the stimuli significantly longer when compared to the controls. Patients appeared to encounter difficulties particularly with the most complex and unfamiliar patterns (see Figure 3C). The interaction between Group and Complexity, however, failed to reach significance for reinspection time $(p=0.10)$. 
Table 2: F-values of the main and interaction effects of Group (G), Familiarity (F) and Complexity (C) on the movement variables of the figure-copying task.

\begin{tabular}{|c|c|c|c|c|c|c|}
\hline & \multicolumn{3}{|c|}{ Main effects } & \multicolumn{3}{|c|}{ Interaction effects } \\
\hline & $\begin{array}{l}\text { Group } \\
\text { (G) }\end{array}$ & $\begin{array}{l}\text { Familiarity } \\
\text { (F) }\end{array}$ & $\begin{array}{l}\text { Complexity } \\
\text { (C) }\end{array}$ & G X F & $\mathrm{G} \times \mathrm{C}$ & G X F X C \\
\hline & $F(1,36)$ & $F(2,35)$ & $F(1,36)$ & $\mathrm{F}(2,35)$ & $F(1,36)$ & $\mathrm{F}(2,35)$ \\
\hline Initiation time & $6.89 *$ & $23.95 * * *$ & $65.52 * * *$ & 0.85 & 2.59 & 0.69 \\
\hline Movement time & $6.20^{*}$ & $43.73 * * *$ & $340.61 * * *$ & 2.05 & $5.65^{*}$ & 1.64 \\
\hline Pen-up time & $4.88^{*}$ & $83.12 * * *$ & $372.02 * * *$ & 0.40 & 2.14 & 0.06 \\
\hline Pausing time & 0.72 & $27.43 * * *$ & $28.54 * * *$ & 0.96 & 1.29 & 1.86 \\
\hline $\begin{array}{l}\text { Reinspection } \\
\text { time }^{1}\end{array}$ & $4.33^{*}$ & $24.63 * * *$ & $57.67 * * *$ & $4.24 *$ & $2.86^{\#}$ & $2.92^{\#}$ \\
\hline $\begin{array}{l}\text { Movement } \\
\text { velocity }\end{array}$ & 2.39 & $21.76 * * *$ & $93.70 * * *$ & 0.79 & 2.77 & $5.78 * *$ \\
\hline Distance & $3.70^{\#}$ & $45.33 * * *$ & $521.67 * * *$ & 0.77 & 1.74 & 1.62 \\
\hline
\end{tabular}

Examination of the execution period revealed that the interaction between Group and Complexity was significant for movement time (see also Figure 3B), although this is hardly surprising since, when slowing is present in drawing four lines, it may be expected to be twice as high in the case of eight lines. The effects of Familiarity and Complexity on pause time, pen-up time, and movement velocity were not significantly different for patients and controls (see Table 2). The higher order interaction between Group, Familiarity and Complexity was only significant for movement velocity. It appeared that the above-mentioned main effect of Complexity on movement velocity was less pronounced for patients in the case of letters. The cause of this is not clear.

$D S T$

The standard test score on the DST, the raw score, was significantly $(\mathrm{F}(1,35)=14.83, \mathrm{p}<0.001)$ lower in the patients (44.2) when compared to the controls (58.5). The mean values of matching time, writing time, movement velocity and distance are shown in Figure 2. The patients had a significantly longer matching time $(p<0.001)$ as compared to the controls. No significant differences in writing time $(p=0.23)$ and movement velocity $(p=0.85)$ were found between the two groups in this test. Although it was not significant, patients tended to write the digits bigger than their controls. 
66 | Chapter 4

A: initiation time

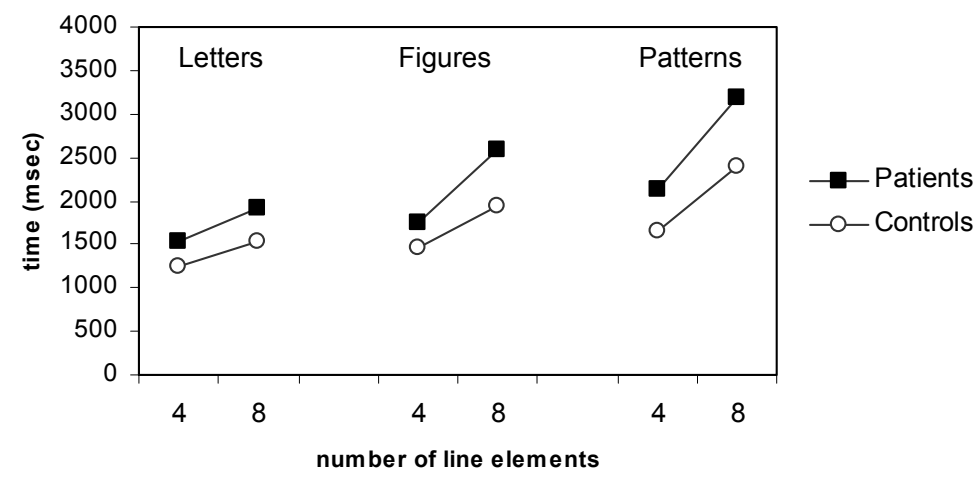

B: movement time

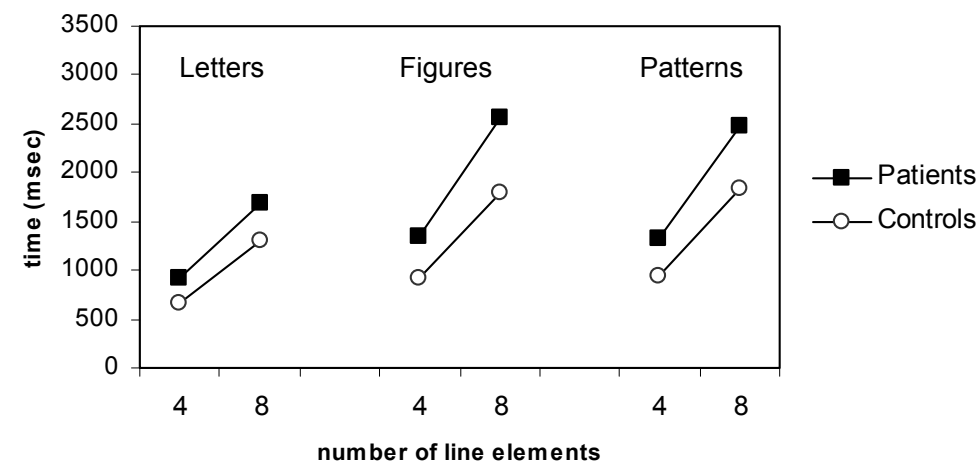

C: reinspection time

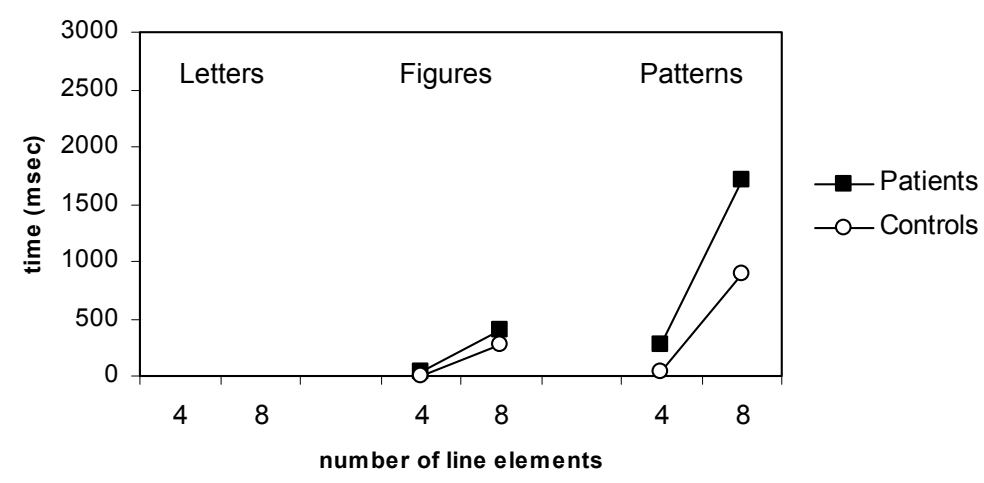

Figure 3: For patients and controls the mean values are given of initiation time (A), movement time (B) and reinspection time (C) as a function of Familiarity (letters, figures and patterns) and Complexity (four line elements, eight line elements). 
Table 3. Differences between patients and controls expressed as percentages of the means of the control group, and their corresponding statistics.

\begin{tabular}{|c|c|c|c|c|c|c|}
\hline & \multicolumn{2}{|c|}{ Line-copying task } & \multicolumn{2}{|c|}{ Figure-copying task } & \multicolumn{2}{|l|}{ DST } \\
\hline & $\begin{array}{l}\text { Relative } \\
\text { difference (\%) }\end{array}$ & $\mathrm{F}(1,37)$ & $\begin{array}{l}\text { Relative } \\
\text { difference }(\%)\end{array}$ & $\begin{array}{l}\mathrm{F} \\
(1,37)\end{array}$ & $\begin{array}{l}\text { Relative } \\
\text { difference }(\%)\end{array}$ & $\mathrm{F}(1,35)$ \\
\hline Total time & 32.4 & $13.24 * * *$ & 32.5 & $8.66^{* *}$ & 33.8 & $14.91^{* * *}$ \\
\hline \multicolumn{7}{|l|}{ Initiation } \\
\hline initiation time / & 29.6 & $13.01 * * *$ & 29.7 & $6.89 *$ & & \\
\hline matching time & & & & & 44.4 & $16.51 * * *$ \\
\hline \multicolumn{7}{|l|}{ Execution } \\
\hline movement time / & 43.5 & $4.87^{*}$ & 38.7 & $6.20 *$ & & \\
\hline writing time & & & & & 10.4 & 1.47 \\
\hline pen-up time & & & 22.5 & $4.88^{*}$ & & \\
\hline pausing time & & & 21.7 & 0.72 & & \\
\hline reinspection time & & & 100.0 & $4.33 *$ & & \\
\hline movement velocity & -13.4 & 1.12 & -13.9 & 2.39 & 1.9 & 0.04 \\
\hline distance & 16.0 & $3.76^{\#}$ & 10.6 & $3.70^{\#}$ & 11.7 & $3.65^{\#}$ \\
\hline
\end{tabular}

\section{Comparison of movement variables in the three tasks}

To test the hypothesis of a rather basic psychomotor slowing in the patient group, we compared the total performance durations on the three tasks (c.f. King, 1991; Goldberg \& Gold, 1995). For copying one single line (i.e. initiation and execution) in the line-copying task, patients needed on average $1380 \mathrm{~ms}$ against $1040 \mathrm{~ms}$ in the control group ( $<<0.001)$. In the figure-copying task these values were $5950 \mathrm{~ms}$ for patients and $4490 \mathrm{~ms}$ for controls $(\mathrm{p}<0.01)$. When calculating the slowing in the patient group as a proportion of the means of the controls (see Table 3), we found that patients were about $32 \%$ slower than the controls on both tasks. Also in figure copying, although this task was more specifically designed to measure the effects of task complexity, the relative differences between patients and controls were similar: $32 \%$ for letters, $30 \%$ for figures and $35 \%$ for patterns. On the DST, patients needed on average $2090 \mathrm{~ms}$ for the matching and writing of one single digit against $1560 \mathrm{~ms}$ in the controls $(\mathrm{p}<0.001)$, which also means a slowing of about $34 \%$ in the patient group as compared to the control group.

Table 3 also presents the relative differences between patients and controls for the various movement variables. This comparison reveals that, proportionally, the performance difference between patients and controls was similar in the two copying tasks with respect to initiation time and movement time: in both tasks patients were about 30\% (initiation time) and $40 \%$ (movement time) slower than the controls. Group differences in matching time were somewhat larger than those in initiation time. On the other hand, there were no significant differences in writing time, which is in contrast with the differences found in movement time in the copying tasks. Table 3 further shows 


\section{8 | Chapter 4}

that in all three tasks the prolonged writing or movement time in the patient group was to a greater extent due to a longer distance rather than to a lower movement velocity.

\section{Relationship with negative symptoms}

To investigate the relationship between the movement variables and the severity of the negative symptoms, correlations were calculated (see Table 4). It was expected that slowing in task performance would be more prominent in patients with clear negative symptoms. The scores on negative symptoms appeared to correlate most strongly with initiation time in the figure-copying task. Other close correlations were between negative symptoms and movement velocity in line- and figure-copying tasks and pause durations in figure copying.

Further examination of the correlation between negative symptoms and initiation time in the figure-copying task revealed that the most complex figures and patterns were mainly responsible for this relationship. This is visualized in figure $4 \mathrm{~A}$ which shows the mean values of the initiation time for the two subgroups of patients, i.e. patients with either low negative symptom scores (one or two 'moderate' scores out of seven) or high negative symptom scores (at least three 'moderate' scores) (criteria according to Lindenmayer, Kay \& Opler, 1984). The two subgroups differed significantly in initiation time when figures became more unfamiliar $(\mathrm{F}(2,16)=6.22, \mathrm{p}<0.01)$; this difference approached significance for the factor Complexity $(\mathrm{F}(1,17)=4.37, \mathrm{p}=0.052)$. The two subgroups did not differ significantly in the effects of Familiarity $(\mathrm{F}(1,17)=0.80, \mathrm{p}=0.78)$ and Complexity $(F(1,17)=1.19, p=0.29)$ on reinspection time (see Figure 4B). Figure 4C shows that the effect on pause time resembled the effect on initiation time: the interaction between subgroups of patients and Familiarity was significant $(\mathrm{F}(2,16)=4.90, \mathrm{p}<0.03)$.

\section{Correlations between movement variables and other clinical measures}

Correlations between movement variables and the other clinical rating scores, i.e. positive symptoms, depression and extrapyramidal symptoms, and between movement variables and haldol equivalents of medication were calculated to examine possible associations (see Table 4). The highest correlation between the prescribed dosage of medication and movement variables was for pause time in figure copying $\left(r_{s}=0.35\right)$. Higher scores on positive symptoms were slightly associated with longer initiation times in the figure-copying task. Depression scores were positively related to longer matching times in the DST and longer initiation times in figure copying. Higher scores on the ExtraPyramidal Symptoms scale appeared to be associated with longer initiation times and movement times in line copying. AIMS scores were slightly related to movement distance in the figure-copying task. 
A: initiation time

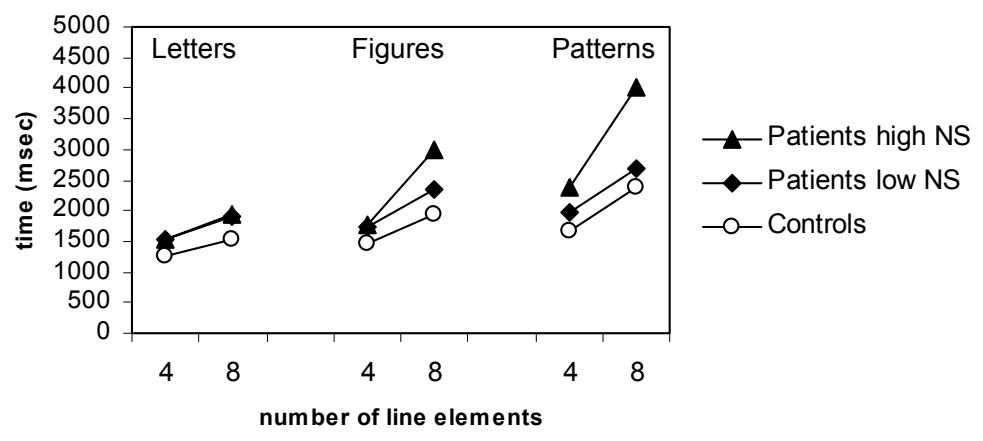

B: reinspection time

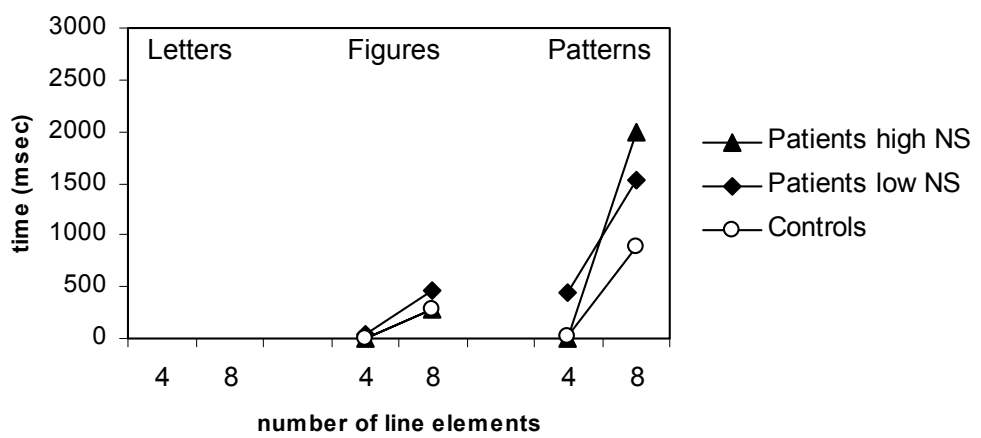

C: pause time

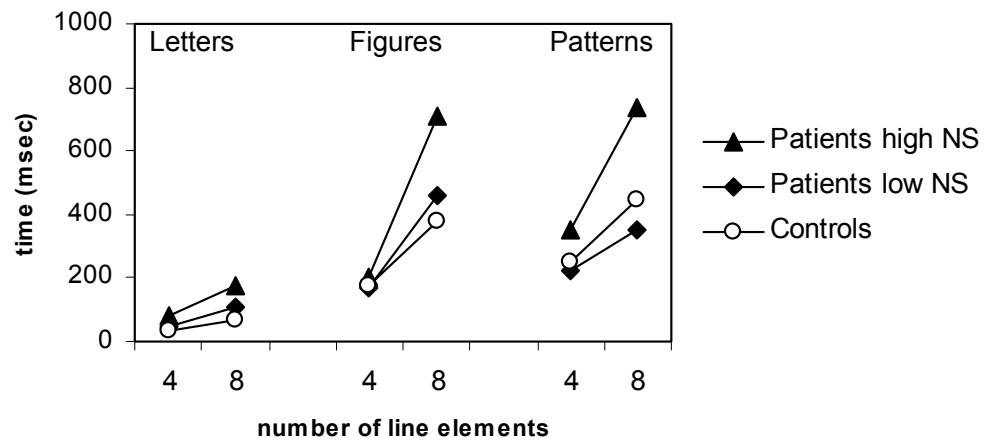

Figure 4: (A) Initiation time with patients divided into two subgroups with either high negative symptom (NS) scores $(n=7)$ or low negative symptom scores $(n=12)$. (B) reinspection time and $(C)$ pause time. 
Table 4: Correlations between clinical rating scores and movement variables. SDS = Self-rating Depression Scale; EPS = ExtraPyramidal Symptoms Scale; AIMS = Abnormal Involuntary Movement Scale.

\begin{tabular}{lclllll}
\hline & Positive & Negative & SDS & EPS & AIMS & Haldol equivalents (mg/day) \\
\hline Lines & & & & & & \\
$\quad$ initiation time & -0.28 & 0.10 & -0.31 & 0.37 & 0.07 & -0.15 \\
$\quad$ movement time & -0.17 & 0.31 & -0.31 & 0.39 & -0.02 & 0.20 \\
movement velocity & -0.05 & $-0.45^{\#}$ & 0.19 & -0.25 & -0.02 & -0.17 \\
$\quad$ distance & -0.08 & -0.13 & 0.05 & 0.22 & -0.25 & 0.12 \\
& & & & & & \\
Figures & & & & & & \\
$\quad$ initiation time & 0.40 & $0.47^{*}$ & 0.35 & 0.08 & -0.21 & 0.19 \\
movement time & 0.16 & 0.21 & 0.11 & 0.28 & -0.18 & 0.13 \\
pen-up time & -0.02 & 0.24 & 0.10 & 0.25 & -0.15 & 0.07 \\
pausing time & 0.19 & 0.39 & 0.22 & 0.11 & 0.22 & 0.35 \\
reinspection time & 0.08 & 0.26 & 0.19 & -0.26 & -0.19 & 0.25 \\
movement velocity & -0.14 & -0.35 & -0.18 & -0.32 & 0.24 & -0.27 \\
distance & 0.05 & -0.23 & -0.12 & -0.05 & -0.38 & -0.19 \\
& & & & & & \\
DST & & & & & & \\
matching time & -0.16 & 0.05 & $-0.41^{\#}$ & -0.15 & 0.08 & 0.08 \\
writing time & -0.01 & 0.26 & -0.26 & 0.21 & -0.04 & 0.02 \\
movement velocity & 0.14 & -0.32 & 0.14 & -0.10 & -0.02 & 0.03 \\
distance & 0.22 & -0.15 & -0.12 & -0.10 & -0.06 & 0.16 \\
\hline * $<0.05, \# p<0.10$ & & & & & &
\end{tabular}

\section{Discussion}

In the present study patients with schizophrenia were clearly slower in both the copying tasks and the Digit Substitution Test (DST) when compared to healthy controls. In the copying tasks, this slowing was not only present in the intervals before starting an action (initiation time) but also during the execution period. In the DST, the patients with schizophrenia were significantly slower in matching the digits. However, no such group difference was found for writing time in this test. When figures to be copied were more unfamiliar and/or complex, patients showed significantly longer reinspection times and movement times. Finally, a correlation was found between negative symptoms and initiation time in the figure-copying task. Closer inspection of this relationship revealed that particularly the most unfamiliar and complex figures proved to cause problems for the patients with clear negative symptoms.

Next the results will be discussed for each of the hypotheses.

Hypothesis 1. Our results confirm the hypothesis of a rather basic, task-independent, psychomotor slowing in patients with schizophrenia. In all three tasks, and thus irrespective of the complexity of the task, patients were approximately one third slower when compared to the controls. This overall slowing is in line with King's theory (1991) which states that psychomotor 
slowing in schizophrenia may in essence be due to a lower speed of basic psychobiological processes. King (1991) and also, more recently, Goldberg and Gold (1995) base this idea on their findings that patients with schizophrenia were even retarded on a simple psychomotor task and did not perform relatively worse on a more complex task when compared to healthy controls. However, it may be questioned whether this general slowing is very specific for schizophrenia, since our data correspond very closely to the results of a meta-analysis made by White et al. (1997) of the performances of patients suffering from depression, which showed that these patients were consistently about $30 \%$ slower than control subjects on a broad range of cognitive tasks. Also our own research on psychomotor slowing in depression (Sabbe et al., 1996b) seems to point to a more or less generalized slowing in depression on different tasks. Thus general psychomotor slowing may not be very specific for schizophrenia, although our more detailed analysis of the DST performances (see discussion of hypothesis 2) revealed clear differences in the type of slowing in schizophrenia and depression (replicating the findings of Van Hoof et al., 1998, see Chapter 2 of this thesis).

Hypothesis 2. The expectation that the patients with schizophrenia would exhibit slowing mainly in those variables that reflect cognitive processing, such as initiation time, reinspection time and duration of pauses during execution in the copying tasks and matching time in the DST, was not unambiguously confirmed.

Initiation time in the copying tasks was significantly longer in the patients with schizophrenia. Furthermore, patient performance on the figure-copying task showed significantly prolonged periods above the paper (pen-up time) and significant slowing in the reinspection time, variables also mainly associated with cognitive processing. No group difference was found for movement velocity, which provides support for the absence of a motor slowing in our patients. Not indisputably in line with a 'cognitive' hypothesis are the significantly longer movement times in the patients with schizophrenia (about 40\%, see Table 3), although, as mentioned before, it would be too straightforward to assume that movement periods solely reflect motor processes. Particularly in the more complex figure-copying task, movement periods may also be used for cognitive processing, such as the preparation and planning of successive movements. However, as movement time was already longer while copying a simple line, we assume that patients with schizophrenia also exhibit a more basic problem with fine motor control. When a person experiences problems with fine motor control he or she may compensate this by increasing the size of his/her writing or drawing, which, however, does not necessarily result in a lower movement velocity. Indeed, our patients tended to write and draw bigger, without being slower than the control group, which is consistent with the results of a recent study on handwriting characteristics in schizophrenia (Gallucci et al., 1997).

On the DST patients performed worse than the controls as appeared from a significantly lower raw score in the first group. Poor performance on the DST in patients with schizophrenia has been reported before (e.g.,Jeste, et al., 1996; Mahurin, Velligan \& Miller, 1998; Brébion et al., 1998). However, the kinematic analysis that we conducted in the present study showed that patients with schizophrenia were slower in matching the digits, but not in writing them down, which may point to an impairment in cognitive processes. In a previous study on the DST, in which we 
compared patients with schizophrenia and patients with a major depression (Van Hoof et al., 1998, see Chapter 2), we found the same pattern of results, viz. cognitive slowing in schizophrenia only. Patients with a major depression showed slowing in both matching and writing.

A noteworthy difference between the findings on the copying tasks and the DST is the relatively strong slowing in matching time (about 44\%, see Table 3) in the patients with schizophrenia when compared to the slowing in initiation time (about 30\%). A likely explanation for this would be that in the copying tasks cognitive processes, such as planning, attention and other processes in memory, are not only involved before, but also during copying, whereas in the DST these cognitive processes are more or less restricted to the matching period. This may also explain the finding that patients with schizophrenia do not show significantly longer movement time in the DST. Movement time in the DST probably expresses more 'pure' and automatic motor performance than it does in the copying tasks.

It can be concluded that, except for the DST, our results are not fully in line with the hypothesis of a more pronounced cognitive slowing in patients with schizophrenia. The findings also suggest impaired fine motor control in schizophrenia.

Hypothesis 3. When stimuli were more difficult, which was the case in our figure-copying task, the patient group did not use significantly more initiation time to memorize and plan the required actions. This appeared to be particularly true for a subgroup of patients with lower scores on negative symptoms; patients with higher scores, on the other hand, did show a marked prolongation of the initiation period (see discussion of hypothesis 4). Further, we also found that the schizophrenia group did reinspect the stimuli longer when these were more unfamiliar, which indicates that they were not able to store the figure in working memory and/or did not plan the required response fully in advance. Together with the fact that the greater part of our patient group, those with lower scores on negative symptoms, did not inspect the stimuli longer before copying (initiation time), seems to suggest a less efficient way of planning (which may also include encoding and storage problems). They start copying relatively fast, irrespective of the complexity of the task. The controls, on the other hand, seem to adjust their initiation time to the task demands in such a way that they are better able to execute the drawing movements in a preplanned sequence. When performing the same figure-copying task, the depression group in our earlier study also did not show prolonged initiation time in copying more complex stimuli (Sabbe et al., 1996b). Comparable to our schizophrenia group, these patients needed more movement time and reinspection time to draw the more complex and unfamiliar figures. This indicates that not only in patients with schizophrenia but also in patients with a depression cognitive processes involved in figure copying seem to be impaired.

When comparing our group findings with the results of a number of studies using tasks that rather differ from ours we find similar results. By measuring changes in force during a reaction time task in which the subjects needed to press a button, Vrtunski et al. (1986) found that in patients with schizophrenia task complexity (ranging from the classification of stimuli to decisions on their referential meaning) appeared to have a larger effect on the execution period than on the initiation period of the reaction. Also studies with a computerized version of the Tower of London showed that a group of patients with schizophrenia was not slower in the initiation period (i.e. planning of 
the first move(s)) of the task (Morris et al., 1995; Hanes et al., 1996; Pantelis et al., 1997), but that they did have a prolonged subsequent execution time (only measured by Pantelis et al., 1997). These authors also mention the possibility that patients with schizophrenia may not plan the required sequence of responses fully in advance, which was supported by their finding that these patients needed more moves to complete the trials and produced fewer perfect solutions. The other explanation given by these authors concerns an insufficiency in holding the information 'on-line' during the execution of the task, which, however, was not supported by significant correlations between the Tower of London task performance and measures of working memory and short-term memory (Morris et al., 1995; Pantelis et al., 1997). It must be noted that in our study the role of an impaired ability to form an internal representation of the stimuli cannot be ruled out, as we did not include a (separate) test for this. This may be seen as a limitation of our study. Therefore, the influence of memory problems on patients' performance, particularly in the more complex figure copying (in line copying subjects were generally not aware of the disappearance of the stimulus), must be addressed in future research.

Hypothesis 4. The final hypothesis of the present study concerned the relationship between negative symptoms and task performance. The severity of negative symptoms appeared to be related to the performance on the figure-copying task, but this held particularly for initiation time. Further examination of this relationship showed that patients with distinct negative symptoms had longer initiation times under more complex task conditions. These patients were particularly challenged when the stimuli became more unfamiliar and, although not significantly, when the stimuli had more line elements. Remarkably, this delayed initiation pattern of our patients with severer negative symptoms bears more striking similarities with the performances of patients with Parkinson's disease on the Tower of London than with those of a schizophrenia group (Pantelis et al., 1997). The patients with Parkinson's disease also needed more time to plan the required movements. However, as negative symptoms were not assessed in the schizophrenia group of Pantelis et al.'s study, no information is available about the relationship between these symptoms and the duration of the first planning period in the Tower of London.

In our study no significant relationship existed between negative symptoms and the DST. This finding accords with the results of Nelson et al. (1990) who did not find a significant difference in the DST performance between groups of either low negative symptom scores or high negative symptom scores. This may indicate that the DST is not sensitive to the effects of negative symptoms of schizophrenia. A comparison between the DST and our copying tasks may illustrate this point: both raw score and matching time appeared to correlate significantly with the time needed to initiate the copying of simple lines, $\rho=-0.44(p<0.02)$ and $\rho=0.39(p<0.03)$, respectively. No significant correlations, however, were found with initiation times in the more complex figure-copying task. Thus, although the DST of course involves various cognitive processes, such as storage of information in working memory and sustained attention, this test does not reflect planning ability in the strict sense of sequence planning. Our figure-copying task, on the other hand, requires the selection and storage of the most efficient sequence of movements varying in difficulty and is therefore more sensitive to the effects of negative symptoms. 


\section{4 | Chapter 4}

Finally, the results of the figure-copying task tentatively suggest that planning problems (including encoding and storage problems) are most striking in patients with higher scores on negative symptoms. If we follow Frith (1992), who views the conversion of a plan into action as one of the main problems in patients with negative symptoms, this would indeed result in longer initiation times. However, our patients with higher negative symptom scores also needed more reinspection time and showed longer pause duration during execution when compared to the controls, which may indicate that, despite their longer initiation time, they were unable to plan all the required movements before copying (a hypothesis also put forward by Morris et al., 1995).

In their reviews King and Green (King \& Green, 1996; Green \& King, 1996) show that antipsychotic medication may have effects on cognitive functioning in schizophrenia. However, only in a minority of studies did they find that antipsychotics negatively affected cognitive function. The majority of studies even showed that antipsychotic medication had positive effects on cognition. Although in our study dosage of antipsychotic medication correlated significantly with negative symptoms (does antipsychotic medication induce negative symptoms or do patients with more severe negative symptoms use higher dosages?), no significant correlation was found between these dosages and the various movement variables. But despite the absence of such a correlation we cannot conclude that antipsychotic medication did not affect our results. Because of their interacting effects with the schizophrenic symptoms, the impact of antipsychotics on cognition in patients with schizophrenia is rather complicated and more ambiguous when compared to the effects of antipsychotics in healthy volunteers (Sharma, 1999). In schizophrenia, antipsychotic medication can directly alter cognitive processing; it may, for instance, bring about an improvement of the disturbed (selective) attention (see Green \& King, 1996). It can also cause sedation, like in healthy subjects, because of the anticholinergic activity of antipsychotics (Green, McElholm \& King, 1996). Sedation, however, was not measured in the present study, so we cannot rule out that the sedative component of the antipsychotic treatment contributed to the psychomotor slowing observed in our patient group. Thirdly, antipsychotic medication often induces extrapyramidal symptoms, some of them resembling the primary negative symptoms of schizophrenia, like flattening of affect and psychomotor poverty. Although our patients did not show clear features of extrapyramidal side effects as measured by means of the EPS and AIMS, an effect on psychomotor slowing cannot be excluded. The design of the present study does not permit us to make clear statements on this issue and future research on the long-term effects of antipsychotic treatment and on the comparison between classical antipsychotics and newer atypical antipsychotics may provide some clarification. Gallhofer et al. (1996), for example, who compared patients treated with atypical antipsychotic medication (clozapine or risperidone) with patients using classical antipsychotic medication (haloperidol or fluphenazine) found that the first group of patients was faster in finding their way through mazes and, most importantly, that they were better able to traverse complex mazes while preserving their motor coordination.

In contrast to the more traditional neuropsychological approach of assessing cognitive and psychomotor functions in schizophrenia, the present study was one of the first in which a more detailed kinematic analysis of these patients' psychomotor performance on drawing tasks was conducted. In recent years there is a growing consensus that standard neuropsychological tests for 
executive functions are too crude and underspecified to catch the specific symptom-related cognitive disturbances in schizophrenia (Frith, 1999). This view opens a challenging new line of research in which a process-oriented approach is used to improve the understanding of the cognitive and motor impairments in patients with schizophrenia (e.g., Morris et al., 1995; Hanes et al., 1996; Gallhofer et al., 1996; Pantelis et al., 1997). We feel that the tasks and kinematic analyses like the ones used in the present study are promising in this line of research.

\section{Acknowledgements}

We wish to thank Stef Grondman for his substantial contribution to the selection of patients and his expertise in doing the PANSS interviews. This study was supported by a grant of the Netherlands Organization for Scientific Research (NWO). 


\section{6 | Chapter 4}

\section{References}

American Psychiatric Association (1987). Diagnostic and statistical manual of mental disorders (3rd edition, revised). Washington, D.C.: APA.

Benson, F. (1990). Psychomotor retardation. Neuropsychiatry, Neuropsychology and Behavioral Neurology, 3, 36-47.

Bermanzohn, P.C., \& Siris, S.G. (1992). Akinesia: A syndrome common to parkinsonism, retarded depression, and negative symptoms of schizophrenia. Comprehensive Psychiatry, 33, 221-232.

Brébion, G., Amador, X., Smith, M.J., \& Gorman, J.M. (1998). Memory impairment and schizophrenia: The role of processing speed. Schizophrenia Research, 30, 31-39.

Crow, T.J. (1980). Molecular pathology of schizophrenia: More than one disease process? British Medical Journal, 280, 66-68.

De Jong, W.P., Hulstijn, W., Jogems-Kosterman, B.J.M., \& Smits-Engelsman B.C.M. (1996). OASIS software and its application in experimental handwriting research. In: M.L. Simner, C.G. Leedman \& A.J.W.M. Thomassen (Eds.), Handwriting and drawing research: Basic and applied issues (pp. 429440). Amsterdam: IOS Press.

Gallucci, R.M., Phillips, J.G., Bradshaw, J.L., Vaddadi, K.S., \& Pantelis, C. (1997). Kinematic analysis of handwriting movements in schizophrenic patients. Biological Psychiatry, 41, 830-833.

Gallhofer, B., Bauer, U., Lis, S., Krieger, S, \& Gruppe, H. (1996). Cognitive dysfunction in schizophrenia: Comparison of treatment with atypical antipsychotic agents and conventional neuroleptic drugs. European Neuropsychopharmacology, 6, S2-13-S2-20.

Goldberg, T.E., \& Gold, J.M. (1995). Neurocognitive deficits in schizophrenia. In: S.R. Hirsch \& D.R. Weinberger (Eds.), Schizophrenia (pp. 146-162). Oxford: Blackwell Science.

Gourovitch, M.L., \& Goldberg, T.E. (1996). Cognitive deficits in schizophrenia: Attention, executive functions, memory and language processing. In: C. Pantelis, H.E. Nelson \& T.R.E. Barnes (Eds.), Schizophrenia: A neuropsychological perspective (pp. 71-86). New York: John Wiley \& Sons.

Green, J.F., \& King, D.J. (1996). Cognitive functioning in schizophrenia: Effects of drug treatments. CNS Drugs 6, 382-398.

Green, J.F., McElholm, A., \& King, D.J. (1996). A comparison of the sedative and amnestic effects of chlorpromazine and lorazepam. Psychopharmacology, 128, 67-73.

Guy, W. (1976) ECDUE Assessment Manual for Psychopharmacology. Rockville: National Institute of Mental Health.

Frith, C.D. (1992). The cognitive neuropsychology of schizophrenia. Hove: Lawrence Erlbaum Associates.

Frith, C.D (1995). Schizophrenia: Functional imaging and cognitive abnormalities. Lancet, 346, 615-620.

Frith, C.D (1999). Commentary on Laws: What are we trying to explain? Cognitive Neuropsychology 4, 3132 .

Hanes, K.R., Andrewes, D.G., Pantelis, C., \& Chiu, E. (1996). Subcortical dysfunction in schizophrenia: A comparison with Parkinson's Disease and Huntington's Disease. Schizophrenia Research, 19, 121-128. 
Hulstijn, W. (1994). Figure copying and retardation in depression. In: C. Faure, P. Keuss, G. Lorette \& A. Vinter (Eds.), Advances in handwriting and drawing: A multidisciplinary approach (pp. 477-488). Paris: Europia.

Hulstijn, W. (1996). Writing and drawing in neuropsychology: Studies on psychomotor slowing. In: M.L. Simner, C.G. Leedham \& A.J.W.M. Thomassen (Eds.), Handwriting and drawing research: Basic and applied issues (pp. 203-214). Amsterdam: IOS Press,.

Jeste, D.V., Heaton, S.C., Paulsen, J.S., Ercoli, L. Harris, M.J., \& Heaton, R.K. (1996). Clinical and neuropsychological comparison of psychotic depression with nonpsychotic depression and schizophrenia. American Journal of Psychiatry, 153, 490-496.

Kay, S.R., Fiszbein, A., \& Opler, L.E. (1987). The Positive and Negative Syndrome Scale (PANSS) for schizophrenia. Schizophrenia Bulletin, 13, 261-275.

King, H.E. (1991). Psychomotor dysfunction in schizophrenia. In: S.R. Steinhauer, J.H. Gruzelier \& J. Zubin (Eds.), Handbook of schizophrenia, Vol. 5: Neuropsychology, psychophysiology and information processing (pp. 273-301). Amsterdam: Elsevier Science Publishers.

King, D.J., \& Green, J.F. (1996). Medication and cognitive functioning in schizophrenia. In: C. Pantelis, H.E. Nelson \& T.R.E. Barnes (Eds.), Schizophrenia: A neuropsychological perspective (pp. 419-444). New York: John Wiley \& Sons.

Lezak, M.D. (1983). Neuropsychological assessment (2nd edition). New York: Oxford University Press.

Lindenmayer, J.P., Kay, S.R. \& Opler, L. (1984). Positive and negative subtypes in acute schizophrenia. Comprehensive Psychiatry, 25, 455-464.

Liddle, P.F. (1987). Schizophrenic syndrome, cognitive performance and neurological dysfunction. Psychological Medicine, 17, 49-57.

Liddle, P.F., \& Morris, D.L. (1991). Schizophrenic syndromes and frontal lobe performance. British Journal of Psychiatry, 158, 340-345.

Mahurin, R.K., Velligan, D.I., \& Miller, A.L. (1998). Executive-frontal lobe cognitive dysfunction in schizophrenia: A symptom subtype analysis. Psychiatry Research, 79, 139-149.

Malla, A.K., Norman, R.M.G., Aguilar, O., Carnahan, H., \& Cortese, L. (1995). Relationship between movement planning and psychopathology profiles in schizophrenia. British Journal of Psychiatry, 167, 211-215.

Morris, R.G., Rushe, T., Woodruffe, P.W.R., \& Murray, R.M. (1995). Problem solving in schizophrenia: A specific deficit in planning ability. Schizophrenia Research, 14, 235-246.

Nelson, H.E. Pantelis, C., Carruthers, K., Spellers, J., Baxendale, S., \& Barnes, T.R.E. (1990). Cognitive functioning and symptomatology in chronic schizophrenia. Psychological Medicine, 20, 357-365.

Pantelis, C., Barnes, T.R.E., Nelson, H.E., Tanner, S., Weatherley, L., Owen, A.M., \& Robbins, T.W. (1997). Frontal-striatal cognitive deficits in patients with chronic schizophrenia. Brain 120, 1823-1843.

Sabbe, B.G.C., Van Hoof, J.J.M., Hulstijn, W., \& Zitman, F.G. (1996a). Changes in fine motor retardation in depressed patients treated with fluoxetine. Journal of Affective Disorders, 40, 149-158.

Sabbe, B.G.C., Hulstijn, W., Van Hoof, J.J.M., \& Zitman, F.G. (1996b). Fine motor retardation and depression. Journal of Psychiatric Research, 30, 295-306. 
Sabbe, B.G.C., Van Hoof, J.J.M., Hulstijn, W., \& Zitman, F.G. (1997). Depressive retardation and treatment with fluoxetine: Assessment of the motor component. Journal of Affective Disorders, 43, 53-62.

Sabbe, B.G.C., Hulstijn, W., Van Hoof, J.J.M., Tuynman-Qua, H.G., \& Zitman, F.G. (1999). Retardation in depression: Assessment by means of simple motor tasks. Journal of Affective Disorders, 55, 39-44.

Shallice, T. (1982). Specific impairments of planning. Philosophical transactions of the Royal Society of London series B Biological sciences, 298, 199-209.

Shapiro, M.B., \& Nelson, E.H. (1955). An investigation of the nature of cognitive impairment in cooperative psychiatric patients. British Journal of Psychiatry, 28, 239-256.

Sharma, T. (1999). Cognitive effects of conventional and atypical antipsychotics in schizophrenia. British Journal of Psychiatry, 174 (suppl. 38), 44-51.

Simpson G.M., \& Angus J.W.S. (1970). A rating scale for extrapyramidal side effects. Acta Psychiatrica Scandinavica, 212 (suppl), 11-19.

Smith, J.M., Kucharski, L.T., Oswald, W.T., \& Waterman, L.J. (1979). A systematic investigation of tardive dyskinesia in inpatients. American Journal of Psychiatry, 136, 918-922.

Stroop, J.R. (1935). Studies of interference in serial verbal reactions. Journal of Experimental Psychology, $18,643-662$.

Teulings, H., \& Maarse, F.J. (1984). Digital recording and processing of handwriting movements. Human Movement Science, 3, 193-217.

Van Galen, G.P. (1991). Handwriting: Issues for a psychomotor theory. Human Movement Science, 10, 165191.

Van Hoof, J.J.M., Hulstijn, W., Van Mier, H., \& Pagen, M. (1993). Figure drawing and psychomotor retardation: Preliminary report. Journal of Affective Disorders, 29, 263-266.

Van Hoof, J.J.M., Jogems-Kosterman, B.J.M., Sabbe, B.G.C., Zitman, F.G., \& Hulstijn, W. (1998). Differentiation of cognitive and motor slowing in the Digit Symbol Test (DST): Differences between depression and schizophrenia. Journal of Psychiatric Research, 32, 99-103.

Van Mier, H., \& Hulstijn, W. (1993). The effects of motor complexity and practice on initiation time in writing and drawing. Acta Psychologica, 84, 231-251.

Van Sommers, P. (1989). A system for drawing and drawing-related neuropsychology. Cognitive Neuropsychologica, 6, 117-164.

Vrtunski, P.B., Simpson, D.M., Weiss, K.M., \& Davis, G.C. (1986). Abnormalities of fine motor control in schizophrenia. Psychiatry Research, 18, 275-284.

Wechsler, D. (1956). Manual for the Wechsler Adult Intelligence Scale. New York: Psychological corporation.

White, D.A., Myerson, J., \& Hale, S. (1997). How cognitive is psychomotor slowing in depression? Evidence from a meta-analysis. Aging, Neuropsychology, and Cognition, 4, 166-174.

Widlöcher, D., \& Hardy-Bayle, M.C. (1989). Cognition and control of action in psychopathology. European Bulletin of Cognitive Psychology, 9, 583-615.

Wolkin, A., Sanfilipo, M., Wolf, A.P., Angrist, B., Brodie, J.D., \& Rotrosen, J. (1992). Negative symptoms and hypofrontality in chronic schizophrenia. Archives of General Psychiatry, 49, 959-965. 
Psychomotor slowing and planning deficits in schizophrenia $\mid 79$

Zung, W.W.K. (1965) A Self-rating Depression Scale. Archives of General Psychiatry, 12, 63-70. 


\title{
Chapter 5
}

\section{Is the planning of action more impaired in schizophrenic patients than in depressed patients? The effects of conflicting graphic production rules ${ }^{1}$}

\begin{abstract}
The present study examined the question whether the (im)possibility of applying graphic production rules would influence the speed of copying simple patterns in schizophrenic and depressed patients. It was hypothesized that because of a lack of planning schizophrenic patients would show fewer differences between copying 'nonconflicting' patterns (in which rules can be applied) and 'conflicting' patterns (in which rules have to be violated) when compared to depressed patients and controls. The results generally confirmed these predictions, in that differences between non-conflicting and conflicting patterns were only found in depressed patients and controls. This opens a new way for the study of planning deficits in schizophrenia.
\end{abstract}

\footnotetext{
${ }^{1}$ Based on:
}

Jogems-Kosterman, B.J.M., Hulstijn, W., Van Hoof, J.J.M., \& Thomassen, A.J.W.M. (1999). In: G. Leedham, M. Leung, V. Sagar \& X. Xuhong (Eds.), Proceedings of the 9th Biennial Conference of the International Graphonomics Society (pp. 225-229). Singapore: NTU. 


\section{Introduction}

Both schizophrenia and depression are characterized by a general slowing of cognitive and motor activities. This 'psychomotor slowing' has been viewed by some authors as the manifestation of a very general syndrome underlying several psychiatric and neurological diseases (Benson, 1990; Bermanzohn \& Siris, 1992). Others, however, emphasize the qualitative differences in psychomotor slowing between schizophrenia and depression. An interesting difference, proposed by Widlöcher and Hardy-Bayle (1989), is to regard psychomotor slowing in depression as the result of a lack of activation, and in schizophrenia as the result of an inability to plan and organize actions. This theorizing is more or less in line with the one proposed by Frith (1992), who regards the main problems in schizophrenia as arising from failures in the self-generation of action (and less in the ability to react to external stimuli), while psychomotor slowing in depression is viewed as resulting from less activity in a system that mediates self motivated behavior. Consequently, the psychomotor slowing in depression manifests itself not just in planning activities but in almost all behavioral aspects.

Our own research into psychomotor slowing in depression (Van Hoof et al., 1993; Sabbe et al., 1996a, 1996b, 1997, 1999) has indeed confirmed that depressed patients are retarded in simple motor tasks as well as in tasks involving more complex cognitive operations like the copying of unfamiliar figures. In our latest studies on the nature of psychomotor slowing in schizophrenia (Van Hoof et al., 1998, see Chapter 2; Jogems-Kosterman et al., 2001, see Chapter 4), it was found that schizophrenic patients are only delayed on those aspects of the task that reflect cognitive processes. Remarkably, schizophrenic patients did not show relatively longer initiation times when copying more complex patterns, but they had to reinspect the patterns more often during drawing. This may be seen as a less efficient way of planning.

Planning of action may take place at a high cognitive level, but may also be governed by more physiological and physical factors. In the domain of writing and drawing research, there is convincing evidence that our graphic behavior is influenced by certain graphic production rules (Goodnow \& Levine, 1973; Thomassen \& Tibosch, 1991). The existence of these rules shows that subjects do not plan motor actions ad hoc, but rather select an appropriate sequence of movements. Some of these rules may reflect a higher cognitive strategy, such as anchoring a line onto a previous line which requires advance-planning, whereas others may be more concerned with movement efficiency such as threading lines without pen-lifts. Also cultural and educational factors, such as the direction of reading and writing of course have their influence on these rules. Even in drawing simple patterns, consisting of only two or three lines, these graphic production rules are obeyed (Thomassen \& Tibosch, 1991). It has been found that the possibility (or impossibility) of applying these rules effects the speed of copying (Thomassen, Meulenbroek \& Tibosch, 1991). Copying patterns in which the rules cannot be fully applied ('conflicting' patterns) leads to longer hesitations above the paper during copying, when compared to patterns that can be copied according to the rules ('non-conflicting' patterns).

In the present study we used these differences in hesitations above the paper between conflicting and non-conflicting patterns as a means to investigate the amount of rule governed behavior, as one aspect of planning behavior. Because of the disturbed planning and organization of 
action in schizophrenic patients that is frequently observed in clinical practice, we predicted that the copying behavior of these patients will be less guided by the graphic production rules found in healthy persons. They will therefore show less difference in copying time between 'non-conflicting' and 'conflicting' patterns. Depressed patients, on the other hand, will not be different from healthy persons with respect to the influence of conflicting rules. They will just be slowed in general, both in the initiation and in the duration of copying.

\section{Method}

\section{Subjects}

In this study, the performances were compared of 30 patients with schizophrenia ${ }^{2}$ and 30 patients with a major depressive episode ${ }^{3}$ (diagnosed according to DSM-III-R, American Psychiatric Association, 1987). All patients were under normal psychopharmacological treatment. Each patient group was compared with a separate control group of healthy volunteers. See Table 2 for more detailed information.

Table 2: Number of subjects, distribution of sex, mean age and median of educational level in the study groups.

\begin{tabular}{lllll}
\hline Group & Number & Sex & Age in years (SD) & Education \\
\hline Schizophrenia & 30 & $27 ; 3$ & $33.8(9.2)$ & 5 \\
Controls & 20 & $10 ; 10$ & $40.7(11.0)$ & 6 \\
Depression & 30 & $8 ; 22$ & $40.6(11.1)$ & 4 \\
Controls & 22 & $8 ; 14$ & $41.6(10.8)$ & 5 \\
\hline
\end{tabular}

\section{Task and apparatus}

The subjects performed a drawing task consisting of copying simple patterns of two or three line segments. The stimuli were 12 patterns and their matched mirror images, in which the first could be copied according to the graphic production rules, whereas copying the mirror image would lead to conflicts between these rules. Figure 1 shows an example of such a pair. On the left is displayed a 'non-conflicting' (NC) pattern and on the right a 'conflicting' (C) pattern.

The 24 patterns were presented, in random order, on a computer screen that was placed in front of the subject. They had to be copied on normal A-4 paper, within $3 \times 4 \mathrm{~cm}$ boxes. The pen movements were registered by means of a personal computer, a WACOM 1218RE digitizer and a special pressure-sensitive pen. The subjects were instructed to hold the pen in a small circle at the lower left corner of the box (see also Figure 1). While holding the pen in this position for a

\footnotetext{
${ }^{2}$ These patients participated in an extensive study to predict the outcome of dehospitalisation by means of a neuropsychological assessment (Fakkers et al., 2002).

3 These patients were included in a study in which the effects of antidepressant medication on psychomotor slowing were investigated.
} 
(random) period between 500 to $2000 \mathrm{~ms}$, the stimulus appeared on the screen guided by a warning signal $(1000 \mathrm{~Hz}, 300 \mathrm{~ms})$. The subjects had to copy the stimulus within the box at a fast speed. As soon as the subject started drawing the stimulus disappeared from the screen. After completing the drawing, the pen had to be placed in a circle at the upper right corner of the box for at least $300 \mathrm{~ms}$.
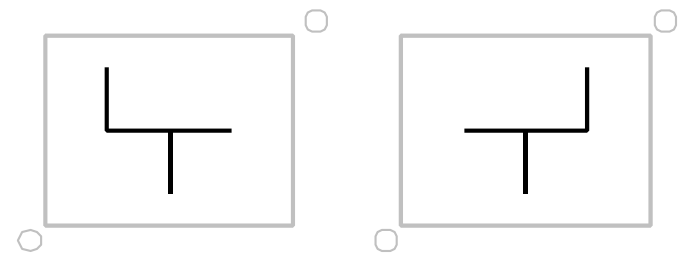

Figure 1: Examples of a 'non-conflicting' pattern (left) and a 'conflicting' pattern (right) used in this study.

\section{Recording and analyses}

The $\mathrm{x}$ and $\mathrm{y}$ coordinates of the pen tip were sampled with a frequency of $200 \mathrm{~Hz}$ and a spatial accuracy of $0.2 \mathrm{~mm}$. In line with the study of Thomassen et al. (1991), the following dependent variables were calculated for each copied pattern:

Initiation time (IT): the duration of the period between the appearance of the stimulus on the computer screen and the first pen-down period.

Movement time (MT): the duration of the periods in which the pen was moving or pausing on the paper during the production of the pattern.

Pen-up time (UpT): the duration of the periods in which the pen was above the paper during the production of the pattern.

Within each study group, paired-samples $t$-tests were used to analyze the differences between the $\mathrm{NC}$ and $\mathrm{C}$ patterns for the obtained variables. We also tested the differences between each patient group and their control group and between schizophrenia and depression using ANOVA's with group as between-subject factor and condition $(\mathrm{NC}, \mathrm{C})$ as within-subject factor.

\section{Results}

The mean values of IT, MT and UpT for the NC and C patterns for the four groups are visualized in Figure 2. Only the depressed patients show a significant difference in IT between the NC and C patterns. Significantly longer UpT's were obtained for the conflicting patterns in all groups except from the schizophrenic patients. No significant differences appeared for MT.

The results of the ANOVA's for IT, MT and UpT are displayed in Table 3. The schizophrenic patients showed significantly longer IT's, MT's and UpT's when compared to their control group. As predicted, the difference in UpT between NC and C patterns was larger in control subjects but the interaction between group and condition was not significant. However, when controlling for the overall larger production time (MT plus UpT) of the schizophrenia group, by expressing the UpT as 
Is the planning of action more impaired in schizophrenic patients than in depressed patients? $\mid 85$ a percentage of the total production time, produced a group by conflict interaction that approached significance $(\mathrm{p}=0.075)$.
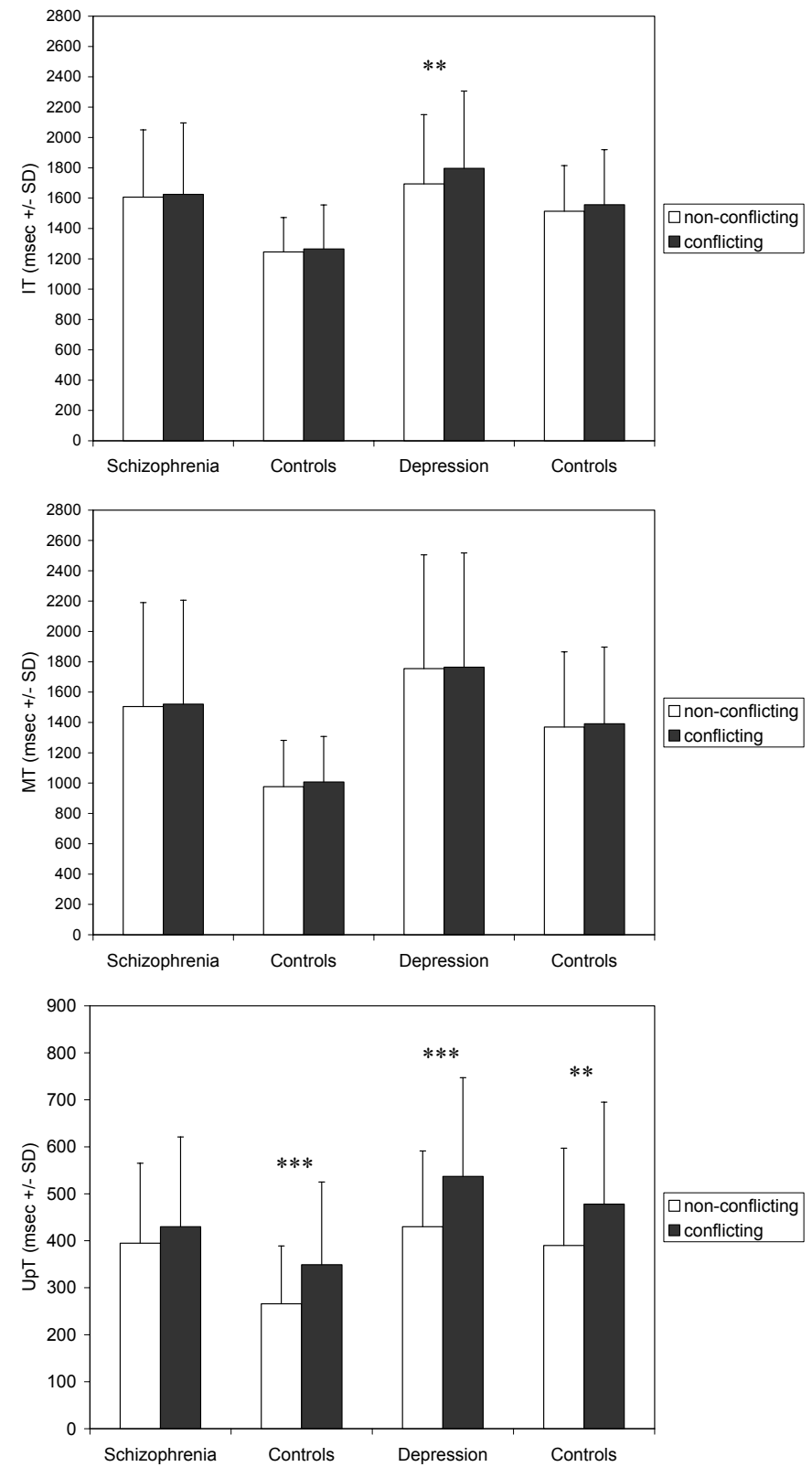

Figure 2: The mean duration of initiation time (IT), movement time (MT), and Pen-up time (UpT) for the $\mathrm{NC}$ and $\mathrm{C}$ patterns for the different groups. ${ }^{* *} \mathrm{p}<0.001, * * \mathrm{p}<0.01,{ }^{*} \mathrm{p}<0.05$ 
The depressed patients were significantly slower in IT and MT, but not in UpT, than their controls. The interesting difference between $\mathrm{C}$ and $\mathrm{NC}$ patterns in UpT for depressed patients was as large as the difference of their control subjects. When comparing the two patient groups, no significant difference was found for any of the three variables, although the difference in UpT approached significance $(\mathrm{p}=0.076)$. The group by condition interaction was significant for IT and approached significance for UpT $(\mathrm{p}=0.07)$ and for UpT expressed as a percentage of total production time $(\mathrm{p}=0.053)$. Apparently, the difference between copying $\mathrm{C}$ and $\mathrm{NC}$ patterns was larger for IT and for UpT in depressive patients than in schizophrenic patients.

Table 3: Results of the analyses of variance for IT, MT and UpT.

\begin{tabular}{lccc}
\hline & \multicolumn{3}{c}{ Variable } \\
\cline { 2 - 4 } & IT & MT & UpT \\
\hline Group (Schizophrenia, Controls) & $10.38^{* *}$ & $10.29 * *$ & $5.62 *$ \\
Condition (C, NC) & 1.31 & 1.66 & $7.88^{* *}$ \\
Group X Condition & 1.00 & 1.70 & 1.30 \\
& & & \\
Group (Depression, Controls) & $4.63^{*}$ & $5.32 *$ & 1.19 \\
Condition (C, NC) & $8.21^{* *}$ & 3.50 & $24.26^{* * *}$ \\
Group X Condition & 1.26 & 0.90 & $0.36^{*}$ \\
& & & $3.26^{*}$ \\
Group (Schizophrenia, Depression) & 2.14 & 2.61 & $12.69^{* * *}$ \\
Condition (C, NC) & $10.94 * *$ & 2.30 & $3.42^{\#}$ \\
Group X Condition & $5.00^{*}$ & 0.30 & \\
$* * * \mathrm{p}<0.001, * * \mathrm{p}<0.01, * \mathrm{p}<0.05, \# \mathrm{p}<0.10$ & &
\end{tabular}

\section{Discussion}

In this study, the copying of 'conflicting' patterns led to a significantly longer initiation time (IT) in the depressed patients and in an even more pronounced longer Pen-up time (UpT) in both depressed patients and control groups. Our prediction that depressed patients would show the same effects of conflicting rules as healthy subjects was confirmed. Also in line with our prediction, no significant difference in UpT between the NC and C patterns was found for the schizophrenia group. However, this difference between $\mathrm{NC}$ and $\mathrm{C}$ was not significantly smaller than that in their control group, although when controlling for overall production time, this difference approached significance. In addition, when compared to the depressed patients, the schizophrenic patients showed a significantly smaller difference in initiation time between 'conflicting' and 'non-conflicting' patterns, and, although only approaching significance, the same tendency could be seen for UpT.

With respect to the overall group differences, averaged over both types of patterns, it was found that the schizophrenic patients were slower in IT, MT and UpT when compared to their control group. Also the depressed group showed longer IT's and MT's than their control group, which is in line with earlier findings with copying tasks (Van Hoof et al., 1993; Sabbe et al., 1996a, 
1996b, 1997, 1999). No significant overall group differences were found between schizophrenia and depression. It has to be noted that the two control groups differed significantly with respect to both initiation time and duration of copying. This was unexpected and we suspect that this difference might be due to a different interpretation of the instruction by these subjects, in the sense that the control group of the depressed patients might have valued accuracy more than a fast speed of copying. Another factor may be that the controls of the schizophrenic patients were staff members of our own institute, who therefore were more familiar with the test method, whereas the control group of the depressed patients consisted for the greater part of employees of a pharmaceutical company. However, combining the two control groups into one group of 42 subjects resulted in similar interaction effects between group (schizophrenia vs. controls) and condition for the various movement variables.

The important finding was that the groups tended to differ in how they produced the 'conflicting' versus the 'non-conflicting' patterns. Schizophrenic patients seem to have fewer problems with conflicting rules, which may confirm the idea that they do not plan their actions fully (Widlöcher \& Hardy-Bayle, 1989; Frith, 1992). It is possible that they select the order and direction of the strokes more or less randomly. An additional analysis of the sequencing of their drawing must illuminate to what extent schizophrenic patients follow graphic production rules. Are they using all rules to a lesser degree or are there specific rules, such as those reflecting more cognitive strategies, which are not followed? Or do they even omit rules reflecting more automatic processes? There is evidence from autobiographic reports (McGhie \& Chapman, 1961) and neuropsychological studies (John \& Hemsley, 1992) that schizophrenic patients suffer from a loss of automatisms.

Therefore this study prompts additional analyses of sequencing and planning in graphic production. As such it may be seen as a first step on a new methodological path. So far, research on graphic production has not been used in the study of deficient planning in schizophrenia, and compared with tests like the Tower of London, the study of the grammar of action in graphic production has two major advantages. First, graphic production rules range over more than one level, from low level movement constraints to higher cognitive principles. Second graphic production can be recorded quite easily allowing detailed kinematic analyzing.

\section{Acknowledgements}

The present study was supported in part by a grant of Organon International BV. 


\section{8 | Chapter 5}

\section{References}

American Psychiatric Association (1987). Diagnostic and statistical manual of mental disorders (3rd edition, revised). Washington, D.C.: APA.

Benson, F. (1990). Psychomotor retardation. Neuropsychiatry, Neuropsychology, and Behavioral Neurology, 3, 36-47.

Bermanzohn, P.C., \& Siris, S.G. (1992). Akinesia: A syndrome common to parkinsonism, retarded depression, and negative symptoms of schizophrenia. Comprehensive Psychiatry, 33, 221-232.

Fakkers, G.P.F.M., Jogems-Kosterman, B.J.M., Loonen, A.J.M., Van Hoof, J.J.M., \& Hulstijn, W. (2002). Changes in clinical functioning and neurocognitive performances in patients with schizophrenia during the first years of deinstitutionalization. Schizophrenia Research, 53, 127.

Frith, C.D. (1992). The cognitive neuropsychology of schizophrenia. Hove: Lawrence Erlbaum Associates.

Goodnow, J.J., \& Levine, R.A. (1973). The grammar of action: Sequence and syntax in children's copying. Cognitive Psychology, 4, 82-98.

Jogems-Kosterman, B.J.M., Zitman, F.G., Van Hoof, J.J.M., \& Hulstijn, W. (2001). Psychomotor slowing and planning deficits in schizophrenia. Schizophrenia Research, 48, 317-333.

John, C.H., \& Hemsley, D.R. (1992). Gestalt perception in schizophrenia. European Archives of Psychiatry and Clinical Neuroscience, 241, 215-221.

McGhie, A., \& Chapman, J. (1961). Disorders of attention and perception in early schizophrenia. British Journal of Medical Psychology, 34, 103-116.

Sabbe, B.G.C., Van Hoof, J.J.M., Hulstijn, W., \& Zitman, F.G. (1996a). Changes in fine motor retardation in depressed patients treated with fluoxetine. Journal of Affective Disorders, 40, 149-158.

Sabbe, B.G.C., Hulstijn, W., Van Hoof, J.J.M., \& Zitman, F.G. (1996b). Fine motor retardation and depression. Journal of Psychiatric Research, 30, 295-306.

Sabbe, B.G.C., Van Hoof, J.J.M., Hulstijn, W., \& Zitman, F.G. (1997). Depressive retardation and treatment with fluoxetine: Assessment of the motor component. Journal of Affective Disorders, 43, 53-62.

Sabbe, B.G.C., Hulstijn, W., Van Hoof, J.J.M., Tuynman-Qua, H.G., \& Zitman, F.G. (1999). Retardation in depression: Assessment by means of simple motor tasks. Journal of Affective Disorders, 55, 39-44.

Thomassen, A.J.W.M., \& Tibosch, H.J.C.M. (1991). A quantitative model of graphic production. In: J. Requin \& G.E. Stelmach (eds.), Tutorials in motor neuroscience (pp. 269-282). Dordrecht: Kluwer Academic Publishers.

Thomassen, A.J.W.M., Meulenbroek, R.G.J., \& Tibosch, H.J.C.M. (1991). Latencies and kinematics reflect graphic production rules. Human Movement Science, 10, 271-289.

Van Hoof, J.J.M., Hulstijn, W., Van Mier, H., \& Pagen, M. (1993). Figure drawing and psychomotor retardation: Preliminary report. Journal of Affective Disorders, 29, 263-266.

Van Hoof, J.J.M., Jogems-Kosterman, B.J.M., Sabbe, B.G.C., Zitman, F.G., \& Hulstijn, W. (1998). Differentiation of cognitive and motor slowing in the Digit Symbol Test (DST): Differences between depression and schizophrenia. Journal of Psychiatric Research, 32, 99-103.

Widlöcher, D., \& Hardy-Bayle, M.C. (1989). Cognition and control of action in psychopathology. European Bulletin of Cognitive Psychology, 9, 583-615. 


\title{
Chapter 6
}

\section{Planning in graphic production in patients with schizophrenia: Conflicting results?}

\begin{abstract}
Although schizophrenia is clearly associated with impaired planning ability, the individual manifestations of the impairment may vary considerably. Indeed, our previous report on planning ability in figure copying suggested differences between subgroups of patients with schizophrenia (Hulstijn et al., 2001). The present study was designed to illuminate these differences. The figure-copying task applied consisted of patterns that varied in the presence or absence of conflict they provoked with normal preferences, the so-called graphic production rules. It was assumed that for the copying of 'non-conflicting' patterns, in which the main graphic production rules can be followed, a routine and automatic strategy suffices, whereas copying 'conflicting' patterns, in which these rules conflict with the task demands, might reveal planning failure and problems with the anticipation of these conflicts. It was found that the performance of a group of schizophrenia inpatients paralleled that of a group of depressed patients more than the performance of a group of patients with schizophrenia scheduled to be released from the psychiatric institute (future outpatients). Whereas both the inpatients and the depressed patients particularly showed prolonged hesitations above the paper during the copying of conflicting patterns, the future outpatients did not. The results of the present study indicated that this distinction between subgroups of patients with schizophrenia could not be explained by differences in psychopathology or depressive symptoms in particular, but rather by a psychomotor slowing that may underlie these symptoms. Our findings suggest that patients with schizophrenia that do not exhibit characteristics of psychomotor slowing tend to use a more automatic or stereotyped reaction pattern, while patients that do, take longer to decide which sequencing pattern is most convenient.
\end{abstract}




\section{Introduction}

Schizophrenia is characterized by a general slowing of both cognitive and motor actions. This slowing also referred to as the 'psychomotor poverty' syndrome (Liddle, 1987) is mostly viewed as one of the hallmarks of the illness and considered to be a main feature of the negative symptoms of schizophrenia (Crow, 1980). Manifestations of psychomotor slowing are not restricted to schizophrenia and can also be observed in other psychiatric and neurological diseases (Benson, 1990; Bermanzohn \& Siris, 1992). For example, patients with a major depression demonstrate psychomotor retardation in a wide range of activities, such as in gesturing, walking and speech. However, according to some authors (Widlöcher \& Hardy-Bayle, 1989; Frith, 1992), the causes of psychomotor slowing in schizophrenia and depression are not the same. Whereas patients with schizophrenia are thought to primarily have problems with the planning and organization of selfgenerated behavior, depressed patients more or less lack the energy to fulfill self-motivated activities. Understanding the planning difficulties in schizophrenia is complicated by the fact that it may be expressed in very diverse ways. Some patients lack the goals to perform self-directed actions. Others do have set goals but they are unable to inhibit their reactions to inappropriate stimuli, which may lead to either stereotyped or disorganized behavior (Frith, 1992).

Although the theories of Widlöcher and Hardy-Bayle (1989) and Frith (1992) point to an important difference between schizophrenia and depression in relation to self-directed behavior, impaired planning ability has been shown to feature in both schizophrenia (Morris et al., 1995; Hanes et al., 1996; Pantelis et al., 1997; Gallhofer et al., 1996) and depression (Elliott, 1997). One of the difficulties with finding experimental data supporting differences between patient groups is that most planning tasks are complex and draw on several cognitive processes, which complicates the interpretation of the test performance. Explicit planning is needed in tasks in which a more or less routine response is insufficient and in which the possible solutions are not readily apparent or seem to be equally appropriate. To come up with the best solution, a person has to use a selfdirected strategy of deliberation, weighing all possible solutions, and needs to be able to put the various elements of the most suitable solution in the appropriate order. Problems may occur in one or more of the subprocesses, such as in working memory, sequencing, attention and/or monitoring.

Most planning tests, such as the Tower of London (originally designed by Shallice, 1982; also see Morris et al., 1995; Hanes et al., 1996; Pantelis et al., 1997; Marczewski, Van der Linden \& Larøi, 2001), maze tests (Gallhofer et al., 1996) and our own complex figure-copying task (JogemsKosterman et al., 2001, see Chapter 4 of this thesis) are quite complex tasks that rely upon several cognitive processes, which makes it difficult to quantify likely differences in planning behavior between patient groups. In an attempt to gain more insight into these assumed differences, we recently applied a relatively simple copying task to compare planning ability in schizophrenia and depression (Jogems-Kosterman et al., 1999, see Chapter 5 of this thesis). The choice of a copying task is based on the idea that our graphic behavior is governed by so-called graphic production rules (Thomassen \& Tibosch, 1991; Thomassen, Meulenbroek \& Tibosch, 1991). These rules incorporate rather implicit preferences with respect to starting points, stroke directions and stroke sequences. In copying certain patterns, like the letter L, these graphic production rules can more or less be easily applied. However, when copying other patterns, for example the same 'easy' patterns that are now 
mirrored around the Y-axis, conflicts between rules may arise. It has been demonstrated that such conflicts influence the copying speed (Thomassen, Meulenbroek \& Tibosch, 1991). Copying 'conflicting' patterns results in somewhat longer latencies before the start of the drawing and in significantly longer hesitations above the paper during execution.

Problems with a flexible application of these graphic production rules may be expressed in several ways. First, the person involved may not even consider that there are a number of alternatives and will consequently stick to the most dominant rule. This will lead to a relatively small effect on the initiation time and the duration of subsequent hesitations above the paper during drawing (the so-called pen-up time). Secondly, the individual does recognize the alternatives, yet is not able to decide quickly. This will result in a prolongation of both initiation time and pen-up time. A third possibility is that, initially, the person does not consider the alternatives but discovers these during the execution. In this case, no effect may be seen on initiation time, whereas the pen-up time might be substantially prolonged.

Table 1: Differences in the mean values of initiation time and pen-up time between conflicting (C) and nonconflicting patterns (NC) for each of the groups studied (Based on Figures 3 and 4 in Hulstijn et al., 2001).

\begin{tabular}{|c|c|c|c|c|}
\hline Patiënt groups & Initiation tir & & Pen-up time & \\
\hline & Difference & P-level & Difference & P-level \\
\hline Controls $^{1}$ & $\mathrm{C}=\mathrm{NC}$ & & $\mathrm{C}>\mathrm{NC}$ & $* * *$ \\
\hline Depression $^{1}$ & $\mathrm{C}>\mathrm{NC}$ & $* *$ & $\mathrm{C}>\mathrm{NC}$ & $* * *$ \\
\hline Schizophrenia-Out ${ }^{1}$ & $\mathrm{C}=\mathrm{NC}$ & & $\mathrm{C}=\mathrm{NC}$ & \\
\hline Schizophrenia-In I & $\mathrm{C}=\mathrm{NC}$ & & $\mathrm{C}>\mathrm{NC}$ & $* * *$ \\
\hline Schizophrenia-In II & $\mathrm{C}>\mathrm{NC}$ & $* *$ & $\mathrm{C}>\mathrm{NC}$ & $* *$ \\
\hline Schizophrenia-In III & $\mathrm{C}>\mathrm{NC}$ & * & $\mathrm{C}>\mathrm{NC}$ & ** \\
\hline
\end{tabular}

In an earlier study (Jogems-Kosterman et al., 1999, see Chapter 5) aimed at investigating planning ability in schizophrenia, we found that the effects of conflicting rules tended to be smaller in a schizophrenia group when compared to a group of patients with a major depression and a group of healthy controls. The depressed patients behaved more similarly to the control subjects and only demonstrated a general slowing. We suggested that the group of patients with schizophrenia, which consisted of patients selected by their psychiatrist to leave the hospital, experienced fewer problems with conflicting rules because they did not fully plan their actions (cf. the theory of Widlöcher \& Hardy-Bayle, 1989; Frith, 1992). They, in fact, may not perceive that the task demands are in conflict with the graphic rules: with respect to choosing a starting point they simply follow the most dominant rule. Later observations in other groups of patients with schizophrenia (Hulstijn et al., 2001), however, did not confirm these earlier findings. Remarkably, the performances of these latter groups, all inpatients, more strongly resembled those of the depressed patients (see Table 1). In contrast to the outpatients studied by Jogems-Kosterman et al. (1999), most inpatient groups (II and III) showed significant differences in initiation time between non-conflicting and conflicting 


\section{2 | Chapter 6}

patterns. Further, all three inpatient groups needed significantly more pen-up time to copy the conflicting patterns.

The purpose of the present study was to try and clarify these differences between patient groups. To this end, we directly compared the performances of two subgroups of patients with schizophrenia, i.e. the subgroup of patients selected by their psychiatrist to move to a residence outside the psychiatric institute involved in the study by Jogems-Kosterman et al. (1999; see Table 1), and a subgroup of patients that was to remain in the institute (group II; see Table 1). These two patient groups participated in an extensive study aimed at examining whether cognitive functions were predictive of functional outcome after deinstitutionalization (Fakkers et al., 2002). We further contrasted the performances of these patients with the initial group of depressed patients (JogemsKosterman et al., 1999).

The first question we tried to find an answer to was whether a differentiation between the two subgroups of patients with schizophrenia could be explained by differences in clinical ratings. As the performances of the groups of inpatients corresponded more closely to those of the depression group, it was assumed that these inpatients might display more severe psychopathology and particularly marked depressive symptoms.

The second question we investigated was whether we could find proof showing that a substantial part of the patients with schizophrenia displayed a similar slowing as that underlying depression. According to Brébion et al. (2000), psychomotor slowing is an important feature of schizophrenia but might have the characteristics of a 'depressive slowing'. To examine psychomotor speed, we therefore included the analyses of two 'typical' psychomotor tasks for the patient groups: the Digit Symbol Test (Wechsler, 1956), which is a standard test assessing psychomotor speed, and a goal-directed line-drawing task.

Third, in contrast to our first study (Jogems-Kosterman et al., 1999), we now carefully selected from the original set of 12 'non-conflicting' (NC) and 'conflicting' (C) pairs, those threesegment patterns that were most sensitive to the effects of conflicting rules.

\section{Method}

\section{Participants}

In this study we compared the performances of patients diagnosed with schizophrenia with those of patients with a major depression (diagnoses according to DSM IIII-R, American Psychiatric Association, 1987). The performance of a group of healthy volunteers was used for control purposes. The group of patients with schizophrenia consisted of two subgroups, i.e. one subgroup of patients that were to remain hospitalized and another subgroup of patients selected by their psychiatrist to move to a residence outside the institute (Fakkers et al., 2002). The eligibility criteria for leaving the institute were, unfortunately, not always that well-defined. Most patients were individually judged by their psychiatrist as being able to deal with the challenges outside the clinical setting. Other patients were selected because the policy of the psychiatric institute was to close some of the long-stay wards. The depressed group consisted of patients who had just started 
treatment with antidepressants. For more details of the subject characteristics see Table 2. All participants provided their written and informed consent. It has to be noted that the future outpatients were significantly younger than the inpatients and the controls. Furthermore, the distribution of male and female participants differed across groups. Whereas male patients dominated the schizophrenia group, the depression group mainly consisted of female participants. Finally, the level of education differed significantly between groups. Particularly the depressed patients had lower levels of education.

Table 2: Subject characteristics.

\begin{tabular}{|c|c|c|c|c|c|c|c|}
\hline \multirow[t]{2}{*}{ Group } & \multicolumn{2}{|c|}{ Schizophrenia } & \multirow{2}{*}{$\begin{array}{l}\text { Depression } \\
(\mathrm{n}=30)\end{array}$} & \multirow{2}{*}{$\begin{array}{l}\text { Controls } \\
(\mathrm{n}=42)\end{array}$} & \multirow{2}{*}{$\begin{array}{l}\text { F } \\
(3,136)\end{array}$} & \multirow[t]{2}{*}{$\mathrm{P}$} & \multirow{2}{*}{$\begin{array}{l}\text { Group } \\
\text { contrasts }\end{array}$} \\
\hline & $\begin{array}{l}\text { Outpatients } \\
(\mathrm{n}=30)\end{array}$ & $\begin{array}{l}\text { Inpatients } \\
(\mathrm{n}=34)\end{array}$ & & & & & \\
\hline Mean age & $33.8(9.2)$ & $43.0(9.8)$ & $40.6(11.1)$ & $41.1(11.0)$ & 4.80 & 0.003 & $1<2,4^{\mathrm{a}}$ \\
\hline $\operatorname{Sex}(M / F)$ & $27 / 3$ & $22 / 12$ & $8 / 22$ & $18 / 22$ & $28.39^{\mathrm{b}}$ & 0.001 & - \\
\hline $\begin{array}{l}\text { Median level of } \\
\text { education }^{c}\end{array}$ & 5 & 5 & 4 & 5 & $11.02^{\mathrm{b}}$ & 0.01 & - \\
\hline
\end{tabular}

\section{Clinical assessment}

In the group of patients with schizophrenia, the Positive and Negative Syndrome Scale (PANSS; Kay, Fizsbein \& Opler, 1987) was employed to assess symptoms of schizophrenia. The scale consists of 30 symptom items divided into positive symptoms ( 7 items), negative symptoms (7 items) and general psychopathology (16 items). Each item is rated along a 1-to-7-point severity index, completed on the basis of a semi-structured interview. In addition, sum scores are computed for each of the three subscales. However, the symptomatology of schizophrenia might be better characterized by three, rather than two (i.e. positive and negative) dimensions (Liddle, 1987): positive symptoms, negative symptoms and disorganization symptoms. Therefore, to determine the correlation between psychomotor slowing and clinical symptoms we applied the sum scores of these three clusters of symptoms (see Cameron et al., 2002). The positive-symptom cluster consisted of the following PANSS items: delusions, hallucinations, grandiosity, suspiciousness, and unusual thought content. The negative-symptom cluster contained the following items: blunted affect, emotional withdrawal, poor rapport, passive social withdrawal, lack of spontaneity and flow of conservation, motor retardation, and active social avoidance. The disorganization cluster comprised the items conceptual disorganization, difficulty in abstract thinking, disorientation, and poor attention.

The severity of the depressive symptoms in these patients was judged on the basis of item 6 of the general psychopathology scale, but was also assessed in more detail by means of the Calgary Depression Scale for Schizophrenics (CDSS; Addington, Addington \& Schissel, 1990; Addington et al., 1992). The CDSS is composed of nine items rated on a 4-point scale. In the depression group, 


\section{4 | Chapter 6}

the severity of the depressive symptoms was established by the Hamilton Depression Rating Scale (HDRS; Hamilton, 1960).

\section{Apparatus, tasks and procedure}

Drawing movements were recorded by means of a digitizing writing tablet (WACOM 1218RE) and a special pressure-sensitive pen.

\section{Figure-copying task: 'non-conflicting' and 'conflicting' patterns}

This copying task consisted of patterns in which the graphic production rules could be easily applied ('non-conflicting patterns'; NC) or patterns in which two or more of these rules were in conflict with each other ('conflicting patterns'; C). We refer to Figure 1 for the subset of threesegment patterns that were analyzed in the present study. The original task consisted of $12 \mathrm{NC}$ and $\mathrm{C}$ pairs, also including two-segment patterns. The stimuli to be copied were presented on a computer screen. The participants were instructed to copy these patterns on a normal sheet of paper that was placed on the writing tablet. Preprinted rectangles ( 30 by $40 \mathrm{~mm}$ ) indicated the place where the patterns had to be drawn. Before each trial, the participants had to place the pen in a small circle located at the lower left corner of the rectangle. While holding the pen in this position for a random waiting period (ranging between 500 and $2000 \mathrm{~ms}$ ), the stimulus appeared on the screen accompanied by a warning signal $(1000 \mathrm{~Hz}, 300 \mathrm{~ms})$. The participants were asked to copy the stimulus as fast and as accurately as possible. As soon as the subject started drawing the stimulus disappeared from the screen. After they had completed drawing the pattern, they were instructed to place the pen in a circle at the upper right corner of the drawing box for at least $300 \mathrm{~ms}$, which was affirmed by a low tone of $500 \mathrm{~Hz}$.

\section{Fitts' task: moving to a target}

In contrast to the earlier studies, the patient groups in the present study performed an additional psychomotor task. They were instructed to move the pen as fast as possible from a black dot (diameter $0.2 \mathrm{~cm}$ ) towards a gray-colored target (two target widths: 0.375 and $1.5 \mathrm{~cm}$ ) both presented on normal sheet of paper. The distance between the middle of the black dot and the middle of the gray circles was $3 \mathrm{~cm}$. The task consisted of 24 trials, of which the first four were used for practice. The patients had to keep the pen stationary in the black dot for a (random) period lasting between 500 and $2000 \mathrm{~ms}$. After hearing a beep $(1500 \mathrm{~Hz}, 100 \mathrm{~ms})$, they had to move the pen towards the target as fast as possible. As soon as the pen had reached the center of the target, a low beep $(500 \mathrm{~Hz}, 500 \mathrm{~ms})$ was sounded to indicate that the trial had been successfully completed.

This task can be viewed as a variant of tasks following Fitts' law (Fitts, 1954). This law describes the relationship between the duration of the movement (MT) and the conditions of the movement, specifically the amplitude of the movement and the target width. 

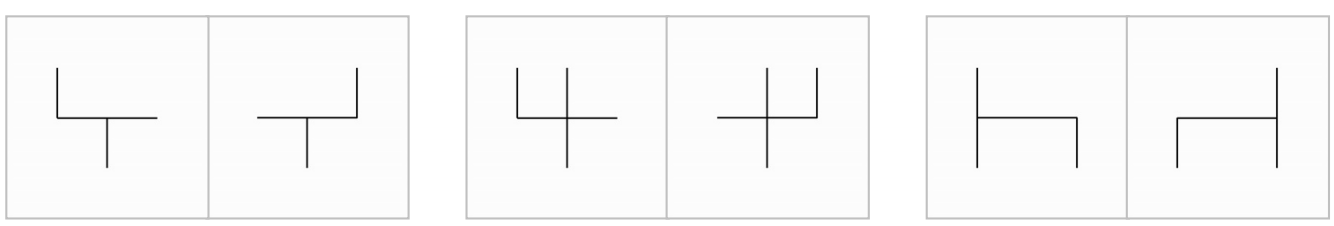

Figure 1: The three pairs of patterns used in the copying task of the present study showing the nonconflicting patterns $(\mathrm{NC})$ on the left-hand side and the conflicting patterns $(\mathrm{C})$ on the right-hand side.

\section{Digit Symbol Test (DST)}

The Dutch version of the Digit Symbol Test (DST), which is part of the WAIS (Wechsler, 1956), was administered to the patient groups only. In this test, participants are presented with a sheet of paper depicting several rows of boxes containing a variety of symbols. Below each symbol there is an empty box. Subjects were asked to fill in the corresponding digits as fast as possible according to a key consisting of nine symbol-digit pairs that were displayed at the top of the sheet. The time limit for completing the task was 90 seconds.

\section{Recording and analysis}

During the execution of the tasks, the $\mathrm{x}$ and y positions of the pen on, and $5 \mathrm{~mm}$ above, the digitizer were recorded with a frequency of $200 \mathrm{~Hz}$ and a spatial precision of $0.2 \mathrm{~mm}$. Based on pen pressure (the threshold was assessed for each trial separately, but ranged around $0.24 \mathrm{~N}$ ), movement trajectories were defined as either pen-up periods or pen-down periods.

The following variables were calculated for the figure-copying task and the Fitts' task. Initiation time (IT), defined as the pen-up period (ms) between the 'go' signal (in the copying task accompanied by the appearance of the stimulus) and the start of the first pen-down movement on the paper. The succeeding execution period, defined as the time between the start of the first and the completion of the last drawing movement on the paper, was divided into movement time (MT), i.e. the accumulated pen-down periods, and pen-up time (UpT), i.e. the accumulated pen-up periods. The latter variable was only calculated for the figure-copying task.

In the figure-copying task, we also examined for each individual pattern whether subjects had adhered to one of the main graphic production rules, the so-called threading rule, i.e. connecting successive strokes without interposed pen lifts. This rule was studied only for those segments of the pattern that could actually be threaded, i.e. the L-shape in the different orientations. The adherence score could be either 0 (no adherence to the rule) or 1 (adherence to the rule). Subsequently, we calculated the mean adherence scores for both the NC and C patterns.

For the DST, we calculated the raw score, i.e. the number of digits that had been filled in correctly after 90 seconds, matching time as the accumulated pen-up periods between two successive digits and the pause intervals between successive digits, and writing time, defined as the time needed to produce the entire digit. Matching time and writing time were calculated as a mean 
value per digit. In this latter calculation, the first digit of each new line was ignored because of the large distance that had to be covered by the pen.

Data were analyzed by means of SPSS for Windows. For the figure-copying task, statistical evaluation was performed for each movement variable with analysis of variance (GLM for repeated measures) with Group as a between-subject factor and 'NC versus C' as a within-subject factor. Group differences were further analyzed by means of a Bonferroni test. Within each group, pairedsamples $t$-tests were applied to analyze the differences between the NC and $\mathrm{C}$ patterns for the obtained variables.

Differences in the mean adherence scores between the groups were analyzed by means of a Kruskal-Wallis Test. For the Fitts' task and the DST, differences between patient groups were computed by means of a one-way ANOVA. Correlations between the performances on the figurecopying and psychomotor speed tasks (Fitts' task and DST) and the clinical rating scores were calculated by means of Spearman's $\rho$.

\section{Results}

\section{Clinical assessment}

Contrary to our expectations, the schizophrenia inpatients did not show more severe levels of psychopathology when compared to the outpatients (see Table 3). The scores on the PANSS were not significantly different between the two subgroups. Obviously, the severity of psychopathology was not an important selection criterion to determine whether patients could be placed in an outside residence (the psychiatrists involved were, of course, not aware of the PANSS scores). The results of the depression score of the PANSS and the CDSS show that the inpatients also did not display more severe depressive symptoms than the outpatients. The mean score on the CDSS was even slightly higher in the outpatients subgroup.

Table 3: Mean values and standard deviations (between brackets) of psychopathology scores and their corresponding statistics for the two schizophrenia subgroups.

\begin{tabular}{lcccc}
\hline Group & $\begin{array}{l}\text { Outpatients } \\
(\mathrm{n}=30)\end{array}$ & $\begin{array}{l}\text { Inpatients } \\
(\mathrm{n}=34)\end{array}$ & $\mathrm{F}(1,63)$ & $\mathrm{P}$ \\
\cline { 2 - 4 } PANSS & $13.3(4.1)$ & $12.3(3.6)$ & 1.15 & 0.29 \\
Positive symptoms subscale & $17.2(7.5)$ & $15.8(6.8)$ & 0.59 & 0.44 \\
Negative symptoms subscale & $32.9(9.9)$ & $32.9(8.1)$ & 0.00 & 0.99 \\
General psychopathology subscale & $2.5(1.3)$ & $2.4(1.1)$ & 0.16 & 0.69 \\
Depression score (item g6) & $\mathrm{a}$ & $10.1(3.6)$ & 1.23 & 0.27 \\
Cluster of positive symptoms & $11.1(3.8)$ & $16.7(7.2)$ & 0.13 & 0.72 \\
Cluster of negative symptoms & $17.4(7.7)$ & $8.8(3.7)$ & 0.09 & 0.77 \\
Cluster of disorganization symptoms & $8.5(4.3)$ & $3.4(3.9)$ & 0.85 & 0.36 \\
CDSS & $4.3(4.0)$ & & & \\
\hline
\end{tabular}

${ }^{\mathrm{a}}$ item $\mathrm{g} 6=$ item 6 of the general psychopathology scale. 
The mean score of the Hamilton Depression Scale in the depressed patients was $26.6(\mathrm{SD}=6.4)$, which indicates that they exhibited severe features of depression (above the cut-off score of 25).

Figure-copying task: 'non-conflicting' and 'conflicting' patterns

\section{Kinematics}

The mean values of initiation time (IT), pen-up time (UpT) and movement time (MT) are displayed in Figure 2.

The results of the GLM for repeated measurements showed a significant main effect of Group on IT $(\mathrm{F}(3,130)=3.77, \mathrm{p}<0.05)$. Post-hoc evaluation showed that this effect was mainly due to a marked difference in IT between the depressed patients and the controls $(p<0.05)$. The two subgroups of patients with schizophrenia performed at a level that was intermediate to the performance levels of the depressed patients and the control subjects, and the two subgroups demonstrated a more or less similar IT. As was to be expected, IT was significantly longer in copying $\mathrm{C}$ patterns than in copying NC patterns $(\mathrm{F}(1,130)=4.49, \mathrm{p}<0.05)$. This effect was not found to be different for the four groups: the interaction between Group by NC versus $\mathrm{C}$ was not significant $(\mathrm{F}(3,130)=1.74, \mathrm{p}=0.16)$. The results of the paired-samples $t$-tests revealed that only the depressed patients showed a significant difference in IT between the $\mathrm{NC}$ and $\mathrm{C}$ patterns $(t(28)=$ $-2.93, \mathrm{p}<0.01)$. Both the inpatients and outpatients subgroups did not demonstrate a prolongation of IT when starting rules conflicted.

Groups also differed with respect to the mean duration of $\operatorname{UpT}(\mathrm{F}(3,130)=3.00, \mathrm{p}<0.05)$. Post-hoc analyses of this group effect, however, revealed that only the difference between the depressed patients and controls was apparent, though not significant $(\mathrm{p}=0.06)$. As expected, copying $\mathrm{C}$ patterns resulted in significantly longer hesitations above the paper when compared to the NC patterns $(\mathrm{F}(1,130)=32.52, \mathrm{p}<0.001)$. This effect did differ clearly across groups (Group X $\mathrm{NC}$ versus $\mathrm{C}: \mathrm{F}(3,130)=5.21, \mathrm{p}<0.01)$. The UpT's of the subgroup of inpatients with schizophrenia now very closely resembled those of the depressed patients. In both these groups, the results of the paired-samples $t$-tests showed a pronounced, significant difference $(\mathrm{p}<0.001)$ in UpT between the $\mathrm{NC}$ and $\mathrm{C}$ patterns. By contrast, in the subgroup of outpatients no such difference could be observed. Post-hoc analyses showed that this smaller difference in UpT between NC and C in the outpatients differed significantly $(p<0.05)$ when compared to the inpatients and the depressed patients; the difference with the controls approached significance $(\mathrm{p}=0.08)$.

Similar to IT and UpT, MT differed significantly across groups $(F(3,130)=4.94), p<0.01)$. Post-hoc analyses showed that this contrast was again greatest between the depressed patients and the controls $(p<0.01)$. Nevertheless, there was also a strong tendency $(p=0.06)$ for the schizophrenia inpatients to exhibit longer MT's when compared to the controls. Although conflicts between rules are expected to predominantly affect IT and UpT, hesitations may also occur during the actual drawing movements. Indeed, MT was also significantly affected during the production of the conflicting patterns $(\mathrm{F}(1,130)=17.27, \mathrm{p}<0.001)$. Whereas the histogram in Figure 2 representing the mean values of MT for NC and C suggests a similar profile of MT to that of UpT, 


\section{8 | Chapter 6}

the interaction between Group by $\mathrm{NC}$ versus $\mathrm{C}$ was now not significant (Group $\mathrm{X} \mathrm{NC}$ versus C: $\mathrm{F}(3,130)=1.86, \mathrm{p}=0.14)$.
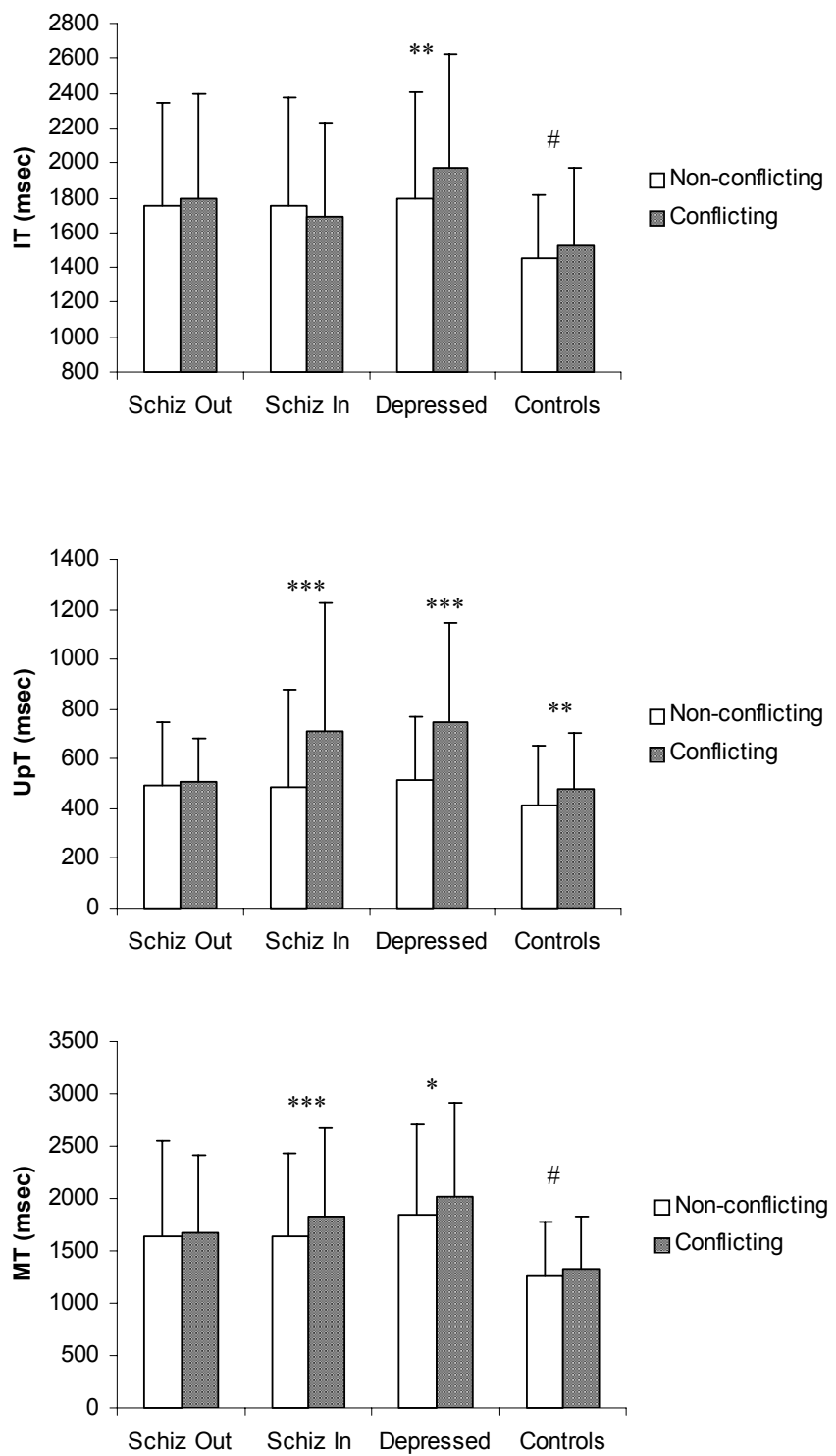

Figure 2: Mean values of initiation time (IT), pen-up time (UpT) and movement time (MT) for the four different groups in the figure-copying task (Schiz Out $=$ Schizophrenia outpatients, Schiz In $=$ Schizophrenia inpatients, Dep $=$ Depressed patients). ${ }^{* *} \mathrm{p}<0.001,{ }^{* *} \mathrm{p}<0.01,{ }^{*} \mathrm{p}<0.05, \# \mathrm{p}<0.10$ 


\section{Adherence to the threading rule}

From the above, it can be concluded that, except for the outpatients, conflicting rules had the greatest effect on UpT. Higher values of UpT imply that the subject lifts the pen more often from the paper and/or holds the pen above the writing surface for a longer period of time. The normal preference to keep the pen on the paper is captured by the so-called threading rule.

The mean adherence scores for the threading rule are shown in Figure 3. Not surprisingly, almost all subjects threaded during the copying of the NC patterns, whereas in the $\mathrm{C}$ condition, subjects adhered less often to this rule. Most importantly, the application of the threading rule tended to differ between groups $\chi^{2}(3, \mathrm{n}=136, \mathrm{p}=0.07)$. As might be expected from the results of UpT, the schizophrenia outpatients adhered more strongly to the threading rule, whereas the depressed patients adopted this rule less frequently.

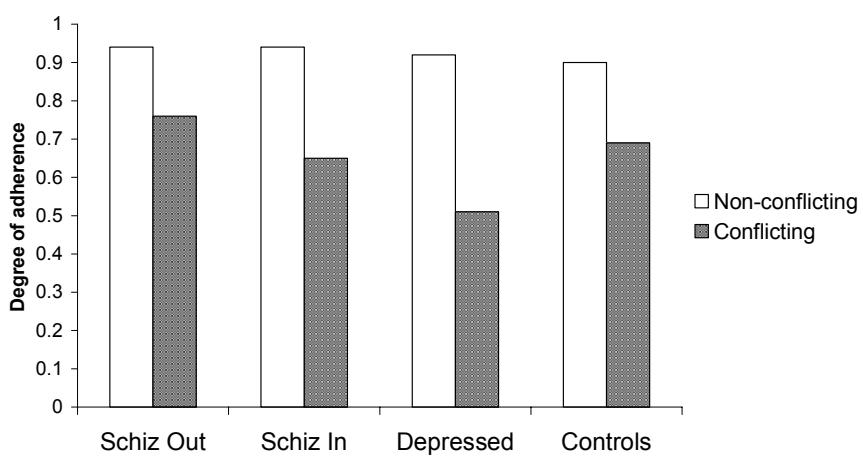

Figure 3: Mean adherence scores for the threading rule for the four groups (Schiz Out $=$ Schizophrenia outpatients, Schiz In = Schizophrenia inpatients, Dep = Depressed patients).

\section{Fitts' task: moving to a target}

The mean values of initiation time (IT) and movement time (MT) are displayed in Table 4. IT differed significantly between the patient groups. Further exploration of this difference showed that the schizophrenia future outpatients were significantly faster in the initiation of their movements when compared to the depressed patients. No significant differences between the patient groups existed for the time needed to move towards the target. 
Table 4: Mean values of the variables and their corresponding statistics in the Fitts' task and the DST for all patient groups.

\begin{tabular}{lccccccccl}
\hline & \multicolumn{2}{l}{ Schizophrenia Out } & \multicolumn{2}{l}{ Schizophrenia In } & \multicolumn{2}{l}{ Depressed } & F $(2,80)$ & P & Group \\
& Mean & (SD) & Mean & (SD) & Mean & (SD) & & & contrasts \\
\hline Fitts' task & & & & & & & & & \\
IT (ms) & 342 & $(102)$ & 389 & $(91)$ & 446 & $(203)$ & 3.50 & 0.04 & $1<3^{\text {a }}$ \\
MT (ms) & 608 & $(268)$ & 725 & $(278)$ & 790 & $(473)$ & 1.73 & 0.18 & ns \\
DST & & & & & & & & & \\
Raw score (n) & 39.7 & $(13.1)$ & 33.9 & $(13.2)$ & 41.5 & $(15.3)$ & 2.61 & 0.08 & ns \\
MatchT (ms) & 2045 & $(813)$ & 2363 & $(1062)$ & 1922 & $(1064)$ & 1.64 & 0.20 & ns \\
WriteT (ms) & 402 & $(82)$ & 546 & $(242)$ & 526 & $(151)$ & 5.55 & 0.01 & $1<2,1<3$ \\
\hline
\end{tabular}

a 1 Schizophrenia Out, 2 Schizophrenia In, 3 Depressed; ns = non-significant.

$D S T$

The mean values of the raw score, matching time and writing time are displayed in Table 4 . There was a tendency $(p=0.08)$ for the raw score to differ between the patient groups. No significant difference between the patient groups was found for matching time. Writing time, however, proved to be significantly different across the patient groups. Post-hoc analyses demonstrated that the schizophrenia future outpatients were significantly faster in writing the digits than both the schizophrenia inpatients and the depressed patients.

\section{Correlations between figure-copying, psychomotor speed, and clinical symptoms}

To examine the relationship between the performances on the figure-copying task and psychomotor speed, as assessed by means of the Fitts' task and the DST, correlation coefficients were calculated (see Table 5). Within the schizophrenia group, not surprisingly, most of the movement variables extracted from the Fitts' task and the DST correlated significantly with the mean values of IT, UpT and MT in the figure-copying task. The most interesting finding was that greater discrepancies in IT, UpT and MT between the NC and C condition were significantly correlated with longer MT's in the Fitts' task. In the DST, the duration of writing time was also found to be related to larger differences in UpT between $\mathrm{NC}$ and $\mathrm{C}$.

There was only one significant correlation between psychomotor speed and psychopathology: patients with higher scores on cognitive disorganization showed longer matching times on the DST. Contrary to other recent findings (Brébion et al., 2000; Holthausen et al., 1999), we did not find significant correlations between psychomotor slowing and depressive symptoms. 
Table 5: Correlations between the movement variables of the figure-copying task, the clinical ratings, and the patients' psychomotor speed (Fitts' task and the DST) for the schizophrenia group as a whole.

\begin{tabular}{lllll}
\hline \multicolumn{1}{l}{ Fitts' task } & \multicolumn{3}{l}{ DST } \\
\hline $\begin{array}{l}\text { Figure-copying task } \\
\text { Mean values }\end{array}$ & IT & MT & MatchT & Write T \\
$\quad$ IT & 0.26 & 0.24 & $0.36^{* *}$ & $0.42^{* *}$ \\
UpT & $0.28^{*}$ & $0.48^{* *}$ & $0.40^{* *}$ & $0.65^{* *}$ \\
MT & 0.26 & $0.48^{* *}$ & 0.25 & $0.52^{* *}$ \\
Differences between NC and C & & & & \\
IT & 0.23 & $0.42^{* *}$ & 0.02 & 0.21 \\
UpT & 0.15 & $0.28^{*}$ & 0.25 & $0.28^{*}$ \\
MT & 0.14 & $0.32^{*}$ & 0.13 & 0.12 \\
PANSS & & & & \\
Positive symptoms cluster & -0.04 & -0.04 & 0.15 & -0.06 \\
Negative symptoms cluster & 0.03 & 0.14 & 0.21 & -0.01 \\
Disorganization symptoms cluster & -0.01 & 0.02 & $0.50^{* *}$ & 0.10 \\
Depression score (item g6) & & & & \\
CDSS & 0.07 & -0.01 & 0.07 & 0.02 \\
\hline
\end{tabular}

a item g6 $=$ item 6 of the general psychopathology scale. ${ }^{* *} \mathrm{p}<0.01,{ }^{*} \mathrm{p}<0.05$

\section{Discussion}

The present study was aimed at elucidating differences in planning behavior between patient groups on a figure-copying task. Of particular concern were possible differences between subgroups of patients with schizophrenia. We previously reported that several groups of schizophrenia inpatients performed more similarly to a group of depressed patients than to a group of (future) outpatients (Hulstijn et al., 2001). The inpatients and the depressed group showed particularly prolonged hesitations above the paper during the copying of so-called 'conflicting' (C) patterns when compared to 'non-conflicting' (NC) patterns, which was not the case for the subgroup of outpatients. The goal of the present study was to clarify why some patients with schizophrenia, in our case patients scheduled to be released from the institute (the future outpatients), were less affected by the planning constraints induced by conflicting rules than inpatients who would remain hospitalized. For this purpose, we described the results of a direct comparison between these two subgroups.

The first question to be answered was whether differences between inpatients and (future) outpatients could be explained by differences in the severity of psychopathology and particularly to differences in depressive features. Contrary to expectations, the results of the present study showed that these subgroups of patients with schizophrenia did not differ with respect to PANSS scores and depression scores. Thus, the finding that the inpatients exhibited larger differences between the nonconflicting (NC) and conflicting (C) patterns could not be attributed to a more severe psychopathology or to the number or severity of depressive features. 
The second question was whether differences in the performance on the figure-copying task could be attributed to a general psychomotor slowing characteristic of schizophrenia, and particularly depressive symptoms. This slowing was assessed by two psychomotor tasks, i.e. the DST and a goal-directed line-drawing task (a so-called Fitts' task). The results showed that, overall, in all patients with schizophrenia the differences between $\mathrm{NC}$ and $\mathrm{C}$ in initiation time (IT), pen-up time (UpT) and movement time (MT) correlated significantly with MT in the Fitts' task. Another significant correlation was found between the difference in UpT and writing time in the DST. Thus, patients with schizophrenia demonstrating a stronger psychomotor slowing also showed a greater discrepancy between copying either NC or C patterns. This pattern of results resembled that of the depression group. Yet, the hypothesis that some patients with schizophrenia might display a depressive-like psychomotor slowing (c.f. Brébion et al., 2000) could not be corroborated since we did not find significant correlations between our measures of psychomotor speed and the clinical ratings of depressive symptoms (CDSS scores and the PANSS depression item). It needs to be noted that Brébrion et al. (2000) used a different scale, i.e. the Hamilton Depression Rating Scale, which is particularly designed to measure vital signs of major depression, such as sleeping problems, retardation, and weight loss, but might be less appropriate to reflect distinct depressive symptoms in schizophrenia. Also Holthausen et al. (1999) found a significant relationship between depression scores (based on a dimensional sum score of four PANSS items) and psychomotor speed. But again, their measures of psychomotor speed (Stroop test, Trailmaking Test and fingertapping) were different from those used in our study. Another complicating factor is that not all depressed patients are slowed; some patients are even rather agitated. To conclude, we share Brébion et al.'s (2000) view that, although psychomotor slowness and depression might be linked in at least a number of patients, they may also behave as independent factors. The attribution of an underlying psychomotor slowing needs further exploration. Moreover, the significant correlation that was found between matching time in DST performance and symptoms of cognitive disorganization illustrates that the DST does not only measure perceptual-motor speed, but also several other cognitive processes like visual-scanning efficiency and memory (c.f. Joy, Fein \& Kaplan, 2003). Patients with more severe features of cognitive disorganization are likely to lack some the cognitive efficiency that is necessary for optimal performance in the DST.

It needs to be noted that the results of the present study have to be interpreted with a certain amount of caution. First, the subgroups of patients with schizophrenia we studied were not selected at random and the criteria for being assigned to either of the two subgroups were not always that strict. Second, the subgroups differed with respect to age. The surprising finding that the schizophrenia outpatients were faster on some movement aspects may perhaps be partially explained by the fact that they were younger than the inpatients. However, since we lack age-related data, this explanation is speculative.

What do the present findings tell us about planning difficulties in schizophrenia? Taken collectively, our findings suggest that patients with schizophrenia may differ in planning ability from, for example, depressive patients. Patients with schizophrenia who do not show features of psychomotor slowing seem to rely more strongly on a routine or automatic strategy when graphic rules conflict with each other: they simply follow the most dominant rule. When, on the other hand, 
patients demonstrate clear signs of psychomotor slowness, they are likely to experience these planning conflicts to a similar extent as depressed patients do. Moreover, the current results also imply that planning problems in patients with schizophrenia may have very distinct consequences for the individual patient's behavior, which is in line with the theory of Frith (1992). Some patients are able to choose between alternatives, although they require a considerable amount of time to do so. Other patients, by contrast, may lack this ability, which entails that they react in a less flexible manner on the varying task demands and revert to an automatic or stereotyped response pattern, as shown by our current findings. Another possible reaction pattern is that, because of this failure, certain patients are less able to inhibit their immediate responses to external stimuli or cues, which was shown by one of our most recent studies (Jogems-Kosterman et al., accepted in a shortened and revised version, see the succeeding Chapter). In this study, patients with schizophrenia were asked to copy L-shaped patterns in which the point from which the subject had to start drawing was imposed (indicated by a black dot in the pattern itself). It appeared that a number of patients, particularly those with clinical symptoms of cognitive disorganization, were inclined to react faster on these extra external cues, irrespective of whether this cue corresponded to or opposed their normal preference.

Our final remark concerns the overall implications of the present study. To what extent are the results of the present study in conflict with earlier findings that have been reported? Our analyses showed that psychomotor slowing might be an important aspect that can explain differences in planning performances. However, although impaired planning ability is an important feature of schizophrenia, it may very well be the product of different underlying mechanisms. How these planning deficits relate to underlying cognitive processes, on the one hand, and the overt clinical features of the illness, on the other, deserves further clarification. In this perspective, we believe that future investigations that are based on the type of tasks as used in the present study will contribute to a deeper understanding of the planning problems observed in schizophrenia. It is of particular relevance to explore the consequences of the planning deficiencies in patients with schizophrenia for the functional outcome and successful outplacement of these patients (Fakkers et al., 2002). 
104 | Chapter 6

\section{References}

Addington, D., Addington, J., \& Schissel, B. (1990). A depressed rating scale for schizophrenics. Schizophrenia Research, 3, 247-251.

Addington, D., Addington, J., Maticka-Tyndale, E., \& Joyce, J. (1992). Reliability and validity of a depression rating scale for schizophrenics. Schizophrenia Research, 6, 201-208.

American Psychiatric Association (1987). Diagnostic and statistical manual of mental disorders (3rd edition revised).Washington, D.C.: APA.

Benson, F. (1990) Psychomotor retardation. Neuropsychiatry, Neuropsychology and Behavioral Neurology, 3, 36-47.

Bermanzohn, P.C., \& Siris, S.G. (1992). Akinesia: A syndrome common to Parkinsonism, retarded depression, and negative symptoms of schizophrenia. Comprehensive Psychiatry, 33, 221-232.

Brébion, G., Amador, X., Smith, M., Malaspina, D., Sharif, Z., \& Gorman, J.M. (2000). Depression, psychomotor retardation, negative symptoms, and memory in schizophrenia. Neuropsychiatry, Neuropsychology and Behavioral Neurology, 13, 177-183.

Cameron, A.M., Oram, J., Geffen, G.M., Kavanagh, D.J., McGrath, J.J., \& Geffen, L.B. (2002). Working memory correlates of three symptom clusters in schizophrenia. Psychiatry Research, 110, 49-61.

Crow, T.J. (1980). Molecular pathology of schizophrenia: More than one disease process? British Medical Journal, 280, 66-68.

Elliott, R., Baker, S.C., Rogers, R.D., O’Leary, D.A., Paykel, E.S., Frith, C.D., Dolan, R.J., \& Sahakian, B.J. (1997). Prefrontal dysfunction in depressed patients performing a complex planning task: A study using positron emission tomography. Psychological Medicine, 27, 931-942.

Fakkers, G.P.F.M., Jogems-Kosterman, B.J.M., Loonen, A.J.M., Van Hoof, J.J.M., \& Hulstijn, W. (2002). Changes in clinical functioning and neurocognitive performances in patients with schizophrenia during the first years of deinstitutionalization. Schizophrenia Research, 53, 127.

Frith, C.D. (1992). The cognitive neuropsychology of schizophrenia. Hove: Lawrence Erlbaum Associates.

Gallhofer, B., Bauer, U., Lis, S., Krieger, S., \& Gruppe, H. (1996). Cognitive dysfunction in schizophrenia: Comparison of treatment with atypical antipsychotic agents and conventional neuroleptic drugs. European Neuropsychopharmacology, 6, (S2),13-20.

Hamilton, M. (1960). A rating scale for depression. Journal of Neurology, Neurosurgery and Psychiatry, 23, 56-62.

Hanes, K.R., Andrewes, D.G., Pantelis, C., \& Chiu, E. (1996). Subcortical dysfunction in schizophrenia: A comparison with Parkinson's Disease and Huntington's Disease. Schizophrenia Research, 19, 121-128.

Holthausen, E.A.E., Wiersma, D., Knegtering, R.H., \& Van den Bosch, R.J. (1999). Psychopathology and cognition in schizophrenia spectrum disorders: The role of depressive symptoms. Schizophrenia Research, 39, 65-71.

Hulstijn, W., Jogems-Kosterman, B.J.M., Wezenberg, E., \& Sabbe, B.G.C. (2001). An evaluation of the use of figure-copying tasks in studies of planning deficits in schizophrenia. In: R.G.J. Meulenbroek \& B. Steenbergen (Eds.), Proceedings of the tenth biennial conference of the International Graphonomics Society (pp. 46-51). Nijmegen: IGS. 
Jogems-Kosterman, B.J.M., Hulstijn, W., Van Hoof, J.J.M., \& Thomassen, A.J.W.M. (1999). Is the planning of action more impaired in schizophrenic patients than in depressed patients? The effects of conflicting graphic production rules. In: G. Leedham, M. Leung, V. Sagar \& X. Xuhong (Eds.), Proceedings of the 9th biennial conference of the International Graphonomics Society (pp. 225-229). Singapore: NTU.

Jogems-Kosterman, B.J.M., Zitman, F.G., Van Hoof, J.J.M., Hulstijn, W. (2001). Psychomotor slowing and planning deficits in schizophrenia. Schizophrenia Research, 48, 317-333.

Jogems-Kosterman, B.J.M., Hulstijn, W., Wezenberg, E., \& Van Hoof, J.J.M. (accepted in a shortened and revised version). Movement planning deficits in schizophrenia: Failure to inhibit automatic response tendencies. Cognitive Neuropsychiatry.

Joy, S., Fein, D., \& Kaplan, E. (2003). Decoding Digit Symbol: Speed, memory, and visual scanning. Assessment, 10, 56-65.

Kay, S.R., Fizsbein, A., \& Opler, L.E. (1987). The Positive and Negative Syndrome Scale (PANSS) for schizophrenia. Schizophrenia Bulletin, 13, 261-275.

Liddle, P.F. (1987). Schizophrenia syndrome, cognitive performance and neurological dysfunction. Psychological Medicine, 17, 49-57.

Marczewski, P., Van der Linden, M., \& Larøi, F. (2001). Further investigation of the Supervisory Attentional System in schizophrenia: Planning, inhibition, and rule abstraction. Cognitive Neuropsychiatry, 6, 175192.

Morris, R.G., Rushe, T., Woodruffe, P.W.R., \& Murray, R.M. (1995). Problem solving in schizophrenia: A specific deficit in planning ability. Schizophrenia Research, 14, 235-246.

Pantelis, C., Barnes, T.R.E., Nelson, H.E., Tanner, S., Weatherley, L., Owen, A.M., \& Robbins, T.W. (997). Frontal-striatal cognitive deficits in patients with chronic schizophrenia. Brain, 120, 1823-1843.

Shallice, T. (1982). Specific impairments of planning. Philosophical transactions of the Royal Society of London series B Biological sciences, 298, 199-209.

Thomassen, A.J.W.M., \& Tibosch, H.J.C.M. (1991). A quantitative model of graphic production. In: G.E. Stelmach \& J. Requin (Eds.), Tutorials in motor neuroscience (pp. 269-282). Dordrecht: Kluwer Academic Publishers.

Thomassen, A.J.W.M., Meulenbroek, R.G.J., \& Tibosch, H.J.C.M. (1991). Latencies and kinematics reflect graphic production rules. Human Movement Science, 10, 271-289.

Wechsler, D. (1956). Manual for the Wechsler Adult Intelligence Scale. New York: Psychological corporation.

Widlöcher, D., \& Hardy-Bayle, M.C. (1989). Cognition and control of action in psychopathology. European Bulletin of Cognitive Psychology, 9, 583-615. 


\title{
Chapter 7
}

\section{Movement planning deficits in schizophrenia: Failure to inhibit automatic response tendencies ${ }^{1}$}

\begin{abstract}
Explicit planning deficits have been reported frequently in patients with schizophrenia. This study addressed the question whether these patients would already encounter difficulties in a more 'implicit' process of planning an optimal sequence of movements in simple actions. Twenty patients with schizophrenia and 20 controls participated in the study. The motor tasks consisted of copying simple line drawings in which the planning of a movement sequence was taxed along various dimensions. In the first task the number of line elements varied between one and two, and the two-line figures differed with respect to the ease with which the so-called graphic production rules could be applied. In the second task the spatial position of the drawing varied between an easy center position and a somewhat more difficult corner location. In the third task subjects were asked to start copying either at a preferred or at a less preferred starting point. The results showed that the patients did not encounter planning difficulties while copying the one- or two-segment patterns. However, the variations in the spatial conditions and starting point did affect the patients' performance more than that of the controls. Whereas the patients with higher negative symptom scores tended to be slower when they were forced to start drawing at a particular point, the patients predominantly exhibiting features of disorganization proved to be faster in this condition. The findings suggest a fairly intact initiation of simple movement sequences in patients with schizophrenia, but difficulties in inhibiting automatic response tendencies. It is concluded that this inhibition failure may play an important role in the planning difficulties these patients experience.
\end{abstract}

\footnotetext{
${ }^{1}$ Based on: Jogems-Kosterman, B.J.M., Hulstijn, W., Wezenberg, E., \& Van Hoof, J.J.M. (accepted in a shortened and revised version). Cognitive Neuropsychiatry.
} 


\section{Introduction}

The ability to plan actions is essential for daily life. Many patients with schizophrenia, however, appear to encounter planning difficulties. They are not able to arrange their activities throughout the day in an orderly way. Problems with the generation and planning of actions are thought to be primary features of schizophrenia (Frith, 1992; Widlöcher \& Hardy-Bayle, 1989). This deficiency may be expressed by a lack of initiative in performing actions (poverty of action or psychomotor slowing); it may also result in stereotypical behavior (repeating actions purposelessly) or in chaotic or disorganized behavior (Frith, 1992). In order to understand the nature of these daily problems, it is of great importance to unravel the underlying cognitive and motor deficits. This will then help psychiatrists and family members, as well as the patients themselves, to gain a better insight into the problems the patients are faced with.

What mental processes are required for planning an action successfully? Duncan (1995) distinguishes three essential components. First, a subject has to identify the goal and develop subgoals. Second, in choosing among subgoals, consequences must be anticipated. Third, the subject has to consider what is needed to achieve the goal. In this process of action planning, it is important to inhibit responding to inappropriate stimuli (i.e., stimulus-driven behavior; Frith, 1992).

Explicit planning deficits in schizophrenia are most frequently investigated by means of the Tower of London (Shallice, 1982). This test requires the planning ahead of a sequence of movements so that the positions of three colored balls correspond to a target condition. Most studies showed impaired performance in patients with schizophrenia (Morris et al., 1995; Hanes et al., 1996; Pantelis et al., 1997; Marczewski, Van der Linden \& Larøi, 2001). However, in a study by Krabbendam et al. (1999) the performance of a group of patients with schizophrenia was not significantly poorer than that of a healthy control group. The reason for this deviating result could be that in the Krabbendam et al. study (1999) the participants' performance on the Tower of London was controlled for general intellectual level. This may touch on an important disadvantage of the use of explicit planning tests: the test measures the 'intellectual' and conscious ability of decomposing the task into subgoals and putting these in the most appropriate order. This type of tests, however, might not capture the actual problems that patients encounter in daily life. Most of our actions are not governed by explicit (and more or less 'intellectual') rules but by implicit rules of which we are not aware. Another difficulty with most tests measuring planning is that several other cognitive processes and, in particular, working memory, are also involved. Consequently, impaired test performances may result from multiple causes, making it difficult to identify specific planning deficits.

Graphic tasks, initially, do not require a conscious explicit planning process. Our writing and drawing movements appear to be governed by certain implicit rules, the so-called graphic production rules (Thomassen \& Tibosch, 1991; Thomassen, Meulenbroek \& Tibosch, 1991). These rules specify preferences with respect to starting points (starting at the leftmost point; starting at the topmost point; starting with a vertical segment), stroke directions (drawing horizontal lines from left to right; drawing vertical lines from top to bottom) and stroke sequences (drawing successive lines without a pen lift; anchoring lines on previously drawn lines; drawing parallel lines in immediate succession). When children learn to draw and write, graphic production rules are gradually built up 
as procedural knowledge (Vinter, 1993). For example, when copying simple figures adult subjects appear to follow these graphic production rules more or less automatically (Thomassen \& Tibosch, 1991). Certain patterns (like the letter L) can be drawn completely in accordance with these rules. However, when they are asked to copy the mirrored image of such figures, subjects will find that these rules are in conflict with the task demands. It has been found that these conflicts affect the speed of copying. In copying 'conflicting' patterns, subjects show somewhat longer initiation times and during execution significantly longer pen-up times, when compared to the copying of 'nonconflicting' patterns (Thomassen, Meulenbroek \& Tibosch, 1991). These findings suggest that in the 'conflicting' condition, subjects take more time to plan and execute their drawing movements.

In our attempt to unravel planning problems in patients with schizophrenia, we recently applied a copying task including both 'non-conflicting' and 'conflicting' patterns in a large sample of patients (Hulstijn et al, 2001; Jogems-Kosterman et al., 1999 and Chapter 5 of this thesis). A great advantage of this type of task is that the figures to be copied are rather simple and, by mirroring the stimuli, the perceptual and memory load is kept constant. As was to be expected from studies showing that schizophrenia is associated with intact procedural learning (Clare et al, 1993; Goldberg, et al, 1993; Watanabe et al., 2002), the results demonstrated that patients with schizophrenia performed within the normal range when they copied the 'non-conflicting' patterns. However, in copying 'conflicting' patterns patients performed differently compared to the healthy controls. Except from a subgroup of future outpatients, patients with schizophrenia displayed significantly longer hesitations above the paper while copying 'conflicting' patterns (Hulstijn et al., 2001; Jogems-Kosterman et al., 1999).

In earlier studies figure-copying tasks were successfully applied to investigate cognitive and motor performance in patients with schizophrenia (Van Hoof et al., 1998; Jogems-Kosterman et al., 1999, 2001) as well as in patients with depression (Van Hoof et al., 1993, 1998; Sabbe et al., 1996a, 1996b). During the execution of these tasks, drawing and writing movements are digitally recorded allowing the temporal and spatial features of these movements to be analyzed accurately. Like most neuropsychological tests, figure-copying tasks involve several cognitive, sensory and motor processes (Van Sommers, 1989; Van Galen, 1991; Hulstijn, 1994). First, perceptual processes are needed for the visual analysis of the stimulus, after which its features and/or global information can be stored in working memory. If the stimulus is, or resembles, a familiar object, a global visual representation may be retrieved from long-term visual memory and/or a verbal label may be retrieved from long-term verbal memory. Second, visual-spatial processes are employed for detailed perception and planning, e.g., of the orientation and relative size of line segments. Subsequently, the movement sequence has to be organized and planned, which does not mean that all required movements have to be planned in advance, but at least the starting point and direction of the first line need to be chosen beforehand. Finally, motor processes are invoked for the preparation, coordination, initiation and execution of the muscle commands needed for drawing, which includes the monitoring of visual feedback to correct any errors. During the latter two stages of the entire process, graphic production rules are likely to be followed in order to facilitate the planning and execution of the movements. 
From the above it can be concluded that poor performance in figure-copying tasks might stem from deficits in any or more than one of the processes involved. In our previous study (Jogems-Kosterman et al., 2001 and Chapter 4 of this thesis), we found that patients with schizophrenia, particularly those with marked negative symptoms, were clearly retarded in their preparation and planning of the drawing movements. Patients also needed longer reinspection times when copying the most complex and unfamiliar figures. We suggested that patients tended to start copying before they had planned all the required movements (c.f. Tower of London findings, Morris et al., 1995; Hanes et al., 1996; Pantelis et al., 1997; Marczewski, Van der Linden \& Larøi, 2001), which may point to an inhibition failure. However, since the planning and copying of complex figures place demands on working memory, another possible explanation for these results was that patients were not able to hold information 'on-line' during task performance.

The aim of the present study was to assess whether patients with schizophrenia would encounter difficulties with (1) the initiation and planning of simple movement sequences (that do not challenge the capacity of working memory), and (2) the inhibition of applying highly preferred graphic production rules. To answer the first question we used a task in which subjects were required to copy stimuli that were either single-line segments or patterns consisting of two lines. The two-line (L-shaped) patterns were either 'non-conflicting' or 'conflicting'. To enhance the planning demands further, in a second task we varied the spatial position of these L-shaped patterns, i.e. they were placed either in the middle or in one of the corners of a small work field ('drawing box'). Subjects were asked to copy the pattern in a similar position. To answer the second question concerning inhibition, we used an extra task in which the point from which the subject had to start copying the L-shaped patterns was imposed (indicated by a black dot in the pattern itself). This compelled the subjects to either start copying at the generally most preferred starting point or to start at a less preferred starting point.

In order to avoid a 'trade-off' effect between initiation time and pen-up time, we did not use patterns consisting of three or more line segments. It was assumed that in copying one-segment or two-segment patterns the initiation period would adequately reflect the time needed for visualspatial processing and motor planning.

Based on the hypothesis that schizophrenia is associated with a rather basic or generalized psychomotor slowing (King, 1991; Goldberg \& Gold, 1995; Schatz, 1998; Jogems-Kosterman et al., 2001), we anticipated that, compared to controls, the patients would even show longer initiation times when copying single lines. In contrast, we did not expect patients to be disproportionately slower in copying two-segment patterns. Because the conflict between the graphic rules present in the two-segment patterns was likely to be resolved before participants started copying, we predicted that the effects of this conflict would be mainly reflected in the initiation time. Moreover, in line with our previous findings (Jogems-Kosterman et al., 1999 and Chapter 5; Hulstijn et al, 2001) we anticipated that this effect would be larger for the patients than for the controls.

It has been reported earlier that patients with schizophrenia show deficits on visual-spatial working memory tests (Pantelis et al., 1997; Keefe et al., 1997; Fleming et al., 1997). On the other hand, their basic visual-spatial ability appears to be intact (Keefe et al., 1997; Fleming et al., 1997). Fleming et al. (1997), for example, found that patients with schizophrenia were able to copy a dot in 
the same location as they saw it on the stimulus page. In general, we proposed that drawing a pattern in one of the corners of a designated field makes a greater appeal on movement planning because of the additional spatial demand in the task. More than when drawing the pattern in the middle of the space, subjects, now, have to take into consideration the relative distance between their drawing and the borders of the box. It was therefore expected that the position of the pattern would affect the initiation time. We also hypothesized that the patients would show longer initiation times than the controls when the figure had to be drawn in one of the corners of the box.

Previous studies have shown that patients with schizophrenia have difficulties with the inhibition of a strongly cued automatic response (e.g., Marczewski, Van der Linden \& Larøi, 2001; Barch \& Carter, 1998; Everett, Laplante \& Thomas, 1989). Our extra task, in which the starting point was imposed, does not actually demand the inhibition of a particular response but rather the inhibition of applying the most obvious graphic production rule. We hypothesized that forcing subjects to inhibit the application of a highly preferred rule (particularly starting at the left and/or at the top) and begin their drawing at a less preferred position would increase the initiation time. Moreover, we anticipated that this effect would be larger in the schizophrenia group.

\section{Method}

\section{Participants}

Twenty patients (16 male; 4 female; mean age (SD) $=41.8(9.5)$; median educational level was high school) who met the criteria for schizophrenia (DSM-III-R, American Psychiatric Association, 1987) participated in the study. The patients were all receiving long-term care within our institute, the GGZ Oost Brabant. Seven patients were using a classical antipsychotic drug, nine patients were taking clozapine and two patients were treated with an atypical antipsychotic drug. Two other patients were using a combination of these drugs (a classical antipsychotic drug plus clozapine or an atypical antipsychotic drug). Besides this antipsychotic medication, some patients also received an antidepressant agent $(n=6)$, a benzodiazepine $(n=13)$, an antiparkinson agent $(n=8)$ or an anticonvulsant $(\mathrm{n}=2)$

The control group (14 male; 6 female) consisted of employees of the same institute matched for age (mean age $(\mathrm{SD})=41.6(9.6)$ ) and education (median educational level was 'high school'). In both groups, 18 subjects were right-handed and two were left-handed.

\section{Clinical assessment}

For the schizophrenia group psychopathology was assessed using the Positive and Negative Syndrome Scale (PANSS) (Kay, Fiszbein \& Opler, 1987). The mean scores on the positive symptoms, negative symptoms and general psychopathology subscale were 15.0 (S.D. = 5.8), 13.3 $($ S.D. $=6.1), 31.0($ S.D. $=6.5)$, respectively. To determine the correlation between the PANSS scores and task performance a subdivision was made between positive symptoms, negative symptoms and disorganization symptoms (see also Cameron et al., 2002). The mean sum score for 


\section{2 | Chapter 7}

the positive cluster symptoms was 12.8 (sum of 5 items), for the negative cluster symptoms 13.2 (sum of 7 items) and for the disorganization cluster symptoms 10.2 (sum of 4 items).

\section{Apparatus, tasks and procedure}

In the present study subjects performed a number of copying tasks. The stimuli to be copied were displayed on a computer screen. The participants' performance on these tasks was recorded by means of a digitizing tablet (WACOM 1218RE). The copying took place on normal sheets of paper that were put on the tablet. Squares $(30$ by $30 \mathrm{~mm}$ ) on the sheets indicated the location where the patterns had to be copied. To start each trial the participants had to place the pen in a circle that was positioned at the lower left corner of the square. After a random waiting period (ranging between 500 and $2000 \mathrm{~ms})$ the stimulus appeared on the screen accompanied by a warning signal $(1000 \mathrm{~Hz}$, $300 \mathrm{~ms}$ ). Participants were instructed to copy the stimulus as fast and as accurately as possible. As soon as the subject started drawing the stimulus disappeared from the screen. Although they were told that emphasis was on speed rather than neatness, the participants were asked to keep an eye on the proportions of the line segments. After completing the drawing, the pen had to be placed and held stationary in a circle at the upper right corner of the square for at least $300 \mathrm{~ms}$ (followed by a low tone of $500 \mathrm{~Hz}$ ).

\section{One-segment stimuli:}

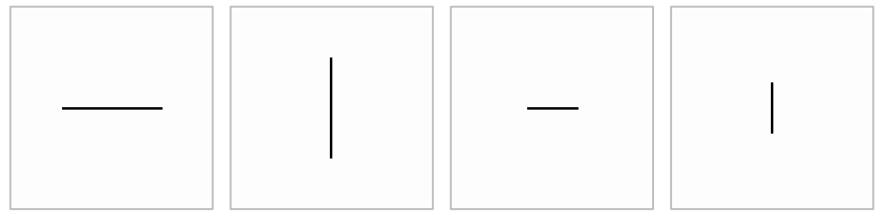

Two-segment stimuli (non-conflicting):
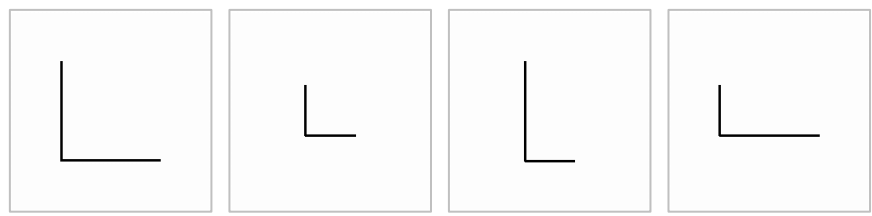

Two-segment stimuli (conflicting):

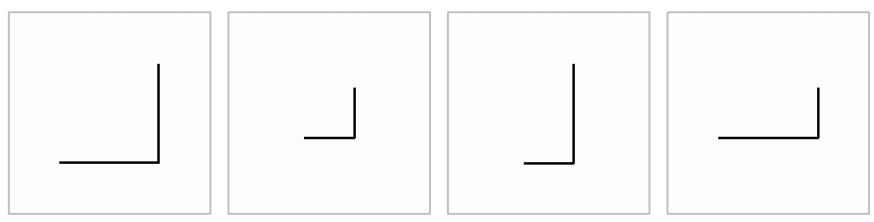

Figure 1: Stimuli used in the first copying task. 


\section{Copying patterns consisting of one and two segments}

In this task, participants were asked to copy one- and two-segment stimuli (see Figure 1). The twosegment stimuli consisted of either L-shaped patterns that could be copied easily according to the graphic production rules (non-conflicting patterns), or patterns in which subjects had to violate one or more of these rules (conflicting patterns). To avoid response tendencies, we varied the length of the line-segments. All stimuli were copied six times in a random order.

\section{Copying L-shaped patterns with an additional spatial component}

In this task the spatial position of the presented patterns was varied (see Figure 2). The L-shaped patterns were placed either in the middle of the drawing box, or in one of its four corners. A differentiation was also made between non-conflicting (NC) and conflicting (C) patterns. Participants were asked to copy the patterns in the same position in which they were presented on the computer screen. All ten patterns were copied four times in a random order.
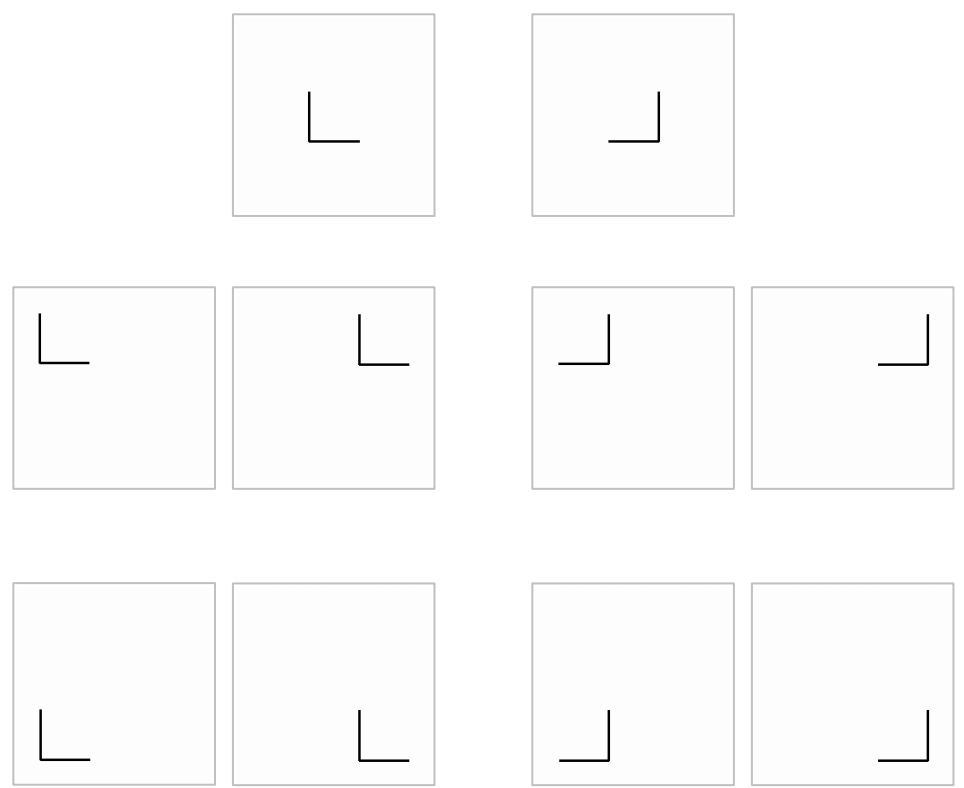

Figure 2: L-shaped patterns with an additional spatial component. The two topmost images show the 'key' patterns placed in the middle of the indicated box. The other examples depict the same stimulus patterns, but now positioned in each of the four corners of the box. The five left-hand patterns are non-conflicting (NC), whereas the five right-hand patterns are conflicting $(\mathrm{C})$.

\section{Copying L-shaped patterns with an imposed starting point}

The L-shaped patterns used in this task resembled those of the first task. However, here participants were instructed to start drawing the pattern at the position indicated by a black dot that was indicated in the stimulus pattern itself (see Figure 3). This implied that the imposed starting point either corresponded to or deviated from the normal preference. This preference could be either 
strong (original $\mathrm{NC}$ patterns) or weaker (original C patterns). Each pattern was presented four times in a random order.

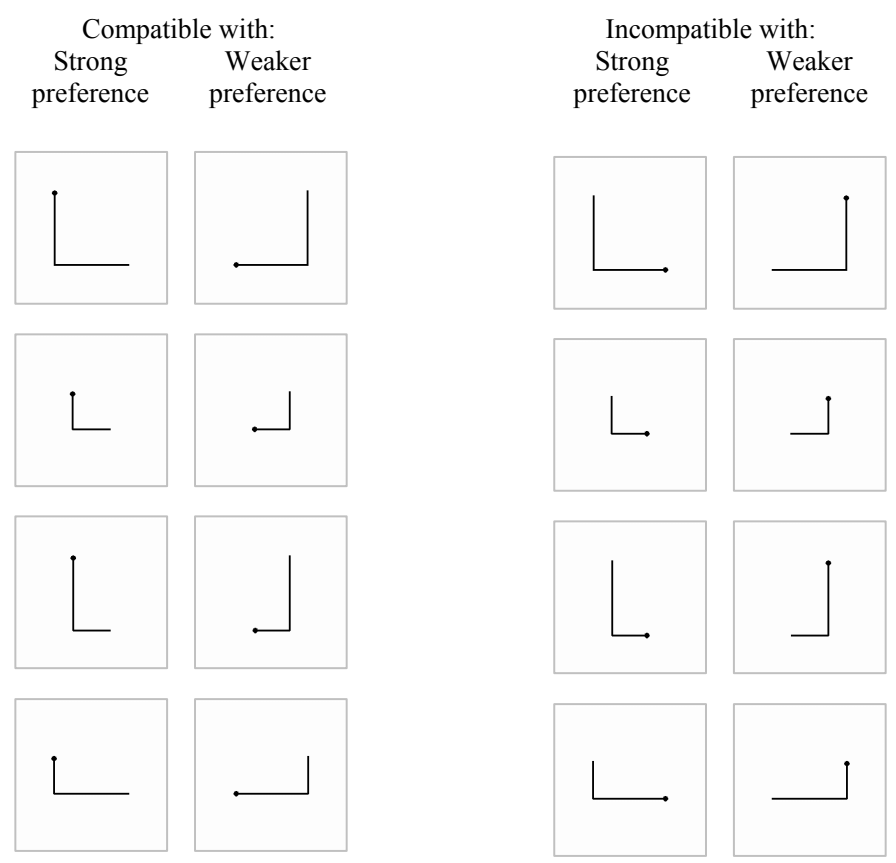

Figure 3: The stimulus patterns with a starting point that either supported or opposed a normal preference, which preference could either be strong or weaker.

\section{Recording and analysis}

During drawing, the $\mathrm{x}$ and $\mathrm{y}$ coordinates of the pen on, and $5 \mathrm{~mm}$ above, the digitizer were recorded with a frequency of $200 \mathrm{~Hz}$ and a spatial accuracy of $0.2 \mathrm{~mm}$. Based on pen pressure (threshold was assessed for each trial separately, but ranged around $0.24 \mathrm{~N}$ ), movement trajectories were defined as either pen-up periods or pen-down periods.

The main dependent variable was initiation time, being the period between the appearance of the stimulus on the computer screen and the start of the first pen-down movement. In addition, we analyzed the execution period, divided into movement time, calculated as the accumulated pendown periods, and (if applicable) pen-up time, the latter calculated as the accumulated pen-up periods.

Data analyses were performed using SPSS for Windows. For each of the movement variables, statistical evaluation was performed with analysis of variance (GLM for repeated measures) with Group as a between-subject factor. In the task in which one- and two-segment patterns were copied we used the following within-subject factors: 'number of lines' and 'NC versus $C^{\prime}$. In the task in which patterns were copied with an additional spatial component, the within-subjects factors were the 'spatial position' (center versus corner) and 'NC versus C'. In the task in which the starting point was imposed the following within-subject factor was used: 
'compatible with versus incompatible with normal preference'. In this latter task we did not compare $\mathrm{NC}$ and $\mathrm{C}$ patterns, because now the extra instruction to start drawing at a particular point could in fact change a $\mathrm{NC}$ pattern into a $\mathrm{C}$ pattern.

For the task in which participants copied L-shaped patterns with a forced starting point, we also examined the percentage of errors (i.e., when a subject did not start drawing at the black dot) and tested these by means of a Mann-Whitney U Test. For the patient group, correlations between clinical rating scores and initiation times were calculated using Pearson's correlation coefficient. Differences in task performance between types of antipsychotic medication (classical antipsychotic drug versus clozapine / atypical antipsychotic drug) were examined by means of a $t$-test.

Our study also included a number of left-handed persons. Theoretically (see Thomassen \& Tibosch, 1991), one could expect differences in the application of graphic production rules and, as a consequence, also in the amount of conflicts experienced between right-handed and left-handed persons. For example, whereas right-handed individuals will prefer drawing lines from left-to-right, left-handers might favor drawing in the opposite direction. However, in our study groups there were no clear indications that the four left-handed persons formed a separate cluster of subjects. Therefore, we decided to analyze the results of the groups as a whole.

\section{Results}

Copying one- and two-segment patterns

The results of the task with the one-segment stimuli showed that the schizophrenia patients were not significantly slower in their initiation time $(F(1,36)=2.08, p=0.16)$ and movement time $(F(1,36)=$ $0.73, \mathrm{p}=0.40$ ) when compared to the controls. For the means of initiation time see Figure 4. Although copying the two-segment stimuli did lead to a significant prolongation of initiation time $(\mathrm{F}(1,35)=21.18, \mathrm{p}<0.001)$, this prolongation proved not to be significantly different for the two groups (group X number of lines: $F(1,35)=2.15, \mathrm{p}=0.15$; see Figure 4).

The two-segment stimuli used in this task consisted of patterns that could either be easily copied according to the graphic production rules (NC patterns) or patterns that would lead to conflicts between some of the rules (C patterns) while copying. Indeed, it appeared that the overall effect of $\mathrm{NC}$ versus $\mathrm{C}$ on initiation time was significant $(\mathrm{F}(1,35)=4.96, \mathrm{p}<0.05)$. However, this effect was similar for both groups: the interaction between $\mathrm{NC}$ versus $\mathrm{C}$ and group was not significant $(\mathrm{F}(1,35)=0.001, \mathrm{p}=0.98)$. Overall, there was no group effect for initiation time $(\mathrm{F}(1,35)=0.66, \mathrm{p}=0.42)$.

Also movement time was increased in the C condition (1078 ms) when compared to the $\mathrm{NC}$ condition $(1044 \mathrm{~ms})(\mathrm{F}(1,35)=5.31, \mathrm{p}<0.05)$, but again, this effect was about equal for both patients and controls; there was no significant group by $\mathrm{NC}$ versus $\mathrm{C}$ interaction $(\mathrm{F}(1,35)=1.38, \mathrm{p}$ $=0.25)$. Patients did not show significantly longer movement times $(1068 \mathrm{~ms})$ when compared to the controls $(1053 \mathrm{~ms})(\mathrm{F}(1,35)=0.02, \mathrm{p}=0.90)$. Overall, the mean duration of pen-up periods was almost negligible (about 50\% of the subjects did not lift their pen in any of the drawings). Pen-up periods, however, were longer in the $\mathrm{C}$ condition when compared to the $\mathrm{NC}$ condition $(\mathrm{F}(1,35)=$ 
4.63, $\mathrm{p}<0.05)$. There was no significant difference in the mean pen-up duration between patients and controls $(\mathrm{F}(1,35)=0.002, \mathrm{p}=0.96)$.

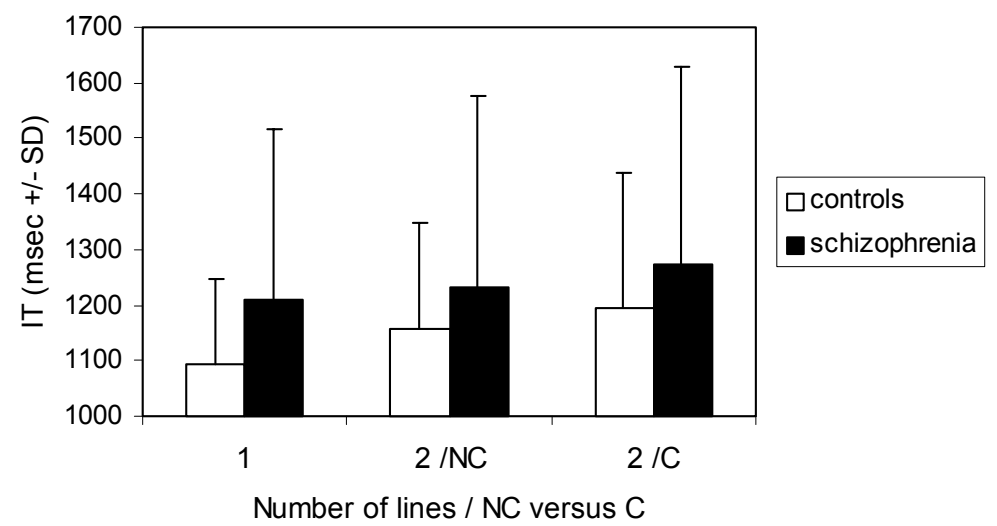

Figure 4: Mean values and standard deviations of initiation time (IT) as a function of number of lines. The two-segment patterns are divided into non-conflicting patterns (NC) and conflicting patterns (C).

\section{Copying L-shaped patterns with an additional spatial component}

In the analysis of this task we contrasted the two 'key' patterns that were placed in the middle of the designated drawing area ('center') with the eight patterns that were positioned in one of four corners of the box ('corner'). We also compared the NC patterns with the C patterns. The mean values of initiation time are displayed in Figure 5.

The position of the pattern (either in the middle or in a corner) appeared to have a highly significant effect on initiation time $(\mathrm{F}(1,36)=46.52, \mathrm{p}<0.001)$. Moreover, this position effect was greater in the schizophrenia group than in the control group: the interaction between group and condition (center, corner) was significant $(\mathrm{F}(1,36)=5.81, \mathrm{p}<0.05)$. Overall, schizophrenic patients were also significantly slower in their initiation times when compared to the control subjects $(\mathrm{F}(1,36)=8.73, \mathrm{p}<0.01)$. In this task, no difference was found between the NC and $\mathrm{C}$ patterns $(\mathrm{F}(1,36)=0.64, \mathrm{p}=0.43)$, and, although the initiation times for the eight 'corner' stimuli was found to be different $(F(7,30)=5.75, p<0.001)$, this effect was not significantly different for the two groups (group $\mathrm{X}$ corner stimuli: $\mathrm{F}(7,30)=1.71, \mathrm{p}=0.14$ ). The eight 'corner' stimuli do not only differ in relative position with respect to the middle pattern, but also in the space that is left or has to be planned for the second segment. For example, the L-type patterns can be more easily planned or drawn when they are positioned in a left-hand corner than when they are placed near the right periphery of the drawing box. The reversed L's are more difficult in a left-hand position. When we analyzed this factor, i.e., available space for the second segment, separately from corner position (averaged over $\mathrm{NC}$ and $\mathrm{C}$ ), we found no significant effect of available space on initiation time $((\mathrm{F}(1,34)=0.021, \mathrm{p}=0.89)$ and no significant interactions with group $(\mathrm{F}(1,34=0.09, \mathrm{p}=0.76)$. 
Also movement time was increased when patterns had to be drawn in one of the corners $(911 \mathrm{~ms})$ instead of in the center $(833 \mathrm{~ms})(\mathrm{F}(1,36)=18.34, \mathrm{p}<0.001)$. This effect was again similar for patients and controls $(\mathrm{F}(1,36)=0.26, \mathrm{p}=0.61)$. It was apparent that copying $\mathrm{C}$ patterns resulted in significantly longer movements when compared to the NC patterns $(\mathrm{F}(1,36)=11.58, \mathrm{p}<$ 0.01 ). In addition, this difference in movement duration between $\mathrm{NC}$ and $\mathrm{C}$ was significantly greater in the schizophrenia group than in the control group $(\mathrm{F}(1,36)=5.36, \mathrm{p}<0.05)$. Movement time differed significantly between the eight 'corner' stimuli $(F(7,30)=4.97), p<0.001$. This effect, however, was not significantly different between patients and controls (group X corner stimuli: $F(7,30)=0.86, p=0.55)$. With respect to the corner positions it was found that the patterns in which the second segment had to be drawn close to the border of the box were copied with significantly longer movement times $(\mathrm{F}(1,36)=9.98, \mathrm{p}<0.01$.

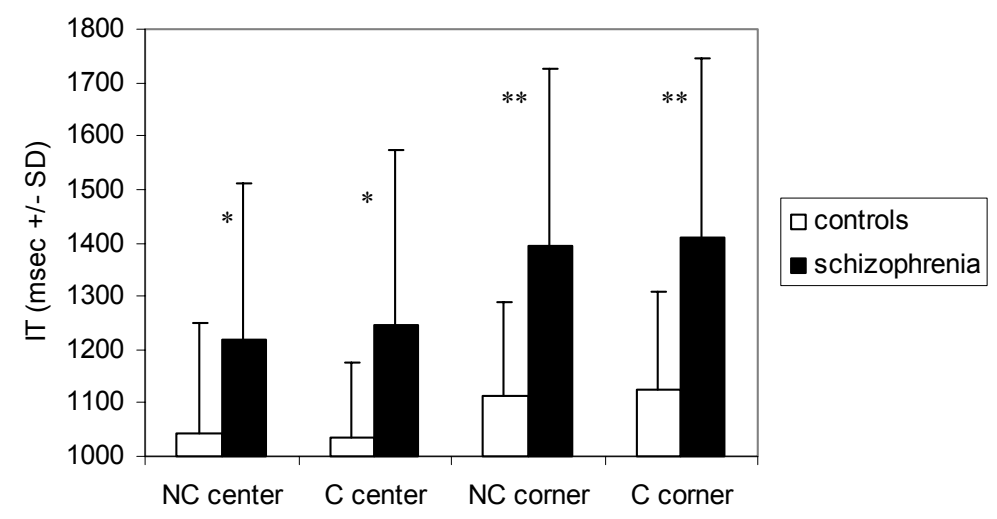

Figure 5: Mean values and standard deviations of initiation time (IT) in copying patterns that were either placed in the center of a box (no extra spatial component) or in one of the four corners of the box (imposing an additional spatial component). A differentiation is made between non-conflicting (NC) and conflicting (C) patterns. ** $\mathrm{P}<0.01, * \mathrm{P}<0.05$

\section{Copying L-shaped patterns with an imposed starting point}

In some of the patterns in this task, the compulsory starting point corresponded to the most preferred starting point (patterns shown in the two left-hand columns of Figure 3). For example, while copying the same L-shaped figure (with equal segment lengths) in the control condition all subjects started drawing at the top left. In copying the mirror image of this figure, the most preferred starting point, although less frequently (controls: 74\%, schizophrenic patients: 63\%), was bottom left. The patterns shown at the right-hand side of Figure 3, on the other hand, challenged most subjects to initiate their drawing at a less preferred point, namely the top right or bottom right.

The mean values of initiation time are displayed in Figure 6. These means were calculated on the drawings that were produced correctly. Occasionally, participants did not start drawing as instructed by the black dot. On average, the patients made slightly more errors $(2.0 \%)$ when 
compared to the controls $(0.5 \%)$. This difference, however, was not significant $(\mathrm{U}=148.5, \mathrm{p}=$ $0.08)$.

Initiation time was significantly longer in the schizophrenia patients than in the control subjects $(\mathrm{F}(1,38)=7.80, \mathrm{p}<0.01)$. Furthermore, forcing the participants to start drawing at a point that opposed their normal preference (either strong or weaker) led to a significantly larger initiation time $(\mathrm{F}(1,38)=24.90, \mathrm{p}<0.001)$. The most important result, however, was that the initiation time of patients was more affected by this difference between the compatible and the incompatible condition than that of the controls $(\mathrm{F}(1,38)=6.32, \mathrm{p}<0.05)$. Post-hoc analyses (using repeated contrasts) showed that, averaged over all subjects, the first condition (i.e., compatible with a strong preference) and the second condition (i.e., compatible with a weaker preference) did not significantly differ in IT $(\mathrm{F} 1,38)=0.10, \mathrm{p}=0.75$. However, the interaction between these two conditions and group was found to be significant $(\mathrm{F}(1,38)=5.35, \mathrm{p}<0.05)$. It appeared that the patients were relatively faster in the second condition than in the first condition when compared to the controls. The third and the fourth condition did not differ from each other in IT and there was no interaction with group.

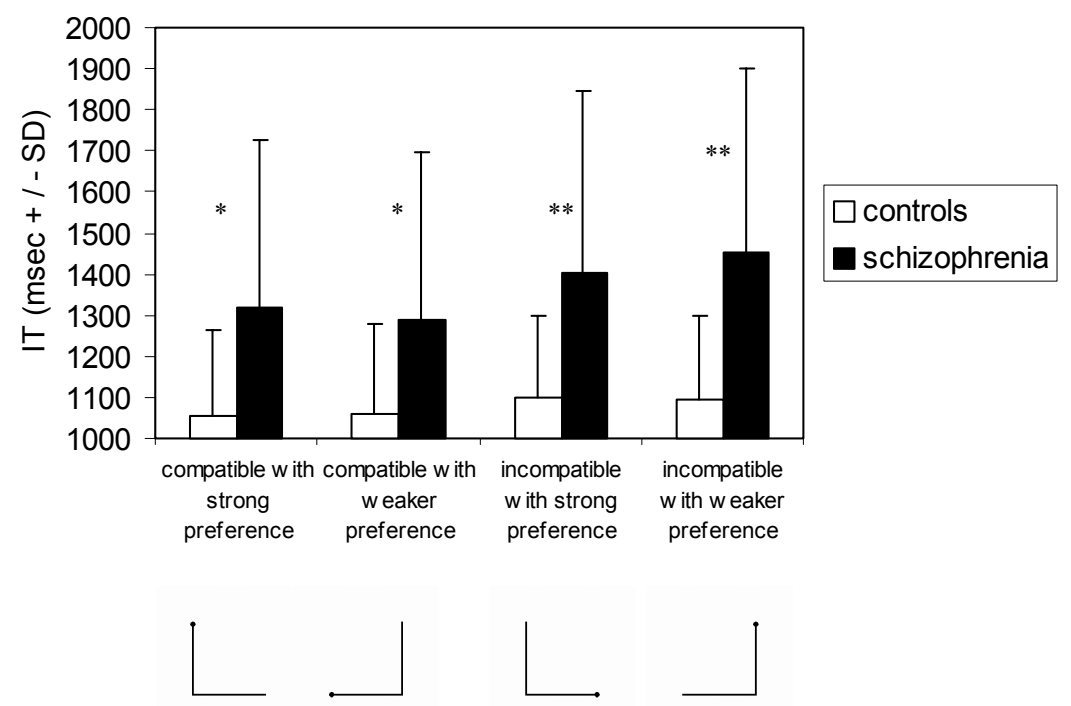

Figure 6: Mean values and standard deviations of initiation time (IT) in copying patterns with an imposed starting point that either corresponded or opposed a normal preference, which preference could additionally be either strong or weaker. ${ }^{* *} \mathrm{P}<0.01,{ }^{*} \mathrm{P}<0.05$

Movement time was also significantly increased when participants were challenged to start drawing at a point that opposed their normal preference $(1104 \mathrm{~ms})$ when compared to the condition in which the imposed starting point corresponded with this preference $(1084 \mathrm{~ms})(\mathrm{F}(1,38)=5.14), \mathrm{p}$ $<0.05$ ). This effect, however, was not greater for the patients than for the controls (group $\mathrm{X}$ 'compatible with versus incompatible with normal preference': $F(1,38)=0.78, p=0.38$ ). 
Movement time was not prolonged in the patients compared to that of the controls $(\mathrm{F}(1,38)=1.49$, $\mathrm{p}=0.23)$.

Pen-up periods, again, did not occur very often (more than $60 \%$ of the subjects drew all the patterns without lifting the pen). The mean pen-up duration was not affected by the difference between the preferred and non-preferred starting points $(\mathrm{F}(1,38)=2.19, \mathrm{p}=0.15)$. Overall, patients and controls showed similar mean pen-up times $(F(1,38)=0.002, p=0.97)$.

\section{Relationship with clinical measures}

The correlation coefficients between initiation time and scores for positive, negative and disorganized symptoms are shown in Table 1. We computed an average initiation time over the NC and $\mathrm{C}$ conditions. Positive symptoms did not correlate with any of the initiation times, although the correlation coefficient tended to be slightly higher when L-shaped patterns with an additional spatial component were being copied. Negative symptoms were not significantly correlated with the duration of the initiation periods. However, the correlation coefficient tended to be higher in the imposed task condition. Disorganization symptoms appeared to be significantly correlated with the duration of the initiation period when patterns with a preferred starting point were copied: remarkably, higher disorganization scores were related to shorter initiation times. The other, also negative, correlation coefficient in the forced task condition (i.e., the non-preferred condition) approached significance.

To verify whether task performance was related to the type of antipsychotic medication participants were taking, we conducted a $t$-test, which revealed that initiation time did not differ between patients using classical antipsychotic medication and patients receiving atypical antipsychotic agents (including clozapine).

Table 1: Correlations between initiation time and clinical rating scores.

\begin{tabular}{llll}
\hline Initiation time & Positive symptoms & Negative symptoms & Disorganized symptoms \\
\hline & & & \\
One-line pattern & -0.08 & 0.31 & -0.05 \\
Two-segment patterns & -0.05 & 0.28 & -0.05 \\
Spatial condition: & & & \\
$\quad$ Center & 0.29 & 0.16 & -0.24 \\
$\quad$ Corner & 0.29 & 0.15 & -0.32 \\
$\quad$ & & $-0.47^{*}$ \\
$\quad$ Imposed starting condition: & -0.08 & $0.45^{\#}$ & $-0.39^{\#}$ \\
$\quad$ Preferred & -0.01 & 0.35 &
\end{tabular}

\section{Discussion}

The results of the present study demonstrated that the patients with schizophrenia were not slower in the initiation and execution of either the one- or the two-line drawing movements. However, 
when the visual-spatial requirements of the task were enhanced, patients needed more time for planning: compared to the controls they showed significantly longer initiation times when a pattern had to be drawn in a corner, instead of in the center of the drawing box. Further, when they had to inhibit a highly preferred response pattern (i.e., were forced to start copying at a less preferred starting point), patients needed significantly more initiation time than the controls.

The finding that the patients with schizophrenia did not show prolonged initiation times while copying simple one-line patterns seems to contrast with the findings of our previous study (Jogems-Kosterman et al., 2001, see Chapter 4) in which we found that patients with schizophrenia needed longer initiation times when copying one line. However, in this earlier study we did not only use horizontal or vertical lines (like in the present study), but also diagonal lines. One may assume that in copying diagonal lines both visual-spatial and motor demands are greater than in copying vertical or horizontal lines. This may also account for the results of two previous studies on movement planning (Malla et al., 1995; Carnahan et al., 1997), in which it was found that patients with schizophrenia were retarded in initiating manual movements towards targets. The planning of goal-directed aiming movements is presumably more difficult than freely copying lines, as it requires a more precise planning of the end-state of the movement. In conclusion, our first assumption that patients with schizophrenia would show slowing even when copying one single line was not confirmed. Obviously, schizophrenia is not necessarily associated with motor slowing. Our previous investigations with the Digit Symbol Test (DST; Van Hoof et al., 1998 and Chapter 2; Jogems-Kosterman et al., 2001 and Chapter 4) indeed revealed that patients with schizophrenia were not retarded in their movements, i.e., did not show a prolongation in the time they needed to write the digits, although they did need more time for finding the right digit (so-called matching time).

Our second prediction that patients with schizophrenia would not be disproportionately slower in copying two segment-patterns was confirmed: the patients with schizophrenia were not significantly slower in their initiation time while copying L-shaped patterns. Contrary to our expectations, the patients did not show prolonged initiation times when copying 'conflicting' Lshaped patterns (in which conflicts occurred between graphic production rules) when compared to the controls. However, in our previous study (Hulstijn et al., 2001), in which we used both two- and three-segment patterns, we also found a less substantial effect of conflicting rules on the initiation period. Particularly in the three-segment patterns, patients with schizophrenia displayed deviant pen-up periods during the drawing phase (Hulstijn et al., 2001). Thus, it seems that augmenting the number of line-elements may be crucial to provoke planning difficulties in patients with schizophrenia. Yet, the consequence of this increase in the number of lines may be that the capacity of the participants' working memory will play a more important role. Overall, the present findings suggest that the performance on the simple two-segment patterns may not be optimal indicators of planning deficits in schizophrenia.

In line with our hypothesis, copying patterns in a corner, instead of in the center of a drawing box appeared to significantly influence initiation time. Moreover, as predicted, this effect was greater in the schizophrenia group. One reason for this could be that the spatial constraints when having to copy a pattern in a corner are larger than when patterns need to be drawn in the 
middle of the box. Possibly, patients with schizophrenia encounter more problems in visual-spatial planning. A related explanation is that the patients experienced difficulties with the planning of those patterns that left them relatively less room for the second segment. However, the effect of this planning constraint on initiation time was not significantly different for the two groups. Thus, an alternative explanation for the findings is that the more normal position for copying a pattern within a square is in the middle of that square. To realize variations of this position, the general tendency to draw in the middle of the box needs to be suppressed. Patients with schizophrenia may be less able to inhibit well-learned tendencies.

As predicted, forcing subjects to start copying at a less preferable starting point appeared to significantly prolong initiation time. Also in line with our expectation was that this effect was greater in the schizophrenia group. The patients tended to make more errors (i.e., they did not always start drawing at the black dot). In general, these findings correspond with studies showing that schizophrenia is associated with impaired cognitive inhibition (e.g., Marczewsky, Van der Linden \& Larøi, 2001; Barch \& Carter, 1998; Everett, Laplante \& Thomas, 1989). Our results suggest that patients encountered more problems when they could not start drawing at a preferred starting point. In the non-preferred starting condition, participants have to inhibit a more or less automatic response. On the other hand, patients did not take advantage of the condition in which the indicated starting point corresponded with a preferred movement onset. Also in the preferred condition patients were significantly slower than controls. Perhaps the additional instruction to start at a particular point in itself may have caused a certain slowing of the initiation time.

How do these results relate to clinical measures of schizophrenia? First, it was apparent that in the patient group performance on the copying tasks did not correlate significantly with negative symptoms. There was only a tendency for the association to become stronger in the more complex compulsory task condition: higher negative symptom scores involved longer initiation times in copying L-shaped patterns with an imposed starting point. In general, this result corresponds with the findings of our previous study (Jogems-Kosterman et al., 2001, see Chapter 4), in which the severity of negative symptoms was related to initiation time only in the most complex task condition. Positive symptoms were not related to the length of the initiation times. This finding is in line with several other studies (e.g., Basso et al., 1998; Brazo et al., 2002) showing almost intact neuropsychological functioning in patients with predominantly positive symptoms. Symptoms of disorganization were associated with shorter initiation times in the forced task condition. Apparently, patients showing clinical features of cognitive disorganization react faster than other patients when they are asked to start drawing at an indicated point. The explanation of this result may be that patients with disorganization tend to display so-called stimulus-driven behavior more strongly (Frith, 1992). In our forced task condition, the starting dot may have served as a stimulus allowing the reaction time to be shortened, irrespective of whether this stimulus corresponded with a preferred starting point or not.

In conclusion, patients with schizophrenia may show fairly intact initiation of simple movements. On the other hand, difficulties with inhibiting an automatic response tendency presumably play a crucial role in the planning problems of these patients. In general, our results fit in surprisingly well with a theory that has recently been proposed to elucidate the pathogenesis of 
schizophrenia (Van Hoof, 2002). Following this theory two brain mechanisms are of relevance: a dorsal "guidance" mechanism for response inhibition and the planning of actions, and a ventral "drive" mechanism for the initiation of actions. Patients who are mildly disabled (with a failure of the dorsal mechanism; patients with predominantly psychotic symptoms and/or cognitive disorganization) should have no initiation problems or even show overcompensation. In severe psychopathology (with a failure in both mechanisms; patients with mainly negative symptoms), an additional general slowing can be expected. The notion that basic cognitive malfunctioning is a crucial aspect of schizophrenia (Andreasen, 1999) has recently been gaining ground. Certain cognitive functions may also have predictive value for the functional outcome of patients with schizophrenia (Green, 1996; Green et al., 2000). Particularly impaired executive functioning which following Lezak (1995) includes volitional activity, forward planning, cognitive flexibility and self-regulation - has been found to be related to poor future outcome. Wykes et al. (Wykes, et al., 1992; Wykes, 1994; Wykes, Reeder \& Corner, 2000), for example, found that a complex reaction time measure, which was thought to assess response inhibition, was predictive of future dependence on psychiatric services. Following this line of research, we feel that the tasks used in the present study may be useful to understand cognitive deficits and problems in the everyday functioning of patients with schizophrenia in the perspective of the future outcome for these patients. This is also the subject of our most recent study (Fakkers et al., 2002). 
Movement planning deficits in schizophrenia: Failure to inhibit automatic response tendencies | 123

\section{References}

American Psychiatric Association (1987). Diagnostic and statistical manual of mental disorders (3th edition revised).Washington, D.C.: APA.

Andreasen, N. (1999). A unitary model of schizophrenia. Bleuler's "fragmented phrene" as schizencephaly. Archives of General Psychiatry, 56, 781-787.

Barch, D.M., \& Carter, C.S. (1998). Selective attention in schizophrenia: Relationship to verbal working memory. Schizophrenia Research, 33, 53-61.

Basso, M.R., Nasrallah, H.A., Olson, S.C., \& Bornstein, R.A. (1998). Neuropsychological correlates of negative, disorganized and psychotic symptoms in schizophrenia. Schizophrenia Research, 31, 99-111.

Brazo, P., Marié, R.M., Halbecq, I., Benali, K., Segard, L., Delamillieure, P., Langlois-Théry, S., Van Der Elst, A., Thibaut, F., Petit, M., \& Dollfus, S. (2002). Cognitive patterns in subtypes of schizophrenia. European Psychiatry, 17, 155-162.

Cameron, A.M., Oram, J., Geffen, G.M., Kavanagh, D.J., McGrath, J.J., \& Geffen, L.B. (2002) Working memory correlates of three symptom clusters in schizophrenia. Psychiatry Research, 110, 49-61.

Carnahan, H., Aguilar, O., Malla, A., \& Norman, R. (1997). An investigation into movement planning and execution deficits in individuals with schizophrenia. Schizophrenia Research, 23, 213-221.

Clare, L., McKenna, P.J., Mortimer, A.M., \& Baddeley, A.D. (1993). Memory in schizophrenia: What is impaired and what is preserved? Neuropsychologia, 31, 1225-1241.

Duncan, J. (1995). Attention, intelligence, and the frontal lobes. In: M.S. Gazzaniga (Ed.), The cognitive neurosciences (pp. 721-733). Cambridge: MIT Press.

Everett. J., Laplante, L., \& Thomas, J. (1989). The selective attention deficit in schizophrenia: Limited resources or cognitive fatigue? Journal of Nervous and Mental Disease, 177, 735-738.

Fakkers, G.P.F.M., Jogems-Kosterman, B.J.M., Loonen, A.J.M., Van Hoof, J.J.M., \& Hulstijn, W. (2002). Changes in clinical functioning and neurocognitive performances in patients with schizophrenia during the first years of deinstitutionalization. Schizophrenia Research, 53, 127.

Fleming, K., Goldberg, T.E., Binks, S., Randolph, C., Gold, J.M., \& Weinberger, D.R. (1997). Visuospatial working memory in patients with schizophrenia. Society of Biological Psychiatry, 41, 43-49.

Frith, C.D. (1992). The cognitive neuropsychology of schizophrenia. Hove: Lawrence Erlbaum Associates.

Goldberg, T.E., Torrey, E.F., Gold, J.M., Ragland, J.D., Bigelow, L.B., \& Weinberger, D.R. (1993). Learning and memory in monozygotic twins discordant for schizophrenia. Psychological Medicine, 23, 71-85.

Goldberg, T.E., \& Gold, J.M. (1995). Neurocognitive deficits in schizophrenia. In: S.R. Hirsch \& D.R. Weinberger (Eds.). Schizophrenia (pp. 146-162). Oxford: Blackwell Science.

Green, M.F. (1996). What are the functional consequences of neurocognitive deficits in schizophrenia? American Journal of Psychiatry, 153, 321-330.

Green, M.F., Kern, R.S., Braff, D., \& Mintz, J. (2000). Neurocognition and functional outcome in schizophrenia: Are we measuring the right stuff? Schizophrenia Bulletin, 26, 119-136.

Hanes, K.R., Pantelis, C., Andrewes, D., \& Chiu, E. (1996). Subcortical dysfunction in schizophrenia: A comparison with Parkinson's disease and Huntington's disease. Schizophrenia Research, 19, 121-128. 
Hulstijn, W. (1994). Figure copying and retardation in depression. In: C. Faure, P. Keuss, G. Lorette \& A. Vinter (Eds.). Advances in handwriting and drawing: A multidisciplinary approach (pp. 447-488). Paris: Europia.

Hulstijn, W., Jogems-Kosterman, B.J.M., Wezenberg, E., \& Sabbe, B.G.C. (2001). An evaluation of the use of figure-copying tasks in studies of planning deficits in schizophrenia. In: R.G.J. Meulenbroek \& B. Steenbergen (Eds.), Proceedings of the tenth biennial conference of the International Graphonomics Society (pp. 46-51). Nijmegen: IGS.

Jogems-Kosterman, B.J.M., Hulstijn, W., Van Hoof, J.J.M., \& Thomassen, A.J.W.M. (1999). Is the planning of action more impaired in schizophrenic patients than in depressed patients? The effects of conflicting graphic production rules. In: G. Leedham, M. Leung, V. Sagar \& X. Xuhong (Eds.), Proceedings of the 9th biennial conference of the International Graphonomics Society (pp. 225-229). Singapore: NTU.

Jogems-Kosterman, B.J.M., Zitman, F.G., Van Hoof, J.J.M., \& Hulstijn, W. (2001). Psychomotor slowing and planning deficits in schizophrenia. Schizophrenia Research, 48, 317-333.

Kay, S.R., Fizsbein, A., \& Opler, L.E. (1987). The Positive and Negative Syndrome Scale (PANSS) for schizophrenia. Schizophrenia Bulletin, 13, 261-275.

Keefe, R.S.E., Lees-Roitman, S.E., \& Dupre, R.L. (1997). Performance of patients with schizophrenia on a pen and paper visuospatial working memory task with short delay. Schizophrenia Research, 26, 9-14.

King, H.E. (1991). Psychomotor dysfunction in schizophrenia. In: S.R. Steinhauer, J.H. Gruzelier \& J. Zubin

(Eds.), Handbook of schizophrenia, Vol. 5: Neuropsychology, psychophysiology and information processing (pp. 273-301). Amsterdam: Elsevier Science Publishers.

Krabbendam, L., De Vught, M.E., Derix, M.M.A., \& Jolles, J. (1999). The Behavioural Assessment of the Dysexecutive Syndrome as a tool to assess executive functions in schizophrenia. The Clinical Neuropsychologist, 13, 370-375.

Lezak, M.D. (1995). Neuropsychological assessment (3rd edition). Oxford: University Press.

Malla, A.K., Norman, R.M.G., Aguilar, O., Carnahan, H., \& Cortese, L. (1995). Relationship between movement planning and psychopathology profiles in schizophrenia. British Journal of Psychiatry, 167, 211-215.

Marczewski, P., Van der Linden, M., \& Larøi, F. (2001). Further investigation of the Supervisory Attentional System in schizophrenia: Planning, inhibition and rule abstraction. Cognitive Neuropsychiatry, 6, 175192.

Morris, R.G., Rushe, T., Woodruffe, P.W.R., \& Murray, R.M. (1995). Problem solving in schizophrenia: A specific deficit in planning ability. Schizophrenia Research, 14, 235-246.

Pantelis, C., Barnes, T.R.E., Nelson, H.E., Tanner, S., Weatherley, L., Owen, A.M., \& Robbins, T.W. (1997). Frontal-striatal cognitive deficits in patients with schizophrenia. Brain, 120, 1823-1843.

Sabbe, B.G.C., Van Hoof, J.J.M., Hulstijn, W., \& Zitman, F.G. (1996a). Changes in fine motor retardation in depressed patients treated with fluoxetine. Journal of Affective Disorders, 40, 149-158.

Sabbe, B.G.C., Hulstijn, W., Van Hoof, J.J.M., \& Zitman, F.G. (1996b). Fine motor retardation in depression. Journal of Psychiatric Research, 30, 295-306.

Schatz, J. (1998). Cognitive processing efficiency in schizophrenia: Generalized vs domain specific deficits. Schizophrenia Research, 30, 41-49. 
Movement planning deficits in schizophrenia: Failure to inhibit automatic response tendencies | 125

Shallice, T. (1982). Specific impairments in planning. Philosophical transactions of the Royal Society of London series B Biological sciences, 298, 199-209.

Thomassen, A.J.W.M., \& Tibosch, H.J.C.M. (1991). A quantitative model of graphic production. In: G.E. Stelmach \& J. Requin (Eds.), Tutorials in motor neuroscience (pp. 269-282). Dordrecht: Kluwer Academic Publishers.

Thomassen, A.J.W.M., Meulenbroek, R.G.J., \& Tibosch, H.J.C.M. (1991). Latencies and kinematics reflect graphic production rules. Human Movement Science, 10, 271-289.

Van Galen, G.P. (1991). Handwriting: Issues for a psychomotor theory. Human Movement Science, 10, 165191.

Van Hoof, J.J.M., Hulstijn, W., Van Mier, J.I.A.J., \& Pagen, M. (1993). Fine drawing and psychomotor retardation: Preliminary results. Journal of Affective Disorders, 29, 263-266.

Van Hoof, J.J.M., Jogems-Kosterman, B.J.M., Sabbe, B.G.C., Zitman, F.G., \& Hulstijn, W. (1998). Differentiation of cognitive and motor slowing in the Digit Symbol Test (DST): Differences between depression and schizophrenia. Journal of Psychiatric Research, 32, 99-103.

Van Hoof, J.J.M. (2002). The abnormal development of drive and guidance mechanisms in the brain: The pathogenesis of schizophrenia. Acta Neuropsychiatrica, 14, 134-146.

Van Mier, H., \& Hulstijn, W. (1993). The effects of motor complexity and practice on initiation time in writing and drawing. Acta Psychologica, 84, 231-251.

Van Sommers, P. (1989). A system for drawing and drawing-related neuropsychology. Cognitive Neuropsychologica, 6, 117-164.

Vinter, A. (1993). Hierarchy among graphic production rules: A developmental approach. In: C. Faure, P. Keuss, G. Lorette \& A. Vinter (Eds.), Advances in handwriting and drawing: A multidisciplinary approach (pp. 275-291). Paris: Europia.

Watanabe, A., Kasai, K., Nagakubo, S., Kamio, S., Murakami, T., Hata, A., Iwanami, A., Nakagome, K., Fukuda, M., \& Kato, N. (2002). Verbal and procedural memory in schizophrenia with milder symptoms: Implications for psychosocial intervention. Schizophrenia Research, 53, 263-265.

Widlöcher, D., \& Hardy-Bayle, M.C. (1989). Cognition and control of action in psychopathology. European Bulletin of Cognitive Psychology, 9, 583-615.

Wykes, T., Katz, R., Sturt, E., \& Hemsley, D. (1992). Abnormalities of response processing in a chronic psychiatric group. A possible predictor of failure in rehabilitation programmes? British Journal of Psychiatry, 160, 244-252.

Wykes, T. (1994). Predicting symptomatic and behavioural outcomes of community care. British Journal of Psychiatry, 165, 486-492.

Wykes, T., Reeder C., \& Corner, J. (2000). The prevalence and stability of an executive processing deficit, response inhibition, in people with chronic schizophrenia. Schizophrenia Research, 46, 241-253. 


\section{Chapter 8}

\section{General discussion}

In the introduction of the present thesis (Chapter 1) it became apparent how important motor control and action planning are for understanding the behavior of patients with schizophrenia. There are numerous indications for the presence of psychomotor deficits in schizophrenia when observed in clinical psychiatry. Here, the term 'psychomotor' refers to a broad scale of human actions involving both cognitive and (sensori)motor processes. Deficits can be seen in a wide variety of everyday activities, ranging from a lack of initiative in performing actions (poverty of action) to stereotypical behavior (repeating actions purposelessly), or even chaotic or disorganized behavior (Liddle, 1987; Frith, 1992). Although these psychomotor deficits may cause a serious disruption of the daily life of the patient, they are rarely a major topic of research. In general, tests for psychomotor ability include a hotchpotch of tasks that vary considerably in the processes involved. Some of these tasks draw in particular on the ability to control motor actions (like tapping and tracing lines), whereas others make a greater appeal to more cognitive processes, such as working memory and attention (like the Digit Symbol Test and the Trailmaking Test). In addition, terms like 'cognitive' and 'motor' slowing are often used to describe certain psychiatric conditions, but unfortunately they induce no more than an artificial distinction in the compound of processes involved. The major aim of the present thesis was to investigate aspects of psychomotor deficits in schizophrenia. Its purpose was not to find a final answer to the question how these psychomotor deficits arise, but rather to examine some of the processes involved, particularly those that are required for controlling motor actions.

In the same introductory chapter, two theories on psychomotor deficits were more or less contrasted. The first puts forth that in schizophrenia there is an impairment in (sensori)motor or basic psychomotor processes, which, in addition, hinders the execution and learning of more complex skills (King, 1991); the second proposes that the problem lies primarily in the planning and organization of complex self-generated behavior (Widlöcher \& Hardy-Bayle, 1989; Frith, 1992; Liddle, 1993). The central questions in the present general discussion can thus be formulated as follows: what evidence do we have of psychomotor slowing, and, secondly, of more specific planning deficits in schizophrenia? Another important question that we try to answer here is whether and how this psychomotor slowing and these planning deficits relate to clinical symptoms of schizophrenia. First, an overview will be given of the major findings of the studies reported in the preceding chapters. Subsequently, we will discuss our major conclusions in the context of existing theories of schizophrenia. Finally, both implications for clinical practice and future research are given. 
Analyses of the Digit Symbol Test (DST) show a very differentiated picture of performances in patients with schizophrenia, which was found to be at least partly dependent on the severity of clinical symptoms

In neuropsychological research, measures of so-called 'psychomotor' or general speed of cognitive and motor processing mostly encompass numerous processes. An example of such a test is the Digit Symbol Test (DST), a short subtest of the Wechsler Adult Intelligence Scale (WAIS; Wechsler, 1956). In this test, subjects have to match digits with symbols, as quickly as possible, according to a reference key given at the top of the form. The DST does not only require sensorimotor processes, but also other more cognitive processes like visual search, working memory, and sustained attention. In the study of Chapter 2, we compared DST performances of patients with schizophrenia with those of depressed patients and controls. When compared to the group of healthy controls, both patient groups performed worse, i.e. both had significantly lower raw scores, on this test. A decomposition of the different performance elements (in Chapter 2), however, demonstrated that the type of slowing in schizophrenia differed from the slowing that could be observed in depression. The patients with schizophrenia were significantly retarded in the matching of the digits, which mainly requires cognitive processing, but not in writing speed, which mainly entails motor control. The depressed patients, on the other hand, were not only slower in finding the corresponding digit, but demonstrated an additional slowing in writing time. In general, these findings suggested at the time of that study that in schizophrenia cognitive speed is more affected than motor speed, which was found in agreement with a previous study of Nelson et al. (1990). The results of the depressed patients reconfirmed earlier findings of a manifest cognitive and motor slowing in depression (Van Hoof et al., 1993; Sabbe et al., 1996a, 1996b, 1997).

The DST was also employed in the study of Chapter 4. The performances of the patients with schizophrenia participating in this study showed a similar profile when compared to the patients from the study of Chapter 2. Compared to a healthy control group, these patients had lower raw scores and longer mean matching times per digit, but no longer mean writing times.

In addition to these earlier results, the study of Chapter 6 revealed an interesting difference between two subgroups of patients with schizophrenia. These subgroups consisted of one subgroup of patients selected by their psychiatrist to move to a residence outside the psychiatric institute and another subgroup of patients that was to remain hospitalized. Although these two subgroups did not differ with respect to raw score and mean matching time per digit, there was a significant difference in writing time in favor of the potential outpatient group.

When patient groups are ordered according to the type of setting in which they were staying, a rather consistent picture evolves of the DST performance as assessed in our series of studies. From the four schizophrenia groups, these two groups consisting of patients who received long-term inpatient care seem to perform most poorly on the DST. These lower raw scores were particularly associated with longer matching times. In addition, the schizophrenia group that participated in the study reported in Chapter 4 appears to perform relatively well when compared to the other three schizophrenia groups. This patient group consisted for the greater part (approximately 80\%) of patients living in a rather stable condition outside the psychiatric institute. Those patients from the study reported in Chapter 6 who were selected to leave the hospital, seem to perform somewhere 
between the two inpatient groups and the outpatient group. This was particularly true for raw score and matching time.

Do we have to conclude that our initial conclusion of a distinct type of slowing in schizophrenia and depression (Chapter 2) is not justified? This overview of our findings indeed suggests a more differentiated picture. In this perspective, it is worthwhile to examine the role of psychopathology. There are indications that schizophrenia can even be characterized by a depressive-like slowing (Holthausen et al., 1999; Brébion et al., 2000). Brébion et al. (2000), for example, found that in a group of patients with schizophrenia poor DST performances were related to depressive symptoms (as assessed by means of the Hamilton Depression Scale). At first, these findings seem to contrast with the results of two earlier studies (Nelson et al., 1990; Wolkin et al., 1992) in which a relationship was found between DST scores and negative symptoms. However, both these studies did not cover the full range of negative symptoms but used rating scales that assessed in particular the 'affect' part of these symptoms, like flatness of affect and mute (Nelson et al., 1990) or emotional blunting (Wolkin et al., 1992). Do our studies also suggest a relationship between DST performances and depressive-like symptoms? Our first DST study (Chapter 2) did not address the possible relationship between psychopathology and DST performance. In the study of Chapter 4, there was a tendency that cognitive processing speed (matching time in the DST) was related to self-reported depressive symptoms but not with negative symptoms, which seems to support Brébion et al.'s (2000) notion of a 'depressive slowing' in schizophrenia. However, in the total group of 64 patients in the study of Chapter 6, no indications were found of such a relationship. In contrast, we found a significant correlation between matching time (and raw score) and clinically observable symptoms of cognitive disorganization. Brébion et al. (2000), unfortunately, did not examine this relationship, but only focused on the observable negative and depressive symptoms. Perhaps patients with more severe features of cognitive disorganization will particularly have problems with the cognitive or visual-scanning efficiency that is required for optimal performance in the DST (c.f. Joy, Fein \& Kaplan, 2003)? Therefore, we can conclude that the relationship between clinical symptoms of schizophrenia and DST performance appears to be far more complex than a 'depressive slowing' would suggest; particularly cognitive inefficiency might cause impaired DST performance. To what extent poor DST performance is related to depressive symptoms (c.f. Brébion et al., 2000) or to disorganized symptoms deserves further exploration.

At least two other important subject characteristics could have influenced the observed performances on the DST. First, the length of hospitalization or treatment period may be related to the level of performance. It is reasonable to assume that the inpatients that had participated in our studies had a longer history of hospitalization or treatment periods, which could have negatively influenced their performances. How this relationship emerges cannot be answered now: does poorer general speed performance somehow influence the decision to hospitalize a patient or not, or is this rather the result of hospitalization? A study by our own research group in which both inpatients and outpatients are followed during the first two years after dehospitalization (Fakkers et al., 2002) will hopefully yield a better insight. Second, psychopharmacological medication may affect performance on the DST. Antipsychotic medication is known to influence cognitive and motor 
function, either positively (e.g., by improving attention) or negatively (e.g., by causing sedation or extrapyramidal motor side effects) (see King \& Green, 1996). All our patients with schizophrenia were using antipsychotic medication. We therefore cannot exclude the possibility that differences in medication regimes have affected the performance on the DST in our patient groups.

In conclusion, the results of our first DST study suggested at the time that schizophrenia is particularly associated with cognitive slowing, whereas in depression an overall slowing of both cognitive and motor slowing was observed (Chapter 2). Later observations (Chapters 4 and 6) in several groups of patients with schizophrenia across different types of settings, however, implied a more complex and differentiated picture. First, DST performance may at least be related to the severity of clinical symptoms, in particular to cognitive disorganization and to some extent to depressive features. Apart from this clinical manifestation, however, there were clear indications for psychomotor slowing, i.e. in the more strict sense (as measured by means of 'writing time'), in a subgroup of patients with schizophrenia. To understand the features of psychomotor slowing in this subgroup, further exploration is certainly needed. Moreover, based on the overview of DST performance throughout the present thesis, it might be concluded that writing time, as a measure of psychomotor slowing, constitutes an oversimplistic representation of processes involved. First, the duration of writing characters (like the digits in the DST) is dependent on the size of the production and the acceleration of the movements (e.g., Chapter 4). Second, the preparation required for writing characters may also demand part of the matching time. A similar problem arises when restricting matching time to being a measure of cognitive processing. In searching the right character, also (sensori)motor processes are likely to be involved; for example in moving the eyes.

As a final remark, thanks to some effort we were able to draw an overall picture of DST performance in several groups of patients with schizophrenia. However, this endeavor may seem illustrative for the complexity of both psychomotor and cognitive ability in schizophrenia.

Clinical signs of drug-induced slow moving ('bradykinesia') are related to slowing on simple psychomotor tasks rather than complex tasks

Psychomotor performance in schizophrenia is conceivably influenced by the use of antipsychotic drugs. These drugs, and particularly the more conventional ones, are known to induce a number of undesirable motor side effects, the so-called extrapyramidal symptoms. One of these is the slowdown of gross and fine motor activity: bradykinesia. Patients with schizophrenia may thus demonstrate, for instance, a clearly retarded gait. Both from a theoretical viewpoint (is the slowing disease-related or due to the medication?) and a therapeutical point of view (what are the effects of administering or changing medication?), it is of interest to quantify this motor retardation. The study of Chapter 3 can be viewed as a first attempt to relate clinical measures of bradykinesia in schizophrenia with the temporal and spatial features of fine motor movements. The results of this study revealed that the clinical scores of bradykinesia, as assessed by means of the SADIMoD (Loonen et al., 2000, 2001), correlated with simple movement variables but not with more complex ones. For example, higher scores on bradykinesia were associated with longer initiation times in the copying of simple letters but not of more complex and unfamiliar patterns. It is very likely that in copying more complex patterns poorer performance merely reflects possible cognitive deficits. 
Other significant correlates of bradykinesia were found in a goal-directed line-drawing task, i.e., initiation time before the start of the movement and pause duration during the execution phase. In general, the results of the study reported in Chapter 3 suggest that analyses of writing and drawing movements made during simple psychomotor tasks can be very helpful in the quantification of the severity of bradykinesia as might be induced by antipsychotic medication.

Although psychomotor slowing is a significant aspect of schizophrenia, specific planning deficits can be observed, particularly in those patients with severe negative symptoms

Robust evidence for psychomotor slowing in schizophrenia is, undoubtedly, provided by the study described in Chapter 4. Compared to a control group, the patient group participating in this study was even retarded in the copying of simple lines. In addition, when compared to these healthy controls, the patients were equally retarded (about one-third) in their performance time on all three tasks that were applied in the study (the DST, the simple line-copying task and a more complex figure-copying task), which suggests a more or less general slowing. The expectation that the patients with schizophrenia would exhibit slowing mainly on those variables that reflect cognitive processing (cf. Nelson et al., 1990), such as initiation time, reinspection time and duration of pauses during execution in the copying tasks, and matching time in the DST, was to a great extent confirmed. Initiation time in the copying tasks was significantly longer in the patient group. Furthermore, patient performance showed significantly longer periods above the paper (pen-up time), and significant slowing in reinspection time, variables also mainly associated with cognitive processing. In general, patients did not show lower movement velocity, which provides support for the absence of motor slowing. Not fully in line with the notion of a predominantly 'cognitive' slowing were the longer movement times in the patients with schizophrenia. These findings may point to a more basic problem with fine motor control: the patients tended to write and draw larger, without being slower than the controls. By either increasing or decreasing the size of the writing or drawing, a person might compensate for the problems he or she experiences with fine motor control (cf. Gallucci et al., 1997). Whether this compensation mechanism relates to the use of antipsychotic medication or not, must be addressed in future follow-up studies. In our study, no relationship was found between the different movement variables and the dosage of the antipsychotic agents.

In the study of Chapter 4, it was further shown that, when stimuli to be copied were more complex and/or less familiar, patients with schizophrenia reexamined the patterns longer while copying as compared to the controls. This finding suggests that they were less able to plan and memorize the required movements before starting drawing. The expected prolongation of initiation times was not found in the patient group as a whole, but only in a subgroup of patients with higher scores on negative symptoms. Moreover, patients with higher negative symptom scores demonstrated not only longer initiation and reinspection times, but also more pausing (i.e. decision) time, during the copying of the more complex patterns. It was, therefore, suggested that planning problems were most striking in this subgroup of patients with more severe negative symptoms: despite their longer initiation time they were least able to plan most of the required movements before copying. 
(Future) outpatients with schizophrenia seemed less sensitive to conflicting graphic production rules, which may be due to a lack of planning and/or to the use of a more automatic or stereotyped response pattern

A major disadvantage of the complex figure-copying task (Chapter 4) for the use of a 'planning' task is that it makes a very strong appeal to one's working memory capacity. Particularly when asked to copy unfamiliar patterns consisting of eight line-segments, a person might find it difficult to put this pattern in a temporary memory store and to hold its information on-line during the copying phase. To reduce the influence of a restricted working memory capacity, we also examined planning ability by using copying tasks in which the stimuli to be copied consisted of not more than two or three line-elements (Chapters 5,6 and 7). This type of tasks is based on the assumption that our graphic behavior is governed by certain implicit preferences, the so-called graphic production rules (Thomassen \& Tibosch, 1991; Thomassen, Meulenbroek \& Tibosch, 1991). These rules incorporate preferences with respect to starting points, stroke directions and stroke sequences. In copying certain patterns (like the letter L), these graphic production rules can be more or less easily applied. However, the copying of other patterns (for example when such patterns are mirrored around the Y-axis) will confront the subject with conflicts between rules. It is assumed that for the copying of non-conflicting (NC) patterns a routine or automatic strategy suffices, whereas the copying of conflicting (C) patterns requires the planning of the most suitable sequence of lines (Thomassen, Meulenbroek \& Tibosch, 1991). The amount of experienced conflicts will normally influence the speed of copying. In a group of healthy subjects, copying 'conflicting' patterns resulted in somewhat longer latencies before the start of the drawing and with significantly longer hesitations above the paper during execution (Thomassen, Meulenbroek \& Tibosch, 1991). The hypothesis of the study in Chapter 5 was that because of a lack of planning (Frith, 1992; Widlöcher \& Hardy-Bayle, 1989) patients with schizophrenia would show fewer differences between copying $\mathrm{NC}$ and $\mathrm{C}$ patterns. The results generally confirmed these predictions, in that differences between $\mathrm{NC}$ and $\mathrm{C}$ patterns were found in a healthy control group, and even more strongly in a depression group, but not in the schizophrenia group.

Reliance on an automatic or stereotyped strategy was particularly found in patients that did not exhibit features of psychomotor slowing.

The schizophrenia patients from Chapter 5 participated in a larger study on the predictive value of cognitive functions for the future outcome of patients (Fakkers et al., 2002), and they were, at the moment of testing, all selected to leave the psychiatric institute. The results of the earlier study described in Chapter 5 were not confirmed by later observations in other groups of patients with schizophrenia (Hulstijn et al., 2001). In these groups, all of which consisted of hospitalized patients, differences were found between the copying of $\mathrm{NC}$ and $\mathrm{C}$ patterns. In this respect, paradoxically, the performances of these inpatients resembled those of the depressed patients more than those of the future schizophrenia outpatients. To clarify these results, we decided to compare performance of the (future) outpatients with those of a group of inpatients directly (Chapter 6). Both these groups were participating in the same larger predictive study (Fakkers et al., 2002). As the performances of the inpatients paralleled more strongly those of the depressed patients, it could be expected that 
these inpatients would exhibit more severe psychopathology and particularly more depressive symptoms. However, contrary to these expectations, the results of the study showed that the inpatient group did not differ from the outpatient group with respect to clinical ratings. Thus, the finding that the inpatients exhibited larger differences between the $\mathrm{NC}$ and $\mathrm{C}$ patterns could not be attributed to a more severe psychopathology or to the number or severity of depressive features. Another possible explanation was that the larger differences between NC and C could be attributed to a psychomotor slowing that may underlie symptoms of schizophrenia, and particularly the accompanying depressive symptoms. In two previous studies, it was found that some measures of 'psychomotor' speed (DST; Brebion et al., 2000) (tapping; Holthausen et al., 1999) correlated with the severity of depressive symptoms in patients with schizophrenia. The results of our study reported in Chapter 6 indeed showed that larger differences between $\mathrm{NC}$ and $\mathrm{C}$ were related to a stronger psychomotor slowing (as measured particularly by a goal-directed line-drawing task). However, the assumption of a depressive-like slowing in schizophrenia (Brébion et al., 2000; Holthausen et al., 1999) could not be supported since we did not find significant correlations between our measures of psychomotor speed and severity scores for depression. It should, however, be mentioned again that comparing our data with those of others is complicated by the fact that the term 'psychomotor' is often used very differently. Whereas Holthausen et al. (1999) used a tapping task as a measure of psychomotor speed, Brébion et al. (2000) took the DST for this purpose. In the study of Chapter 6, we reported on the contrary a strong correlation between DST performance and clinical ratings of cognitive disorganization. In general, we still share Brébion et al's (2000) opinion that, although psychomotor slowing and depression might be related in at least a number of patients, they may act as independent factors as well.

Taken collectively, the results of the study reported in Chapter 6 could be explained meaningfully by assuming two types of planning problems in schizophrenia. Patients who do not show features of psychomotor slowing might exhibit a stronger reliance on an automatic or stereotyped strategy when task demands are in conflict: they just follow the most dominant rule and seem not to anticipate consequences of conflicting rules. When, on the other hand, patients show psychomotor slowing, they might experience these planning conflicts in about the same way as depressed patients do.

The question remains why this first cluster of patients, without features of psychomotor slowing, were particularly represented in the (future) outpatient group. Is the absence of a psychomotor slowing in some way related to the selection of patients to leave the psychiatric institute? As both groups of patients were followed during the succeeding two years (Fakkers et al., 2002), these follow-up data will hopefully shed light on the possible predictive value of this psychomotor slowing for the functional outcome of patients.

Whereas patients with more severe negative symptoms tended to be slower when asked to start copying at a particular point, patients with clinical features of cognitive disorganization appeared to be faster in this condition

Previous studies have suggested that planning difficulties in schizophrenia are related to an inability to inhibit strongly cued automatic responses (e.g., Marczewski, Van der Linden \& Larøi, 2001; 


\section{4 | Chapter 8}

Barch \& Carter, 1998; Everett, Laplante \& Thomas, 1989). Our finding that patients did not show the expected prolongation of initiation time in the more complex task conditions (Chapter 4) could also be discussed from this viewpoint. The study of Chapter 7 tried to address this failing ability to inhibit well-learned preferences. Specifically, the goal of the study was to assess whether patients have difficulty with (1) the initiation and planning of simple drawing movements (that do not challenge the capacity of working memory), and (2) the inhibition of applying highly preferred graphic production rules. The results showed that patients were not slower than controls in copying lines or simple L-shaped patterns. However, when the position of the L's (or reversed L's) was varied in the small work field ('drawing box'), patients needed more initiation time to copy the patterns. More detailed analyses suggested that these longer latencies did not reflect problems with visual-spatial planning, but rather a difficulty with deviating from a normal habitude to draw in the middle of the box. Furthermore, when they were forced to start their drawing from a less preferred point (indicated by a black dot in the pattern itself), the patient group as a whole showed longer initiation times as compared to the controls. This prolongation of the initiation time, however, tended to be greater particularly for patients with predominantly negative symptoms. Patients showing features of cognitive disorganization appeared even to react faster in this condition (irrespective of whether the dot was located at a highly preferred starting point or not), which is consistent with the explanation of Frith (1992) that the actions of these patients are excessively stimulus-bound.

In general, the findings of the study described in Chapter 7 suggested that difficulties with inhibiting an automatic response tendency play a crucial role in the planning problems of patients with schizophrenia. Theoretically, the selection of an action can be viewed as a competitive process (Control of Action Model; Shallice, 1988). The expected prolongation of initiation time in the schizophrenia group when the starting dot opposed a strongly preferred starting point might be seen as a resultant of a lack of flexibility in controlling their actions. That this process is time-consuming is particularly seen in patients with negative symptoms. Patients with cognitive disorganization react relatively fast, because they seem to focus more strongly on the objective stimulus information (the starting dot) rather than the contextual stimulus information (the starting dot in relation to the preferred starting point).

\section{Final conclusions}

On the surface, schizophrenia seems to be heterogeneous (Andreasen, 1999). It was indeed a recurring feature in the present thesis that in patients with schizophrenia 'psychomotor' ability could differ on so many dimensions, even when performing relatively simple fine motor tasks.

Our first exploration of psychomotor slowing in schizophrenia started by using the Digit Symbol Test (DST), in which we additionally tried to differentiate the contribution of cognitive and motor processes. Although the use of the DST for assessing 'psychomotor' speed can be criticized because of the compound of processes involved, it is one of the most frequently applied tests for general cognitive and motor processing speed in neuropsychological research. An exposition of DST performance throughout several of our studies (Chapters 2, 4 and 6) seemed to corroborate the multiple reasons for why patients may perform worse. Our attempt at differentiating between 
'cognitive' and 'motor' slowing could not fully solve this. Although our initial study (Chapter 2) suggested a distinct type of slowing in schizophrenia (mainly 'cognitively') as compared to depression (a diffuse slowing), this conclusion could not be maintained in the study of Chapter 6. It appeared that psychomotor slowing, i.e. in the more strict sense, may be present in at least a subgroup of patients with schizophrenia. In search of factors that could explain differences in DST performance between patient groups, it revealed that at least the severity of clinical symptoms played a significant role with respect to the more 'cognitive' component of the test. Particularly patients with schizophrenia that showed clinical features of cognitive disorganization appeared to perform worse (Chapter 6). The possible relationship with depressive symptoms (Brébion et al., 2000) could not be confirmed unequivocally by our data. In this line, it is fair to conclude that the DST requires cognitive processing that goes beyond what might be expected from a test of psychomotor performance. Although the test is highly sensitive to neuropsychological functioning (Lezak, 1995), too many different impairments in cognitive processing are conceivable that may impede performance (e.g., Joy, Fein \& Kaplan, 2003). Consequently, we recommend not using the term 'psychomotor' when there are so many cognitive processes involved. Therefore, in most of the studies reported in the present thesis, it was tried to develop fine motor tasks from a cognitive neuropsychological viewpoint (e.g., Parkin, 1996) that would be more sensitive for psychomotor ability.

In addition to the DST, the results of simple (sensori)motor tasks have demonstrated that psychomotor slowing can be observed in a significant proportion of the patients with schizophrenia (c.f. King, 1991). Particularly in the study of Chapter 4, patients with schizophrenia were slower in the copying of single lines when compared to normal controls. Yet, the results of Chapter 7 seem illustrative for the fact that psychomotor slowing does not occur in all patients with schizophrenia. In this study, patients with schizophrenia appeared not to be significantly slower in copying single lines and/or L-shaped patterns as compared to their control group. As far as the implication of the different studies of the present thesis stretches, no relationship is found between the expression of psychomotor slowing and the clinical symptoms of schizophrenia. Therefore, it may be argued that psychomotor slowing can occur as a non-specific phenomenon of schizophrenia. The results of Chapters 5 and 6 , nevertheless, suggested an interesting difference between patients showing features of psychomotor slowing and patients that did not. Those patients that were not slowed down on a simple goal-directed line-drawing task, which could be seen as indicative for psychomotor speed, tended to use a more automatic and stereotyped way of copying L-shaped patterns. This may point to an interesting qualitative difference between patients that show psychomotor slowing and those that do not. Whether it constitutes a manifest distinction between subgroups of patients with schizophrenia needs to be addressed in future research.

The results of performances on more complex figure-copying tasks suggested distinct planning deficits in patients with schizophrenia. When compared to normal controls, patients were not only slower in copying figures varying in number of line elements and/or familiarity (Chapter 4), but also needed more initiation time when tasks demanded deviation from a general tendency to draw a pattern in the middle or to start drawing at a particular point (Chapter 7). Using these figurecopying tasks, we were able to demonstrate a specific deficit in more complex planning processing, 
which evolved over and above a slowing on simple copying tasks. This deficit accounted for an extra slowing in patients with more severe negative symptoms. Patients with features of cognitive disorganization, on the other hand, appear to compensate this shortfall by concentrating more strongly on certain stimulus features (like the starting dot in our experiment).

According to King (1991), psychomotor performance in schizophrenia patients might be just as discriminative from healthy subjects as their performance on more complex cognitive tasks. Initially, the results of Chapter 4 pointed in this direction, because the patient group as a whole was about one-third slower in their performance as compared to the control group, independent of the complexity of the task. However, based on further findings in the present thesis (Chapters 4 to 7 ), it is concluded that tasks that challenge in particular planning ability seem more sensitive to variations within the population of patients with schizophrenia. This can be considered to be in line with the theory of Frith (1992). According to his 'Willed Action' model, patients with schizophrenia have difficulties particularly with the generation and planning of self-motivated actions, whereas they are less impaired in reactions that are fully triggered by external stimuli. These problems with selfgenerated actions are supposed to lead either to a poverty of action (in which goals fail to initiate action) or to an inability to suppress inappropriate actions (in which goals fail to inhibit stimulusdriven actions). The finding that patients with schizophrenia showing negative symptoms were slower in the planning and execution of copying complex patterns (Chapter 4) might be viewed as an expression of this poverty of action, as proposed by Frith (1992). Moreover, the results of Chapter 7, showing that patients with clinical features of cognitive disorganization were triggered by an imposed starting point might be considered as an example of the inability to suppress stimulus-elicited behavior (Frith, 1992). However, as has also been shown in the present thesis, some patients with schizophrenia might even be retarded in simple psychomotor tasks, which is in agreement with King's ideas. At present, it may therefore be justified to acknowledge that the ideas both of King (1991) and of Frith (1992) cannot fully explain the complex nature of schizophrenia, but at least help to put the (two, three or even more?) pieces of a puzzle together.

But how does the knowledge that psychomotor deficits may be present in schizophrenia fit in the current thinking about the illness? Whereas most of the contemporary models (Andreasen, 1999; Goldman-Rakic, 1994; Braff, 1993; Frith, 1992) still emphasize the cognitive nature of schizophrenia, in recent years there is a growing consideration that motor control may play a more prominent role in the concept of the illness. Recently, Andreasen (1999) proposed that 'cognitive dysmetria' being the counterpart of 'motor dysmetria' constitutes the core problem in schizophrenia. According to this model, patients with schizophrenia are assumed to have difficulties particularly with the smooth planning and execution of both cognitive and motor actions. Considering not only the results of the present studies, but also those of previous ones (e.g., Braff et al., 1991; Bilder et al., 2000), it seems legitimate to state that 'psychomotor function' deserves a separate position in the list of neuropsychological impairments associated with schizophrenia (see Figure 1 on page 15 in the general introduction). Stated differently, psychomotor slowing cannot be seen as solely resulting from problems in one or more of the other neuropsychological domains (like executive function, attention or memory), but it forms an entity in itself. 
Although it is important to acknowledge that psychomotor deficits are essentially part of the behavior of patients with schizophrenia, it does not mean that they express themselves in a uniform manner. As has been made clear in the present studies, they can be manifested in several ways. First, psychomotor deficits may arise because of problems with the forming of a goal or plan for a particular motor action. Second, there might be a problem with the perceptual motor integration necessary to select the target for moving. Third, a person might not be able to put the different movements in the right sequence. Finally, there could be a difficulty with achieving the right muscle activity. These four processes and the way in which they are engaged in motor actions have been described in more detail by Willingham (1998). More than was elaborated in the present studies, future research should focus on the role that impairments in one or more of these processes play in psychomotor behavior in patients with schizophrenia. Here lies as a particular challenge the analysis of the strategies or plans that subjects have, or fail to have, in performing actions. Willingham (1998) already mentioned the difficulty of discovering strategies that are adopted by subjects in experimental test conditions, not to mention in daily life. To exemplify, according to Frith (1992) poverty of action (or 'psychomotor' poverty) arises when goals fail to generate intentional behavior. This formulation implies that patients with overt signs of psychomotor poverty do have plans, yet are not able to convert them into action. But what could be the origin of this failure? It might be that, in addition to problems with the planning of actions, a subject just misses the necessary 'drive' or 'power' to fulfill these. This notion stems from a 'progressive' neurodevelopmental model that has recently been put forward by Van Hoof $(2002,2003)$. Its origin was indeed inspired for a great part by the data presented throughout the present thesis. According to this model, schizophrenia is the manifestation of an imbalance between two motivational mechanisms in the brain which are responsible, respectively, for initiating and dosing ('drive'), and for planning and inhibition ('guidance'). During the development of the brain, both these mechanisms are thought to be implemented in a repetitive way from the "how to do" motor domain into the "what to do" intentional domain. It is further assumed that the "drive' mechanism (which is mainly located in the ventral part of the brain) functions insufficiently in individuals that are to develop schizophrenia. In addition, an exaggerated reliance on, and the eventual failing of the 'guidance' mechanism (which is mainly located in the dorsal part of the brain) could, however, activate this 'drive' mechanism. This will result in a disinhibition of activity. However, when the 'drive' mechanism is failing too, this would manifest itself as psychomotor poverty. Actually, the model might better explain why 'higher order' planning and 'lower order' psychomotor disturbances could both be present in schizophrenia. In addition to other theories (e.g., Frith, 1992; Andreasen, 1999), Van Hoof's model and the different predictions that are to be derived from it constitute another target for future research.

\section{Clinical implications}

Without doubt, a deeper insight into the nature of psychomotor deficits in schizophrenia will help to understand the problems that patients have to deal with in daily life. It might be useful to figure out why patients are unable to plan and/or execute certain daily life activities. At the level of intervention or treatment, a psychiatrist or other therapist (e.g., psychologist and social worker) has 
to know upon which aspect the therapy should be focused. Until now, a significant part of the diagnostic process is based on self-reports and observation of the patients rather than on clear-cut test results. Here, the assessment of psychomotor performance could be helpful firstly in objectifying these reports, and secondly, in investigating the nature of the disease, because it goes beyond the description of symptoms (Withaar \& Arends, 2002). Our patients also consider psychomotor ability as important. They describe problems with attending to simple everyday tasks, not only at the time of an acute exacerbation of the illness but also between such episodes.

By using a cognitive neuropsychological research method in which figures had to be copied under concomitant accurate digital recording of pen movements, it was demonstrated that psychomotor slowing constitutes an important feature of schizophrenia. This slowing may have important implications for understanding the sources of dysfunction in schizophrenia (cf. Mohamed et al., 1999; Van Beilen et al., 2002). In addition to this psychomotor slowing, there was evidence for more specific problems with planning, particularly when the task requirements were incompatible with a normal preference. In contrast to other planning tasks, such as the Tower of London (Shallice, 1982), our tasks do not challenge the conscious and 'intellectual' ability of decomposing the task into subgoals and putting these in the most appropriate order. On the contrary, they require implicit adjustments of behavior given the context of the task. As most of our daily activities like dressing, washing and other domestic activities are not governed by explicit (and more or less 'intellectual') rules but by implicit rules of which we are not aware, our type of tasks might better capture the actual problems that patients encounter in daily life. Usually, we have to make minor, but not unimportant choices and adjustments that are not explicitly planned either. Patients with schizophrenia are likely to be impaired in this 'flow' of choices and might have problems particularly in conflicting situations in which they have to change to another solution or to a more conscious control of action.

In recent years, impaired cognitive test performances have been found to be better predictors of the functional outcome of patients than clinical symptoms (Green, 1996; Green et al., 2000). Particularly impaired executive functioning, which includes volitional activity, forward planning, cognitive flexibility and self-regulation (following Lezak, 1995) have been found to be related to poor social, vocational and community functioning in the long term. Following this line of research we feel that the tasks used in our studies may be useful to understand cognitive deficits and problems in the everyday functioning of patients with schizophrenia in the perspective of the future outcome for these patients. This is also the purpose of a recent study of our own research group (Fakkers et al., 2002). Preliminary results show that psychomotor speed has more predictive value for functional outcome as has been highlighted in recent overviews (Green, 1996; Green et al., 2000). A number of earlier studies have indeed indicated that psychomotor measures have predictive value for the long-term daily living situation of patients with schizophrenia after release from a psychiatric hospital (Weaver \& Brooks, 1967; Buchanan et al., 1994; Goldman et al., 1993).

The importance of cognition in the disorder of schizophrenia has led to the optimistic thought that cognitive functions can be trained and that this would positively affect daily functioning. However, the results of cognitive training programs have demonstrated the persistence of cognitive deficits in patients with schizophrenia. Cognitive training results in no more than short- 
term improvements on the trained tasks (Van der Gaag, 1992; see also Van der Gaag et al., 1994). They do not hold over time and no generalization to every day functioning occurs (Wykes et al., 1999; Van der Gaag et al., 2002). Hence, cognitive deficits are increasingly regarded as "ratelimiting" factors, restricting the ability of patients to retain and acquire skills that are needed in daily functioning. Most of the current skill training programs therefore take the cognitive impairments into account (for a recent overview, see Withaar \& Arends, 2002). However, it would be interesting to examine whether improvement of psychomotor speed, which might be established by medication for instance, has a positive effect on the course and outcome of these skill-training programs.

\section{Future directions}

A first challenge for future research may be to extend the use of psychomotor tasks. This would be the only way to provide a fuller understanding of the role of psychomotor slowing in schizophrenia. Here, the development of adequate psychomotor tasks should follow current views on motor control. Willingham (1998), for example, offers a useful framework in which the different neuropsychological aspects of motor control (goal formation, perceptual motor integration, sequencing and selection of muscle commands) are described. By manipulating these aspects in experimental tasks, it would be feasible to investigate the role of each of these in the behavioral abnormalities of schizophrenia and other psychiatric diseases like depression.

A second line of research that is important to pursue is that of examining the relationship between psychomotor slowing and planning deficits, on the one hand, and clinical and functional outcome of patients, on the other. Although recent studies in this area (for an overview, see Green, 1996; Green et al., 2000) have particularly focused on the relationship between cognitive deficits and impairments in social and vocational functioning, less is known of the role of psychomotor slowing in this context.

A third topic warranting further investigation is the possible effect of psychotropic medication on psychomotor tasks. By randomly assigning medication to patients, and following these patients during the course of treatment, we will be able to examine with great accuracy any positive and/or negative effects of medication per se and to assess possible differences between various types of medication.

A fourth challenge for future research is to examine the relationship between psychomotor performance and planning ability and brain activity. Whereas there is a traditional focus on the role of the frontal lobe in the cognitive functioning of patients with schizophrenia (for detailed reviews see Taylor, 1996; Weinberger \& Berman, 1996), future studies should extend this focus by examining the contribution of sub-cortical and cerebellar structures in particular (e.g., CrespoFacorro et al., 1999).

Finally, a remark on the overall method used in the present thesis. As in so many other studies on schizophrenia, the selection of patients was based on standard clinical criteria for schizophrenia (according to DSM criteria). The fact that patients satisfy these criteria at least seem to imply that they form a homogeneous group. However, a recurring result in the present thesis was that the variation within groups of patients was quite large, which could only partially be explained 
140 | Chapter 8

by differences in clinical symptoms. What to do with this variability? Although future research on schizophrenia might ultimately point to a kind of unitary concept underlying the illness (e.g., Andreasen, 1999), for the time being, it would be recommendable to investigate the different signs and symptoms in their own right. This might prove an efficient approach to understanding why patients with schizophrenia act 'differently'. The studies of the present thesis have hopefully contributed to such understanding. 


\section{References}

Andreasen, N. (1999). A unitary model of schizophrenia. Bleuler's "fragmented phrene" as schizencephaly. Archives of General Psychiatry, 56, 781-787.

Barch, D.M., \& Carter, C.S. (1998). Selective attention in schizophrenia: Relationship to verbal working memory. Schizophrenia Research, 33, 53-61.

Bilder, R.M., Goldman, R.S., Robinson, D., Reiter, G., Bell, L., Bates, J.A., Pappadopulos, E., Wilson, D.F., Alvir, J.M.J., Woerner, M.G., Geisler, S., Kane, J.M., \& Lieberman, J.A. (2000). Neuropsychology of first-episode schizophrenia: Initial characterization and clinical correlates. American Journal of Psychiatry, 157, 549-559.

Braff, D.L., Heaton, R., Kuck, J., Cullum, M., Moranville, J., Grant, I., \& Zisook, S. (1991). The generalized pattern of neuropsychological deficits in outpatients with chronic schizophrenia with heterogeneous Wisconsin Card Sorting Test results. Archives of General Psychiatry, 48, 891-898.

Braff, D.L. (1993). Information processing and attention dysfunctions in schizophrenia. Schizophrenia Bulletin, 19, 233-259.

Brébion, G., Amador, X., Smith, M., Malaspina, D., Sharif, Z., \& Gorman, J.M. (2000). Depression, psychomotor retardation, negative symptoms, and memory in schizophrenia. Neuropsychiatry, Neuropsychology and Behavioral Neurology, 13, 177-183.

Buchanan, R.W., Holstein, C., \& Breier, A. (1994). The comparative efficacy and long-term effect of clozapine treatment on neuropsychological test performance. Biological Psychiatry, 36, 717-725.

Caliguiry, M.P., Lohr, J.B., \& Jeste, D.V. (1993). Parkinsonism in neuroleptic-naive schizophrenic patients. American Journal of Psychiatry, 150, 1343-1348.

Crespo-Facorro, B., Paradiso, S., Andreasen, N.C., O’Leary, D.S., Watkins, G.L., Boles Ponto, L.L., \& Hichwa, R.D. (1999). Recalling word lists reveals "cognitive dysmetria" in schizophrenia: A positron emission tomography study. American Journal of Psychiatry, 156, 386-392.

Everett, J., Laplante, L., \& Thomas, J. (1989). The selective attention deficit in schizophrenia: Limited resources or cognitive fatigue? Journal of Nervous and Mental Disease, 177, 735- 738.

Fakkers, G.P.F.M., Jogems-Kosterman, B.J.M., Loonen, A.J.M., Van Hoof, J.J.M., \& Hulstijn, W. (2002). Changes in clinical functioning and neurocognitive performances in patients with schizophrenia during the first years of deinstitutionalization. Schizophrenia Research, 53, 127.

Frith, C.D. (1992). The cognitive neuropsychology of schizophrenia. Hove: Lawrence Erlbaum Associates.

Gallucci, R.M., Philips, J.G., Bradshaw, J.L., Vaddadi, K.S., \& Pantelis, C. (1997). Kinematic analysis of handwriting movements in schizophrenic patients. Biological Psychiatry, 41, 830-833.

Goldman, R.S., Axelrod, B.N., Tandon, R., Ribeiro, S.C., Craig, K., \& Berent, S. (1993). Neuropsychological prediction of treatment efficacy and one-year outcome in schizophrenia. Psychopathology, 26, 122-126.

Goldman-Rakic, P.S. (1994). Working memory dysfunction in schizophrenia. Journal of Neuropsychiatry and Clinical Neurosciences, 6, 348-357.

Green, M.F. (1996). What are the functional consequences of neurocognitive deficits in schizophrenia? American Journal of Psychiatry, 153, 321-330.

Green, M.F., Kern, R.S., Braff, D.L., \& Mintz, J. (2000). Neurocognitive deficits and functional outcome in schizophrenia: Are we measuring the "right stuff"? Schizophrenia Bulletin, 26, 119-136. 
Hanes, K.R., Pantelis, C., Andrewes, D., \& Chiu, E. (1996). Subcortical dysfunction in schizophrenia: A comparison with Parkinson's disease and Huntington's disease. Schizophrenia Research, 19, 121-128.

Holthausen, E.A.E., Wiersma, D., Knegtering, R.H., \& Van den Bosch, R.J. (1999). Psychopathology and cognition in schizophrenia spectrum disorders: The role of depressive symptoms. Schizophrenia Research, 39, 65-71.

Hulstijn, W., Jogems-Kosterman, B.J.M., Wezenberg, E., Sabbe, B.G.C. (2001). An evaluation of the use of figure-copying tasks in studies of planning deficits in schizophrenia. In: R.G.J. Meulenbroek \& B. Steenbergen (Eds.), Proceedings of the tenth biennial conference of the International Graphonomics Society (pp. 46-51). Nijmegen: IGS.

Joy, S., Fein, D., \& Kaplan, E. (2003). Decoding Digit Symbol: Speed, memory, and visual scanning. Assessment, 10, 56-65.

King, H.E. (1991). Psychomotor dysfunction in schizophrenia. In: S.R. Steinhauer, J.H. Gruzelier \& J. Zubin (Eds.), Handbook of schizophrenia, Vol. 5: Neuropsychology, psychophysiology and information processing (pp. 273-301). Amsterdam: Elsevier Science Publishers.

King, D.J., \& Green, J.F. (1996). Medication and cognitive functioning in schizophrenia. In: C. Pantelis, H.E. Nelson \& T.R.E. Barnes (Eds.), Schizophrenia: a neuropsychological perspective (pp. 419-444). New York: John Wiley \& Sons.

Lezak, M.D. (1995). Neuropsychological Assessment (3rd edition). Oxford: University Press.

Liddle, P.F. (1993). The psychomotor disorders: Disorders of the supervisory mental processes. Behavioural Neurology, 6, 5-14.

Liddle, P.F. (1987). Schizophrenic syndromes, cognitive performance and neurological dysfunction. Psychological Medicine, 17, 49-57.

Loonen, A.J.M., Doorschot, C.H., Van Hemert, D.A., Oostelbos, M.C.J.M., Sijben, A.E.S., \& the MASEAS Team (2000). The Schedule for the Assessment of Drug-Induced Movement Disorders (SADIMoD): Test-retest reliability and concurrent validity. International Journal of Neuropsychopharmacology, 3, 285-296.

Loonen, A.J.M., Doorschot, C.H., Van Hemert, D.A., Oostelbos, M.C.J.M., Sijben, A.E.S., \& the MASEAS Team (2001). The Schedule for the Assessment of Drug-Induced Movement Disorders (SADIMoD): Inter-rater reliability and construct validity. International Journal of Neuropsychopharmacology, 4, 347360.

Marczewski, P., Van der Linden, M., \& Larøi, F. (2001). Further investigation of the Supervisory Attentional System in schizophrenia: Planning, inhibition and rule abstraction. Cognitive Neuropsychiatry, 6, 175192.

Malla, A.K., Norman, R.M.G., Aguilar, O., Carnahan, H., \& Cortese, L. (1995). Relationship between movement planning and psychopathology profiles in schizophrenia. British Journal of Psychiatry, 167, 211-215.

Mohamed, S., Paulsen, J.S., O’Leary, D., Arndt, S, \& Andreasen, N. (1999). Generalized cognitive deficits in schizophrenia: A study of first-episode patients. Archives of General Psychiatry, 56, 749-754.

Morris, R.G., Rushe, T., Woodruffe, P.W.R., \& Murray, R.M. (1995). Problem solving in schizophrenia: A specific deficit in planning ability. Schizophrenia Research, 14, 235-246. 
Nelson, H.E., Pantelis, C., Carruthers, K., Speller, J., Baxendale, S., \& Barnes, T.R.E. (1990). Cognitive functioning and symptomatology in chronic schizophrenia. Psychological Medicine, 20, 357-365.

Pantelis, C., Barnes, T.R.E., Nelson, H.E., Tanner, S., Weatherley, L., Owen., A.M., \& Robbins, T.W. (1997). Frontal-striatal cognitive deficits in patients with schizophrenia. Brain, 120, 1823-1843.

Parkin, A.J. (1996). Explorations in cognitive neuropsychology. Oxford: Blackwell Publishers.

Sabbe, B.G.C., Van Hoof, J.J.M., Hulstijn, W., \& Zitman, F.G. (1996a). Changes in fine motor retardation in depressed patients treated with fluoxetine. Journal of Affective Disorders, 40, 149-158.

Sabbe, B.G.C., Hulstijn, W., Van Hoof, J.J.M., \& Zitman, F.G. (1996b). Fine motor retardation in depression. Journal of Psychiatric Research, 30, 295-306.

Sabbe, B.G.C., Van Hoof, J.J.M., Hulstijn, W., \& Zitman, F.G. (1997). Depressive retardation and treatment with fluoxetine: Assessment of the motor component. Journal of Affective Disorders, 43, 53-62.

Shallice, T. (1982). Specific impairments in planning. Philosophical transactions of the Royal Society of London series B Biological sciences, 298, 199-209.

Shallice, T. (1988). From neuropsychology to mental structure. Cambridge: Cambridge University Press.

Taylor, S.F. (1996). Cerebral blood flow activation and functional lesions in schizophrenia. Schizophrenia Research, 19, 129-140.

Thomassen, A.J.W.M., \& Tibosch, H.J.C.M. (1991). A quantitative model of graphic production. In: G.E. Stelmach \& J. Requin (Eds.), Tutorials in Motor Neuroscience (pp. 269-282). Dordrecht: Kluwer Academic Publishers.

Thomassen, A.J.W.M., Meulenbroek, R.G.J., \& Tibosch, H.J.C.M. (1991). Latencies and kinematics reflect graphic production rules. Human Movement Science, 10, 271-289.

Van Beilen, M., Withaar, F., Van Zomeren, A.H., \& Van den Bosch, R. (2002). Low IQ scores in schizophrenia: Primary or secondary deficit? Acta Neuropsychiatrica, 14, 106-110.

Van der Gaag, M. (1992). The results of cognitive training in schizophrenic patients. PhD. Dissertation. Groningen: University of Groningen.

Van der Gaag, M., Woonings, F.M.J., Van den Bosch, R.J., Appelo, M.T., Slooff, C.J., \& Louwerens, J.W. (1994). Cognitive training of schizophrenic patients: A behavioral approach based on experimental psychopathology. In: W.D. Spaulding (Ed.), Cognitive technology in psychiatric rehabilitation (pp. 139158). Nebraska: University of Nebraska Press.

Van der Gaag, M., Kern, R.S., Van den Bosch, R.J., \& Liberman, R.P. (2002). A controlled trial of cognitive remediation in schizophrenia. Schizophrenia Bulletin, 28, 167-176.

Van Hoof, J.J.M., Hulstijn, W., Van Mier, J.I.A.J., \& Pagen, M. (1993). Fine drawing and psychomotor retardation: Preliminary results. Journal of Affective Disorder, 29, 263-266.

Van Hoof, J.J.M. (2002). The abnormal development of drive and guidance mechanisms in the brain: The pathogenesis of schizophrenia. Acta Neuropsychiatrica, 14, 134-146.

Van Hoof, J.J.M. (2003). A motor hypothesis of the origin of schizophrenia (Letter to the editors). Schizophrenia Research, 62, 183-185.

Weaver, L.A., \& Brooks, G.W. (1964). The use of psychometric tests in predicting the potential of chronic schizophrenics. Journal of Neuropsychiatry, 5, 170-180.

Wechsler, D. (1956). Manual for the Wechsler Adult Intelligence Scale. Psychological corporation, New York. 


\section{4 | Chapter 8}

Weinberger, D.R., \& Berman, K.F. (1996). Prefrontal function in schizophrenia: Confounds and controversies. Philosophical transactions of the Royal Society of London series B Biological sciences, 351, 1495-1503.

Widlöcher, D., \& Hardy-Bayle, M.C. (1989). Cognition and control of action in psychopathology. European Bulletin of Cognitive Psychology, 9, 583-615.

Willingham, D.B. (1998). A neuropsychological theory of motor skill learning. Psychological Review, 105, 558-584.

Withaar, F., \& Arends, J. (2002). Cognitive rehabilitation in schizophrenia. In: W. Brouwer, E. van Zomeren, J. Berg, A. Bouma, \& E. de Haan (Eds.), Cognitive rehabilitation: A clinical neuropsychological approach (pp. 125-142). Amsterdam: Boom Publishers.

Wolkin, A., Sanfilipo, M., Wolf, A.P., Angrist, B., Brodie, J.D., \& Rotrosen, J. (1992). Negative symptoms and hypofrontality in chronic schizophrenia. Archives of General Psychiatry, 49, 959-965.

Wykes, T., Reeder, C., Corner, J., Williams, C., \& Everitt, B. (1999). The effects of neurocognitive remediation on executive processing in patients with schizophrenia. Schizophrenia Bulletin, 25, 291-307. 


\section{Summary}

Although schizophrenia is particularly known for the delusions and hallucinations, a qualified clinician may also recognize impairments associated with the illness in the behavior of patients and specifically in the way they perform motor actions. In clinical practice, there are numerous indications for the presence of psychomotor deficits, in which the term 'psychomotor' refers to a wide variety of activities requiring both cognitive and motor processes. Despite the fact that they have a large influence on the life of patients and the people surrounding them, psychomotor deficits are rarely a major topic of research in schizophrenia. The aim of the studies reported in the present thesis is to investigate the nature of these psychomotor deficits.

Chapter 1 describes the theoretical outline of the present thesis. Several views on the nature of psychomotor deficits are introduced. One theory states that schizophrenia can be particularly characterized by a disturbance in sensori(motor) or 'basic' psychomotor processes, which, in addition, hinders the execution and learning of more complex skills. The second theory claims that the major problem lies in the planning of complex self-motivated behavior (mostly indicated by the term 'executive dysfunction'). Motor deficits as well as executive deficits are, albeit separately, frequently observed in schizophrenia. Unclear, however, is whether and how these influence the performance of motor actions. The central questions in the present thesis are thus: to what extent are impairments in performing motor actions in schizophrenia (1) due to psychomotor slowing, and/or (2) affected by a more specific planning deficit. Another important question that we tried to answer was whether and how these impairments relate to clinical symptoms of schizophrenia.

Notwithstanding the clinical relevance of the classical neuropsychological approach, it seems less appropriate for the examination of psychomotor deficits in schizophrenia. The current neuropsychological tests were initially developed for the search for specific circumscribed brain lesions, whereas the disturbance in schizophrenia is far more diffuse. In addition, these tests do not measure one particular process; their scores are rather the result of the interplay of several cognitive and motor processes. This makes it difficult to discover which of these processes accounts most for the poor test performance. In the present thesis, it is tried to examine cognitive and motor processes underlying psychomotor deficits in schizophrenia from a cognitive neuropsychological approach. Following this approach, tasks are administered in which several experimental variables are manipulated, while the effects of these variables on task performance are examined. Graphic tasks, like drawing and writing, offer excellent possibilities for this cognitive neuropsychological viewpoint. When these tasks are employed on an electronic writing tablet, attached to a computer, they allow the analysis of several movement variables.

Psychomotor slowing is not unique for schizophrenia; it can also be observed in several other diseases, under which several psychiatric or neurological disorders. Particularly the question whether 'psychomotor poverty' in schizophrenia and 'psychomotor retardation' in depression are related or not, is not only of theoretical concern; it has also clinical relevance because schizophrenia 
can be accompanied by depressive symptoms. Chapter 2 describes a study in which this constitutes the central question. 'Psychomotor' speed is measured by means of a standard neuropsychological test, the Digit Symbol Test (DST). In this test, subjects are required to match digits and symbols as fast as possible according to a key consisting of nine digit-symbol pairs. Participants in the study were 20 patients with schizophrenia, 20 patients with a major depression, and 20 controls. Both patient groups had lower raw scores (number of correct digits filled in during 90 seconds) when compared to the control group, which indicated that they were slower in filling in the digits. A closer analysis of the task performance, however, demonstrated that the type of slowing differed between the patient groups. The depressed patients showed a general slowing, revealing itself in both a retarded matching time and writing time. The patients with schizophrenia only showed a slowing of the matching time. The results of this study suggested at the time a predominantly 'cognitive' slowing in schizophrenia, and a general, both 'cognitive' and 'motor' slowing, in depression. Later observations, however, suggested a more differentiated picture (Chapters 4 and 6). Actually, psychomotor slowing, in the more strict sense (expressing itself in a retarded writing time) appeared to be present in a subgroup of patients with schizophrenia. The question why this subgroup, and others not, showed psychomotor slowing deserves further exploration. In addition, it was a recurring result that particularly matching time (as a measure of cognitive slowing) varied with the type of setting in which the patients were staying. Moreover, in one of the studies (Chapter 6) it appeared to be significantly related with clinical features of cognitive disorganization. As the DST requires so many different cognitive functions (e.g., memory, attention and cognitive flexibility), we recommend not to include this test in the category of psychomotor tests.

Psychomotor skills are conceivably influenced by the use of antipsychotic medication. Chapter 3 describes the results of an exploratory study on the relationship between clinically observed 'bradykinesia' (slow moving) and performance on a number of fine motor tasks in a group of patients with schizophrenia or other psychotic disorder $(n=16)$. In general, bradykinesia arises as a side effect of antipsychotic medication, and can be determined by means of clinical rating scales. In the present study, we applied a recently developed rating scale, the 'Schedule for DrugInduced Movement Disorders' (SADIMoD). Another method that was applied was the measurement of the speed and precision of writing and drawing movements. The results showed that the ratings of bradykinesia correlated well with the performance on simple writing and drawing tasks, but not with that on a more complex figure-copying task. Performance on complex tasks will rather be influenced by potential cognitive deficits. The present study shows that the method of analyzing simple fine motor movements constitutes a reliable addition to the common clinical instruments for the assessment of side effects of medication.

Chapter 4 describes a study on possible planning disturbances in patients with schizophrenia. Participants were 19 patients with schizophrenia, and 19 control subjects matched on sex, age and education. Tasks that were applied were a line-copying task, a complex figure-copying task (both following a reaction paradigm) and the (previously mentioned) DST. The patients were, compared to the controls, about 33\% slower in their total performance time on all three tasks. This finding supported the notion that schizophrenia is associated with a general psychomotor slowing. The patients were particularly retarded in those variables that reflect cognitive processing, but 
appeared also to show problems with fine motor control. The patient group as a whole appeared to reinspect the stimuli significantly longer during the copying of the more complex and abstract patterns. This finding suggested that they were less able to plan their actions in advance. Initiation time prolonged due to increased figure complexity and unfamiliarity, but these latency increases were not significantly larger for the schizophrenia group as a whole, but only for a subgroup of patients with higher negative symptom scores. It was concluded that planning difficulties express themselves most clearly in patients with negative symptoms: they seem least able to plan the required movements before copying.

A major disadvantage of the complex figure-copying task (Chapter 4) for its use as a 'planning' task is that it requires a considerable portion of working memory capacity. To reduce the influence of a restricted working memory capacity, we also examined the planning of movements by using tasks in which the patterns to be copied consisted of not more than two or three lineelements (Chapters 5, 6 and 7). This type of tasks is based on the assumption that our graphic behavior is governed by certain 'implicit rules'. These rules specify preferences with respect to starting points, stroke directions and stroke sequences. In copying certain patterns (like the letter 'L'), these rules can be more or less easily applied. However, the copying of other patterns (like the same letter ' $\mathrm{L}$ ', but then mirrored around the $\mathrm{Y}$-axis) will confront people with conflicts between rules. It is assumed that for the drawing of 'non-conflicting' (NC) patterns a routine or automatic strategy suffices, whereas the production of 'conflicting' (C) patterns requires the planning of the most suitable movement sequence. Indeed, in healthy subjects, copying $\mathrm{C}$ patterns appear to result in a somewhat longer initiation time before starting to draw and in significantly longer hesitations above the paper ('pen-up time') during drawing when compared to their NC mirror images. In the study that is described in Chapter 5, the performance of a group of patients with schizophrenia ( $\mathrm{n}=$ $30)$ is compared with that of a control group $(n=20)$, and of a group of depressed patients $(n=30)$ with that of a second control group $(n=22)$. The hypothesis was that, because of a lack of planning, patients with schizophrenia would show fewer differences between the drawing of $\mathrm{NC}$ and $\mathrm{C}$ patterns, when compared to other groups. The results generally confirmed this hypothesis, in that differences between $\mathrm{NC}$ and $\mathrm{C}$ patterns were found in both control groups, and even more strongly in the depression group, but not in the schizophrenia group.

The patients with schizophrenia from Chapter 5 were, at the moment of testing, all selected to leave the psychiatric institute and to move to one of the residences outside. Later observations in a number of chronically hospitalized patients could not confirm these earlier findings. In these latter groups, clear differences in pen-up time (and sometimes initiation time) were found between the copying of $\mathrm{NC}$ and $\mathrm{C}$ patterns. Thus, their performance showed, paradoxically, more similarity with that of depressed patients. For a better understanding of these differences between patient groups, we decided to compare performance of the future outpatients $(\mathrm{n}=30)$ from Chapter 5 with that of a group of hospitalized patients $(n=34)$ directly. This is reported in Chapter 6. As the performance of the hospitalized patients paralleled that of the depressed patients more strongly, it could be expected that these hospitalized patients were showing more severe psychopathology and particularly marked depressive symptoms. The results, however, did not reveal clear differences in clinical symptoms between the both groups. The results rather showed that the experience of larger 
conflicts between rules was related with a stronger psychomotor slowing (as measured by means of a goal-directed movement task). In general, the findings of the study in Chapter 6 seem to demonstrate two types of planning problems in patients with schizophrenia. Patients that do not show psychomotor slowness appear to have a stronger preference for an automatic or stereotyped response pattern: they simply follow the most dominant rule and seem not to anticipate consequences of conflicting rules. Patients, on the other hand, that display psychomotor slowing appear to experience conflicts between rules to the same degree as depressed patients do.

The aim of the study described in Chapter 7 was to examine one type of inhibition, that is the ability to suppress an automatic response. It is known that this ability may affect the planning behavior of subjects. Performance of 20 patients with schizophrenia was compared with that of 20 control subjects. The patients appeared not to be slowed-down in copying simple lines or L-shaped patterns. However, when the position of the L-shaped patterns was varied in the working field, patients needed more initiation time than the controls. Closer inspection of this finding suggested that these prolonged initiation times could not be the result of problems with visual-spatial planning, but rather of a difficulty with deviating from a normal habitude to draw in the middle of the box. In another variant of this task, subjects were asked to start copying at a particular point (indicated by a small dot in the pattern itself). The patient group as a whole showed longer initiation times as a consequence of this imperative starting point. These initiation time increases were particularly found in patients with negative symptoms. Patients with cognitive disorganization, on the other hand, were reacting relatively fast in this forced task condition. This fast reaction pattern can be seen as an example of a stronger tendency to let their behavior determined by external stimuli. Considering the results of the present study, it can be concluded that the inability to suppress an automatic response presumably plays a crucial role in the planning difficulties of these patients.

In the final chapter, Chapter 8 , the main results are summarized. It was concluded that psychomotor slowing can be observed in a significant proportion of the patients with schizophrenia, but not in all. Those patients that do not show psychomotor slowness tend to use a more stereotyped way of performing motor actions. In addition, more specific planning deficits can be found in patients with schizophrenia, which, however, seem to vary with clinical symptoms like negative symptoms and cognitive disorganization. The most important conclusions are discussed in the context of a number of current theories of schizophrenia. Finally, the significance of the obtained results for clinical practice and future research is reviewed.

If we look beyond the findings of the studies reported in this thesis, it seems legitimate to state that psychomotor slowing cannot only be considered as resulting from problems in other neuropsychological domains (like executive function, attention or memory), but it in fact constitutes a problem by itself. Experimental research methods for the measurement of psychomotor deficits in schizophrenia are a useful completion to existing clinical instruments. The reason for this is that they reach beyond the description of symptoms. Moreover, the graphic tasks that are applied in the studies of the present thesis do not make a strong appeal to explicit processes, like in many other neuropsychological tests. Instead, they require more implicit adjustments in behavior, dependent on the context of the task. Most of our daily activities are not governed by explicit (and more or less 
'intellectual') rules, but rather by implicit choices. Therefore, the use of psychomotor tasks, in which one has to rely on more implicit choices between rules, might add to our understanding of why patients with schizophrenia encounter problems in daily life. 


\section{Samenvatting}

Hoewel schizofrenie vooral bekend is om de wanen en hallucinaties, zal de geoefende clinicus reeds in het gedrag van patiënten stoornissen waarnemen, specifiek in de motorische aspecten. In de klinische praktijk zijn voldoende aanwijzingen voor het bestaan van psychomotorische stoornissen bij schizofrenie, waarbij de term 'psychomotorisch' verwijst naar een zeer grote variëteit aan activiteiten die een beroep doen op allerlei cognitieve en motorische processen. Ondanks het feit dat deze psychomotorische stoornissen een grote invloed hebben op het dagelijkse leven van de patiënt en dat van zijn of haar omgeving, zijn ze relatief zelden het hoofdthema van onderzoek naar schizofrenie. Het doel van de studies die in dit proefschrift worden beschreven, is om de aard van deze psychomotorische stoornissen te onderzoeken.

In hoofdstuk 1 wordt de theoretische achtergrond van het proefschrift uiteengezet. Verschillende visies op de aard van psychomotorische stoornissen worden geïntroduceerd. In één theorie wordt gesteld dat er bij schizofrenie vooral sprake is van een verstoring van (sensori)motorische of 'basale' psychomotorische processen, wat vervolgens een belemmering vormt voor het leren en uitvoeren van meer complexe vaardigheden. Een andere theorie beweert dat het probleem primair ligt in het kunnen plannen en organiseren van complex zelfgemotiveerd gedrag (vaak aangeduid met de term 'executieve disfuncties'). Zowel motorische stoornissen als executieve disfuncties zijn bij schizofrenie, hoewel separaat, vaak onderzocht. Onduidelijk is echter óf en hóe deze van invloed zijn op het uitvoeren van motorische acties. De centrale vragen in dit proefschrift zijn dan ook: in hoeverre zijn problemen met het uitvoeren van motorische acties (1) te wijten aan een psychomotorische vertraging en/of (2) toe te schrijven aan een meer specifiek planningsprobleem. Een andere belangrijke vraag die wij probeerden te beantwoorden was in hoeverre psychomotorische vertraging en planningsproblemen verband houden met de klinische symptomen van schizofrenie.

Hoewel veel toegepast in de klinische praktijk, lijkt de klassieke neuropsychologische aanpak minder geschikt voor wetenschappelijk onderzoek naar psychomotorische stoornissen bij schizofrenie. De gebruikelijke neuropsychologische tests zijn immers oorspronkelijk ontwikkeld voor het opsporen van specifiek gelokaliseerde hersenbeschadigingen, terwijl de hersenstoornis bij schizofrenie veel meer diffuus lijkt. Zij meten verder niet één bepaald proces, maar een score op deze neuropsychologische tests komt tot stand door de samenwerking van meerdere cognitieve en motorische processen. Dit maakt het lastig na te gaan welke van deze processen het beste een testprestatie kan verklaren. In dit proefschrift wordt getracht via een cognitief-neuropsychologische aanpak inzicht te verkrijgen in meer specifieke processen die een rol spelen bij psychomotorische stoornissen bij mensen met schizofrenie. Hierbij worden taken uitgevoerd waarin verschillende experimentele variabelen worden gemanipuleerd, waarna wordt gekeken wat de invloed hiervan is op de prestaties. Grafische taken, zoals tekenen en schrijven, bieden uitstekende mogelijkheden voor deze cognitief-neuropsychologische benadering. Indien deze taken namelijk worden 
uitgevoerd op een elektronisch schrijftablet, dat gekoppeld is aan een computer, dan is het mogelijk verschillende bewegingsvariabelen te analyseren.

Psychomotorische vertraging komt niet alleen voor bij mensen met schizofrenie, maar kan zich ook bij andere ziekten voordoen, waaronder verschillende psychiatrische en neurologische aandoeningen. Met name de vraag of 'psychomotorische armoede' bij mensen met schizofrenie en 'psychomotorische retardatie' bij mensen met een depressie vergelijkbaar zijn of niet, is niet alleen theoretisch van belang, maar heeft ook duidelijk klinische relevantie. Immers, schizofrenie kan ook gepaard gaan met depressieve symptomen. In hoofdstuk 2 wordt een onderzoek beschreven waarin deze vraag centraal staat. 'Psychomotorische' snelheid is hierbij gemeten met behulp van een standaard neuropsychologische test, de Digit Symbol Test (DST). Bij deze test moeten proefpersonen zo snel mogelijk cijfers en symbolen combineren volgens een 'sleutel' die wordt gegeven. Aan het onderzoek namen 20 patiënten met schizofrenie deel, 20 patiënten met een depressie en 20 controle proefpersonen. Beide patiëntengroepen hadden gemiddeld een lagere ruwe score (aantal correct ingevulde cijfers gedurende 90 seconden) in vergelijking met de controlegroep. Dit betekent dat zij trager waren met het invullen van de cijfers. Een nadere analyse van de taakuitvoering liet echter zien dat de structuur van deze vertraging voor de twee groepen verschillend was. De patiënten met een depressie lieten een algehele vertraging zien die zich zowel uitte in een vertraagde zoektijd naar het betreffende cijfer als in een vertraagde schrijftijd. De patiënten met schizofrenie, daarentegen, lieten vooral een vertraging zien in de zoektijd. De resultaten van deze studie suggereerden indertijd een vooral 'cognitieve' vertraging bij patiënten met schizofrenie, en een algehele, cognitieve én motorische, vertraging bij patiënten met een depressie. Latere onderzoeksresultaten, echter, wezen in de richting van een meer gedifferentieerd beeld (hoofdstukken 4 en 6). Psychomotorische vertraging in meer strikte zin (tot uiting komend in een vertraagde schrijftijd) kwam wel degelijk ook voor bij een subgroep van patiënten met schizofrenie. Waarom deze subgroep deze psychomotorische vertraging vertoonde, verdient nog nader onderzoek. Verder viel op dat vooral de mate van cognitieve vertraging bij schizofrenie varieerde met het type setting waarin de patiënten verbleven. Daarnaast werd een significant verband gevonden met klinisch geobserveerde symptomen van cognitieve desorganisatie.

Aangezien de DST een beroep doet op zoveel verschillende cognitieve functies (o.a. geheugen, aandacht en cognitieve flexibiliteit) achten wij het raadzaam om deze test niet onder de noemer van 'psychomotorische' tests te scharen.

Psychomotorische vaardigheden worden mogelijkerwijs beïnvloed door het gebruik van antipsychotische medicatie. In hoofdstuk 3 worden de resultaten beschreven van een exploratief onderzoek naar de relatie tussen klinisch vastgestelde 'bradykinesie' (traag bewegen) en de prestaties op een aantal fijnmotorische taken bij een groep patiënten met schizofrenie en/of een psychotische stoornis $(\mathrm{n}=16)$. Bradykinesie treedt meestal op als een bijwerking van antipsychotische medicatie en kan worden vastgesteld met behulp van klinische observatieschalen. In het onderzoek werd gebruik gemaakt van een recent ontwikkeld beoordelingsinstrument, de 'Schedule for Drug-Induced Movement Disorders' (SADIMoD). Een andere methode, die werd toegepast voor het vaststellen van prestaties op fijnmotorische taken, was het kwantificeren van de snelheid en de precisie van schrijf- en tekenbewegingen. De resultaten lieten zien dat de 
bradykinesie-scores het beste correleerden met prestaties op eenvoudige schrijf- en tekentaken en niet met prestaties op complexere figuurkopieertaken. Laatstgenoemde taken zullen naar verwachting eerder worden beïnvloed door eventuele cognitieve tekorten. Dit onderzoek laat zien dat de methode waarmee eenvoudige fijnmotorische bewegingen worden geanalyseerd een goede aanvulling vormt op het gangbare klinische instrumentarium voor het meten van bijwerkingen van medicatie.

Hoofdstuk 4 beschrijft een onderzoek waarin gekeken is naar mogelijke planningsstoornissen bij mensen met schizofrenie. Aan het onderzoek namen 19 patiënten met schizofrenie deel en 19 (op geslacht, leeftijd en opleiding gematchte) controle proefpersonen. De taken die zijn afgenomen waren een lijnkopieertaak, een complexere figuurkopieertaak (beide volgens een reactietijdparadigma) en de (eerdergenoemde) DST. De patiënten met schizofrenie waren, vergeleken met de controles, in de totale uitvoeringstijd ongeveer $33 \%$ trager op alle drie de taken. Dit is een ondersteuning van de opvatting dat schizofrenie gepaard gaat met een algehele psychomotorische vertraging. De patiënten waren vooral vertraagd in bewegingsvariabelen die cognitieve processen reflecteren, maar gaven daarnaast ook blijk van problemen met fijnmotorische controle. De totale patiëntengroep bleek tijdens het natekenen van de meer complexe en abstracte figuren significant langer gebruik te maken van de mogelijkheid om deze figuren te herinspecteren, hetgeen er op duidt dat zij hun acties van tevoren minder goed konden plannen. De reactietijd nam toe als gevolg van een toename in complexiteit en in onbekendheid van de figuren. Dit bleek echter niet significant te zijn bij de patiëntengroep als geheel, maar alleen bij een subgroep van patiënten met hogere scores op negatieve symptomen. Geconcludeerd werd dat planningsproblemen het duidelijkst tot uiting komen bij patiënten met negatieve symptomen: zij lijken het minst in staat om de vereiste bewegingen van tevoren te plannen.

Een belangrijk nadeel van de complexere figuurkopieertaak, die in het onderzoek van hoofdstuk 4 werd gebruikt als een 'planningstaak', is dat er een groot beroep wordt gedaan op wat wordt aangeduid als het werkgeheugen. Om de invloed van een beperkt werkgeheugen zoveel mogelijk te reduceren, is het plannen van bewegingen ook onderzocht met behulp van taken waarin de figuurtjes uit niet meer dan twee of drie lijnsegmenten bestonden (hoofdstukken 5, 6 en 7). Dit type taak is gebaseerd op de assumptie dat ons grafische gedrag onder invloed staat van bepaalde 'impliciete regels'. Deze hebben betrekking op voorkeuren voor startposities, tekenrichtingen en tekenvolgordes. Bij het tekenen van bepaalde figuurtjes (zoals de letter 'L') kunnen deze regels zonder problemen worden gevolgd. Bij andere figuurtjes, daarentegen, (bijvoorbeeld dezelfde letter 'L', maar dan gespiegeld om de Y-as) zal men conflicten tussen de regels gaan ervaren. Aangenomen wordt dat het tekenen van een 'niet-conflicterende' (NC) figuur op een automatische wijze verloopt, terwijl bij het tekenen van een 'conflicterende' (C) figuur een geschikte bewegingsvolgorde moet worden gepland. Deze C figuurtjes blijken bij gezonde proefpersonen ook enigszins langere initiatietijden en tijdens het tekenen bovenal langere aarzelingen boven het papier ('pen-optil-tijd') op te leveren, vergeleken met de NC spiegelbeelden. In het onderzoek dat beschreven staat in hoofdstuk 5 werden de prestaties vergeleken van een groep patiënten met schizofrenie $(\mathrm{n}=30)$ met een controlegroep $(\mathrm{n}=20)$ en van een groep patiënten met een depressie $(n=30)$ met een tweede controlegroep $(n=22)$. De hypothese in dit onderzoek was dat patiënten 
met schizofrenie, vergeleken met de andere groepen, door een gebrek aan planning minder verschillen zouden vertonen tussen het natekenen van $\mathrm{NC}$ en $\mathrm{C}$ figuren. De resultaten bevestigden voor een groot deel deze hypothese, in die zin dat er verschillen tussen de $\mathrm{NC}$ en $\mathrm{C}$ figuren gevonden werden in de beide controlegroepen, zelfs sterker in de groep patiënten met een depressie, maar níet in de groep patiënten met schizofrenie.

De patiënten met schizofrenie uit hoofdstuk 5 waren, op het moment van het onderzoek, allen geselecteerd om het verblijf binnen een psychiatrische instelling te verruilen voor een beschermende of begeleide woonvorm. Latere observaties bij een aantal langdurig gehospitaliseerde groepen patiënten konden de eerdere resultaten niet bevestigen. In laatstgenoemde groepen werd een duidelijk verschil in pen-optil-tijd (en soms initiatietijd) gevonden bij het natekenen van de NC en $\mathrm{C}$ figuurtjes. Daarmee vertoonden hun prestaties, paradoxaal genoeg, meer het beeld van de patiënten met een depressie. Om inzicht te krijgen in deze verschillen tussen patiëntengroepen werd besloten om de prestaties van de (toekomstige) ambulant verblijvende patiënten $(\mathrm{n}=30)$ uit hoofdstuk 5 rechtstreeks te vergelijken met die van een groep gehospitaliseerde patiënten $(n=34)$. Hiervan wordt verslag gedaan in hoofdstuk 6. Aangezien gebleken was dat de prestaties van de gehospitaliseerde patiënten met schizofrenie meer overeenkomsten vertoonden met de prestaties van patiënten met een depressie, kon verwacht worden dat deze gehospitaliseerde patiënten meer psychopathologie vertoonden en met name meer depressieve symptomen. Echter, de resultaten lieten geen duidelijke verschillen zien in klinische symptomen tussen de beide groepen. Wel bleek dat het ervaren van grotere conflicten tussen regels gerelateerd was aan een sterkere psychomotorische vertraging (gemeten met behulp van een doelgerichte bewegingstaak). In zijn algemeenheid lieten de resultaten van het onderzoek uit hoofdstuk 6 twee typen planningsproblemen zien bij patiënten met schizofrenie. Patiënten die géén psychomotorische vertraging vertonen lijken een sterke voorkeur te hebben voor een automatische of stereotiepe strategie: zij volgen gewoon de meest dominante regel en lijken niet te anticiperen op conflicten tussen regels. Patiënten die daarentegen wèl psychomotorische vertraging vertonen lijken de conflicten tussen regels in dezelfde mate te ervaren als patiënten met een depressie.

In het onderzoek van hoofdstuk 7 is gekeken naar één van de vormen van inhibitie, namelijk het vermogen om een automatische respons te onderdrukken. Bekend is dat dit van invloed kan zijn op het planningsgedrag van personen. De prestaties van 20 patiënten met schizofrenie werden vergeleken met die van 20 controle proefpersonen. De patiënten bleken niet trager te zijn in het natekenen van eenvoudige lijntjes of L-vormige figuurtjes. Echter, wanneer de positie van de Lfiguurtjes werd gevarieerd in het tekengebied, hadden de patiënten meer initiatietijd nodig dan de controlegroep. Nadere analyses lieten zien dat deze langere initiatietijden niet het gevolg konden zijn van problemen met de visueel-spatiële planning, maar eerder van het moeten afwijken van een gewoonte om het figuurtje in het midden te tekenen. In een andere taakvariant werd aan de proefpersonen gevraagd om bij een bepaald punt (aangegeven met behulp van een klein 'startbolletje') te beginnen met het natekenen. De patiëntengroep in zijn geheel liet hierbij een langere initiatietijd zien. Deze langere initiatietijd werd echter vooral gevonden bij patiënten met negatieve symptomen. Patiënten met cognitieve desorganisatie, daarentegen, reageerden relatief snel op dit gedwongen startpunt. Dit kan gezien worden als een gevolg van de neiging om het 
gedrag sterker te laten bepalen door externe stimuli of prikkels. Op basis van de resultaten van dit onderzoek kan worden geconcludeerd dat het onvermogen om een automatische respons te onderdrukken een cruciale rol speelt bij de planningsproblemen van deze patiënten.

In het afsluitende hoofdstuk 8 worden de voornaamste resultaten samengevat. Geconcludeerd werd dat psychomotorische vertraging bij een substantieel deel van de patiënten met schizofrenie voorkomt, maar niet bij allemaal. Patiënten die niet psychomotorisch vertraagd waren, lieten in het uitvoeren van motorische acties een meer stereotiep patroon zien. Voorts werden meer specifieke planningsproblemen gevonden bij patiënten met schizofrenie, die echter bleken te variëren met klinische symptomen zoals negatieve symptomen en cognitieve desorganisatie. De belangrijkste conclusies zijn tegen het licht gehouden van een aantal actuele theorieën over schizofrenie. Tot slot is ingegaan op de betekenis van de onderzoeksresultaten voor de klinische praktijk en voor toekomstig onderzoek.

Niet alleen op basis van de bevindingen in dit proefschrift lijkt het legitiem om te stellen dat psychomotorische vertraging niet slechts gezien moet worden als het resultaat van problemen in andere neuropsychologische domeinen (zoals executieve functies, aandacht of geheugen) maar dat het een probleemgebied op zich vormt. Experimentele onderzoeksmethoden voor het meten van psychomotorische stoornissen bij schizofrenie kunnen een belangrijke aanvulling zijn op het gangbare klinische instrumentarium. Deze gaan immers verder dan de beschrijving van symptomen. Bovendien doen de grafische taken zoals toegepast in de studies van dit proefschrift niet zozeer een beroep op allerlei expliciete processen, zoals bij veel andere neuropsychologische testen. Ze vragen eerder impliciete aanpassingen van het gedrag, afhankelijk van de context van de taak. Ook bij de meeste dagelijkse activiteiten worden niet allerlei expliciete (en min of meer 'intellectuele') regels gehanteerd maar worden eerder impliciete keuzes gemaakt. Daarom zal de toepassing van psychomotorische taken waarbij men min of meer impliciete keuzes tussen regels moet maken, bijdragen aan een beter begrip van het feit dat patiënten met schizofrenie problemen ervaren in hun dagelijkse leven. 


\section{Curriculum Vitae}

Bea Jogems-Kosterman werd geboren op 30 december 1969 te Wijk bij Duurstede. Na het behalen van het VWO diploma an het St. Bonifatiuscollege te Utrecht in 1988, volgde zij de studie bewegingswetenschappen aan de Vrije Universiteit te Amsterdam. Het laatste jaar van de opleiding stond in het teken van een onderzoeksstage bij de Afdeling Research \& Ontwikkeling van de St. Maartenskliniek (hoofd: Prof. dr. Th. Mulder) te Nijmegen. In 1993 studeerde zij af in de bewegingswetenschappen met 'psychologie met betrekking tot het menselijk bewegen' als hoofdrichting. In datzelfde jaar begon zij als wetenschappelijk medewerker bij het IPZ Oost Brabant op de locatie Coudewater te Rosmalen (thans onderdeel uitmakend van de GGZ Oost Brabant). In de periode 1993-1994 heeft zij daarnaast als onderzoeksmedewerker gewerkt aan een door NWO gesubsidieerd project op de afdeling psychiatrie van het St. Radboud Ziekenhuis te Nijmegen (Prof. dr. F.G. Zitman). Tot op heden is zij als wetenschappelijk medewerker bij de GGZ Oost Brabant, verbonden aan de A-opleiding van psychiaters (opleider: Dr. J.J.M. van Hoof), verantwoordelijk voor en betrokken bij verschillende onderzoeksprojecten, onder andere de projecten waarop dit proefschrift is gebaseerd. Veel van deze onderzoeken zijn (en worden) uitgevoerd in nauwe samenwerking met het Nijmeegs Instituut voor Cognitie en Informatie (NICI; Prof. dr. W. Hulstijn). In totaal heeft zij 14 doctoraal-studenten begeleid tijdens de uitvoering van hun afstudeeronderzoek. Daarnaast begeleidde zij verschillende arts-assistenten bij het verrichten van (wetenschappelijk) onderzoek in het kader van hun opleiding tot psychiater. Sinds 1996 is zij, naast haar functie als wetenschappelijk medewerker, ook werkzaam als stafmedewerker bij bureau zorgontwikkeling (hoofd: Drs. L.J. Lunshof; sinds kort interim: Drs. A.A.M. van den Berg) van de GGZ Oost Brabant. Tot op heden fungeert zij met name ook als katalysator op het terrein van zorggebonden wetenschappelijk onderzoek binnen de instelling. Dit blijkt onder andere uit haar lidmaatschap, namens de GGZ Oost Brabant, van de METIGG (Stichting Medisch-Ethische Toetsingscommissie Instellingen Geestelijke Gezondheidszorg) en uit haar functie als secretaris van de interne wetenschappelijke commissie. 


\section{Publications}

\section{Contributions to refereed articles and reviewed manuscripts}

Kosterman, B.J.M., Westzaan P.S.H., \& Van Wieringen, P.C.W. (1994). Developmental trends of fine motor performance in primary school children: A kinematic analysis. In: C. Faure, P. Keuss, G. Lorette \& A. Vinter (Eds.), Advances in handwriting and drawing: A multidisciplinary approach (pp 247-258). Paris: Europia.

De Jong, W.P., Hulstijn, W., Jogems-Kosterman, B.J.M., \& Smits-Engelsman, B.C.M. (1996). OASIS software and its application in experimental handwriting research. In: M.L. Simner, C.G. Leedman \& A.J.W.M. Thomassen (Eds.), Handwriting and Drawing Research: Basic and Applied Issues (pp. 429-440). Amsterdam: IOS Press.

Van Hoof, J.J.M., Sabbe, B.G.C., Hulstijn, W., Van Mier, H., \& Jogems-Kosterman, B.J.M. (1996). Het kopiëren van figuren en psychomotorische vertraging bij depressie. Tijdschrift voor Psychiatrie, 38, 520-531.

Loonen, A.J.M., Doorschot, C.H., Jogems-Kosterman, B.J.M., Van Hoof, J.J.M., Hulstijn, W. \& Zitman, F.G. (1997). Nieuw instrumentarium voor het meten van bijwerkingen. Cobo-bulletin, $1,31-40$.

Jogems-Kosterman, B.J.M., Loonen, A.J.M., Van Hoof, J.J.M., Zitman F.G., \& Hulstijn, W. (1998). Antipsychotica, bewegingsstoornissen en fijne motoriek: Nieuw instrumentarium voor het meten van bradykinesie en tremoren. Acta Neuropsychiatrica, 10, 93-99.

Van Hoof, J.J.M., Jogems-Kosterman, B.J.M., Sabbe, B.G.C., Zitman, F.G., \& Hulstijn, W. (1998). Differentation of cognitive and motor slowing in the Digit Symbol Test (DST): Differences between depression and schizophrenia. Journal of Psychiatric Research, 32, 99-103.

Jogems-Kosterman, B.J.M., Hulstijn, W., Van Hoof, J.J.M., \& Thomassen, A.J.W.M. (1999). Is the planning of action in schizophrenic patients more impaired than in depressed patients? The effects of conflicting graphic production rules. In: G. Leedham, M. Leung, V. Sagar \& X. Xuhong (Eds.), Proceedings of the 9th Biennial Conference of the International Graphonomics Society (pp. 225-229). Singapore: NTU.

Fakkers, G.F.M., Jogems-Kosterman, B.J.M., Loonen, A.J.M., \& Van Hoof, J.J.M. (2000). Neurocognitief onderzoek als voorspeller van functioneren bij schizofrenie. Cobo-bulletin, 1, 27-34.

Loonen, A.J.M., Doorschot, C.H., Van Hemert, D.A., Oostelbos, M.C.J.M., Sijben, A.E.S., \& the MASEAS Team ${ }^{1}$ (2000). The Schedule for the Assessment of Drug-Induced Movement Disorders (SADIMoD): Test-retest reliability and concurrent validity. International Journal of Neuropsychopharmacology, 3, 285 - 296.

Hulstijn, W., Jogems-Kosterman, B.J.M., Wezenberg, E., \& Sabbe, B.G.C. (2001). An evaluation of the use of figure-copying tasks in studies of planning deficits in schizophrenia. In: R.G.J.

\footnotetext{
${ }^{1}$ The MASEAS Team consisted of the examiners and/or raters and the camera-operators making the video-recordings of six participating centres, including B. Jogems (examiner and rater).
} 
Meulenbroek \& B. Steenbergen (Eds.), Proceedings of the tenth biennial conference of the International Graphonomics Society (pp. 46-51). Nijmegen: IGS.

Jogems-Kosterman, B.J.M., Zitman, F.G., Van Hoof, J.J.M., \& Hulstijn, W. (2001). Psychomotor slowing and planning deficits in schizophrenia. Schizophrenia Research, 48, 317-333.

Loonen, A.J.M., Doorschot, C.H., Van Hemert, D.A., Oostelbos, M.C.J.M., Sijben, A.E.S., \& the MASEAS Team ${ }^{1}$ (2001). The Schedule for the Assessment of Drug-Induced Movement Disorders (SADIMoD): Inter-rater reliability and construct validity. International Journal of Neuropsychopharmacology, 4, 347-360.

Hulstijn, W., Jogems-Kosterman, B.J.M., Van Hoof, J.J.M., \& Sabbe, B.G.C. (2002). Planningsstoornissen bij schizofrenie. Nieuwe grafische onderzoeksmethoden. Tijdschrift voor Psychiatrie, 44, 739-745.

Jogems-Kosterman, B.J.M., Hulstijn, W., Wezenberg, E., \& Van Hoof, J.J.M. (accepted in a shortened and revised version). Movement planning deficits in schizophrenia: Failure to inhibit automatic response tendencies. Cognitive Neuropsychiatry.

\section{Contributions to published abstracts}

Jogems-Kosterman, B.J.M., Zitman, F.G., Van Hoof, J.J.M., Loonen, A.J.M., De Jong, W.P., \& Hulstijn, W. (1997). Tremor in schizophrenic patients treated with antipsychotics. The Journal of European College of Neuropharmacology, 7, S204-205.

Jogems-Kosterman, B.J.M., Zitman, F.G., Van Hoof, J.J.M., Loonen A.J.M., \& Hulstijn, W. (1998). Psychomotor slowing in schizophrenic patients: Movement variables and cognitive manipulations. Schizophrenia Research, 29, 58.

Van Dijke, I., Jogems-Kosterman, B.J.M., \& Van Hoof, J.J.M. (1998). The performance of a psychomotor planning task with schizophrenia. Schizophrenia Research, 29, 45.

Van Hoof, J.J.M., Jogems-Kosterman, B.J.M., Sabbe, B.G.C., Zitman, F.G., \& Hulstijn, W. (1998). Differentation of cognitive and motor slowing in the Digit Symbol Test (DST): Differences between depression and schizophrenia. Schizophrenia Research, 29, 57.

Jogems-Kosterman, B.J.M., Hulstijn, W., Van Hoof, J.J.M., \& Thomassen, A.J.W.M. (1999). Is the planning of action in schizophrenic patients more impaired than in depressed patients? The effects of conflicting graphic production rules. Poster presented at the $21^{\text {st }}$ International Summer School of Brain Research (Topic: Cognition, emotion and automatic responses). Amsterdam: Nederlands Institute for Brain Research.

Jogems-Kosterman, B.J.M., Hulstijn, W., Van Hoof, J.J.M., \& Thomassen, A.J.W.M. (2000). Studying planning in graphic production: Evidence for more stereotyped behaviour in schizophrenic patients compared to depressed patients and controls. Schizophrenia Research, $41,286$.

Oliemeulen, E.A.P., Van Hoof, J.J.M., Jogems-Kosterman, B.J.M., Hulstijn, W., \& Tuynman-Qua, H.G. (2000). Is olanzapine a substitute for clozapine? The effects on psychomotor performance. Schizophrenia Research, 41, 187. 
Fakkers, G.P.F.M., Jogems-Kosterman, B.J.M., Loonen, A.J.M., Van Hoof, J.J.M., \& Hulstijn, W. (2002). Changes in clinical functioning and neurocognitive performances in patients with schizophrenia during the first years of deinstitutionalization. Schizophrenia Research, 53, 127.

Jogems-Kosterman, B.J.M., Hulstijn, W., Wezenberg, E. \& Van Hoof, J.J.M. (2002). Studying planning in patients with schizophrenia by means of simple figure-copying tasks. Schizophrenia Research, 53, 135. 
162 


\section{Dankwoord}

De totstandkoming van dit proefschrift was onmogelijk geweest zonder de betrokkenheid en hulp van velen. Allereerst wil ik de patiënten bedanken die veelal belangeloos aan de onderzoeken hebben deelgenomen. In totaal hebben 140 patiënten met schizofrenie en 50 patiënten met een depressie hun medewerking verleend; voor het merendeel afkomstig van de GGZ Oost Brabant, maar ook deels van de GGz Nijmegen en het UMC St. Radboud te Nijmegen.

Mijn belangrijkste compagnon tijdens het schrijven van dit proefschrift en de daaraan voorafgaande onderzoekingen, was Wouter Hulstijn (eerste promotor). Wouter, bedankt voor je tomeloze ijver en je geduld bij het bespreken en interpreteren van de vele onderzoeksresultaten. Ik heb enorm veel van je kunnen leren wat betreft het helder verwoorden van gedachten en het vasthouden van de rode draad in artikelen en in dit proefschrift. Jouw kritische houding heeft mij maximaal gestimuleerd om tot een goed eindproduct te komen.

Mijn trouwe compagnon bij de GGZ Oost Brabant was Jacques van Hoof (copromotor). Jacques, jouw enthousiasme voor dit onderzoek en het belang dat je daaraan hechtte - niet alleen voor de klinische praktijk maar ook voor de theorievorming - zijn voor mij een belangrijke 'motor' geweest om dit werk te voltooien. Tevens heb je mij weten te prikkelen door je vaak frisse en vernieuwende kijk op het vakgebied psychiatrie, in het bijzonder je zienswijze op het ontstaan van psychopathologie. Jacques, onze intensieve samenwerking in de afgelopen tien jaar heeft mij hoe dan ook gevormd: ik ben er sterker door geworden.

Graag wil ik Ar Thomassen (tweede promotor) bedanken. Ar, ik ben je zeer erkentelijk voor je wijze raadgevingen tijdens het traject, je correcties van de teksten en de speciale aandacht die je had voor mijn persoonlijke situatie: de niet altijd gemakkelijke combinatie van een werkse carrière en het moederschap.

Dit promotietraject heb ik kunnen doorlopen dankzij de mogelijkheid die mij vanuit mijn werkplek bij de GGZ Oost Brabant is geboden. Hiervoor ben ik niet alleen mijn voormalige leidinggevenden Jacques van Hoof en Bert Lunshof, maar ook de Raad van Bestuur dankbaar.

Mijn vaste maatjes binnen de GGZ Oost Brabant waren Lisette Oliemeulen en Geert Fakkers (paranimfen). Lisette, bedankt dat ik jouw kamergenoot mocht zijn en dat wij veel activiteiten samen hebben kunnen doen. Daarbij heb ik bovenal genoten van jouw optimistische kijk op de dingen en de onbevangenheid waarmee jij in het leven staat. Geert, ik waardeer je authentieke houding in je contact met mensen, in het bijzonder met de kwetsbare mensen binnen de geestelijke gezondheidszorg. Jouw vermogen om te relativeren hielp mij door moeilijke momenten heen.

Anton Loonen ben ik dankbaar voor zijn betrokkenheid bij het onderzoek vanaf 'het eerste uur'. Anton, dank voor je vaste bereidheid mij te adviseren, niet alleen op methodologisch vlak maar ook voor wat betreft het maken van meer strategische keuzes in de verschillende onderzoekstrajecten.

Frans Zitman, dank voor de prettige samenwerking op de afdeling psychiatrie van het UMC St. Radboud. 
In de loop van de jaren hebben verschillende studenten hun stage/scriptie-onderzoek bij mij uitgevoerd. Van hen wil ik in het bijzonder noemen: Yvonne Maas, Ingeborg van Dijke, Esther Wentink en Elke Wezenberg. Hun bijdrage is ook verwerkt in dit proefschrift.

Peter de Jong, achter de schermen was jij onmisbaar. Jij zorgde er voor dat de programmatuur die ik nodig had voor het onderzoek altijd tiptop in orde was.

Mijn collega's van bureau zorgontwikkeling wil ik bedanken voor de fijne werkplek. Een aantal van hen wil ik in het bijzonder noemen. Bert Lunshof, bedankt voor je coaching in de afgelopen acht jaar. Ik heb van je geleerd dat een gestructureerde aanpak van het werk veel voordelen biedt. Ton van den Berg, bedankt voor je enorme betrokkenheid en steun in de laatste 'zware' fase van het promotietraject en voor je taalkundige correcties van de Nederlandse teksten. Janine de Jong, bedankt voor je luisterend oor.

Hanneke Meulenbroek wil ik bedanken voor haar correcties van het Engels van verschillende artikelen.

Dan de mensen die heel dicht bij mij staan. Mijn ouders ben ik in het bijzonder dankbaar voor de warmte en de liefde waarin ik mocht opgroeien en voor hun steun en betrokkenheid tot op de dag van vandaag. Ineke, je bent vast en zeker trots op je zusje; dat ben ik ook op jou. Riet, Frank's moeder, bedankt voor je hulp en inzet bij het reilen en zeilen van ons gezin. Frank, lief en leed kan ik met je delen; bedankt voor de overgave waarmee je onze kinderen opvoedt, je steun en de interesse die wij samen delen voor de psyche van de mens. Anne-Ruth en Bernice, hoewel ik soms stiekem wel eens hoop dat jullie ook wat meer psychomotorisch geremd zouden zijn...., geniet ik elke dag van jullie. Mijn hemelse Vader ben ik dankbaar voor de kracht en de inspiratie die ik van Hem ontvang: dag aan dag draagt Hij mij. 